\title{
Review of Geophysical Characterization Methods Used at the Hanford Site
}

\author{
G. V. Last \\ D. G. Horton
}

March 2000

Prepared for the U.S. Department of Energy under Contract DE-AC06-76RLO 1830 


\title{
DISCLAIMER
}

This report was prepared as an account of work sponsored by an agency of the United States Government. Neither the United States Government nor any agency thereof, nor Battelle Memorial Institute, nor any of their employees, makes any warranty, express or implied, or assumes any legal liability or responsibility for the accuracy, completeness, or usefulness of any information, apparatus, product, or process disclosed, or represents that its use would not infringe privately owned rights. Reference herein to any specific commercial product, process, or service by trade name, trademark, manufacturer, or otherwise does not necessarily constitute or imply its endorsement, recommendation, or favoring by the United States Government or any agency thereof, or Battelle Memorial Institute. The views and opinions of authors expressed herein do not necessarily state or reflect those of the United States Government or any agency thereof.

\author{
PACIFIC NORTHWEST NATIONAL LABORATORY \\ operated by \\ BATTELLE \\ for the \\ UNITED STATES DEPARTMENT OF ENERGY \\ under Contract DE-ACO6-76RLO 1830
}

Printed in the United States of America

Available to DOE and DOE contractors from the

Office of Scientific and Technical Information, P.O. Box 62, Oak Ridge, TN 37831;

prices available from $(615) 576-8401$.

Available to the public from the National Technical Information Service, U.S. Department of Commerce, 5285 Port Royal Rd., Springfield, VA 22161

T) This document was printed on recycled paper. 
PNNL-13149

\title{
Review of Geophysical Characterization Methods Used at the Hanford Site
}

\author{
G. V. Last \\ D. G. Horton
}

March 2000

Prepared for

the U.S. Department of Energy

under Contract DE-AC06-76RLO 1830

Pacific Northwest National Laboratory

Richland, Washington 99352 


\begin{abstract}
Geophysical methods have been used for characterization of hydrogeologic conditions and/or contaminant distributions at the Hanford Site since the mid- to late-1940s. A review of these geophysical methods is presented in two parts: 1) shallow surface-based geophysical methods and 2) borehole geophysical-logging methods.

Virtually all types of surface-based geophysical methods have been tested, including groundpenetrating radar (GPR), numerous electromagnetic, magnetic, seismic, and gravity methods. To date, over 250 geophysical surveys have been conducted in portions of every "Area" of the Hanford Site. The most widely used geophysical methods are GPR, frequency-domain electromagnetics (i.e., Geonics EM-31), and metal detectors.

The geologic formations that make up the vadose zone are unconsolidated; thus, virtually every borehole is cased with schedule 40 steel pipe. The casing, and in later years a grout annular seal, have been the biggest factors in determining the types of logging methods used. Traditionally, gross gamma ray and neutron moisture probes were the two most commonly used downhole tools. Recently, spectral gamma-ray logging has replaced gross gamma-ray logging for most applications. Many other techniques - including prompt fission neutron logging and neutron-gamma logging for specific elemental analyses - have been tested and/or used for contaminant and lithologic characterization in the subsurface.
\end{abstract}




\section{Acknowledgments}

The authors wish to express their gratitude to Thomas H. Mitchell and Kevin A. Bergstrom of CH2M Hill Hanford, Inc., and Gerald A. Sandness at the Pacific Northwest National Laboratory, for opening up their data files for our review, and for the advice during the course of this work. We would also like to thank Samatha D. Tippery for her support in the collection and formatting of bibliographic reference materials. The authors thank the project managers Glendon W. Gee and Anderson L. Ward for providing guidance and support throughout this effort. The authors also thank Christopher J. Murray and Gerald A. Sandness for their review of all or portions of this document and their valuable comments/additions. The authors also wish to express their gratitude to the Science and Technology endeavor of the Groundwater

and Vadose Zone Project team members. A note of appreciation also goes to Jo Lynn Draper and Barbara Wilson, the editors, and the text processor, Lila Andor. 


\section{Contents}

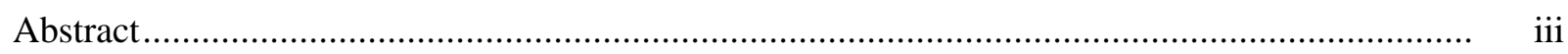

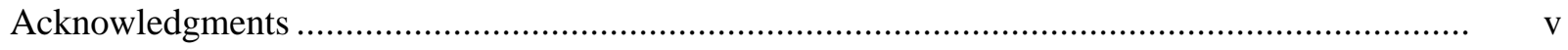

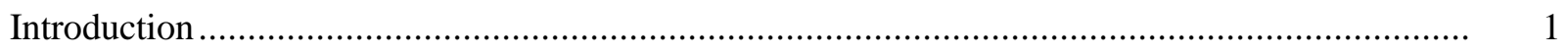

Shallow Surface-Based Geophysical Methods ................................................................................ 1

Ground-Penetrating Radar …………………………………………………………...

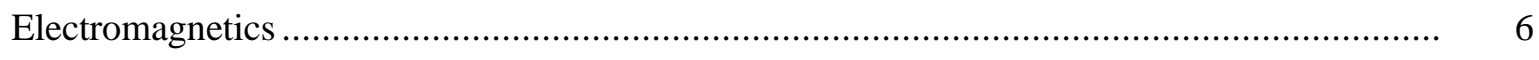

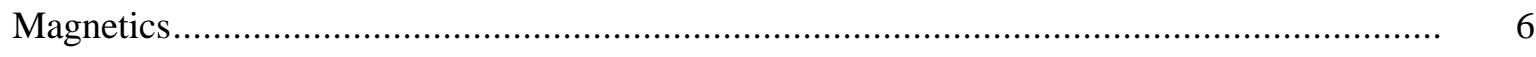

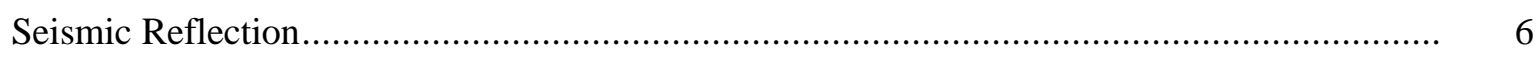

Borehole Geophysical Logging Methods............................................................................... 6

Gross Gamma-Ray Logging ……………………………………………………...

Spectral Gamma-Ray Logging ....................................................................................... 7

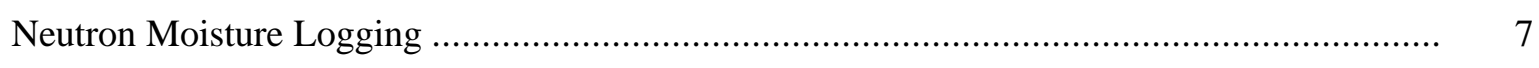

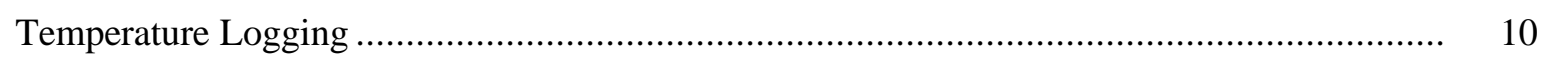

Other Geophysical Borehole Logging ......................................................................... 10

Appendix A - Catalog of Shallow Surface Geophysical Surveys Arranged by Site ....................... A.1

Appendix B - Bibliography with Abstracts Regarding Shallow Surface Geophysical Surveys at the Hanford Site.................................................................................. B. B.

Appendix C - Bibliography with Abstracts Regarding Borehole Geophysics at the Hanford Site

C. 1 


\section{Figure}

1 Map of the Hanford Site's "Areas" .................................................................................. 2

\section{Tables}

1 Summary of Shallow Surface-Based Geophysics at the Hanford Site.................................. 3

2 Summary of Borehole Geophysical Logging Methods Used at Hanford ............................. 8 


\section{Introduction}

This paper presents a review of geophysical methods used at Hanford in two parts, 1) shallow surface-based geophysical methods and 2) borehole geophysical methods. This review was not intended to be "all encompassing," but should represent the vast majority (>90\% complete) of geophysical work conducted onsite and aimed at hazardous waste investigations in the vadose zone and/or uppermost groundwater aquifer(s). This review did not cover geophysical methods aimed at large-scale geologic structures or seismicity and, in particular, did not include those efforts conducted in support of the Basalt Waste Isolation Program. This review focused primarily on the more recent efforts.

Brief summaries of the geophysical methods used at the Hanford Site follow. Included in the appendices are:

- a catalog of the surface-based geophysical surveys conducted to date (Appendix A)

- a bibliography of Hanford-specific surface-based geophysics (Appendix B)

- a bibliography of Hanford-specific borehole logging geophysics (Appendix C).

The reference numbers used throughout this report correspond to appendix and numerical listing for reference in the corresponding bibliography.

\section{Shallow Surface-Based Geophysical Methods}

Geophysical methods have been used for the determination of geologic conditions at the Hanford Site since 1955 (B-108). The use of geophysics to support hazardous waste site investigations at the Hanford Site were initiated by 1975 (B-103 and -104]). Virtually all types of geophysical methods have been tested including ground-penetrating radar (GPR), numerous electromagnetic, magnetic, seismic, and gravity methods. To date, more than 250 geophysical surveys have been conducted in portions of every "Area" of the Hanford Site (Figure 1). Appendix A is a catalog of these geophysical surveys, arranged by Area and Site. Table 1 provides a synopsis of this catalog, by describing the methods used, where and when they were used, the objectives of the surveys, and a list of available references. The most widely used geophysical methods are GPR and electromagnetic induction (including metal detectors). 


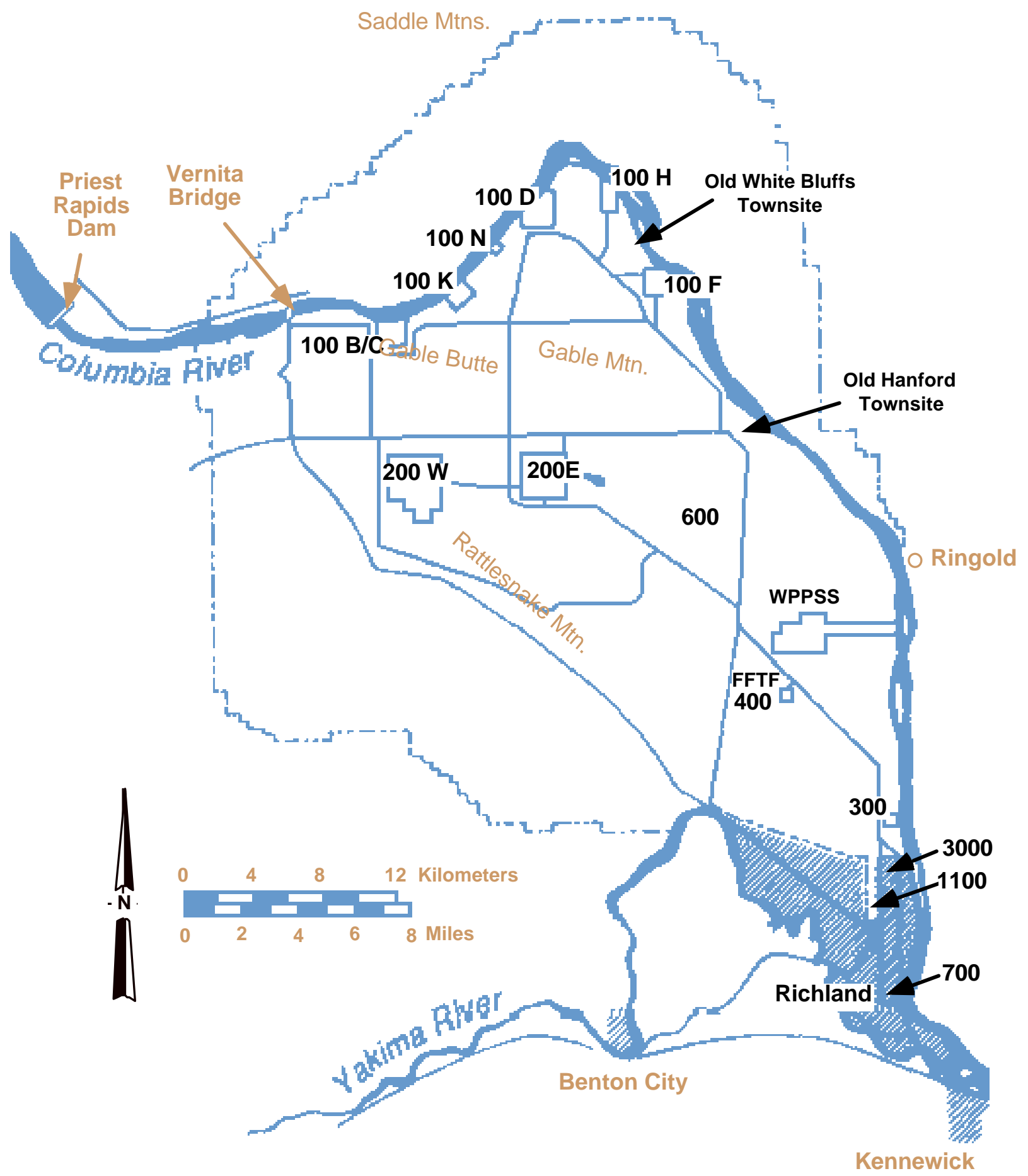

Figure 1. Map of the Hanford Site's "Areas" 
Table 1. Summary of Shallow Surface-Based Geophysics at the Hanford Site

\begin{tabular}{|c|c|c|c|c|c|}
\hline Geophysical Method & Area & Site Type & Dates & Objectives & $\begin{array}{l}\text { References } \\
\text { (Appendix B) }\end{array}$ \\
\hline \multicolumn{6}{|c|}{ Ground-Penetrating Radar } \\
\hline $\begin{array}{l}\text { Calspan Corp. with } \\
1000 \mathrm{MHz} \text { antenna }\end{array}$ & 300 & $\begin{array}{l}\text { Burial Grounds, } \\
\text { Caissons }\end{array}$ & 1975 & $\begin{array}{l}\text { Detect size, distribution, } \\
\text { and depth of buried objects }\end{array}$ & $103,104,105$ \\
\hline \multirow[t]{9}{*}{$\begin{array}{l}\text { GSSI SRI 3, 7, 8, and } \\
10 \text {, with 50-500 MHz } \\
\text { antenna }\end{array}$} & \multirow[t]{9}{*}{$\begin{array}{l}100,200, \\
300,600, \\
700, \\
1100 \\
3000\end{array}$} & \multirow{9}{*}{$\begin{array}{l}\text { Burial Grounds, Cribs, } \\
\text { Trenches, Retention } \\
\text { Basins, Disposal Pits, } \\
\text { Ditches, ISV }{ }^{(a)} \text { Test } \\
\text { Sites, Suspected } \\
\text { Waste Sites, USTs }{ }^{(b)} \text {, } \\
\text { Pipelines, Undisturbed } \\
\text { Sites }\end{array}$} & \multirow[t]{9}{*}{$\begin{array}{l}1981- \\
\text { present }\end{array}$} & $\begin{array}{l}\text { Locate and map concen- } \\
\text { trations of buried waste in } \\
\text { burial grounds, and deter- } \\
\text { mine depth of burial }\end{array}$ & $\begin{array}{l}17,19,22,23,24, \\
30,33,34,35,37 \\
39,42,45,58,60 \\
62,68,77,79,85, \\
86,90,106,110 \\
111,113,117\end{array}$ \\
\hline & & & & $\begin{array}{l}\text { Locate and map trench, } \\
\text { crib, dry well (French } \\
\text { drain), septic system } \\
\text { boundaries, and determine } \\
\text { depth of burial }\end{array}$ & $\begin{array}{l}10,12,13,15,20, \\
25,29,31,32,35, \\
36,37,38,39,40, \\
41,54,65,77,79, \\
81,83,88,93,112\end{array}$ \\
\hline & & & & $\begin{array}{l}\text { Site boreholes, soil gas } \\
\text { points, and test pits }\end{array}$ & $\begin{array}{l}2,3,4,5,6,7,9,11 \\
12,15,17,19,21 \\
22,31,38,39,59 \\
71,73,74,75,76 \\
78,80,82,84,87 \\
89,91,92,94,116 \\
117\end{array}$ \\
\hline & & & & $\begin{array}{l}\text { Locate and map pipelines } \\
\text { and utilities, and determine } \\
\text { depth of burial }\end{array}$ & $\begin{array}{l}18,28,34,36,37 \\
39,40,41,43,52 \\
110,112,118,119\end{array}$ \\
\hline & & & & $\begin{array}{l}\text { Locate and map old } \\
\text { excavations }\end{array}$ & $1,8,27,67$ \\
\hline & & & & $\begin{array}{l}\text { Locate USTs and deter- } \\
\text { mine depth }\end{array}$ & $1,8,34,43,69$ \\
\hline & & & & \begin{tabular}{|l|} 
Detect buried objects or \\
voids and determine depth
\end{tabular} & $\begin{array}{l}10,12,13,26,30 \\
31,35,39,110\end{array}$ \\
\hline & & & & $\begin{array}{l}\text { Locate, map, and charac- } \\
\text { terize geologic materials } \\
\text { and structures }\end{array}$ & $\begin{array}{l}46,47,53,55,106 \\
109\end{array}$ \\
\hline & & & & Locate possible leaks & 110 \\
\hline $\begin{array}{l}\text { Low-Frequency } \\
\text { Electromagnetic } \\
\text { Array }\end{array}$ & 300,600 & $\begin{array}{l}\text { Cold Test Site (Little } \\
\text { Egypt), Burial Ground }\end{array}$ & 1997 & Development and testing & 48 \\
\hline
\end{tabular}


Table 1. (contd)

\begin{tabular}{|c|c|c|c|c|c|}
\hline Geophysical Method & Area & Site Type & Dates & Objectives & $\begin{array}{l}\text { References } \\
\text { (Appendix B) }\end{array}$ \\
\hline \multicolumn{6}{|l|}{ Electromagnetics } \\
\hline DC Resistivity & 200 & $\begin{array}{l}\text { Cribs, Undisturbed } \\
\text { Areas }\end{array}$ & $\begin{array}{l}1982, \\
1993\end{array}$ & $\begin{array}{l}\text { Detect geologic controls } \\
\text { on groundwater movement }\end{array}$ & 96,114 \\
\hline \multirow[t]{5}{*}{$\begin{array}{l}\text { Frequency Domain } \\
\text { (e.g., Geonics EM31 } \\
\text { and EM31D) }\end{array}$} & \multirow[t]{5}{*}{$\begin{array}{l}100,200 \\
300,600 \\
1100\end{array}$} & \multirow{5}{*}{$\begin{array}{l}\text { Burial Grounds, Cribs, } \\
\text { Trenches, Retention } \\
\text { Basins, Disposal Pits, } \\
\text { Suspected Waste } \\
\text { Sites, Ditches, } \\
\text { Pipelines, Sewers, } \\
\text { ISV Test Sites, } \\
\text { Undisturbed Sites }\end{array}$} & \multirow[t]{5}{*}{$\begin{array}{l}1983- \\
\text { present }\end{array}$} & $\begin{array}{l}\text { Map concentrations of } \\
\text { buried waste }\end{array}$ & $\begin{array}{l}17,23,24,30,33, \\
34,42,86,110,113 \\
116\end{array}$ \\
\hline & & & & $\begin{array}{l}\text { Locate and map pipes, } \\
\text { utilities, tanks, and buried } \\
\text { structures/features } \\
\text { (including drums) }\end{array}$ & $\begin{array}{l}1,8,10,12,13,26, \\
30,34,35,40,42, \\
110,112,116,118\end{array}$ \\
\hline & & & & $\begin{array}{l}\text { Map location and boun- } \\
\text { daries of liquid disposal } \\
\text { trenches, cribs, pits, and } \\
\text { ditches }\end{array}$ & $\begin{array}{l}10,12,13,23,24, \\
27,30,33,34,40, \\
67,86\end{array}$ \\
\hline & & & & Identify/locate leaks & 110 \\
\hline & & & & $\begin{array}{l}\text { Detect and map clastic } \\
\text { dikes }\end{array}$ & 53 \\
\hline Time Domain & 200 & $\begin{array}{l}\text { Cribs, Undisturbed } \\
\text { Areas }\end{array}$ & 1993 & Demonstration & $46,47,55,109$ \\
\hline Induced Polarization & 200 & Crib, Undisturbed Site & 1993 & Demonstration & $46,47,55,110$ \\
\hline $\begin{array}{l}\text { Electrical Resistance } \\
\text { Tomography (ERT) }\end{array}$ & 200 & Test Sites & 1996 & $\begin{array}{l}\text { Test capabilities and } \\
\text { limitations of ERT to } \\
\text { detect and map leaks }\end{array}$ & 101,102 \\
\hline $\begin{array}{l}\text { Electromagnetic } \\
\text { Offset Logging }\end{array}$ & 300 & Burial Ground & 1999 & $\begin{array}{l}\text { Delineate depth and lateral } \\
\text { extent of conductive zones } \\
\text { (e.g., drums) }\end{array}$ & NA \\
\hline \multirow[t]{2}{*}{\begin{tabular}{|l} 
Metal Detectors (e.g., \\
Fisher TW6, \\
Metrotech 810)
\end{tabular}} & \multirow[t]{2}{*}{$\begin{array}{l}100,200, \\
300,600, \\
700, \\
1100, \\
3000\end{array}$} & Industrial Areas & $\begin{array}{l}1983- \\
\text { present }\end{array}$ & $\begin{array}{l}\text { Locate and map pipes, } \\
\text { utilities, tanks, and buried } \\
\text { structures/features } \\
\text { (including drums) }\end{array}$ & 111,118 \\
\hline & & Burial Ground & $\begin{array}{l}1975- \\
\text { present }\end{array}$ & $\begin{array}{l}\text { Determine pit boundaries } \\
\text { and distribution of buried } \\
\text { waste }\end{array}$ & $\begin{array}{l}103,104,105,106 \\
111,113\end{array}$ \\
\hline
\end{tabular}


Table 1. (contd)

\begin{tabular}{|c|c|c|c|c|c|}
\hline Geophysical Method & Area & Site Type & Dates & Objectives & $\begin{array}{c}\text { References } \\
\text { (Appendix B) }\end{array}$ \\
\hline \multicolumn{6}{|l|}{ Magnetics } \\
\hline \multirow{3}{*}{$\begin{array}{l}\text { (e.g., Geometrics } \\
\text { G816 and G856, } \\
\text { Varian VIW 2302A1, } \\
\text { Scintrex EDA Omni } \\
\text { Plus) }\end{array}$} & \multirow[t]{3}{*}{$\begin{array}{l}100,200, \\
300, \\
1100\end{array}$} & \multirow{3}{*}{$\begin{array}{l}\text { Burial Grounds, } \\
\text { Disposal Pits, Cribs/ } \\
\text { Undisturbed areas, } \\
\text { ISV Test Site }\end{array}$} & \multirow[t]{3}{*}{$\begin{array}{l}1975- \\
\text { present }\end{array}$} & $\begin{array}{l}\text { Determine pit boundaries } \\
\text { and distribution of buried } \\
\text { waste }\end{array}$ & $\begin{array}{l}17,45,54,105,111 \\
117\end{array}$ \\
\hline & & & & $\begin{array}{l}\text { Locate and map pipes, } \\
\text { utilities, and tanks }\end{array}$ & NA \\
\hline & & & & Geologic Controls & 96,114 \\
\hline \multicolumn{6}{|l|}{ Seismic } \\
\hline $\begin{array}{l}\text { Vertical Seismic } \\
\text { Profiling }\end{array}$ & 200 & $\begin{array}{l}\text { Cribs, Undisturbed } \\
\text { Areas }\end{array}$ & 1994 & $\begin{array}{l}\text { Test techniques for } \\
\text { obtaining velocity control }\end{array}$ & 101 \\
\hline Refraction & 200,300 & $\begin{array}{l}\text { Cribs, Undisturbed } \\
\text { Areas, Burial Grounds }\end{array}$ & $\begin{array}{l}1975 \\
1993\end{array}$ & Hydrogeology & $46,47,55,109$ \\
\hline Reflection & $\begin{array}{l}200,300 \\
600\end{array}$ & $\begin{array}{l}\text { Cribs, Undisturbed } \\
\text { Areas, Burial Grounds }\end{array}$ & $\begin{array}{l}1975 \\
1991 \\
1993\end{array}$ & Hydrogeology & $\begin{array}{l}46,47,55,63,64 \\
97,99,100,109\end{array}$ \\
\hline $\begin{array}{l}\text { 2-D High Resolution } \\
\text { Reflection with } \\
\text { amplitude offset } \\
\text { technology }\end{array}$ & 200 & Cribs & 1999 & $\begin{array}{l}\text { Determine location and } \\
\text { distribution of DNAPL }^{(c)}\end{array}$ & NA \\
\hline $\begin{array}{l}\text { 3-D Holographic } \\
\text { Imaging }\end{array}$ & 300 & Burial Grounds & 1980 & $\begin{array}{l}\text { Locate map and define } \\
\text { buried waste }\end{array}$ & 105 \\
\hline \multicolumn{6}{|l|}{ Gravity } \\
\hline $\begin{array}{l}\text { Worden Gravity } \\
\text { Meter }\end{array}$ & 200 & Undisturbed & 1982 & Hydrogeology & 96,114 \\
\hline \multicolumn{6}{|c|}{$\begin{array}{ll}\text { (a) In situ vitrification. } \\
\text { (b) Underground storage tanks. } \\
\text { (c) Dense nonaqueous-phase liquid. }\end{array}$} \\
\hline
\end{tabular}

\section{Ground-Penetrating Radar (GPR)}

GPR is by far the most widely used geophysical method at the Hanford Site. This technique is used primarily for the detection and mapping of underground pipelines, utilities, buried debris, and other structures that might interfere with or impact drilling or other intrusive characterization or remediation methods. A 300-MHz antenna has generally been preferred with a maximum depth of investigation of less than $\sim 16 \mathrm{ft}$.

Collins (1995) (B-48) recently demonstrated holographic imaging technology at a test site near the 618-10 burial ground (a.k.a., Little Egypt) and at the 618-4 burial ground. 


\section{Electromagnetics}

The most widely used electromagnetic method has been Frequency-Domain electromagnetics (FDEM) - primarily using the Geonics Ground Conductivity Meter (EM-31 or -31D). Metal detectors (and Time-Domain Electromagnetics [TDEM]) are also frequently used. Again the primary use of these techniques has been for detection and mapping of underground pipelines, utilities, buried debris, and other structures - primarily metallic or conductive materials. The depth of investigation with these techniques is believed to be relatively shallow $(<30 \mathrm{ft})$.

Lewis and Teel (1994) (B-66), suggested that electrical resistance tomography (ERT) might be the most promising technology for leak detection. Narbutovskih and others (1996) (B-101 and -102) demonstrated ERT's ability to provide early leak detection and the ability to install the needed electrode arrays using cone-penetrometer technology.

\section{Magnetics}

Total field magnetic surveys have been used extensively for the detection and mapping of buried waste and other ferrous objects. Recently, interest has been sparked regarding the potential for adaptation of this technology to estimate the number of buried metallic drums at a site.

\section{Seismic Reflection}

Narbutovskih (1993) (B-97) found that new seismic reflection technology has the potential for providing the greatest resolution of the subsurface hydrogeology than any other geophysical technique. However, only very limited testing of these technologies have been performed.

Earth Sciences and Resources Institute (University of South Carolina) staff recently tested the ability of seismic reflection and amplitude verses offset (AVO) analysis techniques to determine the location and distribution of dense nonaqueous-phase liquids (DNAPLs) near the 216-Z-9 Trench.

\section{Borehole Geophysical Logging Methods}

Borehole geophysical logging methods have been used at the Hanford Site since the mid- to late1940s. The Hanford formation and most of the Ringold Formation that make up the vadose zone at the Hanford Site are unconsolidated, non-lithified, and non-cemented. Thus, steel cased boreholes have been the norm at the site since the 1940s. The casing, and in later years a grout annular seal, have been the biggest factors in determining the types of logging methods used. Traditionally, gross gamma ray and neutron moisture have been the two most commonly used downhole tools. Recently, several other techniques have been tested and/or used for contaminant and lithologic characterization in the subsurface. 
Table 2 is a list of logging types used at the Hanford Site, the approximate dates they were used, and references that either present data and data interpretation or discuss instrument design, calibration, or some other aspect of the pertinent logging type. Also attached (Appendix C) is a bibliography of borehole geophysics used at the Hanford Site. The reference numbers on Table 2 correspond to those in the bibliography. Unfortunately, most older logging data have not been reported, but there is a lot of information on the more recent logging efforts. References C-10, -26 through $-32,-83,-84,-126,-155$, and -182 through -184 are compilations of logging data and are not included in Table 2.

\section{Gross Gamma-Ray Logging}

The earliest logging at Hanford was gross gamma-ray logging at liquid waste disposal sites to monitor the subsurface distribution of gamma-emitting contaminants. Later, gross gamma-ray logging at tank farms was initiated as a secondary method for leak detection. Gross gamma-ray logging at tank farms stopped in 1994. The earliest logs were obtained with Geiger-Mueller detectors (later logging in highcount rate zones also used GM detectors). Later logs were obtained with scintillation detectors.

A second major use of gross gamma-ray logging has been lithologic characterization. For the past several years, new boreholes are routinely logged for this purpose. More frequently, the old gross-gamma logs are being incorporated into geologic interpretations of the subsurface resulting in improved conceptual models.

\section{Spectral Gamma-Ray Logging}

Spectral gamma-ray logging has replaced gross gamma-ray logging at the Hanford Site for most applications. Spectral gamma-ray logging was developed in the 1970s, used sporadically in the 1980s, and used much more intensely in the 1990s. The dominant use has been contaminant monitoring and characterization for waste management and environmental restoration purposes. Spectral gamma-ray logging has also been used for lithologic characterization in new and uncontaminated boreholes (by summing the potassium, uranium, and thorium channels to obtain a natural gamma log). A third major use of spectral gamma-ray logging has been facility support, such as determining subsurface conditions before constructing new transfer pipes.

Spectral gamma-ray logging was initiated at tank farms in 1995 and used until 2000 to log essentially every borehole within the farms for baseline characterization.

\section{Neutron Moisture Logging}

The second most used logging tools at the Hanford Site are neutron moisture tools. Neutron moisture logging was used as early as the 1960s for contaminant monitoring at liquid waste disposal facilities and lithologic characterization. Another early use of the logging data was measurement of moisture to aid determination of soil thermal conductivity to evaluate heat dissipation (heat from radioactive decay) (C-141). The early neutron probe used a Pu-Be source for neutrons. However, its use was discontinued 
Table 2. Summary of Borehole Geophysical Logging Methods Used at Hanford

\begin{tabular}{|c|c|c|c|}
\hline Log Type & Use & Dates & References (Appendix C) \\
\hline Geiger-Mueller tube & $\begin{array}{l}100 \text { Areas } \\
200 \text { Areas }\end{array}$ & $1947-1953$ & $\begin{array}{l}\text { Data not reported or reported only in } \\
\text { monthly status reports }\end{array}$ \\
\hline $\begin{array}{l}\text { Prototype gamma } \\
\text { scintillation probe }\end{array}$ & $\begin{array}{l}200 \text { Areas liquid waste } \\
\text { disposal sites }\end{array}$ & 1954 & \\
\hline $\begin{array}{l}2^{\text {nd }} \text { generation gamma } \\
\text { scintillation logging } \\
\text { system }\end{array}$ & $\begin{array}{l}200 \text { Areas liquid waste } \\
\text { disposal sites }\end{array}$ & $1955-1961$ & $1,2,44,126,145$ \\
\hline $\begin{array}{l}3^{\text {rd }} \text { generation gamma } \\
\text { scintillation logging } \\
\text { system }\end{array}$ & $\begin{array}{l}\text { Contaminant monitoring } \\
\text { at liquid waste disposal } \\
\text { sites }\end{array}$ & $1961-1973$ & $1,2,44,126,127,156$ \\
\hline \multirow{2}{*}{$\begin{array}{l}4^{\text {th }} \text { generation gamma } \\
\text { scintillation logging } \\
\text { system (Gearhart-Owen } \\
\text { Industries Model } 3200)\end{array}$} & $\begin{array}{l}\text { Contaminant monitoring } \\
\text { at liquid waste disposal } \\
\text { sites }\end{array}$ & $\begin{array}{l}1974 \text { - early } \\
1990 \text { s }\end{array}$ & $33,69,83,158$ \\
\hline & $\begin{array}{l}\text { Lithologic } \\
\text { characterization }\end{array}$ & $\begin{array}{l}1974 \text { - early } \\
1990 \text { s }\end{array}$ & $\begin{array}{l}3,4,5,6,7,8,9,12,24,25,34,56,57 \\
60,61,73,98,99,143,144,150,151, \\
163,164\end{array}$ \\
\hline $\begin{array}{l}\text { Gross gamma-ray logging } \\
\text { (Geiger-Mueller tubes) }\end{array}$ & Tank farm monitoring & $1961-1994$ & \\
\hline $\begin{array}{l}\text { Gross gamma-ray logging } \\
\text { (Scintillation probe) }\end{array}$ & Tank farm monitoring & $1975-1994$ & $13,87,88,114,116,118,123,183$ \\
\hline $\begin{array}{l}\text { Development Vans } \\
\text { (spectral gamma logging } \\
\text { system) }\end{array}$ & Contaminant monitoring & Early 1970s & 23 \\
\hline $\begin{array}{l}\text { Mobile Radionuclide } \\
\text { Analysis Laboratory } \\
\text { (spectral gamma) }\end{array}$ & Contaminant monitoring & $\sim 1982-1988$ & $17,19,20,146,173$ \\
\hline \multirow[t]{6}{*}{$\begin{array}{l}\text { Radionuclide Logging } \\
\text { System (HPGe and NaI } \\
\text { spectral gamma) }\end{array}$} & $\begin{array}{l}\text { Contaminant monitoring } \\
\text { and characterization }\end{array}$ & 1993 - present & $\begin{array}{l}36,62,64,65,66,67,68,70,76,78 \\
79,86,117,121,122,142,147,148, \\
149,153,174,177,178\end{array}$ \\
\hline & $\begin{array}{l}\text { Interim Response Action } \\
\left(\mathrm{CCl}_{4}\right)\end{array}$ & 1991 & 77 \\
\hline & $\begin{array}{l}\text { Lithologic } \\
\text { characterization }\end{array}$ & 1990 - present & $\begin{array}{l}70,75,86,100,106,107,108,109 \\
131,152,179\end{array}$ \\
\hline & CERCLA characterization & 1992 - present & $\begin{array}{l}15,16,43,45,46,49,51,52,53,63, \\
81,82,112,113,115,132,154,158, \\
159,160\end{array}$ \\
\hline & Facility support & $1992-1998$ & $\begin{array}{l}35,42,47,48,50,54,58,101,111 \\
119,176\end{array}$ \\
\hline & $\begin{array}{l}\text { Tank farm } \\
\text { characterization }\end{array}$ & 1995 to 2000 & $\begin{array}{l}14,80,89,90,91,92,93,124,134, \\
135,136,137,138,158,161,162,163, \\
164,165,166,167,168,169,170,171, \\
172,184\end{array}$ \\
\hline
\end{tabular}


Table 2. (contd)

\begin{tabular}{|c|c|c|c|}
\hline Log Type & Use & Dates & References (Appendix C) \\
\hline \multirow[t]{3}{*}{$\begin{array}{l}\text { Neutron (moisture) } \\
\text { logging }\end{array}$} & Contaminant monitoring & $1963-1985$ & $\begin{array}{l}\text { Data generally not reported. Original } \\
\text { logs available. } \\
141\end{array}$ \\
\hline & $\begin{array}{l}\text { Lithologic } \\
\text { characterization }\end{array}$ & 1980s - present & $\begin{array}{l}8,9,40,41,56,57,70,97,108,109 \\
131,182\end{array}$ \\
\hline & Facility support & 1998 & 101 \\
\hline Neutron flux logging & $\begin{array}{l}\text { Contaminant } \\
\text { characterization }\end{array}$ & 1981,1982 & 71,72 \\
\hline $\begin{array}{l}\text { Prompt Fission Neutron } \\
\text { Logging }\end{array}$ & $\begin{array}{l}\text { Contamination } \\
\text { characterization }\end{array}$ & $\begin{array}{l}1978,1984, \\
1993\end{array}$ & 59,74 \\
\hline $\begin{array}{l}\text { Neutron - gamma logging } \\
\text { (Cf source) }\end{array}$ & $\begin{array}{l}\text { Contamination } \\
\text { characterization }\end{array}$ & 1999 & Data not yet available. \\
\hline Downhole Seismic & Testing and development & $1994-1995$ & $37,38,39,103$ \\
\hline $\begin{array}{l}\text { Electrical Resistivity } \\
\text { Tomography }\end{array}$ & Testing and development & 1996 & $104,105,120$ \\
\hline Temperature logging & $\begin{array}{l}\text { Numerous Hanford Site } \\
\text { wells for contaminant } \\
\text { monitoring }\end{array}$ & $\begin{array}{l}1961-1974 \\
1974-\sim 1982 \\
1999\end{array}$ & $\begin{array}{l}\text { Data not reported or reported in } \\
\text { monthly status reports. Original logs } \\
\text { available. } \\
125\end{array}$ \\
\hline $\begin{array}{l}\text { Spontaneous } \\
\text { Potential/Resistivity }\end{array}$ & $\begin{array}{l}\text { Very few selected wells } \\
\text { and boreholes }\end{array}$ & $1979-\sim 1987$ & $\begin{array}{l}\text { Data not reported. Original logs } \\
\text { available. }\end{array}$ \\
\hline Caliper & $\begin{array}{l}\text { Few selected wells and } \\
\text { boreholes }\end{array}$ & $1978-\sim 1990$ & $\begin{array}{l}\text { Data not reported. Original logs } \\
\text { available. }\end{array}$ \\
\hline Magnetic logging & $\begin{array}{l}\text { Very few selected, deep } \\
\text { wells and boreholes }\end{array}$ & $1981-1984$ & $\begin{array}{l}\text { Data not reported. Original logs } \\
\text { available }\end{array}$ \\
\hline Density & $\begin{array}{l}\text { Numerous wells and } \\
\text { boreholes for lithologic } \\
\text { characterization }\end{array}$ & $1976-1989$ & $\begin{array}{l}\text { Data generally not reported. Original } \\
\text { logs available. } \\
8,9,56,57\end{array}$ \\
\hline Sonic logging & $\begin{array}{l}\text { Very few selected wells } \\
\text { and boreholes }\end{array}$ & $1979-1985$ & $\begin{array}{l}\text { Data not reported. Original logs } \\
\text { available. }\end{array}$ \\
\hline Miscellaneous Logs & Very few selected wells & $1967-1995$ & $\begin{array}{l}\text { Data not reported. Original } \\
\text { Schlumberger, Hallibuton, and } \\
\text { Birdwell logs available. }\end{array}$ \\
\hline Indium and copper foils & $\begin{array}{l}216-Z-1 A \text { crib } \\
216-Z-12 \text { crib }\end{array}$ & $\begin{array}{l}1983 \\
1981,1989\end{array}$ & $19,71,72$ \\
\hline $\begin{array}{l}\text { Other nuclear logging } \\
\text { techniques }\end{array}$ & $\begin{array}{l}\text { Experimental and } \\
\text { development }\end{array}$ & $1980 \mathrm{~s}$ & $20,21,22,94,95,129,130,139$ \\
\hline
\end{tabular}


when more stringent radiation protection measures were implemented that made it difficult to shield operating personnel (C-128). The probe in use today uses an Am-Be source and logging focuses on lithologic characterization, although some logging for facility support has been done.

\section{Temperature Logging}

Temperature logging was done extensively in the 1960s and 1970s at the Hanford Site. Unfortunately, no documentation of data or data interpretation was found during this compilation of geophysical logging. However, many, if not most, of the original logs exist in the geophysics library at PNNL. The logging system was used to log essentially every well on the Hanford Site before the early 1970s (C-128). The primary use of the data was to utilize the heat of disposed liquid wastes as a tracer to determine subsurface movement of the waste.

\section{Other Geophysical Borehole Logging}

Several other types of geophysical borehole logging were done in the past at the Hanford Site. Monitoring of neutron flux down boreholes and prompt fission neutron logging were done to characterize radionuclide contaminants. Spontaneous potential/resistivity logging, caliper logging, magnetic logging, and sonic logging have all been done for lithologic characterization. These latter methods have been used primarily in deep boreholes into basalt where no casing is required. All of these methods have been used in only a few boreholes and, for the most part, the data and their interpretation are not reported.

Most recently, three new or revived logging methods have been tested at the Hanford Site. These include neutron-gamma logging for specific elemental analysis, temperature logging, and spectral gamma-ray logging with new detectors designed to function in zones of high count rates in which previous detectors were saturated. The results of these logging efforts have not yet been published but should be available in fiscal year 2000 . 


\section{Appendix A}

Catalog of Shallow Surface Geophysical Surveys Arranged by Site 


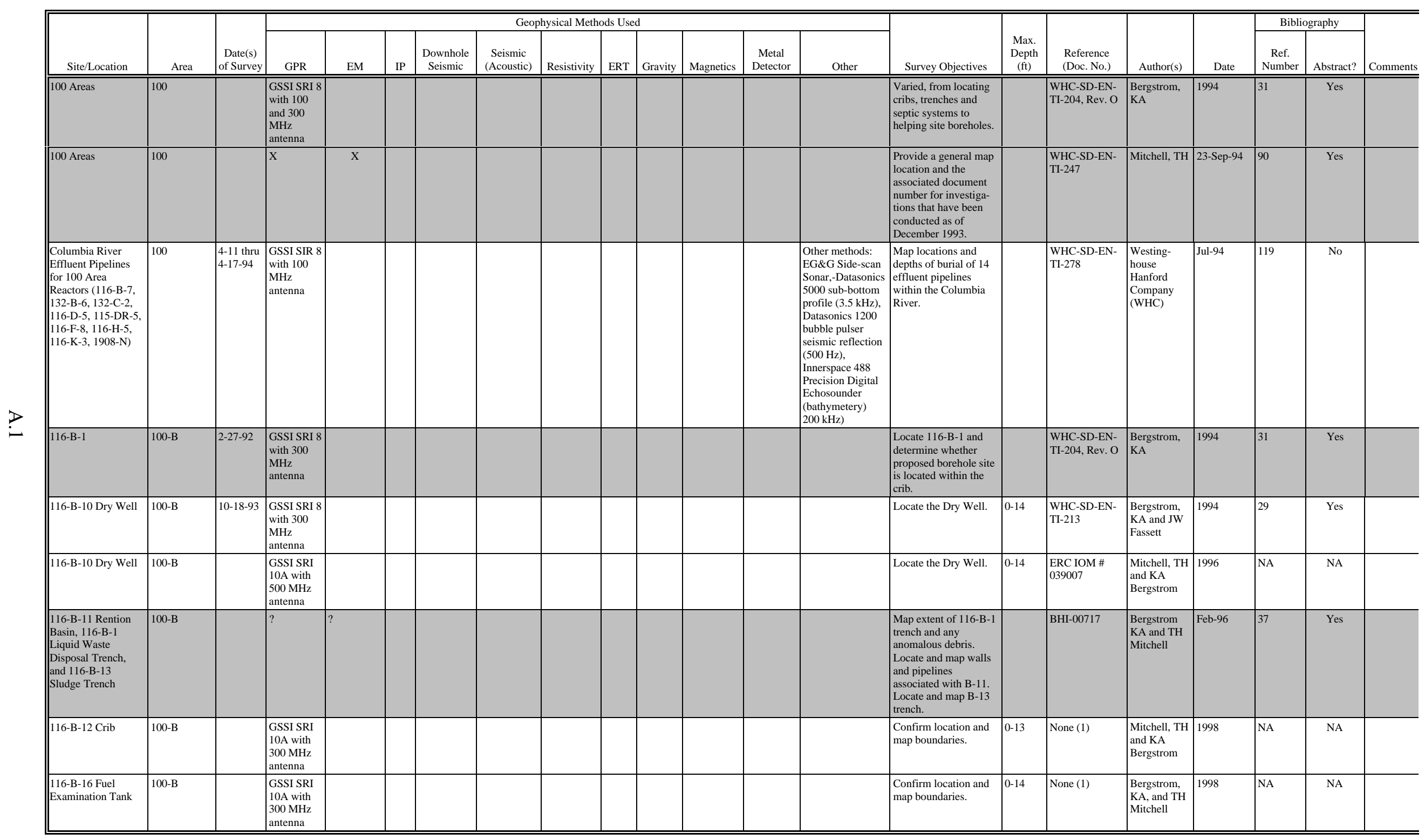




\begin{tabular}{|c|c|c|c|c|c|c|c|c|c|c|c|c|c|c|c|c|c|c|c|c|c|}
\hline \multirow[b]{2}{*}{ Site/Location } & \multirow[b]{2}{*}{ Area } & \multirow[b]{2}{*}{$\begin{array}{c}\text { Date(s) } \\
\text { of Survey }\end{array}$} & \multicolumn{11}{|c|}{ Geophysical Methods Used } & \multirow[b]{2}{*}{ Survey Objectives } & \multirow[b]{2}{*}{\begin{tabular}{|l} 
Max. \\
Depth \\
(ft) \\
\end{tabular}} & \multirow[b]{2}{*}{$\begin{array}{l}\text { Reference } \\
\text { (Doc. No.) }\end{array}$} & \multirow[b]{2}{*}{ Author(s) } & \multirow[b]{2}{*}{ Date } & \multicolumn{2}{|c|}{ Bibliography } & \multirow[b]{2}{*}{ Comments } \\
\hline & & & GPR & EM & IP & $\begin{array}{c}\text { Downhole } \\
\text { Seismic }\end{array}$ & $\begin{array}{c}\text { Seismic } \\
\text { (Acoustic) }\end{array}$ & Resistivity & ERT & Gravity & Magnetics & $\begin{array}{c}\text { Metal } \\
\text { Detector }\end{array}$ & Other & & & & & & \begin{tabular}{|c|} 
Ref. \\
Number
\end{tabular} & Abstract? & \\
\hline $\begin{array}{l}\text { 116-B-2 Fuel } \\
\text { Storage Basin } \\
\text { Trench }\end{array}$ & $100-\mathrm{B}$ & & \begin{tabular}{|l|} 
GSSI SRI 8 \\
with 300 \\
MHz \\
antenna \\
\end{tabular} & & & & & & & & & & & Locate and map trench. & $0-12$ & \begin{tabular}{|l|} 
ERC IOM \# \\
039006
\end{tabular} & \begin{tabular}{|l|} 
Bergstrom, \\
KA, and TH \\
Mitchell
\end{tabular} & 1992 & $\mathrm{NA}$ & $\mathrm{NA}$ & \\
\hline $\begin{array}{l}\text { 116-B-2 Fuel } \\
\text { Storage Basin } \\
\text { Trench }\end{array}$ & $100-\mathrm{B}$ & & \begin{tabular}{|l|} 
GSSI SRI \\
$10 \mathrm{~A}$ with \\
$300 \mathrm{MHz}$ \\
antenna \\
\end{tabular} & & & & & & & & & & & Locate and map trench. & $0-12$ & $\begin{array}{l}\text { ERC IOM \# } \\
039007\end{array}$ & \begin{tabular}{|l|} 
Bergstrom, \\
KA, and TH \\
Mitchell
\end{tabular} & 1996 & $\mathrm{NA}$ & $\mathrm{NA}$ & \\
\hline 116-B-3 Pluto Crib & $100-\mathrm{B}$ & & \begin{tabular}{|l|} 
GSSI SRI 8 \\
with 300 \& \\
$500 \mathrm{MHz}$ \\
antenna
\end{tabular} & & & & & & & & & & & $\begin{array}{l}\text { Confirm location and } \\
\text { map boundaries. }\end{array}$ & $14-16$ & BHI-00344 & \begin{tabular}{|l|} 
Mitchell, TH \\
and KA \\
Bergstrom
\end{tabular} & \begin{tabular}{|l|l|} 
Jul-95 & \\
\end{tabular} & 93 & No & \\
\hline $\begin{array}{l}\text { 116-B-4 French } \\
\text { Drain }\end{array}$ & $100-\mathrm{B}$ & & \begin{tabular}{|l|} 
GSSI SRI 8 \\
with 300 \& \\
$500 \mathrm{MHz}$ \\
antenna
\end{tabular} & & & & & & & & & & & $\begin{array}{l}\text { Confirm location and } \\
\text { map boundaries. }\end{array}$ & $14-16$ & BHI-00344 & \begin{tabular}{|l|} 
Mitchell, TH \\
and KA \\
Bergstrom
\end{tabular} & \begin{tabular}{|l|l|} 
Jul-95 \\
\end{tabular} & 93 & No & \\
\hline $116-\mathrm{B}-5$ & $100-\mathrm{B}$ & $2-21-92$ & \begin{tabular}{|l|} 
GSSI SRI 8 \\
with 300 \\
MHz \\
antenna
\end{tabular} & & & & & & & & & & & \begin{tabular}{|l|} 
Delineate crib \\
boundaries. If possible \\
determine if any areas \\
had caved in.
\end{tabular} & & $\begin{array}{l}\text { WHC-SD-EN- } \\
\text { TI-204, Rev. O }\end{array}$ & $\begin{array}{l}\text { Bergstrom, } \\
\text { KA }\end{array}$ & 1994 & 31 & Yes. & \\
\hline 116-B-6-1 Crib & $100-\mathrm{B}$ & & $\mathrm{x}$ & & & & & & & & & & & $\begin{array}{l}\text { ISV site. Locate crib, } \\
\text { determine size and } \\
\text { configuration. Detect } \\
\text { nearby objects or } \\
\text { voids. }\end{array}$ & $20 \mathrm{ft}$ & $\begin{array}{l}\text { PNL Memo to } \\
\text { S.J. Mitchell }\end{array}$ & \begin{tabular}{|l|} 
G.A. \\
Sandness
\end{tabular} & 02-Aug-88 & $\mathrm{NA}$ & $\mathrm{NA}$ & \\
\hline 116-B-6A Crib & $100-\mathrm{B}$ & & $\begin{array}{l}\text { GSSI SRI } \\
10 \mathrm{~A} \text { with } \\
300 \mathrm{MHz} \\
\text { antenna }\end{array}$ & & & & & & & & & & & $\begin{array}{l}\text { Confirm location and } \\
\text { map boundaries. }\end{array}$ & & $\begin{array}{l}\text { ERC IOM } \\
\# 039006\end{array}$ & \begin{tabular}{|l|} 
Mitchell, TH \\
and KA \\
Bergstrom
\end{tabular} & 1998 & $\mathrm{NA}$ & $\mathrm{NA}$ & \\
\hline 116-B-6B Crib & $100-\mathrm{B}$ & & \begin{tabular}{|l} 
GSSI SRI \\
$10 \mathrm{~A}$ with \\
$500 \mathrm{MHz}$ \\
antenna \\
\end{tabular} & & & & & & & & & & & $\begin{array}{l}\text { Confirm location and } \\
\text { map boundaries. }\end{array}$ & & $\begin{array}{l}\text { ERC IOM } \\
\# 039006\end{array}$ & \begin{tabular}{|l|} 
Mitchell, TH \\
and KA \\
Bergstrom
\end{tabular} & 1996 & NA & $\mathrm{NA}$ & \\
\hline 116-B-6B Crib & $100-\mathrm{B}$ & & \begin{tabular}{|l|} 
GSSI SRI \\
$10 \mathrm{~A}$ with \\
$300 \mathrm{MHz}$ \\
antenna \\
\end{tabular} & & & & & & & & & & & $\begin{array}{l}\text { Confirm location and } \\
\text { map boundaries. }\end{array}$ & & $\begin{array}{l}\text { ERC IOM } \\
\# 039007\end{array}$ & \begin{tabular}{|l|} 
Mitchell, TH \\
and KA \\
Bergstrom
\end{tabular} & 1998 & $\mathrm{NA}$ & NA & \\
\hline $\begin{array}{l}\text { 116-B-9 French } \\
\text { Drain }\end{array}$ & $100-\mathrm{B}$ & $10-18-93$ & \begin{tabular}{|l|}
$\begin{array}{l}\text { GSSI SRI } 8 \\
\text { with } 300 \\
\text { antenna }\end{array}$ \\
\end{tabular} & & & & & & & & & & & $\begin{array}{l}\text { Locate the French } \\
\text { Drain. }\end{array}$ & & $\begin{array}{l}\text { WHC-SD-EN- } \\
\text { TI-213 }\end{array}$ & \begin{tabular}{|l|} 
Bergstrom, \\
KA and JW \\
Fassett \\
\end{tabular} & 1994 & 29 & Yes. & \\
\hline $\begin{array}{l}\text { 116-B-9 French } \\
\text { Drain }\end{array}$ & $100-\mathrm{B}$ & & \begin{tabular}{|l|} 
GSSI SRI \\
$10 \mathrm{~A}$ with \\
$500 \mathrm{MHz}$ \\
antenna
\end{tabular} & & & & & & & & & & & $\begin{array}{l}\text { Locate the French } \\
\text { Drain. }\end{array}$ & & $\begin{array}{l}\text { ERC IOM \# } \\
039006\end{array}$ & \begin{tabular}{|l|} 
Mitchell, TH \\
and KA \\
Bergstrom
\end{tabular} & 1996 & $\mathrm{NA}$ & $\mathrm{NA}$ & \\
\hline 118-B-1 & $100-\mathrm{B}$ & $4-6-93$ & \begin{tabular}{|l|} 
GSSI SIR 8 \\
with 300 \\
MHz \\
antenna
\end{tabular} & $\begin{array}{l}\text { Geonics } \\
\text { EM-31D }\end{array}$ & & & & & & & $\mathrm{x}$ & & & $\begin{array}{l}\text { Map the concentrations } \\
\text { of waste and aid in the } \\
\text { selection of test pit } \\
\text { excavation locations. }\end{array}$ & $10-12 \mathrm{ft}$ & $\begin{array}{l}\text { WHC-SD-EN- } \\
\text { TI-137 (1). } \\
\text { DOE/RL-95-34 } \\
\text { (2). WHC-SD- } \\
\text { EN-DP-066 (3.) } \\
\text { WHC-SD-EN- } \\
\text { TI-138 (4) }\end{array}$ & \begin{tabular}{|l|} 
1) \\
Bergstrom, \\
KA; TH \\
Mitchell and \\
AL \\
Langstaff. \\
2) DOE. 3) \\
Berstrom and \\
Mitchell. \\
4) Bergstrom \\
and Mitchell \\
\end{tabular} & \begin{tabular}{|l|} 
1) Oct-93 \\
2)Aug-95. \\
3) Feb- \\
1994. \\
4) Nov-93
\end{tabular} & $\begin{array}{l}\text { 1) } 17 . \\
\text { 2) } 117 . \\
\text { 3) } 22 . \\
\text { 4) } 19\end{array}$ & $\begin{array}{l}\text { 1) No. } \\
\text { 2) Yes. } \\
\text { 3) no. } \\
\text { 4) no }\end{array}$ & \\
\hline
\end{tabular}




\begin{tabular}{|c|c|c|c|c|c|c|c|c|c|c|c|c|c|c|c|c|c|c|c|c|c|}
\hline \multirow[b]{2}{*}{ Site/Location } & \multirow[b]{2}{*}{ Area } & \multirow[b]{2}{*}{$\begin{array}{c}\text { Date(s) } \\
\text { of Survey }\end{array}$} & \multicolumn{11}{|c|}{$\begin{array}{l}\text { Geophysical Methods Used } \\
\end{array}$} & \multirow[b]{2}{*}{ Survey Objectives } & \multirow[b]{2}{*}{\begin{tabular}{|l} 
Max. \\
Depth \\
(ft)
\end{tabular}} & \multirow[b]{2}{*}{$\begin{array}{l}\text { Reference } \\
\text { (Doc. No.) }\end{array}$} & \multirow[b]{2}{*}{ Author(s) } & \multirow[b]{2}{*}{ Date } & \multicolumn{2}{|c|}{ Bibliography } & \multirow[b]{2}{*}{ Comments } \\
\hline & & & GPR & EM & IP & \begin{tabular}{|c|} 
Downhole \\
Seismic
\end{tabular} & $\begin{array}{c}\text { Seismic } \\
\text { (Acoustic) }\end{array}$ & Resistivity & ERT & Gravity & Magnetics & $\begin{array}{l}\text { Metal } \\
\text { Detector }\end{array}$ & Other & & & & & & $\begin{array}{c}\text { Ref. } \\
\text { Number }\end{array}$ & Abstract? & \\
\hline 130-B-1 & $100-\mathrm{B}$ & & $\bar{x}$ & & & & & & & & & $\bar{x}$ & & $\begin{array}{l}\text { Locate and map } \\
\text { underground fuel } \\
\text { storage tanks, pipes, } \\
\text { and cables. }\end{array}$ & $15 \mathrm{ft}$ & $\begin{array}{l}\text { PNL letter } \\
\text { report to Ron } \\
\text { Shuck, WHO }\end{array}$ & $\begin{array}{l}\text { G.A. } \\
\text { Sandness }\end{array}$ & 27 -Sep-90 & $\mathrm{NA}$ & $\overline{\mathrm{NA}}$ & \\
\hline 199-B2-12 & 100-B & $2-6-92$ & \begin{tabular}{|l|} 
GSSI SRI 8 \\
with 100 \\
MHz \\
antenna
\end{tabular} & & & & & & & & & & & \begin{tabular}{|l|} 
Locate utilities and \\
buried debris that may \\
affect drilling of \\
borehole.
\end{tabular} & & $\begin{array}{l}\text { WHC-SD-EN- } \\
\text { TI-204, Rev. O }\end{array}$ & \begin{tabular}{|l} 
Bergstrom, \\
KA
\end{tabular} & 1994 & 31 & Yes. & \\
\hline $\begin{array}{l}\text { 199-B4-9 and 116- } \\
\text { B-2 }\end{array}$ & $100-\mathrm{B}$ & $3-5-92$ & \begin{tabular}{|l|} 
GSSI SRI 8 \\
with 300 \\
MHz \\
antenna
\end{tabular} & & & & & & & & & & & $\begin{array}{l}\text { Locate utilities and } \\
\text { buried debris that may } \\
\text { affect drilling of } \\
\text { borehole B4-9 and } \\
\text { locate 116-B-2. }\end{array}$ & & $\begin{array}{l}\text { WHC-SD-EN- } \\
\text { TI-204, Rev. O }\end{array}$ & \begin{tabular}{|l} 
Bergstrom, \\
KA
\end{tabular} & 1994 & 31 & Yes. & \\
\hline 199-B5-2 & $100-\mathrm{B}$ & $\begin{array}{l}3-11 / 12- \\
92\end{array}$ & \begin{tabular}{|l|} 
GSSI SRI 8 \\
with 300 \\
MHz \\
antenna
\end{tabular} & & & & & & & & & & & $\begin{array}{l}\text { Locate utilities and } \\
\text { buried debris that may } \\
\text { affect drilling of } \\
\text { borehole. }\end{array}$ & & $\begin{array}{l}\text { WHC-SD-EN- } \\
\text { TI-204, Rev. O }\end{array}$ & $\begin{array}{l}\text { Bergstrom, } \\
\text { KA }\end{array}$ & 1994 & 31 & Yes. & \\
\hline 199-B9-2 & 100-B & $2-28-92$ & \begin{tabular}{|l|} 
GSSI SRI I \\
with 300 \\
MHz \\
antenna
\end{tabular} & & & & & & & & & & & $\begin{array}{l}\text { Locate utilities and } \\
\text { buried debris that may } \\
\text { affect drilling of } \\
\text { borehole. }\end{array}$ & & $\begin{array}{l}\text { WHC-SD-EN- } \\
\text { TI-204, Rev. O }\end{array}$ & $\begin{array}{l}\text { Bergstrom, } \\
\text { KA }\end{array}$ & 1994 & 31 & Yes. & \\
\hline 116-C-2A Pluto Crib & $100-\mathrm{C}$ & $5-14-93$ & \begin{tabular}{|l|} 
GSSI SRI 8 \\
with 300 \\
antenna
\end{tabular} & & & & & & & & & & & $\begin{array}{l}\text { Map crib boundaries } \\
\text { and locate sampling } \\
\text { borehole 199-B9-4. }\end{array}$ & & $\begin{array}{l}\text { WHC-SD-EN- } \\
\text { TI-154 }\end{array}$ & \begin{tabular}{|l|} 
Bergstrom, \\
Mitchell, and \\
Kiesler
\end{tabular} & Jul-93 & 15 & No. & \\
\hline $\begin{array}{l}\text { 116-C-2B Pluto Crib } \\
\text { Pump Station }\end{array}$ & $100-\mathrm{C}$ & & \begin{tabular}{|l|} 
GSSI SRI \\
$10 \mathrm{~A}$ with \\
$300 \mathrm{MHz}$ \\
antenna \\
\end{tabular} & \begin{tabular}{|l|} 
Geonics \\
EM-31
\end{tabular} & & & & & & & & & & $\begin{array}{l}\text { Map associated pipes } \\
\text { and utilities and } \\
\text { unknown buried } \\
\text { structures. }\end{array}$ & & None (1) & \begin{tabular}{|l|} 
Mitchell, TH \\
and KA \\
Bergstrom
\end{tabular} & 1998 & $\mathrm{NA}$ & $\mathrm{NA}$ & \\
\hline $\begin{array}{l}\text { 116-C-2C Pluto Crib } \\
\text { Sand Filter }\end{array}$ & $100-\mathrm{C}$ & & \begin{tabular}{|l|} 
GSSI SRI \\
$10 \mathrm{~A}$ with \\
$300 \mathrm{MHz}$ \\
antenna
\end{tabular} & \begin{tabular}{|l|} 
Geonics \\
EM-32
\end{tabular} & & & & & & & & & & $\begin{array}{l}\text { Map associated pipes } \\
\text { and utilities and } \\
\text { unknown buried } \\
\text { structures. }\end{array}$ & & None (1) & \begin{tabular}{|l|} 
Mitchell, TH \\
and KA \\
Bergstrom
\end{tabular} & 1998 & $\mathrm{NA}$ & $\mathrm{NA}$ & \\
\hline $\begin{array}{l}\text { 116-C-5 Retention } \\
\text { Basin }\end{array}$ & $100-\mathrm{C}$ & & ? & ? & & & & & & & & & & $\begin{array}{l}\text { Locate and map } \\
\text { perimeter of basins, } \\
\text { and unknown features. } \\
\text { Determine optimum } \\
\text { locations for core } \\
\text { penetrometer } \\
\text { technology (CPT) and } \\
\text { test pits. }\end{array}$ & & BHI-00716 & \begin{tabular}{|l|} 
Bergstrom, \\
KA and TH \\
Mitchell
\end{tabular} & Feb-96 & 38 & Yes & \\
\hline $118-\mathrm{C}-1$ & $100-\mathrm{C}$ & $8-93$ & & & & & & & & & & & & burial ground & & $\begin{array}{l}\text { WHC-SD-EN- } \\
\text { DP-067 (1). } \\
\text { WHC-SD-EN- } \\
\text { TI-247 (2). }\end{array}$ & \begin{tabular}{|l|} 
1) Mitchell, \\
TH and KA \\
Bergstrom. \\
2) Mitchell \\
\end{tabular} & $\begin{array}{l}\text { 1) Feb-94. } \\
\text { 2) Sep-94 }\end{array}$ & $\begin{array}{l}\text { 1) } 85, \\
\text { 2) } 90\end{array}$ & $\begin{array}{l}\text { 1) No. } \\
\text { 2) Yes }\end{array}$ & \\
\hline $\begin{array}{l}\text { 100-D-32 Burial } \\
\text { Ground }\end{array}$ & $100-\mathrm{D}$ & & \begin{tabular}{|l|} 
GSSI SRI \\
$10 \mathrm{~A}$ with \\
$200 \mathrm{MHz}$ \\
antenna
\end{tabular} & $\begin{array}{l}\text { Geonics } \\
\text { EM-31D }\end{array}$ & & & & & & & & & & $\begin{array}{l}\text { Map western effluent } \\
\text { line and other } \\
\text { subsurface features. }\end{array}$ & & None (1) & \begin{tabular}{|l|} 
Mitchell, TH \\
and KA \\
Bergstrom
\end{tabular} & 1999 & $\mathrm{NA}$ & $\mathrm{NA}$ & \\
\hline $\begin{array}{l}\text { 100-D-6 Burial } \\
\text { Ground }\end{array}$ & 100-D & & \begin{tabular}{|l|} 
GSSI SRI \\
$10 \mathrm{~A}$ with \\
$200 \mathrm{MHz}$ \\
antenna \\
\end{tabular} & $\begin{array}{l}\text { Geonics } \\
\text { EM-31D }\end{array}$ & & & & & & & & & & $\begin{array}{l}\text { Map western effluent } \\
\text { line and other } \\
\text { subsurface features. }\end{array}$ & & None (1) & \begin{tabular}{|l|} 
Mitchell, TH \\
and KA \\
Bergstrom
\end{tabular} & 1999 & $\mathrm{NA}$ & $\mathrm{NA}$ & \\
\hline
\end{tabular}




\begin{tabular}{|c|c|c|c|c|c|c|c|c|c|c|c|c|c|c|c|c|c|c|c|c|c|}
\hline \multirow[b]{2}{*}{ Site/Location } & \multirow[b]{2}{*}{ Area } & \multirow[b]{2}{*}{\begin{tabular}{|c|} 
Date(s) \\
of Survey
\end{tabular}} & \multicolumn{11}{|c|}{ Geophysical Methods Used } & \multirow[b]{2}{*}{ Survey Objectives } & \multirow[b]{2}{*}{$\begin{array}{c}\text { Max. } \\
\text { Depth } \\
\text { (ft) }\end{array}$} & \multirow[b]{2}{*}{$\begin{array}{l}\text { Reference } \\
\text { (Doc. No.) }\end{array}$} & \multirow[b]{2}{*}{ Author(s) } & \multirow[b]{2}{*}{ Date } & \multicolumn{2}{|c|}{ Bibliography } & \multirow[b]{2}{*}{ Comments } \\
\hline & & & GPR & EM & IP & 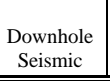 & $\begin{array}{c}\text { Seismic } \\
\text { (Acoustic) }\end{array}$ & Resistivity & ERT & Gravity & Magnetics & $\begin{array}{c}\text { Metal } \\
\text { Detector }\end{array}$ & Other & & & & & & \begin{tabular}{|c|}
$\begin{array}{c}\text { Ref. } \\
\text { Number }\end{array}$ \\
\end{tabular} & Abstract? & \\
\hline $\begin{array}{l}107-D-1 \text { Sludge } \\
\text { trench }\end{array}$ & 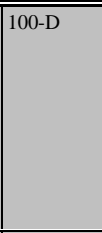 & & \begin{tabular}{|l|} 
GSSI SRI \\
System \\
$10 \mathrm{~A}$ with \\
$200-\mathrm{MHz}$ \\
antenna
\end{tabular} & $\begin{array}{l}\begin{array}{l}\text { Geonics } \\
\text { EM-31 }\end{array} \\
\end{array}$ & & & & & & & & & & \begin{tabular}{|l|} 
Locate, maa, and/or \\
verify locations of \\
subsurface pipelines, \\
utitities, sludge \\
disposal trenches, and \\
any unknown features \\
(e.e.g., major concen- \\
tration of debris, \\
pipelines, and utilities).
\end{tabular} & & $\begin{array}{l}\text { BHI-00786 } \\
\end{array}$ & \begin{tabular}{|l|} 
Bergstrom, \\
KA: \\
Mitchell, TH
\end{tabular} & May-96 & 40 & $\begin{array}{l}\text { Yes } \\
\end{array}$ & \\
\hline $\begin{array}{l}\text { 107-D-2 Sludge } \\
\text { trench }\end{array}$ & 100-D & & \begin{tabular}{|l|} 
GSSI SRI \\
SSstem \\
10A with \\
200-MHz \\
antenna \\
\end{tabular} & \begin{tabular}{|l|l} 
Geonics \\
EM-31
\end{tabular} & & & & & & & & & & \begin{tabular}{|l|} 
Locate, map, and/or \\
verify locations of \\
subsurface pipelines, \\
utilities, sludge \\
disposal trenches, and \\
any unknown features \\
(e.s., major concen- \\
tration of debris, \\
pipelines, and utilities).
\end{tabular} & & BHI-00786 & \begin{tabular}{|l|} 
Bergsstrom, \\
KA; \\
Mitchell, TH \\
\end{tabular} & May-96 & 40 & Yes & \\
\hline 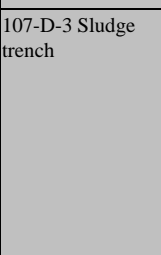 & 100-D & & \begin{tabular}{|l|} 
GSSI SRI \\
System \\
$10 \mathrm{~A}$ with \\
$200 \mathrm{MHz}$ \\
antenna \\
\end{tabular} & $\begin{array}{l}\begin{array}{l}\text { Geonics } \\
\text { EM-31 }\end{array} \\
\end{array}$ & & & & & & & & & & \begin{tabular}{|l|} 
Locate, map, and/or \\
verify locations of \\
subsurface pipelines, \\
utilities, sludge \\
disposat trenches, and \\
any unknown features \\
(e.g., major \\
concentration of \\
debris, pipelines, and \\
utilities).
\end{tabular} & & BHI-00786 & \begin{tabular}{|l|} 
Bergstrom, \\
KA: \\
Mitchell, TH
\end{tabular} & May-96 & 40 & Yes & \\
\hline $\begin{array}{l}\text { 107-D-4 Sludge } \\
\text { trench }\end{array}$ & 100-D & & \begin{tabular}{|l|} 
GSSI SRI \\
System \\
$10 \mathrm{~A}$ with \\
$200-\mathrm{MHz}$ \\
antenna \\
\end{tabular} & $\begin{array}{l}\text { Geonics } \\
\text { EM-31 }\end{array}$ & & & & & & & & & & \begin{tabular}{|l|} 
Locate, map, and/or \\
verify locations of \\
subsurface pipelines, \\
utitities, sludge \\
disposal trenches, and \\
any unknown features \\
(e.g., major concen- \\
tration of debris, \\
pipelines, and utilities).
\end{tabular} & & BHI-00786 & \begin{tabular}{|l|} 
Bergstrom, \\
KA: \\
Mitchell, TH
\end{tabular} & May-96 & 40 & Yes & \\
\hline $\begin{array}{l}\text { 107-D-5 Sludge } \\
\text { trench }\end{array}$ & 100-D & & \begin{tabular}{|l|} 
GSSI SRI \\
System \\
$10 \mathrm{~A}$ with \\
$200-\mathrm{MHz}$ \\
antenna \\
\end{tabular} & $\begin{array}{l}\text { Geonics } \\
\text { EM-31 }\end{array}$ & & & & & & & & & & \begin{tabular}{|l|} 
Locate, map, and/or \\
verify locations of \\
subsurface pipelines, \\
utilities, sludge \\
disposal trenches, and \\
any unknown features \\
(e.g., major concen- \\
tration of debris, \\
pipelines, and utilities).
\end{tabular} & & BHI-00786 & \begin{tabular}{|l|} 
Bergstrom, \\
KA: \\
Mitchell, TH
\end{tabular} & May-96 & 40 & Yes & \\
\hline 108-D & 100-D & $5-1-92$ & \begin{tabular}{|l|} 
GSSI SRI 8 \\
with 300 \\
MHz \\
antenna
\end{tabular} & & & & & & & & & & & \begin{tabular}{|l|} 
Map facilities, utilities, \\
and pipelines asso- \\
ciated with the former \\
108-D building.
\end{tabular} & & \begin{tabular}{l|} 
WHC-SD-EN- \\
TI-204, Rev. O
\end{tabular} & \begin{tabular}{|l|} 
Bergstrom, \\
$\mathrm{KA}$
\end{tabular} & 1994 & 31 & Yes. & \\
\hline 116-D-1A/1B & 100-D & \begin{tabular}{|l|}
$\begin{array}{l}9-27-91, \\
10-91, \\
12-11-2,\end{array}$ \\
2
\end{tabular} & \begin{tabular}{|l|} 
GSSI SRI 8 \\
with 100 \\
and 300 \\
MHz \\
antenna \\
\end{tabular} & & & & & & & & & & & $\begin{array}{l}\text { Locate trench. Find } \\
\text { decris-free zone for } \\
\text { emplacement of soil } \\
\text { gas probes. }\end{array}$ & & \begin{tabular}{|l|} 
WHC-SD-EN- \\
TI-204, Rev. O
\end{tabular} & \begin{tabular}{|l|} 
Bergstrom, \\
KA
\end{tabular} & 1994 & 31 & Yes. & \\
\hline
\end{tabular}




\begin{tabular}{|c|c|c|c|c|c|c|c|c|c|c|c|c|c|c|c|c|c|c|c|c|c|}
\hline \multirow[b]{2}{*}{ Site/Location } & \multirow[b]{2}{*}{ Area } & \multirow[b]{2}{*}{\begin{tabular}{|c|}
$\begin{array}{c}\text { Date(s) } \\
\text { of Survey }\end{array}$ \\
\end{tabular}} & \multicolumn{11}{|c|}{ Geophysical Methods Used } & \multirow[b]{2}{*}{ Survey Objectives } & \multirow[b]{2}{*}{\begin{tabular}{|c} 
Max. \\
Depth \\
(ft)
\end{tabular}} & \multirow[b]{2}{*}{$\begin{array}{l}\text { Reference } \\
\text { (Doc. No.) }\end{array}$} & \multirow[b]{2}{*}{ Author(s) } & \multirow[b]{2}{*}{ Date } & \multicolumn{2}{|c|}{ Bibliography } & \multirow[b]{2}{*}{ Comments } \\
\hline & & & GPR & EM & IP & $\begin{array}{c}\text { Downhole } \\
\text { Seismic }\end{array}$ & $\begin{array}{c}\text { Seismic } \\
\text { (Acoustic) }\end{array}$ & Resistivity & ERT & Gravity & Magnetics & $\begin{array}{l}\text { Metal } \\
\text { Detector }\end{array}$ & Other & & & & & & \begin{tabular}{|c} 
Ref. \\
Number
\end{tabular} & Abstract? & \\
\hline \begin{tabular}{|l|} 
116-D-1A/1B Liquid \\
Disposal Trench
\end{tabular} & 100-D & & \begin{tabular}{|l|} 
GSSI SIR 8 \\
and $10 \mathrm{~A}$ \\
with $300 \& 8$ \\
$100 \mathrm{MHz}$ \\
antenna
\end{tabular} & & & & & & & & & & & $\begin{array}{l}\begin{array}{l}\text { Delineate utilities and } \\
\text { subsurface features and } \\
\text { trench boundary. }\end{array} \\
\end{array}$ & $\overline{\mid 14-16^{\prime}}$ & BHI-00396 & \begin{tabular}{|l|l} 
Bergstrom, \\
KA, and TH \\
Mitchell
\end{tabular} & \begin{tabular}{|l|l|} 
Jan-96 \\
\end{tabular} & 36 & Yes & \\
\hline 116-D-2 Pluto Crib & 100-D & $1-7-92$ & \begin{tabular}{|l|} 
GSSI SRI 8 \\
with 100 \\
and 300 \\
$\mathrm{MHz}$ \\
antenna
\end{tabular} & & & & & & & & & & & \begin{tabular}{|l|} 
Verify proposed \\
borehole is located \\
within suspected Pluto \\
Crib. If not, locate the \\
Pluto Crib. \\
\end{tabular} & & \begin{tabular}{|l|} 
WHC-SD-EN- \\
TI-204, Rev. O
\end{tabular} & $\begin{array}{l}\text { Bergstrom, } \\
\text { KA }\end{array}$ & 1994 & 31 & Yes. & \\
\hline 116-D-2 Pluto Crib & 100-D & & \begin{tabular}{|l|} 
GSSI SIR 8 \\
and $10 \mathrm{~A}$ \\
with $300 \& \&$ \\
$100 \mathrm{MHz}$ \\
antenna \\
\end{tabular} & & & & & & & & & & & \begin{tabular}{|l|}
$\begin{array}{l}\text { Delineate utilities and } \\
\text { subsurface features, } \\
\text { locate and map septic } \\
\text { system and piping, and } \\
\text { locate crib. }\end{array}$ \\
\end{tabular} & $14-16^{\prime}$ & \begin{tabular}{|l|} 
ERC IOM \\
$\# 039006$
\end{tabular} & \begin{tabular}{|l|} 
Mitchell, TH \\
and KA \\
Bergstrom
\end{tabular} & 1996 & $\mathrm{NA}$ & $\mathrm{NA}$ & \\
\hline $\begin{array}{l}\text { 116-D-3 French } \\
\text { Drain }\end{array}$ & 100-D & 11-14-91 & \begin{tabular}{|l|} 
GSSI SRI 8 \\
with 100 \\
MHz \\
antenna
\end{tabular} & & & & & & & & & & & \begin{tabular}{|l|} 
Locate french drain \\
and map locations of \\
utilities, pipes, and \\
buildings that once \\
were located at the site.
\end{tabular} & & $\begin{array}{l}\text { WHC-SD-EN- } \\
\text { TI-204, Rev. O }\end{array}$ & \begin{tabular}{|l} 
Bergstrom, \\
KA
\end{tabular} & 1994 & 31 & Yes. & \\
\hline $\begin{array}{l}\text { 116-D-3 French } \\
\text { Drain }\end{array}$ & 100-D & & \begin{tabular}{|l|} 
GSSI SIR 8 \\
and $10 \mathrm{~A}$ \\
with $300 \mathrm{\&}$ \\
$100 \mathrm{MHz}$ \\
antenna
\end{tabular} & & & & & & & & & & & $\begin{array}{l}\text { Delineate area of } \\
\text { french drain and } \\
\text { associated piping. }\end{array}$ & $14-16^{\prime}$ & \begin{tabular}{|l} 
ERC IOM \\
$\# 039006$
\end{tabular} & \begin{tabular}{|l|} 
Mitchell, TH \\
and KA \\
Bergstrom
\end{tabular} & 1991,1996 & NA & $\mathrm{NA}$ & \\
\hline 116-D-4 & 100-D & 11-14-91 & \begin{tabular}{|l|} 
GSSI SRI 8 \\
with 100 \\
MHz \\
antenna
\end{tabular} & & & & & & & & & & & $\begin{array}{l}\text { Locate east-west } \\
\text { trending pipe that } \\
\text { could possibly lead } \\
\text { into trench. }\end{array}$ & & $\begin{array}{l}\text { WHC-SD-EN- } \\
\text { TI-204, Rev. O }\end{array}$ & \begin{tabular}{|l} 
Bergstrom, \\
KA
\end{tabular} & 1994 & 31 & Yes. & \\
\hline $\begin{array}{l}\text { 116-D-4 French } \\
\text { Drain }\end{array}$ & 100-D & & \begin{tabular}{|l|} 
GSSI SIR 8 \\
and $10 \mathrm{~A}$ \\
with $300 \& \&$ \\
$100 \mathrm{MHz}$ \\
antenna
\end{tabular} & & & & & & & & & & & $\begin{array}{l}\text { Delineate area of } \\
\text { french drain and } \\
\text { associated piping. }\end{array}$ & $14-16^{\prime}$ & $\begin{array}{l}\text { ERC IOM \# } \\
039006\end{array}$ & \begin{tabular}{|l|} 
Mitchell, TH \\
and KA \\
Bergstrom
\end{tabular} & 1991,1996 & $\mathrm{NA}$ & $\mathrm{NA}$ & \\
\hline 116-D-5 Outfall & 100-D & $12-5-91$ & \begin{tabular}{|l|} 
GSSI SRI 8 \\
with 300 \\
MHz \\
antenna \\
\end{tabular} & & & & & & & & & & & $\begin{array}{l}\text { Locate utilities and } \\
\text { buried debris that may } \\
\text { affect the drilling of a } \\
\text { borehole. }\end{array}$ & & \begin{tabular}{|l|} 
WHC-SD-EN- \\
TI-204, Rev. O
\end{tabular} & $\begin{array}{l}\text { Bergstrom, } \\
\text { KA }\end{array}$ & 1994 & 31 & Yes. & \\
\hline $\begin{array}{l}\text { 116-D-6 Cushion } \\
\text { Corridor }\end{array}$ & 100-D & $1-30-92$ & \begin{tabular}{|l|} 
GSSI SRI 8 \\
with 100 \\
MHz \\
antenna
\end{tabular} & & & & & & & & & & & $\begin{array}{l}\begin{array}{l}\text { Locate cushion } \\
\text { corridor. }\end{array} \\
\end{array}$ & & \begin{tabular}{|l|} 
WHC-SD-EN- \\
TI-204, Rev. O
\end{tabular} & \begin{tabular}{|l} 
Bergstrom, \\
KA
\end{tabular} & 1994 & 31 & Yes. & \\
\hline $\begin{array}{l}\text { 116-D-6 French } \\
\text { Drain }\end{array}$ & 100-D & & \begin{tabular}{|l|} 
GSSI SIR 8 \\
with 300 \\
MHz \\
antenna
\end{tabular} & & & & & & & & & & & $\begin{array}{l}\text { Delineate area of } \\
\text { french drain and } \\
\text { associated piping. }\end{array}$ & $14-16^{\prime}$ & $\begin{array}{l}\text { ERC IOM \# } \\
039006\end{array}$ & \begin{tabular}{|l|} 
Mitchell, TH \\
and KA \\
Bergstrom
\end{tabular} & 1992,1996 & $\mathrm{NA}$ & $\mathrm{NA}$ & \\
\hline $\begin{array}{l}\text { 116-D-7 Retention } \\
\text { Basin }\end{array}$ & 100-D & & \begin{tabular}{|l|} 
GSSI SRI \\
System \\
$10 \mathrm{~A}$ with \\
200-MHz \\
antenna \\
\end{tabular} & \begin{tabular}{|l} 
Geonics \\
EM-31
\end{tabular} & & & & & & & & & & \begin{tabular}{|l|} 
Locate, map, and/or \\
verify locations of \\
subsurface pipelines \\
and utilities and any \\
unknown features (e.g... \\
major concentration of \\
debris). \\
\end{tabular} & & BHI-00786 & \begin{tabular}{|l|} 
Bergstrom, \\
KA; \\
Mitchell, TH
\end{tabular} & May-96 & 40 & Yes & \\
\hline
\end{tabular}




\begin{tabular}{|c|c|c|c|c|c|c|c|c|c|c|c|c|c|c|c|c|c|c|c|c|c|}
\hline \multirow[b]{2}{*}{ Site/Location } & \multirow[b]{2}{*}{ Area } & \multirow[b]{2}{*}{\begin{tabular}{|c} 
Date(s) \\
of Survey
\end{tabular}} & \multicolumn{11}{|c|}{ Geophysical Methods Used } & \multirow[b]{2}{*}{ Survey Objectives } & \multirow[b]{2}{*}{$\begin{array}{c}\text { Max. } \\
\text { Depth } \\
\text { (fit) }\end{array}$} & \multirow[b]{2}{*}{$\begin{array}{l}\text { Reference } \\
\text { (Doc. No.) }\end{array}$} & \multirow[b]{2}{*}{ Author(s) } & \multirow[b]{2}{*}{ Date } & \multicolumn{2}{|c|}{ Bibliography } & \multirow[b]{2}{*}{ Comments } \\
\hline & & & GPR & $\mathrm{EM}$ & IP & $\begin{array}{c}\text { Downhole } \\
\text { Seismic }\end{array}$ & $\begin{array}{c}\text { Seismic } \\
\text { (Acoustic) }\end{array}$ & Resistivity & ERT & Gravity & Magnetics & \begin{tabular}{|c|} 
Metal \\
Detector \\
\end{tabular} & Other & & & & & & \begin{tabular}{|c|}
$\begin{array}{c}\text { Ref. } \\
\text { Number }\end{array}$ \\
\end{tabular} & Abstract? & \\
\hline $\begin{array}{l}116-\text {-D8 Cask } \\
\text { Storage Pad }\end{array}$ & 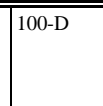 & 12-17-93 & \begin{tabular}{|l|l|} 
GSSI SIR 8 \\
with 300 \\
MHz \\
antenna
\end{tabular} & & & & & & & & & & & $\begin{array}{l}\begin{array}{l}\text { Locate french drain } \\
\text { associated with cask } \\
\text { storage pad. }\end{array} \\
\end{array}$ & $10-14^{\prime}$ & $\begin{array}{l}\text { WHC-SD-EN- } \\
\text { TI-224, IOM } \\
\# 039006\end{array}$ & \begin{tabular}{|l|} 
Mitchell, TH \\
and KA \\
Bergstrom
\end{tabular} & 1994 & $\mathrm{NA}$ & $\mathrm{NA}$ & \\
\hline 116-D-9 & 100-D & $\begin{array}{l}11-21-91, \\
2-12-92\end{array}$ & $\mid \begin{array}{l}\text { GSSI SRI I } \\
\text { with } 300 \\
\text { MHz } \\
\text { antenna }\end{array}$ & & & & & & & & & & & $\begin{array}{l}\text { Locate utilities and } \\
\text { buried debris. Locate } \\
\text { drilling site. }\end{array}$ & & $\begin{array}{l}\text { WHC-SD-EN- } \\
\text { TI-204, Rev. O }\end{array}$ & \begin{tabular}{|l|}
$\begin{array}{l}\text { Bergstrom, } \\
\text { KA }\end{array}$ \\
\end{tabular} & 1994 & 31 & Yes. & \\
\hline 116-D-9 Crib & 100-D & & \begin{tabular}{|l|} 
GSSI SIR 8 \\
and 10A \\
with 300 \\
MHz \\
antenna
\end{tabular} & & & & & & & & & & & \begin{tabular}{|l|} 
Delineate area of crib \\
and any underground \\
piping.
\end{tabular} & $\mid 14-16^{\prime}$ & \begin{tabular}{|l|} 
ERC IOM \\
$\# \# 39006$
\end{tabular} & \begin{tabular}{|l|} 
Mitchell, TH \\
and KA \\
Bergstrom
\end{tabular} & 1996 & $\mathrm{NA}$ & $\mathrm{NA}$ & \\
\hline \begin{tabular}{|l|l|}
$118-D-5$ Ball 3X \\
Burial Ground
\end{tabular} & 100-D & \begin{tabular}{|l|}
$2-29-93$, \\
$1-19-94$
\end{tabular} & \begin{tabular}{|l|} 
GSSI SIR 8 \\
with 300 \\
MHz \\
antenna
\end{tabular} & & & & & & & & & & & \begin{tabular}{|l} 
Locate trench. \\
\end{tabular} & $10-13 \mathrm{ft}$ & $\begin{array}{l}\text { WHC-SD-EN- } \\
\text { TI-226 }\end{array}$ & \begin{tabular}{|l|} 
Mitchell, TH \\
and KA \\
Bergstrom
\end{tabular} & May-94 & 88 & No & \\
\hline $130-\mathrm{D}-1$ & 100-D & $1-24-92$ & \begin{tabular}{|l|} 
GSSI SRI \\
with 300 \\
MHz \\
antenna
\end{tabular} & & & & & & & & & & & $\begin{array}{l}\text { Locate utilities and } \\
\text { buried debris. Locate } \\
\text { excavation boundary } \\
\text { for gas storage tank } \\
\text { that had been removed. }\end{array}$ & & $\begin{array}{l}\text { WHC-SD-EN- } \\
\text { TI-204, Rev. O }\end{array}$ & \begin{tabular}{|l} 
Bergstrom, \\
KA
\end{tabular} & 1994 & 31 & Yes. & \\
\hline 130-D-1 & 100-D & & $\mathrm{x}$ & & & & & & & & & $?$ & & $\begin{array}{l}\text { Locate and map } \\
\text { underground fuel } \\
\text { storage tanks, pipes, } \\
\text { and cables. }\end{array}$ & $15 \mathrm{ft}$ & \begin{tabular}{|l}
$\begin{array}{l}\text { PNL letter } \\
\text { report to Ron } \\
\text { Shuck, WHO }\end{array}$ \\
\end{tabular} & \begin{tabular}{|l|}
$\begin{array}{l}\text { G.A.A. } \\
\text { Sandness }\end{array}$ \\
\end{tabular} & 16-Aug-89 & $\mathrm{NA}$ & $\mathrm{NA}$ & \\
\hline $130-\mathrm{D}-2$ & 100-D & & $\mathrm{x}$ & & & & & & & & & $\mathrm{x}$ & & $\begin{array}{l}\text { Locate and map } \\
\text { underground fuel } \\
\text { storage tanks, pipes, } \\
\text { and cables. }\end{array}$ & $15 \mathrm{ft}$ & \begin{tabular}{|l|} 
PNLL letter \\
report to Mark \\
Morton, WHO
\end{tabular} & \begin{tabular}{|l|}
$\begin{array}{l}\text { G.A.A. } \\
\text { Sandness }\end{array}$ \\
\end{tabular} & 10-May-90 & $\mathrm{NA}$ & $\mathrm{NA}$ & \\
\hline \begin{tabular}{|l} 
132-D-3 Effluent \\
Pumping Station
\end{tabular} & 100-D & \begin{tabular}{|l|}
$1-7-92,2-9$ \\
$12-92$
\end{tabular} & 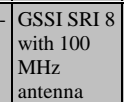 & & & & & & & & & & & $\begin{array}{l}\text { Locate utilities and } \\
\text { buried debris and site } \\
\text { free of drilling } \\
\text { bostructions. }\end{array}$ & & $\begin{array}{l}\text { WHC-SD-EN- } \\
\text { TI-204, Rev. O }\end{array}$ & $\begin{array}{l}\text { Bergstrom, } \\
\text { KA }\end{array}$ & 1994 & 31 & Yes. & \\
\hline \begin{tabular}{|l}
$1607-D 3$ Septic \\
System
\end{tabular} & 100-D & $12-17-93$ & \begin{tabular}{|l|} 
GSSI SIR 8 \\
with 300 \\
MHz \\
antenna
\end{tabular} & & & & & & & & & & & $\begin{array}{l}\begin{array}{l}\text { Locate septic tank and } \\
\text { drain field. }\end{array} \\
\end{array}$ & $\mid 10-15^{\prime}$ & $\begin{array}{l}\text { WHC-SD-EN- } \\
\text { TI-227 }\end{array}$ & \begin{tabular}{|l|} 
Bergstrom, \\
Mitithell, and \\
Szzwartz
\end{tabular} & Apr-94 & 25 & No. & \\
\hline \begin{tabular}{|l}
$1607-D 4$ Septic \\
System
\end{tabular} & 100-D & $\begin{array}{l}1-28-92, \\
2-11-92\end{array}$ & 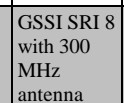 & & & & & & & & & & & \begin{tabular}{|l|} 
Locate sites for soil gas \\
probes that are free of \\
underground obstruc- \\
tions.
\end{tabular} & & $\begin{array}{l}\text { WHC-SD-EN- } \\
\text { TI-204, Rev. O }\end{array}$ & \begin{tabular}{|l} 
Bergstrom, \\
KA
\end{tabular} & 1994 & 31 & Yes. & \\
\hline \begin{tabular}{|l} 
1714-D Oil/Paint \\
Storage and 1715-D \\
Solvent Storage
\end{tabular} & 100-D & 5-27-92 & \begin{tabular}{|l|} 
GSSS SIR 8 \\
with 300 \\
MHz \\
antenna
\end{tabular} & & & & & & & & & & & $\begin{array}{l}\text { Locate foundation of } \\
\text { building and areas } \\
\text { where solvents, stet. } \\
\text { may have been } \\
\text { deposited }\end{array}$ & & $\begin{array}{l}\text { WHC-SD-EN- } \\
\text { TI-204, Rev. O }\end{array}$ & \begin{tabular}{|l}
$\begin{array}{l}\text { Bergstrom, } \\
\text { KA }\end{array}$ \\
\end{tabular} & 1994 & 31 & Yes. & \\
\hline \begin{tabular}{|l} 
190-D Sodium \\
Dichromate North \\
Transfer Line
\end{tabular} & 100-D & & \begin{tabular}{|l|} 
GSSI SIR \\
10A with \\
300 $\mathrm{MHz}$ \\
antenna \\
\end{tabular} & & & & & & & & & & & \begin{tabular}{|l} 
Locate and map \\
transfer line and \\
identify all bends, \\
encasements or other \\
potential weak spots.
\end{tabular} & & \begin{tabular}{|l} 
ERC IOM \\
\#051508
\end{tabular} & \begin{tabular}{|l|} 
Mitchell, TH \\
and KA \\
Bergstrom
\end{tabular} & 1997 & NA & NA & $\begin{array}{l}\text { Check } \\
\text { Sodium } \\
\text { Plume } \\
\text { Documents }\end{array}$ \\
\hline
\end{tabular}




\begin{tabular}{|c|c|c|c|c|c|c|c|c|c|c|c|c|c|c|c|c|c|c|c|c|c|}
\hline \multirow[b]{2}{*}{ Site/Location } & \multirow[b]{2}{*}{ Area } & \multirow[b]{2}{*}{$\begin{array}{c}\text { Date(s) } \\
\text { of Survey }\end{array}$} & \multicolumn{11}{|c|}{ Geophysical Methods Used } & \multirow[b]{2}{*}{ Survey Objectives } & \multirow[b]{2}{*}{\begin{tabular}{|c|}
$\begin{array}{c}\text { Max. } \\
\text { Depth } \\
\text { (ft) }\end{array}$ \\
\end{tabular}} & \multirow[b]{2}{*}{$\begin{array}{l}\text { Reference } \\
\text { (Doc. No.) } \\
\end{array}$} & \multirow[b]{2}{*}{ Author(s) } & \multirow[b]{2}{*}{ Date } & \multicolumn{2}{|c|}{ Bibliography } & \multirow[b]{2}{*}{ Comment: } \\
\hline & & & GPR & EM & IP & $\begin{array}{c}\text { Downhole } \\
\text { Seismic }\end{array}$ & $\begin{array}{c}\text { Seismic } \\
\text { (Acoustic) }\end{array}$ & Resistivity & ERT & Gravity & Magnetics & $\begin{array}{c}\text { Metal } \\
\text { Detector }\end{array}$ & Other & & & & & & \begin{tabular}{|c} 
Ref. \\
Number
\end{tabular} & Abstract? & \\
\hline D5-14 & 100-D & $1-3-92$ & \begin{tabular}{|l|} 
GSSI SRI 8 \\
with 100 \\
MHz \\
antenna
\end{tabular} & & & & & & & & & & & \begin{tabular}{l|} 
Locate utilities and \\
buried debris that may \\
affect the drilling of a \\
borehole.
\end{tabular} & & $\begin{array}{l}\text { WHC-SD-EN- } \\
\text { TI-204, Rev. O }\end{array}$ & $\begin{array}{l}\text { Bergstrom, } \\
\text { KA }\end{array}$ & 1994 & 31 & Yes. & \\
\hline D5-15 & 100-D & $1-3-92$ & \begin{tabular}{|l|} 
GSSI SRI 8 \\
with 100 \\
MHz \\
antenna \\
\end{tabular} & & & & & & & & & & & $\begin{array}{l}\text { Locate utilities and } \\
\text { buried debris that may } \\
\text { affect the drilling of a } \\
\text { borehole. }\end{array}$ & & $\begin{array}{l}\text { WHC-SD-EN- } \\
\text { TI-204, Rev. O }\end{array}$ & \begin{tabular}{|l} 
Bergstrom, \\
KA
\end{tabular} & 1994 & 31 & Yes. & \\
\hline D5-17 & 100-D & $1-3-92$ & \begin{tabular}{|l|} 
GSSI SRI 8 \\
with 100 \\
MHz \\
antenna \\
\end{tabular} & & & & & & & & & & & $\begin{array}{l}\text { Locate utilities and } \\
\text { buried debris that may } \\
\text { affect the drilling of a } \\
\text { borehole. }\end{array}$ & & $\begin{array}{l}\text { WHC-SD-EN- } \\
\text { TI-204, Rev. O }\end{array}$ & $\begin{array}{l}\text { Bergstrom, } \\
\text { KA }\end{array}$ & 1994 & 31 & Yes. & \\
\hline Paint Shop & 100-D & \begin{tabular}{|l|}
$4-15-92$, \\
$5-5-92$
\end{tabular} & \begin{tabular}{|l|} 
GSSI SRI \\
with 300 \\
MHz \\
antenna
\end{tabular} & & & & & & & & & & & \begin{tabular}{|l|} 
Locate buried remains \\
of the paint solvent \\
buildings and identify \\
any excavations around \\
the shop that may have \\
been used for disposal.
\end{tabular} & $8-12$ & $\begin{array}{l}\text { WHC-SD-EN- } \\
\text { TI-204, Rev. O }\end{array}$ & \begin{tabular}{|l} 
Bergstrom, \\
KA
\end{tabular} & 1994 & 31 & Yes. & \\
\hline Salt Dissolving Pit & 100-D & $5-27-92$ & \begin{tabular}{|l|} 
GSSI SRI 8 \\
with 300 \\
MHz \\
antenna \\
\end{tabular} & & & & & & & & & & & $\begin{array}{l}\begin{array}{l}\text { Locate salt-dissolving } \\
\text { pit. }\end{array} \\
\end{array}$ & $10-12$ & $\begin{array}{l}\text { WHC-SD-EN- } \\
\text { TI-204, Rev. O }\end{array}$ & $\begin{array}{l}\text { Bergstrom, } \\
\text { KA }\end{array}$ & 1994 & 31 & Yes. & \\
\hline \begin{tabular}{|l} 
Sodium Dichromate \\
Pipeline from \\
Transfer Station to \\
183-DR \\
\end{tabular} & 100-D & & \begin{tabular}{|l|} 
GSSI SIR \\
$10 \mathrm{~A}$ with \\
$300 \mathrm{MHz}$ \\
antenna \\
\end{tabular} & & & & & & & & & & & $\begin{array}{l}\text { Locate pipeline and } \\
\text { other utilities and } \\
\text { structures. }\end{array}$ & $9-12^{\prime}$ & None (1) & \begin{tabular}{|l|} 
Bergstrom, \\
KA, and TH \\
Mitchell
\end{tabular} & 1999 & NA & NA & \\
\hline $\begin{array}{l}\text { Sodium Dichromate } \\
\text { Transfer Station }\end{array}$ & 100-D & 6-16-93 & \begin{tabular}{|l|} 
GSSI SIR 8 \\
with 300 \\
MHz \\
antenna
\end{tabular} & & & & & & & & & & & \begin{tabular}{|l|} 
Locate subsurface \\
pipes, utilities, and \\
underground structures \\
related to transfer \\
station.
\end{tabular} & $10-15^{\prime}$ & $\begin{array}{l}\text { WHC-SD-EN- } \\
\text { TI-178 }\end{array}$ & $\begin{array}{l}\text { Bergstrom, } \\
\text { KA, and TH } \\
\text { Mitchell }\end{array}$ & Oct-93 & 18 & No. & \\
\hline $\begin{array}{l}\text { TP\&L Trailer } \\
\text { Ground Rods }\end{array}$ & 100-D & & \begin{tabular}{|l} 
GSSI SIR \\
$10 \mathrm{~A}$ with \\
$300 \mathrm{MHz}$ \\
antenna \\
\end{tabular} & & & & & & & & & & & $\begin{array}{l}\text { Map utilities, pipelines, } \\
\text { and other subsurface } \\
\text { features. }\end{array}$ & & None (1) & $\begin{array}{l}\text { Mitchell, TH } \\
\text { and KA } \\
\text { Bergstrom }\end{array}$ & 1998 & NA & $\mathrm{NA}$ & \\
\hline 116-DR-1/1 16-DR-2 & 100-DR & $\mid 10-25-91$ & \begin{tabular}{|l|} 
GSSI SRI 8 \\
with 100 \\
MHz \\
antenna \\
\end{tabular} & & & & & & & & & & & $\begin{array}{l}\text { Locate two sites for } \\
\text { proposed boreholes } \\
\text { free of buried } \\
\text { obstructions. }\end{array}$ & & $\begin{array}{l}\text { WHC-SD-EN- } \\
\text { TI-204, Rev. O }\end{array}$ & $\begin{array}{l}\text { Bergstrom, } \\
\text { KA }\end{array}$ & 1994 & 31 & Yes. & \\
\hline \begin{tabular}{|l} 
116-DR-1 Liquid \\
Effluent Trench
\end{tabular} & 100-DR & & $\begin{array}{l}\text { GSSI SRI } \\
\text { System } \\
10 \mathrm{~A} \text { with } \\
\text { 200-MHz } \\
\text { antenna }\end{array}$ & $\begin{array}{l}\text { Geonics } \\
\text { EM-31 }\end{array}$ & & & & & & & & & & $\begin{array}{l}\text { Map the extent of } \\
\text { liquid disposal trenches } \\
\text { and locate and } \\
\text { accurately map any } \\
\text { unknown features (e.g., } \\
\text { major concentration of } \\
\text { debris, pipelines, and } \\
\text { utilities). }\end{array}$ & & BHI-00786 & \begin{tabular}{|l} 
Bergstrom, \\
KA; \\
Mitchell, TH
\end{tabular} & May-96 & 40 & Yes & \\
\hline $\begin{array}{l}\text { 116-DR-2 Liquid } \\
\text { Effluent Trench }\end{array}$ & 100-DR & & \begin{tabular}{|l} 
GSSI SRI \\
System \\
$10 \mathrm{~A}$ with \\
$200-\mathrm{MHz}$ \\
antenna
\end{tabular} & \begin{tabular}{|l} 
Geonics \\
EM-31
\end{tabular} & & & & & & & & & & $\begin{array}{l}\text { Map the extent of } \\
\text { liquid disposal trenches } \\
\text { and locate and accu- } \\
\text { rately map any } \\
\text { unknown features (e.g.., } \\
\text { major concentration of } \\
\text { debris, pipelines, and } \\
\text { utilities). }\end{array}$ & & BHI-00786 & \begin{tabular}{|l|} 
Bergstrom, \\
KA; \\
Mitchell, TH
\end{tabular} & May-96 & 40 & Yes & \\
\hline
\end{tabular}




\begin{tabular}{|c|c|c|c|c|c|c|c|c|c|c|c|c|c|c|c|c|c|c|c|c|c|}
\hline \multirow[b]{2}{*}{ Site/Location } & \multirow[b]{2}{*}{ Area } & \multirow[b]{2}{*}{\begin{tabular}{|c}
$\begin{array}{c}\text { Date(s) } \\
\text { of Survey }\end{array}$ \\
(a)
\end{tabular}} & \multicolumn{11}{|c|}{ Geophysical Methods Used } & \multirow[b]{2}{*}{ Survey Objectives } & \multirow[b]{2}{*}{$\mid \begin{array}{c}\text { Max. } \\
\text { Depth } \\
\text { (ft) }\end{array}$} & \multirow[b]{2}{*}{$\begin{array}{l}\text { Reference } \\
\text { (Doc. No.) }\end{array}$} & \multirow[b]{2}{*}{ Author(s) } & \multirow[b]{2}{*}{ Date } & \multicolumn{2}{|c|}{\begin{tabular}{c|} 
Bibliography \\
\end{tabular}} & \multirow[b]{2}{*}{ Comments } \\
\hline & & & \begin{tabular}{|l|l} 
GPR \\
\end{tabular} & EM & IP & \begin{tabular}{|c|}
$\begin{array}{c}\text { Downhole } \\
\text { Seismic }\end{array}$ \\
\end{tabular} & \begin{tabular}{|c} 
Seismic \\
(Acoustic)
\end{tabular} & Resistivity & ERT & Gravity & Magnetics & \begin{tabular}{|c|c} 
Metal \\
Detector \\
\end{tabular} & Other & & & & & & $\begin{array}{c}\text { Ref. } \\
\text { Number } \\
\end{array}$ & Abstract? & \\
\hline $\begin{array}{l}\begin{array}{l}116-\text {-DR-3 Storage } \\
\text { Basin }\end{array} \\
\end{array}$ & 1100-DR & $\begin{array}{l}6-14-93, \\
6-28-93, \\
8-17-93\end{array}$ & \begin{tabular}{|l|} 
GSSI SIR 8 \\
with 300 \\
MHz \\
antenna
\end{tabular} & & & & & & & & & & & $\begin{array}{l}\text { Locate boundaries. } \\
\text {. }\end{array}$ & 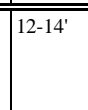 & $\begin{array}{l}\text { WHC-SD-EN- } \\
\text { TI-177 }\end{array}$ & \begin{tabular}{|l|l|} 
Mitchell, \\
and KA \\
Bergstrom
\end{tabular} & $\overline{\text { Nov-93 }}$ & 81 & No. & \\
\hline 116-DR-4 Pluto Crib & b 100-DR & 6-16-93 & \begin{tabular}{|l|} 
GSSS SIR 8 \\
with 300 \\
MHz \\
antenna \\
\end{tabular} & & & & & & & & & & & $\begin{array}{l}\text { Verify the location of } \\
\text { the 105-DR Pluto Crib, } \\
\text { 116-DR-4. }\end{array}$ & $\mid 12-14^{\prime}$ & $\begin{array}{l}\text { WHC-SD-EN- } \\
\text { TI-195 }\end{array}$ & \begin{tabular}{|l|} 
Bergstrom, \\
KA, and TH \\
Mitchell
\end{tabular} & 1993 & 20 & Yes & \\
\hline 116-DR-5 Outfall & 100-DR & $12-5-91$ & \begin{tabular}{|l|} 
GSSS SRI 8 \\
with 300 \\
MHz \\
antenna
\end{tabular} & & & & & & & & & & & $\begin{array}{l}\text { Locate utilities and } \\
\text { buried debris that may } \\
\text { affect the drilling of a } \\
\text { borehole. }\end{array}$ & & $\begin{array}{l}\text { WHC-SD-EN- } \\
\text { TI-204, Rev. O }\end{array}$ & \begin{tabular}{|l}
$\begin{array}{l}\text { Bergstrom, } \\
\text { KA }\end{array}$ \\
\end{tabular} & 1994 & 31 & 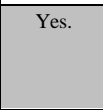 & \\
\hline $\begin{array}{l}\text { 116-DR-6 Liquid } \\
\text { Waste Disposal } \\
\text { Trench }\end{array}$ & $100-\mathrm{DR}$ & $\begin{array}{l}\begin{array}{l}12-15-93, \\
1-19-94\end{array} \\
1\end{array}$ & \begin{tabular}{|l|} 
GSSS SIR 8 \\
with 300 \\
MHz \\
antenna
\end{tabular} & & & & & & & & & & & \begin{tabular}{|l|}
$\begin{array}{l}\text { Locate the 116-DR-6 } \\
\text { trench. }\end{array}$ \\
\end{tabular} & $10-15^{\prime}$ & \begin{tabular}{|l} 
WHC-SD-EN- \\
T1-222
\end{tabular} & \begin{tabular}{|l|} 
Bergstrom, \\
KA
\end{tabular} & Aug-96 & 41 & Yes. & \\
\hline $\begin{array}{l}\text { 116-DR-7 Inkwell } \\
\text { Crib }\end{array}$ & 100-DR & 1996 & \begin{tabular}{|l|} 
GSSI SRI \\
$10 \mathrm{~A}$ with \\
$500 \mathrm{MHz}$ \\
antenna
\end{tabular} & & & & & & & & & & & Verify location of crib. & & $\begin{array}{l}\text { ERC IOM } \\
\# 039006\end{array}$ & \begin{tabular}{|l|} 
Mitchell, \\
and KA \\
Bergstrom
\end{tabular} & Jun-09 & $\mathrm{NA}$ & $\mathrm{NA}$ & \\
\hline \begin{tabular}{|l|}
$\begin{array}{l}\text { l16-DR-7 Inkwell } \\
\text { Crib }\end{array}$ \\
\end{tabular} & 100-DR & 6-16-93 & \begin{tabular}{|l|} 
GSSI SIR 8 \\
with 300 \\
MHz \\
antenna
\end{tabular} & & & & & & & & & & & Verify location of crib. & & $\begin{array}{l}\text { WHC-SD-EN- } \\
\text { TI-179 }\end{array}$ & \begin{tabular}{|l|}
$\begin{array}{l}\text { Mitchell, } \\
\text { and KA } \\
\text { Bergstrom }\end{array}$ \\
\end{tabular} & Jan-94 & 83 & No. & \\
\hline 116-DR-8 Crib & 100-DR & $12-15-93$ & \begin{tabular}{|l|} 
GSSI SIR 8 \\
with 300 \\
MHz \\
antenna
\end{tabular} & & & & & & & & & & & Locate edges of crib. & $10-15^{\prime}$ & $\begin{array}{l}\text { WHC-SD-EN- } \\
\text { TI-225 }\end{array}$ & \begin{tabular}{|l|}
$\begin{array}{l}\text { Kunk, JA } \\
\text { and } \\
\text { Bergstrom, } \\
\text { KA }\end{array}$ \\
\end{tabular} & Mar-94 & 65 & No. & \\
\hline $\begin{array}{l}\text { 116-DR-9 Retention } \\
\text { Basin }\end{array}$ & 100-DR & & \begin{tabular}{|l|} 
GSSI SRI \\
System \\
10A with \\
200-MHz \\
antenna \\
\end{tabular} & $\begin{array}{l}\text { Geonics } \\
\text { EM-31 }\end{array}$ & & & & & & & & & & $\begin{array}{l}\text { Locate, map, and/ or } \\
\text { verify locations of } \\
\text { subsurface pipelines } \\
\text { and utilities and any } \\
\text { unknown features (e.g., } \\
\text { major concentration of } \\
\text { debris). }\end{array}$ & & BHI-00786 & \begin{tabular}{|l|} 
Bergstrom, \\
KAst \\
Mitchell, TH
\end{tabular} & May-96 & 40 & Yes & \\
\hline $\begin{array}{l}\text { 132-DR-1 Waste } \\
\text { Water Pumping } \\
\text { Station }\end{array}$ & 100-DR & $12-29-93$ & \begin{tabular}{|l|} 
GSSI SIR 8 \\
with 300 \\
MHz \\
antenna
\end{tabular} & & & & & & & & & & & \begin{tabular}{|l|} 
Locate 132-DR-1 \\
waste water pump \\
station and associated \\
piping.
\end{tabular} & $10-15^{\prime}$ & $\begin{array}{l}\text { WHC-SD-EN- } \\
\text { TI-222 }\end{array}$ & $\begin{array}{l}\text { Bergstrom, } \\
\text { KA }\end{array}$ & Aug-96 & 41 & Yes & \\
\hline \begin{tabular}{|l|} 
183-DR Sodium \\
Dichromate Pipeline
\end{tabular} & 100-DR & & & & & & & & & & & & & & & None (1) & \begin{tabular}{|l|} 
Mitchell, TH \\
and KA \\
Bergstrom
\end{tabular} & 1999 & NA & NA & \begin{tabular}{|l|} 
Check \\
Sodium \\
Plume \\
Documents
\end{tabular} \\
\hline MO-980 Trailer Site & 100-DR & & \begin{tabular}{|l|} 
GSSI SIR \\
100 with \\
$300 \mathrm{MHz}$ \\
antenna
\end{tabular} & & & & & & & & & & & \begin{tabular}{|l} 
Locate subsurface \\
utilities and other \\
buried objects/debris.
\end{tabular} & $0-14^{\prime}$ & None (1) & \begin{tabular}{|l|} 
Mitchell, $\mathrm{TH}$ \\
and KA \\
Bergstrom
\end{tabular} & 1988 & NA & $\mathrm{NA}$ & \\
\hline 100-F-1 Depression & $100-\mathrm{F}$ & & \begin{tabular}{|l|} 
GSSI SIR \\
10 with \\
$300 \mathrm{MHz}$ \\
antenna
\end{tabular} & $\begin{array}{l}\text { Geonics } \\
\text { EM-31D }\end{array}$ & & & & & & & & & & $\begin{array}{l}\text { Determine whether } \\
\text { there is a subusuface } \\
\text { structure associated } \\
\text { with the depression. }\end{array}$ & $\mid 10-18^{\prime}$ & $\begin{array}{l}\text { BHI-00343 } \\
\text { Rev. 00 }\end{array}$ & \begin{tabular}{|l|} 
Bergstrom, \\
KA and TH \\
Mitchell
\end{tabular} & Jul-95 & 35 & No & \\
\hline
\end{tabular}




\begin{tabular}{|c|c|c|c|c|c|c|c|c|c|c|c|c|c|c|c|c|c|c|c|c|c|}
\hline \multirow[b]{2}{*}{ Site/Location } & \multirow[b]{2}{*}{ Area } & \multirow[b]{2}{*}{\begin{tabular}{|c|}
$\begin{array}{c}\text { Date(s) } \\
\text { of Survey }\end{array}$ \\
\end{tabular}} & \multicolumn{11}{|c|}{ Geophysical Methods Used } & \multirow[b]{2}{*}{ Survey Objectives } & \multirow[b]{2}{*}{\begin{tabular}{|c} 
Max. \\
Depth \\
$(\mathrm{ft})$
\end{tabular}} & \multirow[b]{2}{*}{$\begin{array}{l}\text { Reference } \\
\text { (Doc. No.) }\end{array}$} & \multirow[b]{2}{*}{ Author(s) } & \multirow[b]{2}{*}{ Date } & \multicolumn{2}{|c|}{ Bibliography } & \multirow[b]{2}{*}{ Comments } \\
\hline & & & GPR & EM & IP & $\begin{array}{c}\text { Downhole } \\
\text { Seismic }\end{array}$ & $\begin{array}{c}\text { Seismic } \\
\text { (Acoustic) }\end{array}$ & Resistivity & ERT & Gravity & Magnetics & $\begin{array}{c}\text { Metal } \\
\text { Detector }\end{array}$ & Other & & & & & & \begin{tabular}{|c} 
Ref. \\
Number
\end{tabular} & Abstract? & \\
\hline 100-F-14 Vent Pipe & $100-\mathrm{F}$ & & \begin{tabular}{|l|} 
GSSI SIR \\
10 with \\
$300 \mathrm{MHz}$ \\
antenna
\end{tabular} & $\begin{array}{l}\text { Geonics } \\
\text { EM-31D }\end{array}$ & & & & & & & & & & $\begin{array}{l}\text { Determine whether } \\
\text { there is a subsurface } \\
\text { structure beneath the } \\
\text { vent pipe and map } \\
\text { associated pipes. } \\
\end{array}$ & $10-18^{\prime}$ & $\begin{array}{l}\text { BHI-00343 } \\
\text { Rev. 00 }\end{array}$ & \begin{tabular}{|l|} 
Bergstrom, \\
KA, and TH \\
Mitchell
\end{tabular} & Jul-95 & 35 & No & \\
\hline 108-F Building & $100-\mathrm{F}$ & & & & & & & & & & & & & & & None (1) & \begin{tabular}{|l|}
$\begin{array}{l}\text { Mitchell, TH } \\
\text { and KA } \\
\text { Bergstrom }\end{array}$ \\
\end{tabular} & 1999 & $\mathrm{NA}$ & NA & \\
\hline $116-\mathrm{F}-14$ & $100-\mathrm{F}$ & $1-30-93$ & $\mathrm{x}$ & & & & & & & & & & & borehole siting & & $\begin{array}{l}\text { WHC-SD-EN- } \\
\text { TI-126 }\end{array}$ & \begin{tabular}{|l|}
$\begin{array}{l}\text { Mitchell, TH } \\
\text { and KA } \\
\text { Bergstrom }\end{array}$ \\
\end{tabular} & May-93 & 78 & No & \\
\hline $116-\mathrm{F}-2$ & $100-\mathrm{F}$ & $1-6-93$ & $\mathrm{x}$ & & & & & & & & & & & borehole siting & & $\begin{array}{l}\text { WHC-SD-EN- } \\
\text { TI-114 }\end{array}$ & \begin{tabular}{|l} 
Mitchell, \\
TH, Kunk, \\
and KA \\
Bergstrom \\
\end{tabular} & Feb-93 & 76 & no & \\
\hline $\begin{array}{l}116-\mathrm{F}-3 \text { Fuel } \\
\text { Storage Basin } \\
\text { Trench }\end{array}$ & $100-\mathrm{F}$ & $1 / 12 / 93$ & \begin{tabular}{|l|} 
GSSI SIR 8 \\
with 300 \\
MHz \\
antenna
\end{tabular} & \begin{tabular}{|l} 
Geonics \\
EM-31
\end{tabular} & & & & & & & & & & $\begin{array}{l}\text { Verify location of fuel } \\
\text { storage basin trench } \\
\text { and identify subsurface } \\
\text { obstructions within } \\
\text { trench. }\end{array}$ & $0-15^{\prime}$ & $\begin{array}{l}\text { WHC-SD-EN- } \\
\text { TI-118 (1). } \\
\text { WHC-SA- } \\
\text { 2018/CONF- } \\
\text { 9310160-1 (2) }\end{array}$ & \begin{tabular}{|l|} 
1) Bergstrom \\
and Mitchell. \\
2) \\
Bergstrom, \\
KA; \\
Mitchell, \\
TH; Kunk, \\
JR \\
\end{tabular} & $\begin{array}{l}\text { 1) Jul-93. } \\
\text { 2) Jul-93 }\end{array}$ & $\begin{array}{l}\text { 1) } 13 \\
\text { 2) } 12\end{array}$ & $\begin{array}{l}\text { 1) No. } \\
\text { 2) Yes. }\end{array}$ & \\
\hline 116-F-4 Pluto Crib & 100-F & $\begin{array}{l}3-9-93 \\
1 / 12 / 93 \\
1-22-93, \\
3-17-93\end{array}$ & \begin{tabular}{|l|} 
GSSI SIR 8 \\
with 300 \\
MHz \\
antenna
\end{tabular} & $\begin{array}{l}\text { Geonics } \\
\text { EM-31 }\end{array}$ & & & & & & & & & & 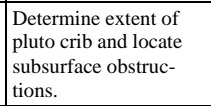 & $0-8^{\prime}$ & $\begin{array}{l}\text { WHC-SD-EN- } \\
\text { TI-119 }\end{array}$ & \begin{tabular}{|l|} 
Bergstrom, \\
KA, and TH \\
Mitchell
\end{tabular} & Jun-93 & 10 & No. & \\
\hline $\begin{array}{l}\text { 116-F-5 Ball Washer } \\
\text { Crib }\end{array}$ & 100-F & & \begin{tabular}{|l|} 
GSSI SIR \\
$10 \mathrm{~A}$ with \\
$300 \mathrm{MHz}$ \\
antenna \\
\end{tabular} & & & & & & & & & & & $\begin{array}{l}\text { Confirm location and } \\
\text { map boundaries. }\end{array}$ & $0-12^{\prime}$ & $\begin{array}{l}\text { ERC IOM } \\
\# 051553\end{array}$ & \begin{tabular}{|l|} 
Bergstrom, \\
KA, and TH \\
Mitchell \\
\end{tabular} & 1997 & NA & NA & \\
\hline $116-\mathrm{F}-6$ & 100-F & $1 / 6 / 93$ & $x$ & & & & & & & & & & & & & $\begin{array}{l}\text { WHC-SD-EN- } \\
\text { TI-115 (1). } \\
\text { WHC-SD-EN- } \\
\text { TI-247 (2) }\end{array}$ & \begin{tabular}{|l|} 
1) \\
Bergstrom, \\
KA, and TH \\
Mitchell. \\
2) Mitchell \\
\end{tabular} & $\begin{array}{l}\text { 1) Jan- }-93 \text {. } \\
\text { 2) Sep-94 }\end{array}$ & $\begin{array}{l}\text { 1) } 5 . \\
\text { 2) } 90\end{array}$ & $\begin{array}{l}\text { 1) No. } \\
\text { 2) Yes. }\end{array}$ & \\
\hline 116-F-9D & 100-F & $3-17-93$ & $\mathrm{x}$ & & & & & & & & & & & borehole siting & & $\begin{array}{l}\text { WHC-SD-EN- } \\
\text { TI-129 }\end{array}$ & \begin{tabular}{|l|} 
Bergstrom, \\
KA, and TH \\
Mitchell
\end{tabular} & Jun-93 & 9 & No & \\
\hline 199-F5-45 & 100-F & $6-30-92$ & \begin{tabular}{|l|} 
GSSI SRI 8 \\
with 300 \\
MHz \\
antenna
\end{tabular} & & & & & & & & & & & $\begin{array}{l}\text { Locate possible } \\
\text { underground utilities } \\
\text { and subsurface debris } \\
\text { that could be detri- } \\
\text { mental to drilling. }\end{array}$ & & $\begin{array}{l}\text { WHC-SD-EN- } \\
\text { TI-204 (1). } \\
\text { WHC-SA- } \\
\text { 2018/CONF- } \\
9310160-1 \text { (2) }\end{array}$ & \begin{tabular}{|l|} 
1) \\
Bergstrom, \\
KA. 2) \\
Bergstrom, \\
KA; \\
Mitchell, TH \\
and Kunk, \\
JR \\
\end{tabular} & $\begin{array}{l}\text { 1994. July } \\
\text { 1993. }\end{array}$ & $\begin{array}{l}\text { 1) } 31 . \\
\text { 2) } 12 \text {. }\end{array}$ & $\begin{array}{l}\text { 1) Yes. } \\
\text { 2) Yes. }\end{array}$ & \\
\hline 199-F5-46 & 100-F & $8-6-92$ & \begin{tabular}{|l|} 
GSSI SRI 8 \\
with 300 \\
MHz \\
antenna
\end{tabular} & & & & & & & & & & & $\begin{array}{l}\text { Locate possible } \\
\text { underground utilities } \\
\text { and subsurface debris } \\
\text { that could be } \\
\text { detrimental to drilling. }\end{array}$ & & $\begin{array}{l}\text { WHC-SD-EN- } \\
\text { TI-204, Rev. O }\end{array}$ & \begin{tabular}{|l} 
Bergstrom, \\
KA
\end{tabular} & 1994 & 31 & Yes. & \\
\hline
\end{tabular}




\begin{tabular}{|c|c|c|c|c|c|c|c|c|c|c|c|c|c|c|c|c|c|c|c|c|c|}
\hline \multirow{2}{*}{ Site/Location } & \multirow[b]{2}{*}{ Area } & \multirow[b]{2}{*}{$\begin{array}{c}\text { Date(s) } \\
\text { of Survey }\end{array}$} & \multicolumn{11}{|c|}{ Geophysical Methods Used } & \multirow[b]{2}{*}{ Survey Objectives } & \multirow[b]{2}{*}{\begin{tabular}{|c|} 
Max. \\
Depth \\
(ft) \\
\end{tabular}} & \multirow[b]{2}{*}{$\begin{array}{l}\text { Reference } \\
\text { (Doc. No.) }\end{array}$} & \multirow[b]{2}{*}{ Author(s) } & \multirow[b]{2}{*}{ Date } & \multicolumn{2}{|c|}{ Bibliography } & \multirow[b]{2}{*}{ Comments } \\
\hline & & & GPR & EM & IP & \begin{tabular}{|c|} 
Downhole \\
Seismic
\end{tabular} & $\begin{array}{c}\text { Seismic } \\
\text { (Acoustic) }\end{array}$ & Resistivity & ERT & Gravity & Magnetics & $\begin{array}{c}\text { Metal } \\
\text { Detector }\end{array}$ & Other & & & & & & $\begin{array}{c}\text { Ref. } \\
\text { Number }\end{array}$ & Abstract? & \\
\hline $199-\mathrm{F} 5-47$ & $100-\mathrm{F}$ & \begin{tabular}{|l|}
$6-30-92$, \\
$8-6-92$
\end{tabular} & \begin{tabular}{|l|} 
GSSI SRI 8 \\
with 300 \\
MHz \\
antenna
\end{tabular} & & & & & & & & & & & $\begin{array}{l}\text { Locate possible under- } \\
\text { ground utilities. Inves- } \\
\text { tigate the proposed } \\
\text { drill site for subsurface } \\
\text { anomalies that would } \\
\text { be detrimental to the } \\
\text { well. }\end{array}$ & & $\begin{array}{l}\text { WHC-SD-EN- } \\
\text { TI-204, Rev. O }\end{array}$ & \begin{tabular}{|l} 
Bergstrom, \\
KA
\end{tabular} & 1994 & 31 & $\begin{array}{l}\text { Yes } \\
\end{array}$ & \\
\hline 199-F5-48 & $100-\mathrm{F}$ & 6-30-92 & $\begin{array}{l}\text { GSSI SRI } 8 \\
\text { with 300 } \\
\text { MHz } \\
\text { antenna }\end{array}$ & & & & & & & & & & & $\begin{array}{l}\text { Locate possible under- } \\
\text { ground utilities. Inves- } \\
\text { tigate the proposed } \\
\text { drill site for subsurface } \\
\text { anomalies that would } \\
\text { be detrimental to the } \\
\text { well. }\end{array}$ & & $\begin{array}{l}\text { WHC-SD-EN- } \\
\text { TI-204, Rev. O }\end{array}$ & $\begin{array}{l}\text { Bergstrom, } \\
\text { KA }\end{array}$ & 1994 & 31 & Yes & \\
\hline PNL Parallel Pits & $100-\mathrm{F}$ & & \begin{tabular}{|l|} 
GSSI SIR \\
10 with \\
$300 \mathrm{MHz}$ \\
antenna \\
\end{tabular} & $\begin{array}{l}\text { Geonics } \\
\text { EM-31D }\end{array}$ & & & & & & & & & & \begin{tabular}{|l|}
$\begin{array}{l}\text { Locate and map the } \\
\text { pits, buried debris, and } \\
\text { trenches. }\end{array}$ \\
\end{tabular} & $10-18^{\prime}$ & $\begin{array}{l}\text { BHI-00343 } \\
\text { Rev. 00 }\end{array}$ & \begin{tabular}{|l|} 
Bergstrom, \\
KA, and TH \\
Mitchell
\end{tabular} & Jul-95 & 9 & No & \\
\hline $\begin{array}{l}\text { 100-H-5 Disposal } \\
\text { Site }\end{array}$ & $100-\mathrm{H}$ & & \begin{tabular}{|l|} 
GSSI SIR \\
$10 \mathrm{~A}$ with \\
$300 \mathrm{MHz}$ \\
antenna
\end{tabular} & & & & & & & & & & & $\begin{array}{l}\text { Locate and map trench } \\
\text { and associated } \\
\text { features. }\end{array}$ & & $\begin{array}{l}\text { ERC IOM } \\
\# 051553\end{array}$ & \begin{tabular}{|l|} 
Mitchell, TH \\
and KA \\
Bergstrom
\end{tabular} & 1997 & $\mathrm{NA}$ & $\mathrm{NA}$ & \\
\hline $\begin{array}{l}\text { 100-H-5 Sludge } \\
\text { Trench }\end{array}$ & $100-\mathrm{H}$ & & \begin{tabular}{|l|} 
GSSI SIR \\
$10 \mathrm{~A}$ with \\
$300 \mathrm{MHz}$ \\
antenna
\end{tabular} & \begin{tabular}{|l|} 
Geonics \\
EM-31D
\end{tabular} & & & & & & & & & & & & \begin{tabular}{|l} 
ERC IOM \\
$\# 051553$
\end{tabular} & \begin{tabular}{|l|} 
Bergstrom, \\
KA, and TH \\
Mitchell
\end{tabular} & 1997 & $\mathrm{NA}$ & NA & \\
\hline $\begin{array}{l}\text { 116-H-1 Liquid } \\
\text { Waste Disposal } \\
\text { Trench }\end{array}$ & $100-\mathrm{H}$ & & \begin{tabular}{|l|} 
GSSI SIR \\
10 with \\
$100 \& 300$ \\
MHz \\
antenna
\end{tabular} & & & & & & & & & & & \begin{tabular}{|l|} 
Locate and accurately \\
map location of inlet \\
pipe, northern lobe of \\
trench, unknown fea- \\
tures, major concentra- \\
tions of debris, pipe- \\
lines, utilities, etc., and \\
determine optimum \\
locations for core \\
penetrometer tech- \\
nology (CPT) holes \\
and test pits. \\
\end{tabular} & $12-15^{\prime}$ & BHI-00715 & \begin{tabular}{|l|} 
Bergstrom, \\
KA; \\
Mitchell, TH
\end{tabular} & Apr-96 & 39 & Yes & \\
\hline $116-\mathrm{H}-3$ & $100-\mathrm{H}$ & $1-30-92$ & \begin{tabular}{|l|} 
GSSI SRI 8 \\
with 300 \\
MHz \\
antenna
\end{tabular} & & & & & & & & & & & $\begin{array}{l}\text { Locate utilities and } \\
\text { buried debris that may } \\
\text { affect the drilling of a } \\
\text { borehole. }\end{array}$ & & $\begin{array}{l}\text { WHC-SD-EN- } \\
\text { TI-204, Rev. O }\end{array}$ & $\begin{array}{l}\text { Bergstrom, } \\
\text { KA }\end{array}$ & 1994 & 31 & Yes. & \\
\hline $\begin{array}{l}\text { 116-H-3 Dummy } \\
\text { Decon French Drain }\end{array}$ & $100-\mathrm{H}$ & & \begin{tabular}{|l|} 
GSSI SIR 8 \\
with 300 \\
MHz \\
antenna \\
\end{tabular} & & & & & & & & & & & \begin{tabular}{|l|} 
Locate french drain \\
and subsurface utilities \\
and buried debris.
\end{tabular} & & $\begin{array}{l}\text { ERC IOM } \\
\# 051553\end{array}$ & \begin{tabular}{|l|} 
Mitchell, TH \\
and KA \\
Bergstrom
\end{tabular} & 1992 & $\mathrm{NA}$ & NA & \\
\hline $\begin{array}{l}\text { 116-H-3 French } \\
\text { Drain }\end{array}$ & $100-\mathrm{H}$ & & \begin{tabular}{|l|} 
GSSI SIR \\
$10 \mathrm{~A}$ with \\
$300 \mathrm{MHz}$ \\
antenna
\end{tabular} & & & & & & & & & & & $\begin{array}{l}\text { Verify location of } \\
\text { french drain. }\end{array}$ & $14-16^{\prime}$ & $\begin{array}{l}\text { ERC IOM } \\
\# 051553\end{array}$ & \begin{tabular}{|l|} 
Mitchell, TH \\
and KA \\
Bergstrom
\end{tabular} & 1997 & $\mathrm{NA}$ & NA & \\
\hline
\end{tabular}




\begin{tabular}{|c|c|c|c|c|c|c|c|c|c|c|c|c|c|c|c|c|c|c|c|c|c|}
\hline \multirow[b]{2}{*}{ Site/Location } & \multirow[b]{2}{*}{ Area } & \multirow[b]{2}{*}{$\begin{array}{c}\text { Date(s) } \\
\text { of Survey }\end{array}$} & \multicolumn{11}{|c|}{$\begin{array}{l}\text { Geophysical Methods Used } \\
\end{array}$} & \multirow[b]{2}{*}{ Survey Objectives } & \multirow[b]{2}{*}{\begin{tabular}{|c} 
Max. \\
Depth \\
(ft)
\end{tabular}} & \multirow[b]{2}{*}{$\begin{array}{l}\text { Reference } \\
\text { (Doc. No.) }\end{array}$} & \multirow[b]{2}{*}{ Author(s) } & \multirow[b]{2}{*}{ Date } & \multicolumn{2}{|c|}{ Bibliography } & \multirow[b]{2}{*}{ Comments } \\
\hline & & & GPR & EM & IP & $\begin{array}{c}\text { Downhole } \\
\text { Seismic }\end{array}$ & $\begin{array}{c}\text { Seismic } \\
\text { (Acoustic) }\end{array}$ & Resistivity & ERT & Gravity & Magnetics & $\begin{array}{c}\text { Metal } \\
\text { Detector }\end{array}$ & Other & & & & & & \begin{tabular}{|c} 
Ref. \\
Number
\end{tabular} & Abstract? & \\
\hline $\begin{array}{l}\text { 118-H-1 Burial } \\
\text { Ground }\end{array}$ & |100-H & $99-93$ & \begin{tabular}{|l|} 
GSSI SIR 8 \\
with 300 \\
MHz \\
antenna
\end{tabular} & $\begin{array}{l}\text { Geonics } \\
\text { EM-31D }\end{array}$ & & & & & & & & & & \begin{tabular}{l|} 
Locate and map \\
trenches, pits, and \\
buried debris within \\
burial ground. Define \\
extent of anomalous \\
region on northeast \\
corner.
\end{tabular} & |10-14' & $\begin{array}{l}\text { WHC-SD-EN- } \\
\text { TI-207 (1). } \\
\text { WHC-SD-EN- } \\
\text { DP-069 (2) }\end{array}$ & \begin{tabular}{|l|} 
1) Bergstrom \\
KA, \\
Mitchell, \\
TH, and JP \\
Kiesler \\
2) Bergstrom \\
and Mitchell \\
\end{tabular} & $\begin{array}{l}\text { 1) Mar-94 } \\
\text { 2) } 1994\end{array}$ & $\begin{array}{l}\text { 1) } 24 . \\
\text { 2) } 30\end{array}$ & $\begin{array}{l}\text { 1) No. } \\
\text { 2) No. }\end{array}$ & \\
\hline $\begin{array}{l}\text { 118-H-2 Burial } \\
\text { Ground }\end{array}$ & $100-\mathrm{H}$ & $9-93$ & \begin{tabular}{|l|} 
GSSI SIR 8 \\
with 300 \\
MHz \\
antenna
\end{tabular} & \begin{tabular}{|l|} 
Geonics \\
EM-31D
\end{tabular} & & & & & & & & & & \begin{tabular}{|l|} 
Locate two vaults, \\
estimate their size, \\
determine thickness of \\
overlying fill, and \\
locate any additional \\
debris within burial \\
ground.
\end{tabular} & $10-15^{\prime}$ & $\begin{array}{l}\text { WHC-SD-EN- } \\
\text { TI-208 (1). } \\
\text { WHC-SD-EN- } \\
\text { DP-069 (2) }\end{array}$ & \begin{tabular}{|l|} 
1) KA \\
Bergstrom, \\
TH Mitchell, \\
and GJ \\
Szwartz. \\
2) Bergstrom \\
and Mitchell \\
\end{tabular} & $\begin{array}{l}\text { 1) Mar-94 } \\
\text { 2) } 1994\end{array}$ & $\begin{array}{l}\text { 1) } 23 . \\
\text { 2) } 30\end{array}$ & $\begin{array}{l}\text { 1) No. } \\
\text { 2) No. }\end{array}$ & \\
\hline \begin{tabular}{|l|}
$118-\mathrm{H}-3$ \\
Construction Burial \\
Ground
\end{tabular} & $100-\mathrm{H}$ & $9-93$ & \begin{tabular}{|l|} 
GSSI SIR 8 \\
with 300 \\
MHz \\
antenna
\end{tabular} & $\begin{array}{l}\text { Geonics } \\
\text { EM-31D }\end{array}$ & & & & & & & & & & \begin{tabular}{l|} 
Locate individual \\
trenches, concentration \\
of debris in trenches, \\
determine thickness of \\
overlying fill, and \\
locate any additional \\
debris within burial \\
zone.
\end{tabular} & $10-15 \mathrm{ft}$ & $\begin{array}{l}\text { WHC-SD-EN- } \\
\text { TI-209 (1). } \\
\text { WHC-SD-EN- } \\
\text { DP-069 (2) }\end{array}$ & \begin{tabular}{|l|} 
1) KA \\
Bergstrom, \\
TH Mitchell, \\
and GJ \\
Szwartz. 2) \\
Bergstrom \\
and Mitchell \\
\end{tabular} & $\begin{array}{l}\text { 1) Mar-94 } \\
\text { 2) } 1994\end{array}$ & \begin{tabular}{|l|} 
1) 86. 2) \\
30
\end{tabular} & $\begin{array}{l}\text { 1) No. } \\
\text { 2) No. }\end{array}$ & \\
\hline $\begin{array}{l}\text { 128-H-1 Burial } \\
\text { Ground }\end{array}$ & $100-\mathrm{H}$ & $12-93$ & \begin{tabular}{|l|} 
GSSI SIR 8 \\
with 300 \\
MHz \\
antenna
\end{tabular} & $\begin{array}{l}\text { Geonics } \\
\text { EM-31D }\end{array}$ & & & & & & & & & & $\begin{array}{l}\text { Delineate subsurface } \\
\text { features in the } 128-\mathrm{H}-1 \\
\text { burn pit that may affect } \\
\text { the emplacement of } \\
\text { soil-gas probes. }\end{array}$ & $0-11$ & $\begin{array}{l}\text { WHC-SD-EN- } \\
\text { TI-217 }\end{array}$ & \begin{tabular}{|l|} 
Szwartz, GJ; \\
Mitchell, TH \\
and KA \\
Bergstrom \\
\end{tabular} & 11-Jul-94 & 116 & Yes & \\
\hline 130-H-1 & $100-\mathrm{H}$ & & $\mathrm{x}$ & & & & & & & & & $\mathrm{x}$ & & $\begin{array}{l}\text { Locate and map under- } \\
\text { ground fuel storage } \\
\text { tanks, pipes, and } \\
\text { cables. }\end{array}$ & $15 \mathrm{ft}$ & $\begin{array}{l}\text { PNL letter } \\
\text { report to Mark } \\
\text { Morton, WHC }\end{array}$ & \begin{tabular}{|l|} 
G.A. \\
Sandness
\end{tabular} & 10-May-90 & NA & $\mathrm{NA}$ & \\
\hline 199-H4-46 & $100-\mathrm{H}$ & $1-20-92$ & \begin{tabular}{|l|} 
GSSI SRI 8 \\
with 300 \\
MHz \\
antenna
\end{tabular} & & & & & & & & & & & $\begin{array}{l}\text { Locate utilities and } \\
\text { buried debris that may } \\
\text { affect the drilling of a } \\
\text { borehole. }\end{array}$ & & $\begin{array}{l}\text { WHC-SD-EN- } \\
\text { TI-204, Rev. O }\end{array}$ & $\begin{array}{l}\text { Bergstrom, } \\
\text { KA }\end{array}$ & 1994 & 31 & Yes & \\
\hline $199-\mathrm{H} 4-47$ & $100-\mathrm{H}$ & $\begin{array}{l}1-19-92, \\
1-16-92\end{array}$ & \begin{tabular}{|l|} 
GSSI SRI 8 \\
with 300 \\
MHz \\
antenna
\end{tabular} & & & & & & & & & & & \begin{tabular}{l|} 
Locate utilities and \\
buried debris that may \\
affect the drilling of a \\
borehole.
\end{tabular} & & $\begin{array}{l}\text { WHC-SD-EN- } \\
\text { TI-204, Rev. O }\end{array}$ & \begin{tabular}{|l|} 
Bergstrom, \\
KA
\end{tabular} & 1994 & 31 & Yes & \\
\hline $199-\mathrm{H} 4-48$ & $100-\mathrm{H}$ & $1-24-92$ & \begin{tabular}{|l|} 
GSSI SRI 8 \\
with 100 \\
and 300 \\
MHz \\
antenna
\end{tabular} & & & & & & & & & & & \begin{tabular}{l|} 
Locate utilities and \\
buried debris that may \\
affect the drilling of a \\
borehole.
\end{tabular} & & $\begin{array}{l}\text { WHC-SD-EN- } \\
\text { TI-204, Rev. O }\end{array}$ & $\begin{array}{l}\text { Bergstrom, } \\
\text { KA }\end{array}$ & 1994 & 31 & Yes & \\
\hline 199-H4-49 & $100-\mathrm{H}$ & $1-20-92$ & \begin{tabular}{|l|} 
GSSI SRI \\
with 300 \\
MHz \\
antenna \\
\end{tabular} & & & & & & & & & & & $\begin{array}{l}\text { Locate utilities and } \\
\text { buried debris that may } \\
\text { affect the drilling of a } \\
\text { borehole. }\end{array}$ & & $\begin{array}{l}\text { WHC-SD-EN- } \\
\text { TI-204, Rev. O }\end{array}$ & $\begin{array}{l}\text { Bergstrom, } \\
\text { KA }\end{array}$ & 1994 & 31 & Yes & \\
\hline 199-H5-1 & $100-\mathrm{H}$ & $1-20-92$ & \begin{tabular}{|l|} 
GSSI SRI 8 \\
with 100 \\
and 300 \\
MHz \\
antenna
\end{tabular} & & & & & & & & & & & \begin{tabular}{|l|} 
Locate utilities and \\
buried debris that may \\
affect the drilling of a \\
borehole.
\end{tabular} & & $\begin{array}{l}\text { WHC-SD-EN- } \\
\text { TI-204, Rev. O }\end{array}$ & $\begin{array}{l}\text { Bergstrom, } \\
\text { KA }\end{array}$ & 1994 & 31 & Yes & \\
\hline $\begin{array}{l}\text { Proposed Flow } \\
\text { Meter Boreholes }\end{array}$ & $100-\mathrm{H}$ & & $\mathrm{x}$ & & & & & & & & & & & site borehole & & $\begin{array}{l}\text { WHC-SD-EN- } \\
\text { TI-263 }\end{array}$ & Kiesler & Dec-94 & 59 & Yes & \\
\hline
\end{tabular}




\begin{tabular}{|c|c|c|c|c|c|c|c|c|c|c|c|c|c|c|c|c|c|c|c|c|c|}
\hline \multirow[b]{2}{*}{ Site/Location } & \multirow[b]{2}{*}{ Area } & \multirow[b]{2}{*}{$\begin{array}{c}\text { Date(s) } \\
\text { of Survey }\end{array}$} & \multicolumn{11}{|c|}{ Geophysical Methods Used } & \multirow[b]{2}{*}{ Survey Objectives } & \multirow[b]{2}{*}{\begin{tabular}{|c} 
Max. \\
Depth \\
(ft) \\
\end{tabular}} & \multirow[b]{2}{*}{$\begin{array}{l}\text { Reference } \\
\text { (Doc. No.) }\end{array}$} & \multirow[b]{2}{*}{ Author(s) } & \multirow[b]{2}{*}{ Date } & \multicolumn{2}{|c|}{ Bibliography } & \multirow[b]{2}{*}{ Comment: } \\
\hline & & & GPR & EM & IP & $\begin{array}{c}\text { Downhole } \\
\text { Seismic }\end{array}$ & $\begin{array}{c}\text { Seismic } \\
\text { (Acoustic) }\end{array}$ & Resistivity & ERT & Gravity & Magnetics & $\begin{array}{c}\text { Metal } \\
\text { Detector }\end{array}$ & Other & & & & & & $\begin{array}{c}\text { Ref. } \\
\text { Number }\end{array}$ & Abstract? & \\
\hline \begin{tabular}{|l|} 
Sodium Dichromate \\
Barrel Landfill
\end{tabular} & $100-\mathrm{H}$ & & $\mathrm{x}$ & $\mathrm{x}$ & & & & & & & & & & & & $\begin{array}{l}\text { WHC-SD-EN- } \\
\text { ES-30 (1). } \\
\text { WHC-SA-2018- } \\
\text { FP (2) }\end{array}$ & $\begin{array}{l}\text { 1) Mitchell, } \\
\text { TH and KA } \\
\text { Bergstrom. } \\
\text { 2) Berstrom, } \\
\text { Mitchell, and } \\
\text { Kunk }\end{array}$ & $\begin{array}{l}\text { 1) Oct-92 } \\
\text { 2) Jul-93 }\end{array}$ & $\begin{array}{l}\text { 1) 68.2) } \\
\text { 12 }\end{array}$ & $\begin{array}{l}\text { 1) No. } \\
\text { 2) Yes }\end{array}$ & \\
\hline $\begin{array}{l}\text { Thimble, 'south of } \\
116-\mathrm{H}-2\end{array}$ & $100-\mathrm{H}$ & $8 / 93$ & $\mathrm{x}$ & $\mathrm{x}$ & & & & & & & & & & $\begin{array}{l}\begin{array}{l}\text { Locate the buried } \\
\text { thimble. }\end{array} \\
\text { the }\end{array}$ & & $\begin{array}{l}\text { WHC-SD-EN- } \\
\text { TI-210 (1). } \\
\text { WHC-SD-EN- } \\
\text { DP-069 (2) }\end{array}$ & \begin{tabular}{|l} 
1) \\
Bergstrom, \\
KA. \\
2) Bergstrom \\
and Mitchell
\end{tabular} & $\begin{array}{l}\text { 1) } 7 / 8 / 1994 . \\
\text { 2) } 1994\end{array}$ & $\begin{array}{l}\text { 1) } 26 . \\
\text { 2) } 30\end{array}$ & $\begin{array}{l}\text { 1) Yes. } \\
\text { 2) No. }\end{array}$ & \\
\hline 116-K-2 Borehole & $100-\mathrm{K}$ & & $\mathrm{x}$ & & & & & & & & & & & Site borehole & & $\begin{array}{l}\text { WHC-SD-EN- } \\
\text { TI-092 }\end{array}$ & $\begin{array}{l}\text { Bergstrom } \\
\text { and Mitchell }\end{array}$ & Jan-93 & 3 & No. & \\
\hline $130-\mathrm{K}-1,2$ & $100-\mathrm{K}$ & & $\mathrm{x}$ & & & & & & & & & ? & & $\begin{array}{l}\text { Locate and map } \\
\text { underground fuel } \\
\text { storage tanks, pipes, } \\
\text { and cables. }\end{array}$ & $15 \mathrm{ft}$ & $\begin{array}{l}\text { PNL letter } \\
\text { report to Ron } \\
\text { Shuck, WHO }\end{array}$ & \begin{tabular}{|l|}
$\begin{array}{l}\text { G.A. } \\
\text { Sandness }\end{array}$ \\
\end{tabular} & 16-Aug-89 & NA & NA & \\
\hline 199-K-106A & $100-\mathrm{K}$ & $12-16-93$ & $\mathrm{x}$ & & & & & & & & & & & borehole siting & & $\begin{array}{l}\text { WHC-SD-EN- } \\
\text { TI-230 }\end{array}$ & Mitchell, TH & Jul-94 & 89 & Yes. & \\
\hline $\mid 199-\mathrm{K}-107 \mathrm{~A}$ & $100-\mathrm{K}$ & $12-16-93$ & $\mathrm{x}$ & & & & & & & & & & & borehole siting & & $\begin{array}{l}\text { WHC-SD-EN- } \\
\text { TI-229 }\end{array}$ & Mitchell, TH & \begin{tabular}{|l|l|} 
Nov-94 \\
\end{tabular} & 91 & Yes. & \\
\hline 199-K-108A & $100-\mathrm{K}$ & $12-16-93$ & $\mathrm{x}$ & & & & & & & & & & & borehole siting & & $\begin{array}{l}\text { WHC-SD-EN- } \\
\text { TI-228 }\end{array}$ & \begin{tabular}{|l|} 
Bergstrom \\
KA, and \\
Mitchell, TH \\
\end{tabular} & Feb-94 & 21 & Yes. & \\
\hline $\begin{array}{l}\text { 199-K-109A, 199-K- } \\
\text { 110A, and 199-K- } \\
\text { 111A boreholes }\end{array}$ & $100-\mathrm{K}$ & $3-22-94$ & \begin{tabular}{|l|} 
GSSI SRI 8 \\
with 300 \\
MHz \\
antenna \\
\end{tabular} & & & & & & & & & & & $\begin{array}{l}\text { Locate subsurface } \\
\text { obstructions that may } \\
\text { affect the drilling. }\end{array}$ & & $\begin{array}{l}\text { WHC-SD-EN- } \\
\text { TI-253 }\end{array}$ & Mitchell, TH & 1995 & 94 & Yes. & \\
\hline 199-K-32A\&B & $100-\mathrm{K}$ & $\begin{array}{l}4-29-92 \\
4-27-92\end{array}$ & \begin{tabular}{|l|} 
GSSI SRI 8 \\
with 300 \\
MHz \\
antenna
\end{tabular} & & & & & & & & & & & $\begin{array}{l}\text { Locate possible } \\
\text { underground utilities. } \\
\text { Investigate the pro- } \\
\text { posed drill site for } \\
\text { subsurface anomalies } \\
\text { that would be detri- } \\
\text { mental to the well. } \\
\end{array}$ & & $\begin{array}{l}\text { WHC-SD-EN- } \\
\text { TI-204, Rev. O }\end{array}$ & $\begin{array}{l}\text { Bergstrom, } \\
\text { KA }\end{array}$ & 1994 & 31 & Yes. & \\
\hline $199-\mathrm{K}-34$ & $100-\mathrm{K}$ & $5-7-92$ & \begin{tabular}{|l|} 
GSSI SRI 8 \\
with 300 \\
MHz \\
antenna
\end{tabular} & & & & & & & & & & & $\begin{array}{l}\text { Locate utilities and } \\
\text { buried debris that may } \\
\text { affect drilling of } \\
\text { borehole. }\end{array}$ & & $\begin{array}{l}\text { WHC-SD-EN- } \\
\text { TI-204, Rev. O }\end{array}$ & $\begin{array}{l}\text { Bergstrom, } \\
\text { KA }\end{array}$ & 1994 & 31 & Yes. & \\
\hline $199-\mathrm{K}-35$ & $100-\mathrm{K}$ & $5-7-92$ & \begin{tabular}{|l|} 
GSSI SRI 8 \\
with 300 \\
MHz \\
antenna
\end{tabular} & & & & & & & & & & & $\begin{array}{l}\text { Locate utilities and } \\
\text { buried debris that may } \\
\text { affect drilling of } \\
\text { borehole. }\end{array}$ & & $\begin{array}{l}\text { WHC-SD-EN- } \\
\text { TI-204, Rev. O }\end{array}$ & \begin{tabular}{|l} 
Bergstrom, \\
KA
\end{tabular} & 1994 & 31 & Yes. & \\
\hline $199-\mathrm{K}-36$ & $100-\mathrm{K}$ & $5-18-92$ & \begin{tabular}{|l|} 
GSSI SRI 8 \\
with 300 \\
MHz \\
antenna
\end{tabular} & & & & & & & & & & & $\begin{array}{l}\text { Locate utilities and } \\
\text { buried debris that may } \\
\text { affect drilling of } \\
\text { borehole. }\end{array}$ & & $\begin{array}{l}\text { WHC-SD-EN- } \\
\text { TI-204, Rev. O }\end{array}$ & \begin{tabular}{|l} 
Bergstrom, \\
KA
\end{tabular} & 1994 & 31 & Yes. & \\
\hline 116-KE-1 Well Site & $100-\mathrm{KE}$ & & $\begin{array}{l}\text { GSSI SIR } \\
10 \mathrm{~A} \text { with } \\
300 \mathrm{MHz} \\
\text { antenna }\end{array}$ & & & & & & & & & & & $\begin{array}{l}\text { Map utilities, pipelines, } \\
\text { and other subsurface } \\
\text { features. }\end{array}$ & & $\begin{array}{l}\text { ERC IOM } \\
\# 064927\end{array}$ & \begin{tabular}{|l} 
Mitchell, TH \\
and KA \\
Bergstrom
\end{tabular} & 1998 & NA & $\mathrm{NA}$ & \\
\hline 116-KE-3 Well Site & 100-KE & & \begin{tabular}{|l|} 
GSSI SIR \\
$10 \mathrm{~A}$ with \\
$300 \mathrm{MHz}$ \\
antenna
\end{tabular} & & & & & & & & & & & $\begin{array}{l}\text { Map utilities, pipelines, } \\
\text { and other subsurface } \\
\text { features. }\end{array}$ & & $\begin{array}{l}\text { ERC IOM } \\
\# 064927\end{array}$ & $\begin{array}{l}\text { Mitchell, TH } \\
\text { and KA } \\
\text { Bergstrom }\end{array}$ & 1998 & NA & NA & \\
\hline
\end{tabular}




\begin{tabular}{|c|c|c|c|c|c|c|c|c|c|c|c|c|c|c|c|c|c|c|c|c|c|}
\hline \multirow[b]{2}{*}{ Site/Location } & \multirow[b]{2}{*}{ Area } & \multirow[b]{2}{*}{$\left|\begin{array}{c|}\text { Date(s) } \\
\text { of Survey }\end{array}\right|$} & \multicolumn{11}{|c|}{$\begin{array}{l}\text { Geophysical Methods Used } \\
\end{array}$} & \multirow[b]{2}{*}{ Survey Objectives } & \multirow[b]{2}{*}{$\begin{array}{l}\text { Max. } \\
\text { Depth } \\
\text { (ft) }\end{array}$} & \multirow[b]{2}{*}{$\begin{array}{l}\text { Reference } \\
\text { (Doc. No.) }\end{array}$} & \multirow[b]{2}{*}{ Author(s) } & \multirow[b]{2}{*}{ Date } & \multicolumn{2}{|c|}{ Bibliography } & \multirow[b]{2}{*}{ Comments } \\
\hline & & & GPR & $\mathrm{EM}$ & IP & $\begin{array}{c}\text { Downhole } \\
\text { Seismic }\end{array}$ & $\begin{array}{c}\text { Seismic } \\
\text { (Acoustic) }\end{array}$ & Resistivity & ERT & Gravity & Magnetics & \begin{tabular}{|c|} 
Metal \\
Detector
\end{tabular} & Other & & & & & & \begin{tabular}{|c|} 
Ref. \\
Number \\
\end{tabular} & Abstract? & \\
\hline \begin{tabular}{|l}
$120-\mathrm{KE}-3$ Filter \\
Water Facility
\end{tabular} & 100-KE & & \begin{tabular}{|l|} 
GSSI SIR \\
10A with \\
300 and \\
550 MHz \\
antenna \\
\end{tabular} & & & & & & & & & & & \begin{tabular}{|l|} 
Locate and map extent \\
of trench.
\end{tabular} & & BHI-00180 & \begin{tabular}{|l|} 
Bergstrom, \\
KA: \\
Mitchell, \\
TH; Bolin, \\
DJ
\end{tabular} & Apr-95 & 32 & Yes & \\
\hline 130-KE-1A \& B & 100-KE & & $\mathrm{x}$ & & & & & & & & & $\mathrm{x}$ & & \begin{tabular}{|l} 
Locate and map \\
underground fuel \\
storage tanks, pipes, \\
and cables.
\end{tabular} & $15 \mathrm{ft}$ & \begin{tabular}{|l} 
PNL letter \\
report to Mark \\
Morton, WHO
\end{tabular} & \begin{tabular}{|l|} 
G.A. \\
Sandness
\end{tabular} & 10-May-90 & $\mathrm{NA}$ & NA & \\
\hline $116-\mathrm{KW}-3 \mathrm{~A}$ & 100-KW & \begin{tabular}{|l|}
$10-5-92$ \\
\end{tabular} & & & & & & & & & & & & borehole siting & & $\begin{array}{l}\text { WHC-SD-EN- } \\
\text { TI-095 }\end{array}$ & \begin{tabular}{|l|} 
Mitchell TH \\
and KA \\
Bergstrom
\end{tabular} & 01-Jan-93 & 74 & No. & \\
\hline $116-\mathrm{KW}-4 \mathrm{~A}$ & 100-KW & \begin{tabular}{|l|l|}
$10-6-92$ \\
\end{tabular} & & & & & & & & & & & & \begin{tabular}{|l|} 
borehole siting \\
\end{tabular} & & $\begin{array}{l}\text { WHC-SD-EN- } \\
\text { TI-093 }\end{array}$ & \begin{tabular}{|l|}
$\begin{array}{l}\text { Mitchell TH } \\
\text { and KA } \\
\text { Bergstrom }\end{array}$ \\
\end{tabular} & 01-Jan-93 & 73 & No. & \\
\hline 130-KW-1A \& B & 100-KW & & $\mathrm{x}$ & & & & & & & & & $\mathrm{x}$ & & $\begin{array}{l}\begin{array}{l}\text { Locate and map } \\
\text { underground fuel } \\
\text { storage tanks, pipes, } \\
\text { and cables. }\end{array} \\
\end{array}$ & $15 \mathrm{ft}$ & \begin{tabular}{|l|} 
PNL letter \\
report to Mark \\
Morton, WHO
\end{tabular} & \begin{tabular}{|l|} 
G.A. \\
Sandness
\end{tabular} & 10-May-90 & $\mathrm{NA}$ & $\mathrm{NA}$ & \\
\hline 100-N-G5-1 & $100-\mathrm{N}$ & \begin{tabular}{|l|l|l|l|l|} 
\\
\end{tabular} & \begin{tabular}{|l|} 
GSSI SIR 8 \\
with \\
300-MHz \\
antenna \\
\end{tabular} & & & & & & & & & & & \begin{tabular}{|l|} 
Locate excavation for \\
and underground tank \\
that hal been removed. \\
Locate well site within \\
excavation.
\end{tabular} & & $\begin{array}{l}\text { WHC-SD-EN- } \\
\text { TI-204, Rev. O }\end{array}$ & \begin{tabular}{|l} 
Bergstrom, \\
KA
\end{tabular} & 1994 & 31 & Yes. & \\
\hline $100-\mathrm{N}$-LFT & $100-\mathrm{N}$ & & $\mathrm{x}$ & & & & & & & & & $\mathrm{x}$ & & \begin{tabular}{|l|} 
Locate and map \\
underground fuel \\
storage tanks, pipes, \\
and cables.
\end{tabular} & $15 \mathrm{ft}$ & \begin{tabular}{|l|} 
PNL letter \\
report to Ron \\
Shuck, WHO
\end{tabular} & \begin{tabular}{|l} 
G.A. \\
Sandness
\end{tabular} & 27-Sep-90 & $\mathrm{NA}$ & $\mathrm{NA}$ & \\
\hline 100-N-SS-27,28 & $100-\mathrm{N}$ & & $\mathrm{x}$ & & & & & & & & & $\mathrm{x}$ & & $\begin{array}{l}\text { Locate and map } \\
\text { underground fuel } \\
\text { storage tanks, pipes, } \\
\text { and cables. }\end{array}$ & $15 \mathrm{ft}$ & $\begin{array}{l}\text { PNL letter } \\
\text { report to Ron } \\
\text { Shuck, WHO }\end{array}$ & \begin{tabular}{|l} 
G.A. \\
Sandness
\end{tabular} & 27-Sep-90 & $\mathrm{NA}$ & $\mathrm{NA}$ & \\
\hline $116-\mathrm{N}-2$ & $100-\mathrm{N}$ & 11-20-92 & $x$ & & & & & & & & & & & borehole siting & & $\begin{array}{l}\text { WHC-SD-EN- } \\
\text { TI-088 }\end{array}$ & \begin{tabular}{|l|} 
Bergstrom, \\
KA and TH \\
Mitchell
\end{tabular} & Jan-93 & 7 & No. & \\
\hline $119 \mathrm{~N}$ & $100-\mathrm{N}$ & $11-10-92$ & $\mathrm{x}$ & & & & & & & & & & & borehole siting & & $\begin{array}{l}\text { WHC-SD-EN- } \\
\text { TI-090 }\end{array}$ & \begin{tabular}{|l|} 
Bergstrom, \\
KAand TH \\
Mitchell
\end{tabular} & Jan-93 & 4 & No. & \\
\hline $1322 \mathrm{~N}$ & $100-\mathrm{N}$ & 11-19-92 & $\mathrm{x}$ & & & & & & & & & & & borehole siting & & $\begin{array}{l}\text { WHC-SD-EN- } \\
\text { TI-089 }\end{array}$ & 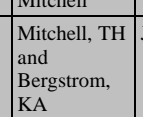 & Jan-93 & 71 & No. & \\
\hline $182-\mathrm{N}-\mathrm{DT}-1,2,3$ & $100-\mathrm{N}$ & & $\mathrm{x}$ & & & & & & & & & $\mathrm{x}$ & & \begin{tabular}{|l} 
Locate and map \\
underground fuvel \\
storage tanks, pipes, \\
and cables.
\end{tabular} & $15 \mathrm{ft}$ & \begin{tabular}{|l|} 
PNL letter \\
report to Ron \\
Shuck, WHO
\end{tabular} & \begin{tabular}{|l|} 
G.A. \\
Sandness
\end{tabular} & 27-Sep-90 & $\mathrm{NA}$ & NA & \\
\hline $\mathrm{N}-80$ & $100-\mathrm{N}$ & 6-19-92 & \begin{tabular}{|l|} 
GSSI SIR 8 \\
with \\
300-MHz \\
antenna
\end{tabular} & & & & & & & & & & & \begin{tabular}{|l} 
borehole siting \\
\end{tabular} & & $\begin{array}{l}\text { WHC-SD-EN- } \\
\text { TI-204, Rev. O }\end{array}$ & $\begin{array}{l}\text { Bergstrom, } \\
\text { KA }\end{array}$ & 1994 & 21 & Yes. & \\
\hline South Pond & $100-\mathrm{N}$ & \begin{tabular}{|l|l|}
$11-2-92$ & \\
\end{tabular} & $\mathrm{x}$ & & & & & & & & & & & borehole siting & & $\begin{array}{l}\text { WHC-SD-EN- } \\
\text { TI-094 }\end{array}$ & \begin{tabular}{|l|} 
Bergstrom, \\
KA and TH \\
Mitchell
\end{tabular} & Jan-93 & 2 & No. & \\
\hline
\end{tabular}




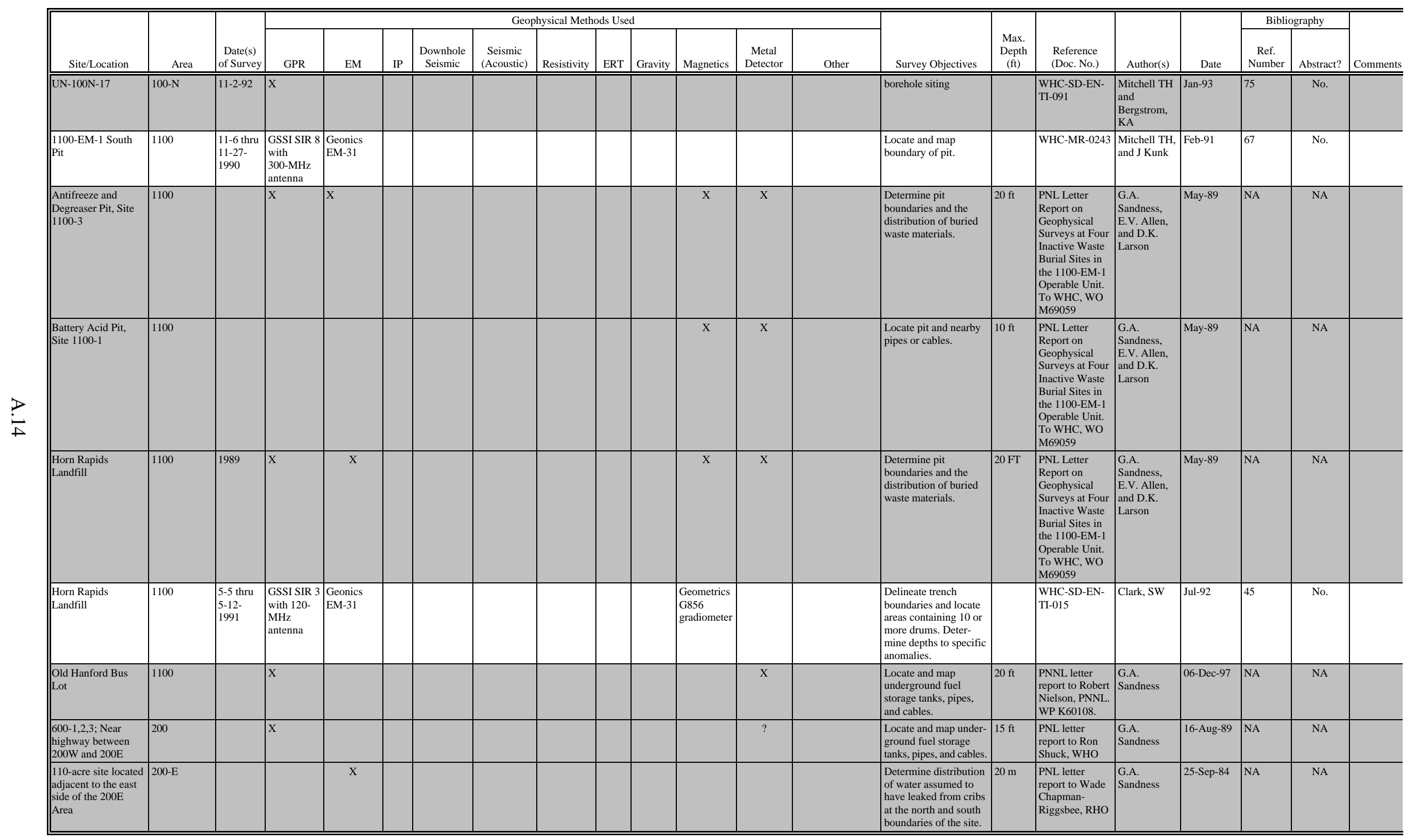




\begin{tabular}{|c|c|c|c|c|c|c|c|c|c|c|c|c|c|c|c|c|c|c|c|c|c|}
\hline \multirow{2}{*}{ Site/Location } & \multirow[b]{2}{*}{ Area } & \multirow[b]{2}{*}{\begin{tabular}{|c|} 
Date(s) \\
of Survey
\end{tabular}} & \multicolumn{11}{|c|}{$\begin{array}{l}\text { Geophysical Methods Used } \\
\end{array}$} & \multirow[b]{2}{*}{ Survey Objectives } & \multirow[b]{2}{*}{$\begin{array}{l}\text { Max. } \\
\text { Depth } \\
\text { (ft) }\end{array}$} & \multirow[b]{2}{*}{$\begin{array}{l}\text { Reference } \\
\text { (Doc. No.) }\end{array}$} & \multirow[b]{2}{*}{ Author(s) } & \multirow[b]{2}{*}{ Date } & \multicolumn{2}{|c|}{ Bibliography } & \multirow[b]{2}{*}{ Comments } \\
\hline & & & GPR & EM & IP & $\begin{array}{c}\text { Downhole } \\
\text { Seismic }\end{array}$ & $\begin{array}{c}\text { Seismic } \\
\text { (Acoustic) }\end{array}$ & Resistivity & ERT & Gravity & Magnetics & $\begin{array}{l}\text { Metal } \\
\text { Detector }\end{array}$ & Other & & & & & & \begin{tabular}{|c|} 
Ref. \\
Number
\end{tabular} & Abstract? & \\
\hline 200-E Burial Ground & $200-\mathrm{E}$ & & $\bar{x}$ & & & & & & & & & & & $\begin{array}{l}\begin{array}{l}\text { Conduct } \\
\text { reconnaissance to } \\
\text { locate trenches. }\end{array} \\
\end{array}$ & $15 \mathrm{ft}$ & $\begin{array}{l}\text { PNL Memo to } \\
\text { Bob Kasper }\end{array}$ & \begin{tabular}{|l|} 
G.A. \\
Sandness and \\
C.S. Kimball \\
\end{tabular} & 15 -Oct-82 & $\mathrm{NA}$ & $\mathrm{NA}$ & \\
\hline 216-B-2-2 Ditch & $200-\mathrm{E}$ & & \begin{tabular}{|l|} 
GSSI SIR \\
10A with \\
200-MHz \\
antenna \\
\end{tabular} & \begin{tabular}{|l|} 
Geonics \\
EM-31
\end{tabular} & & & & & & & & & & $\begin{array}{l}\text { Support excavation } \\
\text { permits and locate } \\
\text { ditch. }\end{array}$ & & \begin{tabular}{|l|} 
ERC IOM \# \\
051520
\end{tabular} & \begin{tabular}{|l|} 
Mitchell, TH \\
and KA \\
Bergstrom \\
\end{tabular} & 1997,1999 & $\mathrm{NA}$ & $\mathrm{NA}$ & \\
\hline 216-B-3-1 Ditch & $200-\mathrm{E}$ & & \begin{tabular}{|l|} 
GSSI SIR 8 \\
with 300 - \\
MHz \\
antenna
\end{tabular} & \begin{tabular}{|l|} 
Geonics \\
EM-31D
\end{tabular} & & & & & & & & & & $\begin{array}{l}\text { Verify the staked } \\
\text { location of the ditch. }\end{array}$ & $8-18 \mathrm{ft}$ & $\begin{array}{l}\text { WHC-SD-EN- } \\
\text { TI-281 }\end{array}$ & \begin{tabular}{|l} 
Bergstrom, \\
KA
\end{tabular} & 13-Sep-94 & 27 & Yes. & \\
\hline 216-B-3-3 Ditch & $200-\mathrm{E}$ & & \begin{tabular}{|l|} 
GSSI SIR \\
10A with \\
200-MHz \\
antenna
\end{tabular} & & & & & & & & & & & \begin{tabular}{|l|} 
Support excavation \\
permits for test pits and \\
map edges of ditch.
\end{tabular} & & None (1) & \begin{tabular}{|l|} 
Mitchell, TH \\
and KA \\
Bergstrom
\end{tabular} & 1999 & $\mathrm{NA}$ & $\mathrm{NA}$ & \\
\hline $216-\mathrm{C}-1$ Crib & $200-\mathrm{E}$ & & $\mathrm{x}$ & & & & & & & & & & & $\begin{array}{l}\text { Locate crib and related } \\
\text { pipes. }\end{array}$ & $15 \mathrm{ft}$ & $\begin{array}{l}\text { PNL Memo } \\
\text { from T.J. } \\
\text { McLaughlin }\end{array}$ & \begin{tabular}{|l|} 
T.J. \\
McLaughlin
\end{tabular} & 20-Jan-87 & $\mathrm{NA}$ & $\mathrm{NA}$ & \\
\hline 9 test sites & $200-\mathrm{E}$ & & $\mathrm{x}$ & & & & & & & & & & & $\begin{array}{l}\text { Test ability of GPR to } \\
\text { detect and map buried } \\
\text { pipes and cables. }\end{array}$ & $15 \mathrm{ft}$ & \begin{tabular}{|l|} 
PNL Memo to \\
Roger Keck
\end{tabular} & \begin{tabular}{|l|} 
G.A. \\
Sandness
\end{tabular} & 16-Nov-83 & $\mathrm{NA}$ & $\mathrm{NA}$ & \\
\hline \begin{tabular}{|l|} 
Air Duct at PUREX \\
Building
\end{tabular} & $200-\mathrm{E}$ & & $\mathrm{x}$ & & & & & & & & & & & \begin{tabular}{|l|} 
Detect possible voids. \\
\end{tabular} & $15 \mathrm{ft}$ & \begin{tabular}{|l|} 
PNL Memo to \\
W.E. Simpson
\end{tabular} & \begin{tabular}{|l|} 
G.A. \\
Sandness and \\
C.S. Kimball
\end{tabular} & 28-Mar-83 & $\mathrm{NA}$ & $\mathrm{NA}$ & \\
\hline B Ditch & $200-\mathrm{E}$ & & $\mathrm{x}$ & $\mathrm{x}$ & & & & & & & & & & $\begin{array}{l}\text { Detect possible shallow } \\
\text { water layer. }\end{array}$ & $20 \mathrm{ft}$ & & \begin{tabular}{|l|} 
G. Sandness \\
\end{tabular} & 1986 & $\mathrm{NA}$ & NA & \\
\hline B-Pond & $200-\mathrm{E}$ & & \begin{tabular}{|l|} 
GSSI SIR \\
10A with \\
200-MHz \\
antenna \\
\end{tabular} & \begin{tabular}{|l|} 
Geonics \\
EM-31
\end{tabular} & & & & & & & & & & \begin{tabular}{l|} 
Support excavation \\
permits for test pits and \\
one borehole and map \\
original surface of \\
pond.
\end{tabular} & & None (1) & \begin{tabular}{|l|} 
Mitchell, TH \\
and KA \\
Bergstrom
\end{tabular} & 1999 & $\mathrm{NA}$ & NA & \\
\hline Gable Mtn. Pond & $200-\mathrm{E}$ & & \begin{tabular}{|l|} 
GSSI SIR \\
10A with \\
200-MHz \\
antenna
\end{tabular} & \begin{tabular}{|l|} 
Geonics \\
EM-31
\end{tabular} & & & & & & & & & & \begin{tabular}{|l|} 
Support excavation \\
permits for test pits and \\
map original surface of \\
pond.
\end{tabular} & & None (1) & \begin{tabular}{|l|} 
Mitchell, TH \\
and KA \\
Bergstrom
\end{tabular} & 1999 & $\mathrm{NA}$ & $\mathrm{NA}$ & \\
\hline $\begin{array}{l}\text { Gable Mtn. Pond, } \\
\text { North of 200-E }\end{array}$ & $200-\mathrm{E}$ & & & & & & & \begin{tabular}{|l|} 
Soil test \\
R-60 D.C. \\
Earth Resis- \\
tivity Meter \\
and Schlum- \\
berger \\
electrode \\
configura- \\
tion
\end{tabular} & & $\begin{array}{l}\text { Worden } \\
\text { Gravity } \\
\text { Meter }\end{array}$ & \begin{tabular}{|l|} 
Geometrics \\
G-816 \\
Proton \\
Precession \\
Magneto- \\
meter
\end{tabular} & & & \begin{tabular}{|l|} 
Delineate geologic \\
controls for potential \\
groundwater move- \\
ment from Gable Mtn. \\
Pond to the \\
Rattlesnake Ridge \\
Interbed.
\end{tabular} & & $\begin{array}{l}\text { RHO-SA-239 } \\
\text { (1). RHO-ST- } \\
38 \text { (2). }\end{array}$ & \begin{tabular}{|l|} 
1) BA \\
Moore. \\
2) Strait, SR \\
and Moore, \\
BA
\end{tabular} & \begin{tabular}{|l|} 
1) Sep- \\
1982. \\
2) Dec- \\
1982
\end{tabular} & 1) 96. & $\begin{array}{l}\text { 1) Yes. } \\
\text { 2) Yes. }\end{array}$ & \\
\hline Hot Semiworks & $200-\mathrm{E}$ & & $\mathrm{x}$ & & & & & & & & & & & $\begin{array}{l}\text { Map underground } \\
\text { pipes and cables. } \\
\text { Examine cribs. }\end{array}$ & $16 \mathrm{ft}$ & \begin{tabular}{|l|} 
PNL letter \\
report to Robert \\
R. Utecht, RHO \\
\end{tabular} & \begin{tabular}{|l|} 
G.A. \\
Sandness
\end{tabular} & 16-Jun-87 & $\mathrm{NA}$ & $\mathrm{NA}$ & \\
\hline \begin{tabular}{l|} 
Proposed 120-acre \\
site for shallow land \\
grout disposal. East \\
of PUREX and AW \\
Tank Farm. \\
Designated Site C.
\end{tabular} & $200-\mathrm{E}$ & & $\mathrm{x}$ & $\mathrm{x}$ & & & & & & & $\mathrm{x}$ & & & \begin{tabular}{|l|} 
Determine whether site \\
contained buried \\
manmade structures, \\
objects, or materials.
\end{tabular} & $20 \mathrm{ft}$ & \begin{tabular}{|l|} 
PNL letter \\
report to Greg \\
Campbell, RHO
\end{tabular} & \begin{tabular}{|l|} 
G.A. \\
Sandness
\end{tabular} & 21-Feb-84 & $\mathrm{NA}$ & $\mathrm{NA}$ & \\
\hline
\end{tabular}




\begin{tabular}{|c|c|c|c|c|c|c|c|c|c|c|c|c|c|c|c|c|c|c|c|c|c|}
\hline \multirow[b]{2}{*}{$\begin{array}{l}\text { Site/Location } \\
\end{array}$} & \multirow[b]{2}{*}{ Area } & \multirow[b]{2}{*}{\begin{tabular}{|c}
$\begin{array}{c}\text { Date(s) } \\
\text { of Survey }\end{array}$ \\
\end{tabular}} & \multicolumn{11}{|c|}{ Geophysical Methods Used } & \multirow[b]{2}{*}{ Survey Objectives } & \multirow[b]{2}{*}{$\begin{array}{c}\text { Max. } \\
\text { Depth } \\
\text { (ft) }\end{array}$} & \multirow[b]{2}{*}{$\begin{array}{l}\text { Reference } \\
\text { (Doc. No.) }\end{array}$} & \multirow[b]{2}{*}{ Author(s) } & \multirow[b]{2}{*}{ Date } & \multicolumn{2}{|c|}{ Bibliography } & \multirow[b]{2}{*}{ Comments } \\
\hline & & & GPR & $\mathrm{EM}$ & IP & $\begin{array}{c}\text { Downhole } \\
\text { Seismic } \\
\end{array}$ & $\begin{array}{c}\text { Seismic } \\
\text { (Acoustic) }\end{array}$ & Resistivity & ERT & Gravity & Magnetics & \begin{tabular}{|r|} 
Metal \\
Detector \\
\end{tabular} & Other & & & & & & $\begin{array}{c}\text { Ref. } \\
\text { Number }\end{array}$ & $\begin{array}{l}\text { Abstract? } \\
\end{array}$ & \\
\hline $\begin{array}{l}\begin{array}{l}\text { Proposed 5.8-acre } \\
\text { tank farm site } \\
\text { located just west of } \\
\text { the A Tank farm }\end{array} \\
\text { the farm }\end{array}$ & $200-\mathrm{E}$ & & $\mathrm{X}$ & $\overline{\mathrm{x}}$ & & & & & & & $\overline{\bar{x}}$ & $\overline{\mathrm{x}}$ & & 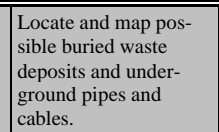 & $20 \mathrm{ft}$ & & G. Sandness & 1984 & $\overline{\mathrm{NA}}$ & $\overline{\mathrm{NA}}$ & \\
\hline $\begin{array}{l}\text { Proposed 70-acre } \\
\text { site for shallow land } \\
\text { grout disposal. } \\
\text { Directly north of Hot } \\
\text { Semi Works, north } \\
\text { of 7th St. } \\
\text { Desigated Site A. }\end{array}$ & $200-\mathrm{E}$ & & $\mathrm{x}$ & $\mathrm{x}$ & & & & & & & $\mathrm{x}$ & & & $\begin{array}{l}\text { Determine whether site } \\
\text { contained buried } \\
\text { manmade structures, } \\
\text { objects, or materials. }\end{array}$ & $20 \mathrm{ft}$ & \begin{tabular}{|l|} 
PNL letter \\
report to Greg \\
Campbell, RHO
\end{tabular} & \begin{tabular}{|l|} 
G.A.A. \\
Sandness
\end{tabular} & 21-Feb-84 & $\mathrm{NA}$ & $\mathrm{NA}$ & \\
\hline $\begin{array}{l}\begin{array}{l}\text { Proposed con- } \\
\text { struction sites A \& B } \\
\text { near B Plant. }\end{array} \\
\text { nat }\end{array}$ & $200-\mathrm{E}$ & & $\mathrm{x}$ & $\mathrm{x}$ & & & & & & & & $\mathrm{x}$ & & \begin{tabular}{|l|}
$\begin{array}{l}\text { Detect and map any } \\
\text { buried waste deposits, } \\
\text { pipelines, or cables. }\end{array}$ \\
\end{tabular} & $20 \mathrm{ft}$ & \begin{tabular}{|l} 
PNL letter \\
report to Claude \\
Denson, KEH \\
\end{tabular} & \begin{tabular}{|l} 
G.A. \\
Sandness
\end{tabular} & 12-Jul-83 & $\mathrm{NA}$ & $\mathrm{NA}$ & \\
\hline \begin{tabular}{|l|} 
Proposed site for \\
HWVP. $25 \mathrm{mi}$ WSW \\
of B Plant
\end{tabular} & $200-\mathrm{E}$ & & $\mathrm{x}$ & $\mathrm{x}$ & & & & & & & $\mathrm{x}$ & $\mathrm{x}$ & & \begin{tabular}{|l|}
$\begin{array}{l}\text { Detect and map any } \\
\text { buried waste deposits, } \\
\text { pipelines, or cables. }\end{array}$ \\
\end{tabular} & $15 \mathrm{ft}$ & $\begin{array}{l}\begin{array}{l}\text { PNL letter } \\
\text { report to Jack } \\
\text { Cloud, KEH }\end{array} \\
\end{array}$ & \begin{tabular}{|l|} 
G.A. \\
Sandness
\end{tabular} & |02-Apr-86 & $\mathrm{NA}$ & $\mathrm{NA}$ & \\
\hline \begin{tabular}{|l|} 
Tank Mockup Test \\
Site Near Hot Semi \\
Works
\end{tabular} & $200-\mathrm{E}$ & & & & & & & & $\mathrm{x}$ & & & & & $\begin{array}{l}\text { 1) Test the capabilities } \\
\text { and limitations of ERT } \\
\text { to determine its utility } \\
\text { to detect and map pleaks } \\
\text { from underground } \\
\text { storage tanks during } \\
\text { waste removal proces- } \\
\text { ses, and for long-term } \\
\text { vadose zone monitor- } \\
\text { ing. 2) Develop and } \\
\text { demonstrate the ability } \\
\text { to place vertical elec- } \\
\text { trode arrays (VEA) } \\
\text { with he cone penentro- } \\
\text { meter technology } \\
\text { (CPT) to depths below } \\
\text { existing single-shell } \\
\text { tanks. }\end{array}$ & & $\begin{array}{l}\text { WHC-SD-EN- } \\
\text { THP-57 (1). } \\
\text { WHC-SA-3035- } \\
\text { FP/CONF- } \\
\text { Fo6477-3 (2). }\end{array}$ & \begin{tabular}{|l|}
1 \\
Narbutovskih, \\
Sr. \\
2). \\
Narbutovskih, \\
SM.. et. al.
\end{tabular} & $\begin{array}{l}\text { 1) Apr-96. } \\
\text { 2) Jan-96. }\end{array}$ & $\begin{array}{l}\text { 1) } 97 . \\
\text { 2) } 101 .\end{array}$ & $\begin{array}{l}\text { 1) Yes. } \\
\text { 2) Yes. }\end{array}$ & \\
\hline $\begin{array}{l}\text { TK-SQ-151, 152; } \\
\text { B-Plant }\end{array}$ & $200-\mathrm{E}$ & & $\mathrm{x}$ & & & & & & & & & ? & & $\begin{array}{l}\text { Locate and map } \\
\text { underground fuel } \\
\text { storage tanks, pipes, } \\
\text { and cables. }\end{array}$ & $15 \mathrm{ft}$ & $\begin{array}{l}\text { PND letter } \\
\text { report to Ron } \\
\text { Shuck, WHO }\end{array}$ & $\begin{array}{l}\text { G.A. } \\
\text { Sandness }\end{array}$ & 16-Aug-89 & NA & NA & \\
\hline $\begin{array}{l}\text { Vicinity of Tank } \\
\text { 101-A in the 241-A } \\
\text { Tank Farm }\end{array}$ & $200-\mathrm{E}$ & & $\mathrm{x}$ & & & & & & & & & & & \begin{tabular}{|l|} 
Investigate \\
effectivenss of GPR \\
to detect and map \\
buried pipelines in the \\
tank farm. \\
\end{tabular} & $15 \mathrm{ft}$ & $\begin{array}{l}\text { PNL Memos to } \\
\text { Bill Jordan }\end{array}$ & \begin{tabular}{|l} 
1) G. \\
Sandness and \\
C.S. Kimball \\
2) G. \\
Sandness \\
\end{tabular} & \begin{tabular}{|l} 
1) 16-Dec- \\
81 2 $18-$ \\
Mar-82
\end{tabular} & $\begin{array}{l}\text { 1) } \mathrm{NA} \\
\text { 2) } \mathrm{NA}\end{array}$ & $\begin{array}{l}\text { 1) } \mathrm{NA} \\
\text { 2) } \mathrm{NA}\end{array}$ & \\
\hline 216-U-1/2 pipeline & $200-\mathrm{W}$ & & $\mathrm{x}$ & & & & & & & & & & & $\begin{array}{l}\text { Locate and determine } \\
\text { the depth of the } 216-\mathrm{U}- \\
1 / 2 \text { pipeline. } \\
\text {. }\end{array}$ & & $\begin{array}{l}\text { WHC-SD-EN- } \\
\text { TI-262 }\end{array}$ & $\begin{array}{l}\text { Fassett, J; } \\
\text { Berstrom, } \\
\text { KA }\end{array}$ & 22-Aug-96 & 52 & Yes. & \\
\hline \begin{tabular}{|l|}
$216-\mathrm{U}-4$ and $216-$ \\
$\mathrm{U}-16$
\end{tabular} & $200-\mathrm{W}$ & & $\mathrm{x}$ & & & & & & & & & & & $?$ & & $\begin{array}{l}\text { WHC-SD-EN- } \\
\text { TI-176 }\end{array}$ & \begin{tabular}{|l|}
$\begin{array}{l}\text { Bergstrom } \\
\text { and Mitchell }\end{array}$ \\
\end{tabular} & 01-Sep-93 & 16 & No. & \\
\hline \begin{tabular}{|l|}
$216-\mathrm{U}-8$ and $-\mathrm{U}-12$ \\
transfer line
\end{tabular} & $200-\mathrm{W}$ & & $\mathrm{x}$ & $\mathrm{x}$ & & & & & & & & & & \begin{tabular}{|l} 
Locate the vitrified \\
clay piep, locate bends \\
in the pipo, and locate \\
possible entilities or \\
other pipelines.
\end{tabular} & & $\begin{array}{l}\text { WHC-SD-EN- } \\
\text { TI-241 }\end{array}$ & $\begin{array}{l}\text { Bergstrom, } \\
\text { KA }\end{array}$ & 05-Dec-94 & 28 & Yes. & \\
\hline
\end{tabular}




\begin{tabular}{|c|c|c|c|c|c|c|c|c|c|c|c|c|c|c|c|c|c|c|c|c|c|}
\hline \multirow[b]{2}{*}{$\begin{array}{l}\text { Site/Location } \\
\end{array}$} & \multirow[b]{2}{*}{ Area } & \multirow[b]{2}{*}{\begin{tabular}{|c|}
$\begin{array}{c}\text { Date(s) } \\
\text { of Survey }\end{array}$ \\
\end{tabular}} & \multicolumn{11}{|c|}{$\begin{array}{l}\text { Geophysical Methods Used } \\
\end{array}$} & \multirow[b]{2}{*}{ Survey Objectives } & \multirow[b]{2}{*}{\begin{tabular}{|c|} 
Max. \\
Depth \\
(ft) \\
\end{tabular}} & \multirow[b]{2}{*}{$\begin{array}{l}\begin{array}{l}\text { Reference } \\
\text { (Doc. No.). }\end{array} \\
\end{array}$} & \multirow[b]{2}{*}{ Author(s) } & \multirow[b]{2}{*}{ Date } & \multicolumn{2}{|c|}{ Bibliography } & \multirow[b]{2}{*}{ Comments } \\
\hline & & & GPR & EM & IP & $\begin{array}{c}\text { Downhole } \\
\text { Seismic }\end{array}$ & $\begin{array}{c}\text { Seismic } \\
\text { (Acoustic) }\end{array}$ & Resistivity & ERT & Gravity & Magnetics & $\begin{array}{c}\text { Metal } \\
\text { Detector }\end{array}$ & Other & & & & & & \begin{tabular}{|c|}
$\begin{array}{c}\text { Ref. } \\
\text { Number }\end{array}$ \\
\end{tabular} & Abstract? & \\
\hline "216-Z-12 Crib & $200-\mathrm{W}$ & & $\overline{\bar{x}}$ & & & & & & & & & & & \begin{tabular}{|l|} 
ISV site. Detect \\
underground objects or \\
voids.
\end{tabular} & $\overline{20 \mathrm{ft}}$ & \begin{tabular}{|l|} 
PNL Memo to \\
Jim Buelt, PNL
\end{tabular} & $\begin{array}{l}\text { G.A.A } \\
\text { Sandness }\end{array}$ & 13-Apr-87 & $\overline{\mathrm{NA}}$ & $\overline{\mathrm{NA}}$ & \\
\hline 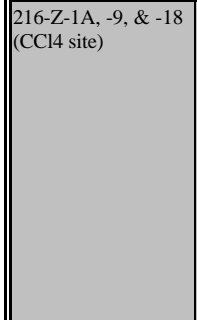 & $200-\mathrm{W}$ & & & & & $\begin{array}{l}\text { Vertical } \\
\text { Seismic } \\
\text { Profiling } \\
\text { and Rever- } \\
\text { sed VSP, } \\
\text { using jet } \\
\text { perforator } \\
\text { as source } \\
\text { and DAS-1 } \\
\text { seimograp } \\
\text { h and 3 } \\
\text { conponent } \\
\text { geophones }\end{array}$ & & & & & & & & \begin{tabular}{|l|} 
Test techniques for \\
obtaining velocity \\
control and subsurface \\
imaging capabilities.
\end{tabular} & & $\begin{array}{l}\text { WHC-SA- } \\
\text { 2304/CONF- } \\
940353-4\end{array}$ & \begin{tabular}{|l|} 
Narbutovskih, \\
SM: \\
Michelsen, $\mathrm{F}$
\end{tabular} & Feb-94 & 98 & $\begin{array}{l}\text { Yes } \\
\text { Y }\end{array}$ & \\
\hline \begin{tabular}{|l|}
$\begin{array}{l}216-Z-Z 1 A,-9, ~ \&-18 \\
\text { (CCl4 site) }\end{array}$ \\
\end{tabular} & $200-\mathrm{W}$ & & $\mathrm{x}$ & \begin{tabular}{|l|} 
Frequency \\
Domain \\
(FDEM), \\
Time \\
Domain \\
(TDEM)
\end{tabular} & $\mathrm{x}$ & & \begin{tabular}{|l|} 
Refraction, \\
Reflection
\end{tabular} & $\begin{array}{l}\text { Direct } \\
\text { Current } \\
\text { (DC) }\end{array}$ & & & & & & \begin{tabular}{|l|}
$\begin{array}{l}\text { Support demonstration } \\
\text { of a data fusion } \\
\text { workstation. }\end{array}$ \\
\end{tabular} & & \begin{tabular}{|l|} 
WHC-SD-EN- \\
TI-202 (1). \\
WHC-DD-EN- \\
AP-116 (2). \\
DOEMC/2910 \\
6-3531 (3). \\
PNL-10393 (4) \\
\end{tabular} & 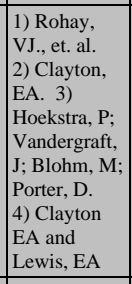 & $\begin{array}{l}\text { 1) } 9 / 28 / 199 . \\
\text { 2) } 1993 . \\
\text { 3) Au- }-93 . \\
\text { 4) Sep-95. }\end{array}$ & $\begin{array}{l}\text { 1) } 109 . \\
\text { 2) } 46 . \\
\text { 3) } 55 . \\
\text { 4) } 47 .\end{array}$ & $\begin{array}{l}\text { 1) YES. } \\
\text { 2) No. } \\
\text { 3 YES. } \\
\text { 4) YES. }\end{array}$ & \\
\hline $\begin{array}{l}\text { 216-Z-9 } 9 \text { Trench / } \\
\text { CCl4 site }\end{array}$ & $200-\mathrm{W}$ & Jan-99 & & & & & $\begin{array}{l}\text { 2-D High } \\
\text { Resolution } \\
\text { Refletion } \\
\text { using new } \\
\text { amplitude } \\
\text { versus offset } \\
\text { AVO) } \\
\text { technology }\end{array}$ & & & & & & & \begin{tabular}{|l|} 
Determinin location and \\
distribution of \\
DNAPL.
\end{tabular} & & $\begin{array}{l}\text { Informal Report } \\
\text { on Non- } \\
\text { Invasive } \\
\text { Determination } \\
\text { of the Location } \\
\text { and Distribution } \\
\text { of Free-Phase } \\
\text { Dense } \\
\text { Nonaqueous } \\
\text { Phase eivuids } \\
\text { by Siesmic } \\
\text { Reflection } \\
\text { Techniques } \\
\end{array}$ & \begin{tabular}{|l|} 
M. G. \\
Waddell, \\
W. J., \\
DDomoracki, \\
TJ Temples, \\
and J Eyer \\
\end{tabular} & Nov. 1999 & $\mathrm{NA}$ & $\mathrm{NA}$ & \\
\hline 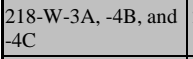 & $200-\mathrm{W}$ & & & & & & & & & & & & & & & $\begin{array}{l}\text { WHC-SD-EN- } \\
\text { TI-295 } \\
\end{array}$ & Kiesler & Aug-96 & 61 & No. & \\
\hline \begin{tabular}{|l|l}
$218-\mathrm{W}-4 \mathrm{C}$ Trench \\
$\# 4$
\end{tabular} & $200-\mathrm{W}$ & & $\mathrm{x}$ & $\mathrm{x}$ & & & & & & & & & & 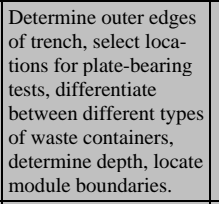 & & $\begin{array}{l}\text { WHC-SD-EN- } \\
\text { TI-285 }\end{array}$ & Kiesler & 1994 & 60 & 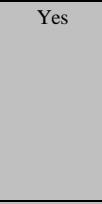 & \\
\hline 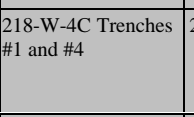 & $200-\mathrm{W}$ & & \begin{tabular}{|l|} 
with 300 \\
MHz \\
antenna
\end{tabular} & & & & & & & & & & & \begin{tabular}{|l|} 
Determine effective- \\
ness of GPR for \\
defining the depth and \\
location of barrels.
\end{tabular} & & $\begin{array}{l}\text { WHC-SD-EN- } \\
\text { TI-174 }\end{array}$ & \begin{tabular}{|l|} 
Kiesler, \\
Bergstrom \\
and Mitchell \\
\end{tabular} & 1993 & 58 & $\begin{array}{l}\text { Yes } \\
\text { Pan }\end{array}$ & \\
\hline 221- U Plant Cut & $200-\mathrm{W}$ & & $\mathrm{x}$ & & & & & & & & & & & & & $\begin{array}{l}\text { WHC-SD-EN- } \\
\text { TI-180 }\end{array}$ & \begin{tabular}{|l|}
$\begin{array}{l}\text { Kiesler and } \\
\text { Mitchell }\end{array}$ \\
\end{tabular} & Aug-93 & 57 & No. & \\
\hline
\end{tabular}




\begin{tabular}{|c|c|c|c|c|c|c|c|c|c|c|c|c|c|c|c|c|c|c|c|c|c|}
\hline \multirow[b]{2}{*}{ Site/Location } & \multirow[b]{2}{*}{ Area } & \multirow[b]{2}{*}{\begin{tabular}{|c|} 
Date(s) \\
of Survey
\end{tabular}} & \multicolumn{11}{|c|}{ Geophysical Methods Used } & \multirow[b]{2}{*}{ Survey Objectives } & \multirow{2}{*}{\begin{tabular}{|l} 
Max. \\
Depth \\
(ft)
\end{tabular}} & \multirow[b]{2}{*}{$\begin{array}{l}\text { Reference } \\
\text { (Doc. No.) }\end{array}$} & \multirow[b]{2}{*}{ Author(s) } & \multirow[b]{2}{*}{ Date } & \multicolumn{2}{|c|}{ Bibliography } & \multirow[b]{2}{*}{ Comments } \\
\hline & & & GPR & EM & IP & $\begin{array}{c}\text { Downhole } \\
\text { Seismic }\end{array}$ & $\begin{array}{c}\text { Seismic } \\
\text { (Acoustic) }\end{array}$ & Resistivity & ERT & Gravity & Magnetics & \begin{tabular}{|c|} 
Metal \\
Detector
\end{tabular} & Other & & & & & & $\begin{array}{c}\text { Ref. } \\
\text { Number }\end{array}$ & Abstract? & \\
\hline \begin{tabular}{|l|} 
221-T Building SQ \\
Tanks
\end{tabular} & $200-W$ & $4 / 4 / 94$ & \begin{tabular}{|l|} 
GSSI SIR 8 \\
with 300- \\
and 500- \\
MHz \\
antenna \\
\end{tabular} & & & & & & & & & & & $\begin{array}{l}\text { Locate subsurface } \\
\text { obstructions that may } \\
\text { affect the removal of } \\
\text { tanks and supporting } \\
\text { structures. } \\
\end{array}$ & & $\begin{array}{l}\text { WHC-SD-EN- } \\
\text { TI-223 }\end{array}$ & Mitchell, TH & 1995 & 95 & $\overline{\text { Yes. }}$ & \\
\hline $\begin{array}{l}\text { 221-U Plant (south } \\
\text { side of building) }\end{array}$ & $200-W$ & & \begin{tabular}{|l|} 
GSSI SIR \\
10A with \\
300-MHz \\
antenna
\end{tabular} & & & & & & & & & & & \begin{tabular}{|l|} 
Locate and map \\
subsurface tanks, \\
utilities, pipelines, and \\
unknown buried \\
features.
\end{tabular} & $0-14^{\prime}$ & BHI-01182 & \begin{tabular}{|l|} 
Bergstrom, \\
KA; \\
Mitchell, \\
TH; Sharpe, \\
JJ; Singleton, \\
KM
\end{tabular} & 31-May-98 & 43 & Yes & \\
\hline $\begin{array}{l}221-\mathrm{U} \text { southwest } \\
\text { corner and MO-722 }\end{array}$ & $200-W$ & & \begin{tabular}{|l|} 
GSSI SIR \\
10A with \\
300-MHz \\
antenna
\end{tabular} & & & & & & & & & & & \begin{tabular}{|l|} 
Locate subsurface \\
utilities, pipelines, and \\
other buried features.
\end{tabular} & $0-14^{\prime}$ & None (1) & \begin{tabular}{|l|} 
Mitchell TH, \\
and KA \\
Bergstrom
\end{tabular} & 1998 & $\mathrm{NA}$ & $\mathrm{NA}$ & \\
\hline \begin{tabular}{|l|} 
299-W11-232 \\
Borehole
\end{tabular} & $200-W$ & & $x$ & & & & & & & & & & & site boreholes & & $\begin{array}{l}\text { WHC-SD-EN- } \\
\text { TI-141 }\end{array}$ & \begin{tabular}{|l|} 
Mitchell and \\
Bergstrom
\end{tabular} & \begin{tabular}{|l|l|} 
Jan-94 \\
\end{tabular} & 84 & No. & \\
\hline \begin{tabular}{|l|} 
299-W15-218 \\
Borehole
\end{tabular} & $200-W$ & & $\mathrm{x}$ & & & & & & & & & & & site boreholes & & $\begin{array}{l}\text { WHC-SD-EN- } \\
\text { TI-096 }\end{array}$ & \begin{tabular}{|l|} 
Bergstrom \\
and Mitchell
\end{tabular} & Jan-93 & 6 & No. & \\
\hline \begin{tabular}{|l|} 
299-W15-219, W15- \\
220, W18-252 \\
Boreholes
\end{tabular} & $200-W$ & & $\mathrm{x}$ & & & & & & & & & & & site boreholes & & $\begin{array}{l}\text { WHC-SD-EN- } \\
\text { TI-153 }\end{array}$ & \begin{tabular}{|l|}
$\begin{array}{l}\text { Bergstrom } \\
\text { and Mitchell }\end{array}$ \\
\end{tabular} & Jun-93 & 11 & No. & \\
\hline \begin{tabular}{|l|} 
299-W15-25 and \\
W15-223 Boreholes
\end{tabular} & $200-W$ & & $\mathrm{x}$ & & & & & & & & & & & site boreholes & & $\begin{array}{l}\text { WHC-SD-EN- } \\
\text { TI-196 }\end{array}$ & \begin{tabular}{|l|} 
Mitchell and \\
Bergstrom
\end{tabular} & Nov-93 & 80 & No. & \\
\hline \begin{tabular}{|l} 
299-W15-25, 26 and \\
27 Boreholes
\end{tabular} & $200-\mathrm{W}$ & \begin{tabular}{|l|}
$6-14-93$ \\
$5-5-94$
\end{tabular} & \begin{tabular}{|l|} 
GSSI SIR 8 \\
with \\
300-MHz \\
antenna
\end{tabular} & & & & & & & & & & & $\begin{array}{l}\text { Locate subsurface } \\
\text { obstructions that may } \\
\text { affect the drilling. }\end{array}$ & & $\begin{array}{l}\text { WHC-SD-EN- } \\
\text { TI-282 }\end{array}$ & \begin{tabular}{|l|} 
Mitchell, TH \\
\end{tabular} & Jan-95 & 92 & Yes. & \\
\hline \begin{tabular}{|l|} 
299-W18-252 cone \\
penetrometer site, \\
CPT-4; adjacent to \\
and west of borehole
\end{tabular} & $200-\mathrm{W}$ & $5-12-93$ & \begin{tabular}{|l|} 
GSSI SIR 8 \\
with \\
300-MHz \\
antenna
\end{tabular} & & & & & & & & & & & $\begin{array}{l}\text { Locate subsurface } \\
\text { obstructions that may } \\
\text { affect cone penetro- } \\
\text { meter work. }\end{array}$ & & $\begin{array}{l}\text { WHC-SD-EN- } \\
\text { TI-162 }\end{array}$ & Mitchell, TH & 1993 & 82 & Yes & \\
\hline \begin{tabular}{|l|l} 
Candidate ISV test \\
site adjacent to west \\
side of 241-T Tank \\
Farm??
\end{tabular} & $200-\mathrm{W}$ & & $\mathrm{x}$ & & & & & & & & & & & $\begin{array}{l}\begin{array}{l}\text { Locate assumed } \\
\text { backfilled trench. }\end{array} \\
\end{array}$ & $14 \mathrm{ft}$ & \begin{tabular}{|l|} 
PNL Memo to \\
Craig \\
Timmerman, \\
PNL
\end{tabular} & \begin{tabular}{|l|} 
G.A. \\
Sandness
\end{tabular} & $27-\mathrm{Feb}-85$ & $\mathrm{NA}$ & $\mathrm{NA}$ & \\
\hline \begin{tabular}{|l|}
$\begin{array}{l}\text { CPT-BT-23 } \\
\text { borehole }\end{array}$ \\
\end{tabular} & $200-W$ & & $\mathrm{x}$ & & & & & & & & & & & site boreholes & & $\begin{array}{l}\text { WHC-SD-EN- } \\
\text { TI-193 }\end{array}$ & \begin{tabular}{|l|} 
Mitchell and \\
Bergstrom
\end{tabular} & Mar-94 & 76 & No & \\
\hline REDOX BIdg & $200-W$ & & $\mathrm{x}$ & & & & & & & & & & & $\begin{array}{l}\text { Look for possible void } \\
\text { near the front steps of } \\
\text { the building. }\end{array}$ & $15 \mathrm{ft}$ & PNL Memo & G. Sandness & 1992 & $\mathrm{NA}$ & $\mathrm{NA}$ & \\
\hline \begin{tabular}{|l|}
300 Area Burial \\
Grounds (300 North)
\end{tabular} & 300 & & \begin{tabular}{|l|} 
Prototype \\
system \\
with \\
$1000 \mathrm{MHz}$ \\
antenna, \\
and a \\
commercial \\
system
\end{tabular} & & & & \begin{tabular}{|l|} 
3-D \\
Holographic \\
imaging (test \\
site only)
\end{tabular} & & & & \begin{tabular}{|l|} 
Proton \\
Precision \\
and cesium \\
vapor \\
magnetome \\
ters
\end{tabular} & \begin{tabular}{|l|}
2 \\
commercia \\
1 systems
\end{tabular} & & $\begin{array}{l}\text { Locate, map, and } \\
\text { define buried waste. } \\
\text { Locate and define the } \\
\text { gross composition of } \\
\text { waste materials. }\end{array}$ & & PNL-2557 & \begin{tabular}{|l|} 
Phillips, SJ; \\
Aims, LL; \\
Fitzner, RE; \\
Gee, GW; \\
Sandness, \\
GA: and \\
Simmons, \\
CS
\end{tabular} & 1980 & 106 & Yes & \\
\hline
\end{tabular}




\begin{tabular}{|c|c|c|c|c|c|c|c|c|c|c|c|c|c|c|c|c|c|c|c|c|c|}
\hline \multirow[b]{2}{*}{ Site/Location } & \multirow[b]{2}{*}{ Area } & \multirow[b]{2}{*}{\begin{tabular}{|c}
$\begin{array}{c}\text { Date(s) } \\
\text { of Survey }\end{array}$ \\
\end{tabular}} & \multicolumn{11}{|c|}{ Geophysical Methods Used } & \multirow[b]{2}{*}{ Survey Objectives } & \multirow[b]{2}{*}{$\begin{array}{c}\text { Max. } \\
\text { Depth } \\
\text { (ft) }\end{array}$} & \multirow[b]{2}{*}{$\begin{array}{l}\text { Reference } \\
\text { (Doc. No.) }\end{array}$} & \multirow[b]{2}{*}{ Author(s) } & \multirow[b]{2}{*}{ Date } & \multicolumn{2}{|c|}{ Bibliography } & \multirow[b]{2}{*}{ Comments } \\
\hline & & & GPR & EM & IP & $\begin{array}{c}\text { Downhole } \\
\text { Seismic }\end{array}$ & $\begin{array}{c}\text { Seismic } \\
\text { (Acoustic) }\end{array}$ & Resistivity & ERT & Gravity & Magnetics & \begin{tabular}{|c|} 
Metal \\
Detector
\end{tabular} & Other & & & & & & \begin{tabular}{|c|} 
Ref. \\
Number
\end{tabular} & Abstract? & \\
\hline \begin{tabular}{|l}
30 Area Process \\
Sewers and \\
radioactive liquid \\
waste sewers
\end{tabular} & 300 & & $\begin{array}{l}\begin{array}{l}\text { GSSI SIR } \\
\text { System } 7 \\
\text { with } \\
\text { 300-MHz } \\
\text { antenna }\end{array} \\
\end{array}$ & $\begin{array}{l}\begin{array}{l}\text { Geonics } \\
\text { EM-31 }\end{array} \\
\end{array}$ & & & & & & & & & & \begin{tabular}{|l|} 
Confirm the locations \\
of the sewers as shown \\
on existing maps or to \\
otherwise accurately \\
determine their loca- \\
tions, and second, to \\
attempt to identify \\
locations of possible \\
leaks.
\end{tabular} & & \begin{tabular}{l|l} 
EMO-1032 \\
\end{tabular} & $\begin{array}{l}\begin{array}{l}\text { Sandness, } \\
\text { GA }\end{array} \\
\end{array}$ & |Mar-91 & 1110 & $\begin{array}{l}\text { Yes } \\
\end{array}$ & \\
\hline $300-F-5$ & 300 & 1991 & & & & & Reflection & & & & & & & \begin{tabular}{l|} 
Two main targets: the \\
water table at roughly \\
$40 \mathrm{ft}$, and the Ringold \\
Lower mud at about \\
$180 \mathrm{ft}$
\end{tabular} & & $\begin{array}{l}\text { WHC-SD-EN- } \\
\text { TI-069 (1) } \\
\text { WHC-SD-EN- } \\
\text { DP-059 (2). } \\
\text { WHC-SD-EN- } \\
\text { TI-120 (3) }\end{array}$ & 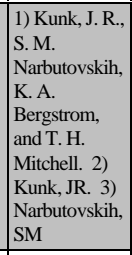 & $\begin{array}{l}\text { 1) Jan-93. } \\
\text { 2) } 1993 . \\
\text { 3) Jul-1993 }\end{array}$ & $\begin{array}{l}\text { 1) } 63 . \\
\text { 2) } 64 . \\
\text { 3) } 97\end{array}$ & $\begin{array}{l}\text { 1) No. } \\
\text { 2) no. } \\
\text { 3) yes. }\end{array}$ & \\
\hline 307 Process Trench & 300 & & $\begin{array}{l}\text { GSSI SIR } \\
\text { System 7 } \\
\text { with 100-- } \\
\text { 120-, and } \\
\text { 300-MHz } \\
\text { antenna }\end{array}$ & $\begin{array}{l}\text { Geonics } \\
\text { EM-31 }\end{array}$ & & & & & & & & & & \begin{tabular}{|l|} 
Detect underground \\
pipes, cables, or orther \\
subsurface features, \\
and to locate the \\
boundaries of the \\
trench.
\end{tabular} & & $\begin{array}{l}\text { WHC-SD-EN- } \\
\text { TI-062 }\end{array}$ & $\begin{array}{l}\text { Sandness, } \\
\text { GA }\end{array}$ & Nov-91 & 112 & No & \\
\hline 307 Retention Basin & 300 & & \begin{tabular}{|l} 
GSSI SIR \\
System 7 \\
with 120- \\
and $00-$ \\
MHz \\
antenna \\
anten
\end{tabular} & & & & & & & & & & & \begin{tabular}{|l|}
$\begin{array}{l}\text { Detect underground } \\
\text { pipes, cables, or other } \\
\text { subsurface features. }\end{array}$ \\
\end{tabular} & & $\begin{array}{l}\text { WHC-SD-EN- } \\
\text { TI-062 }\end{array}$ & $\begin{array}{l}\text { Sandness, } \\
\text { GA }\end{array}$ & Nov-91 & 112 & No & \\
\hline $311-1,2$ & 300 & & $\mathrm{x}$ & & & & & & & & & $\mathrm{x}$ & & $\begin{array}{l}\text { Locate and map } \\
\text { underground fuul } \\
\text { storage tanks, pipes, } \\
\text { and cables. }\end{array}$ & $15 \mathrm{ft}$ & \begin{tabular}{|l} 
PNL letter \\
report to Ron \\
Shuck, WHO
\end{tabular} & $\begin{array}{l}\begin{array}{l}\text { G.A.A. } \\
\text { Sandness }\end{array} \\
\end{array}$ & 16-Aug-89 & $\mathrm{NA}$ & $\mathrm{NA}$ & \\
\hline $\begin{array}{l}316-4 \text { (300 North } \\
\text { Crib) }\end{array}$ & 300 & & \begin{tabular}{|l} 
GSSI SIR \\
10 ith \\
300 MHz \\
antenna
\end{tabular} & \begin{tabular}{|l|} 
Geonics \\
EM-31D
\end{tabular} & & & & & & & & & & $\begin{array}{l}\text { Map tanks' location } \\
\text { and determine depth of } \\
\text { burial, map associated } \\
\text { plumbing for tanks and } \\
\text { nearby well 699-S6- } \\
\text { EA. }\end{array}$ & $10-18^{\prime}$ & BHI-00212 & \begin{tabular}{|l|} 
Bergstrom \\
KA; Mitchell \\
TH; Bolin BJ
\end{tabular} & May-95 & 34 & No & \\
\hline $325-1$ & 300 & & $\mathrm{x}$ & & & & & & & & & $\mathrm{x}$ & & $\begin{array}{l}\text { Locate and map } \\
\text { underground fuel } \\
\text { storage ataks, pipes, } \\
\text { and cables. }\end{array}$ & $15 \mathrm{ft}$ & \begin{tabular}{|l} 
PNL letter \\
report to Mark \\
Morton, WHO
\end{tabular} & \begin{tabular}{|l|}
$\begin{array}{l}\text { G.A.A. } \\
\text { Sandness }\end{array}$ \\
\end{tabular} & 10-May-90 & $\mathrm{NA}$ & $\mathrm{NA}$ & \\
\hline \begin{tabular}{|l}
384 Powerhouse \\
Fuel Oil Day Yanks
\end{tabular} & 300 & & \begin{tabular}{|l} 
GSSI SIR \\
10A with \\
300 and \\
400-MHz \\
antenna
\end{tabular} & & & & & & & & & & & $\begin{array}{l}\begin{array}{l}\text { Map utilities, pipelines, } \\
\text { and other subsurface } \\
\text { features. }\end{array} \\
\end{array}$ & & None (1) & \begin{tabular}{|l|}
$\begin{array}{l}\text { Mitchell, TH } \\
\text { and KA } \\
\text { Bergstrom }\end{array}$ \\
Bergte
\end{tabular} & 1998,1999 & $\mathrm{NA}$ & $\mathrm{NA}$ & \\
\hline \begin{tabular}{|l} 
384 Powerhouse \\
USTs
\end{tabular} & 300 & & \begin{tabular}{|l} 
GSSI SIR \\
10A with \\
300-MHz \\
antenna
\end{tabular} & & & & & & & & & & & $\begin{array}{l}\begin{array}{l}\text { Map utilities, pipelines, } \\
\text { and other subsurface } \\
\text { features. }\end{array} \\
\end{array}$ & $0-12^{\prime}$ & None (1) & \begin{tabular}{|l|} 
Mitchell, TH \\
and KA \\
Bergstrom
\end{tabular} & 1998, 1999 & $\mathrm{NA}$ & $\mathrm{NA}$ & \\
\hline
\end{tabular}




\begin{tabular}{|c|c|c|c|c|c|c|c|c|c|c|c|c|c|c|c|c|c|c|c|c|c|}
\hline \multirow[b]{2}{*}{ Site/Location } & \multirow[b]{2}{*}{ Area } & \multirow[b]{2}{*}{$\begin{array}{c}\text { Date(s) } \\
\text { of Survey }\end{array}$} & \multicolumn{11}{|c|}{$\begin{array}{l}\text { Geophysical Methods Used } \\
\end{array}$} & \multirow[b]{2}{*}{ Survey Objectives } & \multirow[b]{2}{*}{$\begin{array}{l}\text { Max. } \\
\text { Depth } \\
\text { (ft) }\end{array}$} & \multirow[b]{2}{*}{$\begin{array}{l}\text { Reference } \\
\text { (Doc. No.) }\end{array}$} & \multirow[b]{2}{*}{ Author(s) } & \multirow[b]{2}{*}{ Date } & \multicolumn{2}{|c|}{ Bibliography } & \multirow[b]{2}{*}{ Comments } \\
\hline & & & GPR & EM & IP & $\begin{array}{c}\text { Downhole } \\
\text { Seismic }\end{array}$ & $\begin{array}{c}\text { Seismic } \\
\text { (Acoustic) }\end{array}$ & Resistivity & ERT & Gravity & Magnetics & $\begin{array}{c}\text { Metal } \\
\text { Detector }\end{array}$ & Other & & & & & & \begin{tabular}{c|} 
Ref. \\
Number
\end{tabular} & Abstract? & \\
\hline \begin{tabular}{|l|}
$618-1$ Burial Ground \\
\end{tabular} & 300 & & $\begin{array}{l}\text { GSSI SIR } \\
10 A \text { with } \\
300-M H z \\
\text { antenna }\end{array}$ & & & & & & & & & & & \begin{tabular}{|l|} 
Map subsurface pits, \\
trenches, buried debris, \\
etc. within landfill.
\end{tabular} & & $\begin{array}{l}\text { BHI-00297, } \\
\text { Rev. 1 }\end{array}$ & \begin{tabular}{l|l}
$\begin{array}{l}\text { Mitchell, TH } \\
\text { and KA } \\
\text { Bergstrom }\end{array}$ & 1 \\
\end{tabular} & 1995 & $\overline{\mathrm{NA}}$ & $\overline{\mathrm{NA}}$ & \\
\hline \begin{tabular}{|l|}
$618-10$ and $618-11$ \\
(300 Area North and \\
Wye Burial \\
Grounds)
\end{tabular} & 300 & & $\mathrm{X}$ & & & & \begin{tabular}{|l|} 
Reflection \& \\
Refraction
\end{tabular} & & & & $\mathrm{x}$ & $\mathrm{x}$ & & $\begin{array}{l}\text { Conduct geophysical } \\
\text { exploration. }\end{array}$ & & \begin{tabular}{|l|} 
BNWL-SA- \\
$5494 /$ CONF- \\
$750967-15$
\end{tabular} & \begin{tabular}{|l|} 
Phillips, SJ; \\
Raymond, \\
JR
\end{tabular} & 1975 & 103 & Yes & \\
\hline \begin{tabular}{|l|}
$618-10$ and $618-11$ \\
(300 Area North and \\
Wye Burial \\
Grounds)
\end{tabular} & 300 & & \begin{tabular}{|l|} 
Calspan \\
Corp. with \\
1000-MHz \\
antenna \\
\end{tabular} & & & & \begin{tabular}{|l|} 
Reflection \& \\
Refraction
\end{tabular} & & & & $\mathrm{x}$ & $\mathrm{x}$ & $\begin{array}{l}\text { Mercury- } \\
\text { cadmium-telluride } \\
\text { infrared detector }\end{array}$ & $\begin{array}{l}\text { Detect the compo- } \\
\text { sition, size, distribu- } \\
\text { tion, and depth of } \\
\text { buried objects and } \\
\text { characterizing the } \\
\text { sediments in which } \\
\text { they are buried. }\end{array}$ & & \begin{tabular}{|l|} 
BNWL-SA- \\
54941 / CONF- \\
$750967-16$
\end{tabular} & \begin{tabular}{|l|l} 
Phillips, SJ; & 1 \\
Raymond, \\
JR
\end{tabular} & 1975 & 104 & Yes & \\
\hline \begin{tabular}{|l|}
$618-10$ and $618-11$ \\
(300 Area North and \\
Wye Burial \\
Grounds)
\end{tabular} & 300 & & $\mathrm{x}$ & & & & $\mathrm{x}$ & & & & $\mathrm{x}$ & $\mathrm{X}$ & & 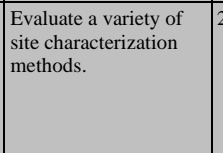 & $20 \mathrm{ft}$ & BNWL-2184 & \begin{tabular}{|l|} 
Phillips, SJ; \\
Reisenauer, \\
AE; Rickard, \\
WH, and \\
Sandness, \\
GA
\end{tabular} & $\begin{array}{r}1977 \\
\end{array}$ & 105 & No. & \\
\hline $\begin{array}{l}\text { 618-10 Burial } \\
\text { Ground }\end{array}$ & 300 & & \begin{tabular}{|l|} 
GSSI SRI \\
System 8 \\
(4800) with \\
$300-\mathrm{MHz}$ \\
antenna \\
\end{tabular} & \begin{tabular}{|l} 
Geonics \\
EM-31D
\end{tabular} & & & & & & & & & & \begin{tabular}{|l|} 
Delineate boundaries \\
of individual trenches, \\
locate highest concen- \\
trations of debris, \\
determine thickness of \\
filll, locate vertical pipe \\
units.
\end{tabular} & $10-18^{\prime}$ & $\begin{array}{l}\text { BHI-00291- } \\
\text { REV.1 }\end{array}$ & \begin{tabular}{|l|} 
Bergstrom, \\
KA; Bolin, \\
DJ; Mitchell, \\
TH
\end{tabular} & Sep-97 & 42 & 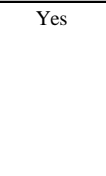 & \\
\hline $\begin{array}{l}\text { 618-11 Burial } \\
\text { Ground }\end{array}$ & 300 & & \begin{tabular}{|l|} 
GSSI SRI \\
System 10 \\
with $300-$ \\
MHz \\
antenna
\end{tabular} & \begin{tabular}{|l|} 
Geonics \\
EM-31D
\end{tabular} & & & & & & & & & & \begin{tabular}{|l|} 
Delineate boundaries \\
of individual trenches, \\
locate highest concen- \\
trations of debris, \\
determine thickness of \\
fill, locate vertical pipe \\
units and caissons. \\
\end{tabular} & $10-18^{\prime}$ & $\begin{array}{l}\text { BHI-00291- } \\
\text { REV.1 }\end{array}$ & \begin{tabular}{|l|} 
Bergstrom, \\
KA; Bolin, \\
DJ; Mitchell, \\
TH
\end{tabular} & Sep-97 & 42 & Yes & \\
\hline 618-13 Mound & 300 & & \begin{tabular}{|l} 
GSSI SIR \\
10 with \\
$300 \mathrm{MHz}$ \\
antenna
\end{tabular} & $\begin{array}{l}\text { Geonics } \\
\text { EM-31D }\end{array}$ & & & & & & & & & & \begin{tabular}{l|}
$\begin{array}{l}\text { Locate possible drums } \\
\text { or other detectable } \\
\text { waste. }\end{array}$ \\
\end{tabular} & $10-18^{\prime}$ & BHI-00212 & \begin{tabular}{|l|l|} 
Bergstrom & I \\
KA; Mitchell \\
TH; Bolin BJ
\end{tabular} & May-95 & 34 & No & \\
\hline \begin{tabular}{|c|}
$618-2$ Burial Ground \\
\end{tabular} & 300 & & & & & & & & & & & & & & & $\begin{array}{l}\text { BHI-00297, } \\
\text { Rev. 1 }\end{array}$ & \begin{tabular}{|l|}
$\begin{array}{l}\text { Mitchell, TH } \\
\text { and KA } \\
\text { Bergstrom }\end{array}$ \\
\end{tabular} & 1995 & $\mathrm{NA}$ & $\mathrm{NA}$ & \\
\hline \begin{tabular}{|c|}
$618-3$ Burial Ground \\
\end{tabular} & 300 & & \begin{tabular}{|l|} 
GSSI SIR \\
10A with \\
300 MHz \\
antenna
\end{tabular} & \begin{tabular}{|l|} 
Geonics \\
EM-31D
\end{tabular} & & & & & & & & & & \begin{tabular}{|l|} 
Map subsurface pits, \\
trenches, buried debris, \\
etc. within landfill.
\end{tabular} & & $\begin{array}{l}\text { BHI-00297, } \\
\text { Rev. 1 }\end{array}$ & \begin{tabular}{|l|l}
$\begin{array}{l}\text { Mitchell, TH } \\
\text { and KA } \\
\text { Bergstrom }\end{array}$ & 1 \\
\end{tabular} & 1995 & $\mathrm{NA}$ & NA & \\
\hline 618-4 Burial Ground & 300 & & \begin{tabular}{|l|} 
GSSI SIR \\
System 7 \\
with 300- \\
MHz \\
antenna
\end{tabular} & $\begin{array}{l}\text { Geonics } \\
\text { EM-32 }\end{array}$ & & & & & & & & & & \begin{tabular}{l|} 
Identify burial trenches \\
and pits, determine the \\
depth of fill, and locate \\
waste materials, \\
including any that \\
might be outside the \\
perimeter fences.
\end{tabular} & & EMO-1032 & \begin{tabular}{|l|} 
Sandness, \\
GA
\end{tabular} & Mar-91 & 110 & 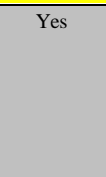 & \\
\hline
\end{tabular}




\begin{tabular}{|c|c|c|c|c|c|c|c|c|c|c|c|c|c|c|c|c|c|c|c|c|c|}
\hline \multirow[b]{2}{*}{ Site/Location } & \multirow[b]{2}{*}{ Area } & \multirow[b]{2}{*}{$\begin{array}{c}\text { Date(s) } \\
\text { of Survey }\end{array}$} & \multicolumn{11}{|c|}{ Geophysical Methods Used } & \multirow[b]{2}{*}{ Survey Objectives } & \multirow[b]{2}{*}{\begin{tabular}{|c|} 
Max. \\
Depth \\
(ft)
\end{tabular}} & \multirow[b]{2}{*}{$\begin{array}{l}\text { Reference } \\
\text { (Doc. No.) }\end{array}$} & \multirow[b]{2}{*}{ Author(s) } & \multirow[b]{2}{*}{ Date } & \multicolumn{2}{|c|}{ Bibliography } & \multirow[b]{2}{*}{ Comments } \\
\hline & & & GPR & EM & IP & $\begin{array}{c}\text { Downhole } \\
\text { Seismic }\end{array}$ & $\begin{array}{c}\text { Seismic } \\
\text { (Acoustic) }\end{array}$ & Resistivity & ERT & Gravity & Magnetics & $\begin{array}{l}\text { Metal } \\
\text { Detector }\end{array}$ & Other & & & & & & \begin{tabular}{c|} 
Ref. \\
Number
\end{tabular} & Abstract? & \\
\hline \begin{tabular}{|l|}
$618-4$ Burial Ground \\
\end{tabular} & 300 & & $\begin{array}{l}\text { GSSI SIR } \\
\text { System 7 } \\
\text { with } \\
120-\text { MHz } \\
\text { antenna }\end{array}$ & & & & & & & & \begin{tabular}{|l} 
Geometrics \\
G-856 \\
Proton \\
Precession \\
Magneto- \\
meter
\end{tabular} & \begin{tabular}{|l} 
Fisher \\
Tw6
\end{tabular} & & \begin{tabular}{|l|} 
Determine depths of \\
fill, identify subsurface \\
structures such as \\
trenches and pits, and \\
detect and map buried \\
waste materials and \\
other significant \\
features.
\end{tabular} & & $\begin{array}{l}\text { WHC-SD-EN- } \\
\text { TI-061 }\end{array}$ & $\begin{array}{l}\text { Sandness, } \\
\text { GA }\end{array}$ & Sep-91 & 1111 & No & \\
\hline \begin{tabular}{|c|}
$618-4$ Burial Ground \\
\end{tabular} & 300 & & & \begin{tabular}{|l|} 
Electrical \\
Offset \\
Logging \\
(EOL); \\
surface to \\
borehole \\
electro- \\
magnetic \\
induction
\end{tabular} & & & & & & & & & & $\begin{array}{l}\text { Delineate depth and } \\
\text { lateral extent of } \\
\text { conductive zones. }\end{array}$ & & \begin{tabular}{|l} 
PNNL Letter \\
Report (1). \\
Client Report \\
for Battelle \\
Pacific \\
Northwest \\
"618-4 Burial \\
Ground" (2).
\end{tabular} & \begin{tabular}{|l|} 
2) Murray, \\
Last, Chien, \\
and Sandness \\
1) WMI \\
International, \\
Inc. \\
\end{tabular} & \begin{tabular}{|l|l|} 
1) $9 / 16 / 99$ \\
2) $8 / 1 / 1999$
\end{tabular} & \begin{tabular}{|l|} 
1) $\mathrm{NA}$ \\
2) $\mathrm{NA}$
\end{tabular} & $\begin{array}{l}\text { 1) } \mathrm{NA} \\
\text { 2) } \mathrm{NA}\end{array}$ & \\
\hline \begin{tabular}{|c|}
$618-4$ Burial Ground \\
\end{tabular} & 300 & & \begin{tabular}{|l|} 
LEMA, \\
GSSI SIR \\
$10 \mathrm{~A}$ with \\
$300-\mathrm{MHz}$ \\
antenna \\
\end{tabular} & & & & & & & & & & \begin{tabular}{|l|} 
Also tested \\
RAMAC GPR \\
with 200-, 400- \\
MHz, and 1 GHz \\
antenna, and the \\
GSSI GPR unit \\
with 500-MHz \\
antenna at Little \\
Egypt.
\end{tabular} & $\begin{array}{l}\text { Support development } \\
\text { and testing of the Low- } \\
\text { Frequency Electro- } \\
\text { magnetic Array } \\
\text { (LEMA) for imaging } 3 \\
\text { sites with in the burial } \\
\text { ground. }\end{array}$ & & $\begin{array}{l}\text { PNL-SA-25908 } \\
\text { (1). Draft } \\
\text { Technology } \\
\text { Evaluation } \\
\text { Report (RL } \\
\text { 37SS41) (2) } \\
\text { None }^{(1)}{ }^{(3)}\end{array}$ & \begin{tabular}{|l|} 
1) Collins, \\
Gribble, \\
Hall, and \\
Lechelt. 2) \\
Collins, DH. \\
3) Mitchell, \\
TH and KA \\
Bergstrom. \\
\end{tabular} & $\begin{array}{l}\text { 1) Apr- } \\
\text { 1995. } \\
\text { 2) Sep- } \\
\text { 1997. } \\
\text { 3) NA }\end{array}$ & \begin{tabular}{|l|} 
1) 48. \\
2) NA \\
NA
\end{tabular} & $\begin{array}{l}\text { 1) Yes. } \\
\text { 2) No } \\
\text { 3) NA }\end{array}$ & \\
\hline \begin{tabular}{|c|}
$618-5$ Burial Ground \\
\end{tabular} & 300 & & \begin{tabular}{|l|} 
GSSI SIR \\
System 7 \\
with \\
$120-$ MHz \\
antenna \\
\end{tabular} & & & & & & & & \begin{tabular}{|l|} 
Geometrics \\
G-856 \\
Proton \\
Precession \\
Magneto- \\
meter
\end{tabular} & \begin{tabular}{|l|} 
Fisher \\
Tw6
\end{tabular} & & \begin{tabular}{|l|} 
Determine depths of \\
fill, identify subsurface \\
structures such as \\
trenches and pits, and \\
detect and map buried \\
waste materials and \\
other significant \\
features.
\end{tabular} & & $\begin{array}{l}\text { WHC-SD-EN- } \\
\text { TI-061 }\end{array}$ & \begin{tabular}{|l|} 
Sandness, \\
GA
\end{tabular} & Sep-91 & 111 & No & \\
\hline $\begin{array}{l}\text { 618-5 Burial Ground } \\
\text { Perimeter }\end{array}$ & 300 & & \begin{tabular}{|l|} 
GSSI SIR \\
System 7 \\
with \\
300-MHz \\
antenna \\
\end{tabular} & \begin{tabular}{|l} 
Geonics \\
EM-31
\end{tabular} & & & & & & & & & & $\begin{array}{l}\text { Identify burial trenches } \\
\text { and pits, determine the } \\
\text { depth of fill, and locate } \\
\text { waste materials, } \\
\text { including any that } \\
\text { might be outside the } \\
\text { perimeter fences. }\end{array}$ & & EMO-1032 & $\begin{array}{l}\text { Sandness, } \\
\text { GA }\end{array}$ & Mar-91 & 110 & Yes & \\
\hline 618-7 Burial Ground & 300 & & \begin{tabular}{|l|} 
GSSI SIR \\
10 with \\
$300-M H z$ \\
antenna
\end{tabular} & \begin{tabular}{|l|} 
Geonics \\
EM-31D
\end{tabular} & & & & & & & & & & $\begin{array}{l}\text { Delineate the bound- } \\
\text { aries of individual } \\
\text { trenches, locate the } \\
\text { highest concentrations } \\
\text { of debris within the } \\
\text { trenches, determine the } \\
\text { thickness of the fill } \\
\text { overlying the waste, } \\
\text { and evaluate and } \\
\text { resolve conflicting } \\
\text { historical documenta- } \\
\text { tion. }\end{array}$ & $10-18^{\prime}$ & BHI-00313 & \begin{tabular}{|l|} 
Bergstrom, \\
KA, TH \\
Mitchell, and \\
BJ Bolin \\
\end{tabular} & May-95 & 33 & No & \\
\hline
\end{tabular}




\begin{tabular}{|c|c|c|c|c|c|c|c|c|c|c|c|c|c|c|c|c|c|c|c|c|c|}
\hline \multirow[b]{2}{*}{ Site/Location } & \multirow[b]{2}{*}{ Area } & \multirow[b]{2}{*}{$\begin{array}{c}\text { Date(s) } \\
\text { of Survey }\end{array}$} & \multicolumn{11}{|c|}{ "Geophysical Methods Used } & \multirow[b]{2}{*}{ Survey Objectives } & \multirow{2}{*}{\begin{tabular}{|c} 
Max. \\
Depth \\
(ft)
\end{tabular}} & \multirow[b]{2}{*}{$\begin{array}{l}\text { Reference } \\
\text { (Doc. No.) }\end{array}$} & \multirow[b]{2}{*}{ Author(s) } & \multirow[b]{2}{*}{ Date } & \multicolumn{2}{|c|}{ "Bibliography } & \multirow[b]{2}{*}{ Comments } \\
\hline & & & GPR & EM & IP & \begin{tabular}{|c|} 
Downhole \\
Seismic
\end{tabular} & $\begin{array}{c}\text { Seismic } \\
\text { (Acoustic) }\end{array}$ & Resistivity & ERT & Gravity & Magnetics & $\begin{array}{l}\text { Metal } \\
\text { Detector }\end{array}$ & Other & & & & & & $\begin{array}{c}\text { Ref. } \\
\text { Number }\end{array}$ & Abstract? & \\
\hline 618-8 Burial Ground & 300 & & \begin{tabular}{|l|} 
GSSI SIR 7 \\
with 50- \\
200-MHz \\
antenna
\end{tabular} & $\mathrm{x}$ & & & & & & & $\begin{array}{l}\text { Varian } \\
\text { VIW- } \\
\text { 2302A1 } \\
\text { Cesium } \\
\text { Vapor } \\
\text { magneto- } \\
\text { meter } \\
\end{array}$ & \begin{tabular}{|l} 
Fisher \\
Tw5
\end{tabular} & & \begin{tabular}{|l|} 
Locate buried waste \\
material and determine \\
boundaries of waste \\
trenches or pits.
\end{tabular} & $15 \mathrm{ft}$ & \begin{tabular}{|l} 
PNL Letter \\
Report \\
$\# \# 002745$, to \\
Mary Sinclair
\end{tabular} & $\begin{array}{l}\text { Sandness, } \\
\text { GA }\end{array}$ & 26-Jun-87 & $\mathrm{NA}$ & $\mathrm{NA}$ & \\
\hline 618-8 Burial Ground & 300 & & \begin{tabular}{|l|} 
GSSI SIR \\
10 with \\
300-MHz \\
antenna
\end{tabular} & \begin{tabular}{|l|} 
Geonics \\
EM-31D
\end{tabular} & & & & & & & & & & $\begin{array}{l}\text { Map possible burial } \\
\text { ground, locate other } \\
\text { trenches, pits, etc., and } \\
\text { locate concentrations } \\
\text { of buried debris and } \\
\text { thickness of fill } \\
\text { overlying waste. }\end{array}$ & $10-18^{\prime}$ & BHI-00212 & \begin{tabular}{|l|} 
Bergstrom \\
KA; Mitchell \\
TH; Bolin BJ
\end{tabular} & May-95 & 34 & No & \\
\hline 618-9 Burial Ground & 300 & & \begin{tabular}{|l|} 
GSSI SIR 8 \\
with \\
300-MHz \\
antenna
\end{tabular} & & & & & & & & & & & $\begin{array}{l}\text { Determine orientation } \\
\text { of trench and map } \\
\text { boundaries. Map } \\
\text { anomalies in trench. }\end{array}$ & & WHC-MR-0271 & \begin{tabular}{|l|} 
JR Kunk and \\
Th Mitchell
\end{tabular} & Jul-91 & 62 & No & \\
\hline \begin{tabular}{|l} 
618-9, Area outside \\
fenced burial ground \\
approximately $.5 \mathrm{mi}$ \\
$\mathrm{NW}$ of the $300 \mathrm{Area}$
\end{tabular} & 300 & & $\mathrm{x}$ & & & & & & & & & & & $\begin{array}{l}\text { Check whether burial } \\
\text { trench extended } \\
\text { outside fence. }\end{array}$ & $15 \mathrm{ft}$ & $\begin{array}{l}\text { PNL letter } \\
\text { report to Cliff } \\
\text { Meinhardt, } \\
\text { RHO }\end{array}$ & \begin{tabular}{|l|} 
G.A. \\
Sandness
\end{tabular} & 16-Oct-84 & $\mathrm{NA}$ & $\mathrm{NA}$ & \\
\hline $\begin{array}{l}\text { Burial Trench West } \\
\text { of Process Trenches }\end{array}$ & 300 & & \begin{tabular}{|l|} 
GSSI SIR \\
10 with \\
300-MHz \\
antenna
\end{tabular} & \begin{tabular}{|l|} 
Geonics \\
EM-31D
\end{tabular} & & & & & & & & & & $\begin{array}{l}\text { Determine if solid } \\
\text { waste was buried in the } \\
\text { berm and map concen- } \\
\text { trations of waste, if } \\
\text { present. }\end{array}$ & $10-18^{\prime}$ & BHI-00212 & $\begin{array}{l}\text { Bergstrom } \\
\text { KA; Mitchell } \\
\text { TH; Bolin BJ }\end{array}$ & May-95 & 34 & No & \\
\hline \begin{tabular}{|l} 
Buried lithium \\
aluminate rods; \\
approx. $1000 \mathrm{ft}$ north \\
of 300 Area, east of \\
Process Trench \\
\end{tabular} & 300 & & $\mathrm{x}$ & & & & & & & & & $\mathrm{X}$ & & $\begin{array}{l}\text { Locate lithium } \\
\text { aluminate rods buried } \\
\text { in undocumented } \\
\text { location. }\end{array}$ & $16 \mathrm{ft}$ & $\begin{array}{l}\text { PNL letter } \\
\text { report to Doug } \\
\text { Lenkersdorfer, } \\
\text { WHC }\end{array}$ & \begin{tabular}{|l|} 
G.A. \\
Sandness
\end{tabular} & 10-Aug-88 & NA & NA & \\
\hline $\begin{array}{l}\text { ISV Test Site NW of } \\
300 \text { Area }\end{array}$ & 300 & & $\mathrm{x}$ & $\mathrm{x}$ & & & & & & & $\mathrm{x}$ & $\mathrm{x}$ & & $\begin{array}{l}\text { Detect any manmade } \\
\text { objects or materials. }\end{array}$ & $20 \mathrm{ft}$ & \begin{tabular}{|l} 
PNL Memo to \\
Craig \\
Timmerman, \\
PNL
\end{tabular} & \begin{tabular}{|l|} 
G.A. \\
Sandness
\end{tabular} & 03-Jun-83 & $\mathrm{NA}$ & $\mathrm{NA}$ & \\
\hline \begin{tabular}{|l} 
ISV Test Site NW of \\
300 Area
\end{tabular} & 300 & & $\mathrm{x}$ & $\mathrm{x}$ & & & & & & & $\mathrm{x}$ & $\mathrm{x}$ & & $\begin{array}{l}\text { Detect any manmade } \\
\text { objects or materials. }\end{array}$ & $20 \mathrm{ft}$ & $\begin{array}{l}\text { PNL Memo to } \\
\text { Steve Liikala }\end{array}$ & \begin{tabular}{|l|} 
G.A. \\
Sandness
\end{tabular} & 13-Aug-84 & $\mathrm{NA}$ & NA & \\
\hline Landfill 1A & 300 & & \begin{tabular}{|l|} 
GSSI SIR \\
System 7 \\
with 120- \\
and \\
300-MHz \\
antenna
\end{tabular} & \begin{tabular}{|l|} 
Geonics \\
EM-31
\end{tabular} & & & & & & & \begin{tabular}{|l|} 
Geometrics \\
G-856 \\
Proton \\
Precession \\
Magneto- \\
meter
\end{tabular} & \begin{tabular}{|l} 
Fisher \\
Tw6
\end{tabular} & & \begin{tabular}{|l|} 
Determine depths of \\
fill, identify subsurface \\
structures such as \\
trenches and pits, and \\
detect and map buried \\
waste materials and \\
other significant \\
features.
\end{tabular} & & $\begin{array}{l}\text { WHC-SD-EN- } \\
\text { TI-060 }\end{array}$ & \begin{tabular}{|l} 
Sandness, \\
GA
\end{tabular} & Apr-92 & 113 & No. & \\
\hline Landfill 1B & 300 & & & & & & & & & & & & & & & $\begin{array}{l}\text { ERC IMO } \\
\# 053474\end{array}$ & \begin{tabular}{|l|} 
Bergstrom, \\
KA, and TH \\
Mitchell \\
\end{tabular} & 1998 & $\mathrm{NA}$ & $\mathrm{NA}$ & \\
\hline
\end{tabular}




\begin{tabular}{|c|c|c|c|c|c|c|c|c|c|c|c|c|c|c|c|c|c|c|c|c|c|}
\hline \multirow[b]{2}{*}{ Site/Location } & \multirow[b]{2}{*}{ Area } & \multirow[b]{2}{*}{$\begin{array}{c}\text { Date(s) } \\
\text { of Survey }\end{array}$} & \multicolumn{11}{|c|}{ Geophysical Methods Used } & \multirow[b]{2}{*}{ Survey Objectives } & \multirow[b]{2}{*}{\begin{tabular}{|c|} 
Max. \\
Depth \\
(ft)
\end{tabular}} & \multirow[b]{2}{*}{$\begin{array}{l}\text { Reference } \\
\text { (Doc. No.) }\end{array}$} & \multirow[b]{2}{*}{ Author(s) } & \multirow[b]{2}{*}{ Date } & \multicolumn{2}{|c|}{ Bibliography } & \multirow[b]{2}{*}{ Comments } \\
\hline & & & GPR & EM & IP & $\begin{array}{c}\text { Downhole } \\
\text { Seismic }\end{array}$ & $\begin{array}{c}\text { Seismic } \\
\text { (Acoustic) }\end{array}$ & Resistivity & ERT & Gravity & Magnetics & $\begin{array}{c}\text { Metal } \\
\text { Detector }\end{array}$ & Other & & & & & & \begin{tabular}{|c|} 
Ref. \\
Number
\end{tabular} & Abstract? & \\
\hline Landfill 1B & 300 & & $\begin{array}{l}\text { GSSI SIR } \\
\text { System 7 } \\
\text { with 120- } \\
\text { and } \\
\text { 300-MHz } \\
\text { antenna } \\
\end{array}$ & $\begin{array}{l}\text { Geonics } \\
\text { EM-31 }\end{array}$ & & & & & & & \begin{tabular}{|l|} 
Geometrics \\
G-856 \\
Proton \\
Precession \\
Magneto- \\
meter \\
\end{tabular} & \begin{tabular}{|l} 
Fisher \\
Tw6
\end{tabular} & & \begin{tabular}{l|} 
Determine depths of \\
fill, identify subsurface \\
structures such as \\
trenches and pits, and \\
detect and map buried \\
waste materials and \\
other significant \\
features.
\end{tabular} & & $\begin{array}{l}\text { WHC-SD-EN- } \\
\text { TI-060 }\end{array}$ & $\begin{array}{l}\text { Sandness, } \\
\text { GA }\end{array}$ & Apr-92 & 113 & No. & \\
\hline Landfill 1C & 300 & & \begin{tabular}{|l|} 
GSSI SIR \\
System 7 \\
with 120- \\
and \\
300-MHz \\
antenna
\end{tabular} & $\begin{array}{l}\text { Geonics } \\
\text { EM-31 }\end{array}$ & & & & & & & \begin{tabular}{|l|} 
Geometrics \\
G-856 \\
Proton \\
Precession \\
Magneto- \\
meter
\end{tabular} & \begin{tabular}{|l} 
Fisher \\
Tw6
\end{tabular} & & \begin{tabular}{|l|} 
Determine depths of \\
fill, identify subsurface \\
structures such as \\
trenches and pits, and \\
detect and map buried \\
waste materials and \\
other significant \\
features. \\
\end{tabular} & & $\begin{array}{l}\text { WHC-SD-EN- } \\
\text { TI-060 }\end{array}$ & $\begin{array}{l}\text { Sandness, } \\
\text { GA }\end{array}$ & Apr-92 & 113 & No. & \\
\hline Landfilll 1D Burn Pit & 300 & & \begin{tabular}{|l|} 
GSSI SIR \\
System 7 \\
with 120- \\
and \\
300-MHz \\
antenna \\
\end{tabular} & \begin{tabular}{|l|} 
Geonics \\
EM-31
\end{tabular} & & & & & & & \begin{tabular}{|l|} 
Geometrics \\
G-856 \\
Proton \\
Precession \\
Magneto- \\
meter
\end{tabular} & \begin{tabular}{|l} 
Fisher \\
Tw6
\end{tabular} & & \begin{tabular}{|l|} 
Determine depths of \\
fill, identify subsurface \\
structures such as \\
trenches and pits, and \\
detect and map buried \\
waste materials and \\
other significant \\
features.
\end{tabular} & & $\begin{array}{l}\text { WHC-SD-EN- } \\
\text { TI-060 }\end{array}$ & \begin{tabular}{|l} 
Sandness, \\
GA
\end{tabular} & Apr-92 & 113 & No. & \\
\hline \begin{tabular}{|l|} 
Planned pipeline; \\
North of 300 Area
\end{tabular} & 300 & & $\mathrm{x}$ & & & & & & & & & & & $\begin{array}{l}\text { Determine if Indian } \\
\text { graves or other } \\
\text { artifacts are present. }\end{array}$ & $10 \mathrm{ft}$ & PNL memo & G. Sandness & 1992 & $\mathrm{NA}$ & $\mathrm{NA}$ & \\
\hline \begin{tabular}{|l|} 
Solid Waste Burial \\
Ground (Early \\
Burial Ground)
\end{tabular} & 300 & & \begin{tabular}{|l|} 
GSSI SIR \\
10 with \\
300-MHz \\
antenna
\end{tabular} & \begin{tabular}{|l|} 
Geonics \\
EM-31D
\end{tabular} & & & & & & & & & & $\begin{array}{l}\text { General reconnais- } \\
\text { sance to locate } \\
\text { concentrations of } \\
\text { buried waste. }\end{array}$ & $10-18^{\prime}$ & BHI-00212 & \begin{tabular}{|l|} 
Bergstrom \\
KA; Mitchell \\
TH; Bolin BJ
\end{tabular} & May-95 & 34 & No & \\
\hline $\begin{array}{l}\text { Undocumented Solid } \\
\text { Waste Burial } \\
\text { Ground }\end{array}$ & 300 & & \begin{tabular}{|l} 
GSSI SIR \\
10 with \\
$300-\mathrm{MHz}$ \\
antenna
\end{tabular} & \begin{tabular}{|l|} 
Geonics \\
EM-31D
\end{tabular} & & & & & & & & & & \begin{tabular}{l|} 
Determine extent of \\
the site and locate \\
possible trenches, pits, \\
and high concen- \\
trations of debris.
\end{tabular} & $10-18^{\prime}$ & BHI-00212 & \begin{tabular}{|l|} 
Bergstrom \\
KA; Mitchell \\
TH; Bolin BJ
\end{tabular} & May-95 & 34 & No & \\
\hline $\mid$ Fire Station & 300 & & $\mathrm{x}$ & & & & & & & & & $?$ & & 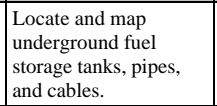 & $15 \mathrm{ft}$ & & G. Sandness & 1992 & NA & $\mathrm{NA}$ & \\
\hline $\begin{array}{l}3000-1,2,3,4,12 \\
\text { underground fuel } \\
\text { storage tanks }\end{array}$ & 3000 & & $\mathrm{x}$ & & & & & & & & & $\mathrm{x}$ & & \begin{tabular}{|l|} 
Determine location, \\
depth, orientation, and \\
size of each tank. Also \\
map pipes and cables.
\end{tabular} & $15 \mathrm{ft}$ & \begin{tabular}{|l|} 
PNL letter \\
report to Mark \\
Morton, WHO
\end{tabular} & G. Sandness & 06-Nov-89 & $\mathrm{NA}$ & NA & \\
\hline $\mid 3000-5,6$ & 3000 & & $\mathrm{x}$ & & & & & & & & & $\mathrm{x}$ & & \begin{tabular}{|l|} 
Locate and map under- \\
ground fuel storage \\
tanks, pipes, and cables.
\end{tabular} & $15 \mathrm{ft}$ & $\begin{array}{l}\begin{array}{l}\text { PNL letter } \\
\text { report to Ron } \\
\text { Shuck, WHO }\end{array} \\
\end{array}$ & G. Sandness & 16-Aug-89 & NA & NA & \\
\hline $3000-8,9,10,11$ & 3000 & & $\mathrm{x}$ & & & & & & & & & $\mathrm{X}$ & & \begin{tabular}{|l|}
$\begin{array}{l}\text { Locate and map } \\
\text { underground fuel } \\
\text { storage tanks, pipes, } \\
\text { and cables. }\end{array}$ \\
\end{tabular} & $15 \mathrm{ft}$ & $\begin{array}{l}\text { PNL letter } \\
\text { report to Ron } \\
\text { Shuck, WHO }\end{array}$ & G. Sandness & 16-Aug-89 & $\mathrm{NA}$ & NA & \\
\hline $\begin{array}{l}\text { 622-R Weather } \\
\text { Station }\end{array}$ & 600 & & $\mathrm{x}$ & & & & & & & & & $\mathrm{x}$ & & $\begin{array}{l}\text { Locate and map } \\
\text { underground fuel } \\
\text { storage tanks, pipes, } \\
\text { and cables. }\end{array}$ & $15 \mathrm{ft}$ & $\begin{array}{l}\text { PNL letter } \\
\text { report to Ron } \\
\text { Shuck, WHO }\end{array}$ & G. Sandness & 16-Aug-89 & $\mathrm{NA}$ & $\mathrm{NA}$ & \\
\hline
\end{tabular}




\begin{tabular}{|c|c|c|c|c|c|c|c|c|c|c|c|c|c|c|c|c|c|c|c|c|c|}
\hline \multirow[b]{2}{*}{ Site/Location } & \multirow[b]{2}{*}{ Area } & \multirow[b]{2}{*}{\begin{tabular}{|l|}
$\begin{array}{c}\text { Date(s) } \\
\text { of Survey }\end{array}$ \\
\end{tabular}} & \multicolumn{11}{|c|}{ Geophysical Methods Used } & \multirow[b]{2}{*}{ Survey Objectives } & \multirow[b]{2}{*}{$\begin{array}{c}\text { Max. } \\
\text { Depth } \\
\text { (ft) }\end{array}$} & \multirow[b]{2}{*}{$\begin{array}{c}\text { Reference } \\
\text { (Doc. No.) }\end{array}$} & \multirow[b]{2}{*}{ Author(s) } & \multirow[b]{2}{*}{ Date } & \multicolumn{2}{|c|}{ Bibliography } & \multirow[b]{2}{*}{ Comments } \\
\hline & & & GPR & $\mathrm{EM}$ & $\mathrm{IP}$ & $\begin{array}{c}\text { Downhole } \\
\text { Seismic }\end{array}$ & $\begin{array}{c}\begin{array}{c}\text { Seismic } \\
\text { (Acoustic) }\end{array} \\
\end{array}$ & Resistivity & ERT & Gravity & Magnetics & $\begin{array}{c}\text { Metal } \\
\text { Detector } \\
\end{array}$ & Other & & & & & & \begin{tabular}{|c|} 
Ref. \\
Number \\
\end{tabular} & Abstract? & \\
\hline 6652 L \& P; ALE & 600 & & $\mathrm{x}$ & & & & & & & & & $\mathrm{x}$ & & \begin{tabular}{|l} 
Locate and map \\
underground fuel \\
storage tanks pipes, \\
and cables.
\end{tabular} & $15 \mathrm{ft}$ & \begin{tabular}{|l} 
PNL letter \\
report to Mark \\
Morton, WHO
\end{tabular} & G. Sandness & 10-May-90 & NA & NA & \\
\hline Goose Egg Hill Area & 600 & & $\begin{array}{l}\text { GSSI SIR- } \\
8 \text { with 300- } \\
\text { MHz } \\
\text { antenna }\end{array}$ & $\begin{array}{l}\text { Geonics } \\
\text { EM-31 }\end{array}$ & & & & & & & & & & $\begin{array}{l}\text { Detect and map clastic } \\
\text { dikes. }\end{array}$ & & BHI-01103 & \begin{tabular}{|l|} 
Fecht, KR; \\
BN \\
Bjornstad, \\
DG Horton, \\
GV Last, SP \\
Reidel and \\
KA Lindsey \\
\end{tabular} & Jul-99 & 53 & Yes & \\
\hline \begin{tabular}{|l} 
H-06-H Military \\
Landfill, Wahluke \\
Slope
\end{tabular} & 600 & \begin{tabular}{|l|}
$7-27$ thruu \\
$8-4-92$
\end{tabular} & & \begin{tabular}{|l}
$\begin{array}{l}\text { Geonics } \\
\text { EM-31DL }\end{array}$ \\
\end{tabular} & & & & & & & \begin{tabular}{|l|} 
Scintrex \\
Magnetic \\
gradiometer \\
EDA Omni \\
Plus
\end{tabular} & & & $\begin{array}{l}\text { Locate and determine } \\
\text { the extent of waste site } \\
\text { at } 2 \text { areas. }\end{array}$ & & \begin{tabular}{|l|} 
WHC-SD-EN- \\
ER-001
\end{tabular} & \begin{tabular}{|l|}
$\begin{array}{l}\text { Gustafuson } \\
\text { F. W. }\end{array}$ \\
\end{tabular} & Dec-92 & 54 & No & \\
\hline \begin{tabular}{|l} 
H-83-L Military \\
Landfill, Wahluke \\
Slope
\end{tabular} & 600 & \begin{tabular}{|l|} 
7-27 thruu \\
$8-4-92$
\end{tabular} & & $\begin{array}{l}\begin{array}{l}\text { Geonics } \\
\text { EM-31DL }\end{array} \\
\end{array}$ & & & & & & & \begin{tabular}{|l|} 
Scintrex \\
Magnetic \\
gradiometer \\
EDA Onni \\
Plus
\end{tabular} & & & \begin{tabular}{|l} 
Locate and determine \\
the extent of waste site.
\end{tabular} & & \begin{tabular}{|l|}
$\begin{array}{l}\text { WHC-SD-EN- } \\
\text { ER-001 }\end{array}$ \\
\end{tabular} & \begin{tabular}{|l|} 
Gustafuson \\
F. W.
\end{tabular} & Dec-92 & 54 & No & \\
\hline Hanford Townsite & 600 & & & & & & $\mathrm{x}$ & & & & & & & $\begin{array}{l}\text { Detect buried stream } \\
\text { channels? }\end{array}$ & & $\mathrm{NA}$ & \begin{tabular}{|l|} 
Quenton \\
McDonald
\end{tabular} & ? & $\mathrm{NA}$ & NA & \\
\hline \begin{tabular}{|l} 
Non-Radioactive \\
Dangerous Waste \\
Landfill
\end{tabular} & 600 & & $x$ & & & & & & & & & & & $\begin{array}{l}\text { Locate } 20 \text { trenches, } \\
\text { determine thichness of } \\
\text { overlying fillil, and } \\
\text { locate primary } \\
\text { concentrations of } \\
\text { waste. }\end{array}$ & & $\begin{array}{l}\text { WHC-SD-EN- } \\
\text { TI-116 (1). } \\
\text { WHC-SA- } \\
\text { 2018/CONF- } \\
\text { 9181060-1 (2). } \\
\text { WHC-SD-EN- } \\
\text { DP-064 (3) }\end{array}$ & 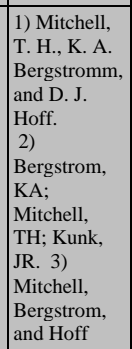 & \begin{tabular}{|l|} 
1) Jun-93 \\
2) Jul-93. \\
3) May-93
\end{tabular} & $\begin{array}{l}\text { 1) } 79 . \\
\text { 2) } 12 . \\
\text { 3) } 85\end{array}$ & $\begin{array}{l}\text { 1) No. } \\
\text { 2) Yes. } \\
\text { 3) No. }\end{array}$ & \\
\hline North Slope & 600 & & $x$ & & & & & & & & & & & & & $\begin{array}{l}\text { WHC-SD-EN- } \\
\text { TI-124 }\end{array}$ & \begin{tabular}{|l|}
$\begin{array}{l}\text { Bergstrom } \\
\text { and Mitchell }\end{array}$ \\
\end{tabular} & \begin{tabular}{|l|l|l} 
Jul-93 \\
\end{tabular} & 14 & No. & \\
\hline \begin{tabular}{|l}
$\begin{array}{l}\text { PSN-04 Military } \\
\text { Landfill, Wahluke } \\
\text { Slope }\end{array}$ \\
\end{tabular} & 600 & $\begin{array}{l}7-27 \text { thru } \\
88-92\end{array}$ & & \begin{tabular}{|l|l|}
$\begin{array}{l}\text { Geonics } \\
\text { EM-31DL }\end{array}$ \\
\end{tabular} & & & & & & & \begin{tabular}{|l|} 
Scintrex \\
Mangetic \\
gradiometer \\
EDA OOnmi \\
Plus
\end{tabular} & & & $\begin{array}{l}\text { Locate and determine } \\
\text { the extent tof waste site } \\
\text { at } 4 \text { areas. }\end{array}$ & & $\begin{array}{l}\text { WHC-SD-EN- } \\
\text { ER-001 }\end{array}$ & \begin{tabular}{|l|} 
Gustafuson \\
F. W.
\end{tabular} & Dec-92 & 54 & No & \\
\hline $\begin{array}{l}\text { Riverland Anti- } \\
\text { Aircraft Artillery } \\
\text { Site }\end{array}$ & 600 & & $\begin{array}{l}\text { GSSS SIR 8 } \\
\text { with-MHz } \\
\text { 300-MHz } \\
\text { antenna }\end{array}$ & & & & & & & & & & & $\begin{array}{l}\text { Determine if anything } \\
\text { was buried in three } \\
\text { myssterious dirt } \\
\text { mounds. }\end{array}$ & & $\begin{array}{l}\text { WHC-SD-EN- } \\
\text { TH-1117(1). } \\
\text { WHC-SD-EN- } \\
\text { DP-06- (2). } \\
\text { WHC-SA- } \\
\text { 2018/CONF- } \\
\text { 9310160-1 (3) }\end{array}$ & $\begin{array}{l}\text { 1) Berrgstrom } \\
\text { and Mitchll. } \\
\text { 2) Bergstrom } \\
\text { and Mitchell. } \\
\text { 3) } \\
\text { Bergstrom, } \\
\text { KA } \\
\text { Mitchell, TH } \\
\text { and Kunk, } \\
\text { JR }\end{array}$ & \begin{tabular}{|l} 
1) Apr- \\
1993. \\
2) 1992 3) \\
Jul-1993
\end{tabular} & $\begin{array}{l}\text { 1) } 8 \\
\text { 2) } 1 \\
\text { 3) } 12\end{array}$ & $\begin{array}{l}\text { 1) No } \\
\text { 2) No } \\
\text { 3) Yes }\end{array}$ & \\
\hline
\end{tabular}




\begin{tabular}{|c|c|c|c|c|c|c|c|c|c|c|c|c|c|c|c|c|c|c|c|c|c|}
\hline \multirow[b]{2}{*}{ Site/Location } & \multirow[b]{2}{*}{ Area } & \multirow[b]{2}{*}{$\begin{array}{c}\text { Date(s) } \\
\text { of Survey }\end{array}$} & \multicolumn{11}{|c|}{$\begin{array}{l}\text { Geophysical Methods Used } \\
\end{array}$} & \multirow[b]{2}{*}{ Survey Objectives } & \multirow[b]{2}{*}{$\begin{array}{l}\text { Max. } \\
\text { Depth } \\
\text { (ft) }\end{array}$} & \multirow[b]{2}{*}{$\begin{array}{l}\text { Reference } \\
\text { (Doc. No.) } \\
\end{array}$} & \multirow[b]{2}{*}{ Author(s) } & \multirow[b]{2}{*}{ Date } & \multicolumn{2}{|c|}{ Bibliography } & \multirow[b]{2}{*}{ Comments } \\
\hline & & & GPR & EM & IP & $\begin{array}{c}\text { Downhole } \\
\text { Seismic }\end{array}$ & $\begin{array}{c}\text { Seismic } \\
\text { (Acoustic) } \\
\end{array}$ & \begin{tabular}{|l|} 
Resistivity \\
\end{tabular} & ERT & Gravity & Magnetics & $\begin{array}{c}\text { Metal } \\
\text { Detector }\end{array}$ & Other & & & & & & \begin{tabular}{|c|} 
Ref. \\
Number
\end{tabular} & Abstract? & \\
\hline $\begin{array}{l}\text { Riverland Railroad } \\
\text { Maintenance Shop }\end{array}$ & 600 & & \begin{tabular}{|l|} 
GSSI SIR 8 \\
with \\
$300-\mathrm{MHz}$ \\
antenna
\end{tabular} & EM-31 & & & & & & & & & & $\begin{array}{l}\text { Locate buried } \\
\text { maintenance pits and } \\
\text { determine if } 12,000 \\
\text { gal. UST had been } \\
\text { removed during } \\
\text { decommissioning. }\end{array}$ & & \begin{tabular}{|l|} 
WHC-SD-EN- \\
TI-117 (1) \\
WHC-SDEN- \\
DP-061 (2) \\
\end{tabular} & \begin{tabular}{|l|} 
1) \\
Bergstrom, \\
KA and \\
Mitchell. \\
2) Bergstrom \\
and Mitchell \\
\end{tabular} & $\begin{array}{l}\text { 1) Apr-93 } \\
\text { 2) } 1992\end{array}$ & $\begin{array}{l}\text { 1) } 8 \\
\text { 2) } 1\end{array}$ & $\begin{array}{l}\text { 1) No. } \\
\text { 2) No. }\end{array}$ & \\
\hline $\begin{array}{l}\text { White Bluffs Crib, } \\
\text { White Bluffs } \\
\text { Townsite }\end{array}$ & 600 & & \begin{tabular}{|l|} 
GSSI SIR \\
10 with \\
500-MHz \\
antenna
\end{tabular} & \begin{tabular}{|l|} 
Geonics \\
EM-31DL
\end{tabular} & & & & & & & & \begin{tabular}{|l|} 
Metrotech \\
810 \\
Utility/ \\
Pipe \\
Locator \\
\end{tabular} & & \begin{tabular}{|l|} 
Locate pipes leading to \\
two cribs and analyze \\
distribution network \\
within cribs.
\end{tabular} & & $\begin{array}{l}\text { WHC-SD-EN- } \\
\text { ER-002 }\end{array}$ & \begin{tabular}{|l|} 
Westinghouse \\
Hanford \\
Company \\
(WHC)
\end{tabular} & Apr-92 & 118 & No. & \\
\hline |703-1 & 700 & & $\mathrm{x}$ & & & & & & & & & $\mathrm{x}$ & & \begin{tabular}{|l} 
Locate and map \\
underground fuel \\
storage tanks, pipes, \\
and cables.
\end{tabular} & $15 \mathrm{ft}$ & $\begin{array}{l}\text { PNL letter } \\
\text { report to Ron } \\
\text { Shuck, WHO }\end{array}$ & G. Sandness & |16-Aug-89 & NA & $\mathrm{NA}$ & \\
\hline $\begin{array}{l}\text { Hanford Site } \\
\text { General }\end{array}$ & $\mathrm{NA}$ & & \begin{tabular}{|l|} 
GSSI SIR 8 \\
and 10 with \\
$300-\mathrm{MHz}$ \\
antenna
\end{tabular} & & & & & & & & & & & \begin{tabular}{|l|} 
Interpret Ground- \\
Penetrating Radar data.
\end{tabular} & & \begin{tabular}{|l|} 
WHC-SA-2018, \\
CONF- \\
$9310160-1$
\end{tabular} & \begin{tabular}{|l} 
Bergstrom, \\
KA; \\
Mitchell, \\
TH; Kunk, \\
JR
\end{tabular} & Jul-93 & 12 & Yes & \\
\hline $\begin{array}{l}\text { Hanford Site } \\
\text { General }\end{array}$ & $\mathrm{NA}$ & & & & & & \begin{tabular}{|l} 
Shallow \\
High- \\
Resolution \\
Reflection
\end{tabular} & & & & & & & $\begin{array}{l}\text { Determine feasibility } \\
\text { of the Shallow High- } \\
\text { Resolution Seismic } \\
\text { Reflection Technique. }\end{array}$ & & $\begin{array}{l}\text { WHC-SD-EN- } \\
\text { TI-120 }\end{array}$ & \begin{tabular}{|l|} 
Narbutovskih, \\
SM
\end{tabular} & 30-Jul-93 & 97 & Yes & \\
\hline
\end{tabular}




\section{Appendix B}

Bibliography With Abstracts (where available) Regarding Shallow Surface Geophysical Surveys at the Hanford Site 
1. Bergstrom, K. A., and T. H. Mitchell. 1992. Data Package for Geophysical Investigations of Riverland ERA. WHC-SD-EN-DP-061, Westinghouse Hanford Company, Richland, Washington.

2. Bergstrom, K. A., and T. H. Mitchell. 1993. Ground Penetrating Radar Investigation for Proposed Borehole, South Pond, 100 N Area. WHC-SD-EN-TI-094, Westinghouse Hanford Company, Richland, Washington.

3. Bergstrom, K. A., and T. H. Mitchell. 1993. Ground Penetrating Radar Investigation for Proposed Borehole, 116-K-2, $100 \mathrm{~K}$ Area. WHC-SD-EN-TI-092, Westinghouse Hanford Company, Richland, Washington.

4. Bergstrom, K. A., and T. H. Mitchell. 1993. Ground Penetrating Radar Investigation for Proposed Borehole, 119-N, 100 N Area. WHC-SD-EN-TI-090, Westinghouse Hanford Company, Richland, Washington.

5. Bergstrom, K. A., and T. H. Mitchell. 1993. Ground Penetrating Radar Investigation for Proposed Borehole, 116-F-6, 100 F Area. WHC-SD-EN-TI-115, Westinghouse Hanford Company, Richland, Washington.

6. Bergstrom, K. A., and T. H. Mitchell. 1993. Ground Penetrating Radar Investigation for Proposed Borehole, 299-W15-218, 200 West Area. WHC-SD-EN-TI-096, Westinghouse Hanford Company, Richland, Washington.

7. Bergstrom, K. A., and T. H. Mitchell. 1993. Ground Penetrating Radar Investigation for Proposed Borehole, 116-N-2, 100 N Area. WHC-SD-EN-TI-088, Westinghouse Hanford Company, Richland, Washington.

8. Bergstrom, K. A., and T. H. Mitchell. 1993. Ground Penetrating Radar Investigation for Riverland ERA. WHC-SD-EN-TI-117, Rev. 0, Westinghouse Hanford Company, Richland, Washington.

9. Bergstrom, K. A., and T. H. Mitchell. 1993. Ground Penetrating Radar Investigation for Proposed Borehole, 116-F-9D, 100 F Area. WHC-SD-EN-TI-129, Westinghouse Hanford Company, Richland, Washington.

10. Bergstrom, K. A., and T. H. Mitchell. 1993. Geophysical Investigation of Trench 116-F-4, 100 F Area. WHC-SD-EN-TI-119, Rev. 0, Westinghouse Hanford Company, Richland, Washington.

11. Bergstrom, K. A., and T. H. Mitchell. 1993. Ground Penetrating Radar Investigation for Proposed Well Sites, 299-W15-219, 299-W15-220, 299-W8-252; 200 West Area. WHC-SD-ENTI-153, Westinghouse Hanford Company, Richland, Washington. 
12. Bergstrom, K. A., T. H. Mitchell, and J. R. Kunk. 1993. Interpretation of Ground Penetrating Radar data at the Hanford Site, Richland, Washington. WHC-SA-2018, CONF-9310160-1, Westinghouse Hanford Company, Richland, Washington. ABSTRACT: Ground-Penetrating Radar (GPR) is being used extensively during characterization and remediation of chemical and radioactive waste sites at the Hanford Site in Washington State. Time and money for GPR investigations are often not included during the planning and budgeting phase. Therefore, GPR investigations must be inexpensive and quick to minimize impact on already established budgets and schedules. An approach to survey design, data collection, and interpretation has been developed, which emphasizes speed and budget with minimal impact on the integrity of the interpretation or quality of the data. The following simple rules of thumb can be applied: 1) assemble as much pre-survey information as possible; 2) clearly define survey objectives prior to designing the survey and determine which combination of geophysical methods will best meet the objectives; 3 ) continuously communicate with the client, before, during and after the investigation; 4) only experienced GPR interpreters should acquire the field data; 5) use real-time monitoring of the data to determine where and how much data to collect and assist in the interpretation; 6) always "error" in favor of collecting too much data; 7) surveys should have closely spaced (preferably 5 feet, no more than 10 feet) orthogonal profiles; 8) when possible, pull the antenna by hand.

13. Bergstrom, K. A., and T. H. Mitchell. 1993. Geophysical Investigation for Proposed Test Pit 116-F-3 100F Area. WHC-SD-EN-TI-118, Westinghouse Hanford Company, Richland, Washington.

14. Bergstrom, K. A., and T. H. Mitchell. 1993. Ground Penetrating Radar Investigation for North Slope ERA. WHC-SD-EN-TI-124, Westinghouse Hanford Company, Richland, Washington.

15. Bergstrom, K. A., T. H. Mitchell, and J. P. Kiesler. 1993. Geophysical Investigation for the 116-C-2A Pluto Crib, 100 B/C Area. WHC-SD-EN-TI-154, Westinghouse Hanford Company, Richland, Washington.

16. Bergstrom, K. A., and T. H. Mitchell. 1993. Ground Penetrating Radar Investigation at 216U-4 and 216-U-16; 200 West Area. WHC-SD-EN-TI-176, Westinghouse Hanford Company, Richland, Washington.

17. Bergstrom, K. A., T. H. Mitchell, and A. L. Langstaff. 1993. Geophysical Investigation of the 118-B-1 Burial Grounds, 100-B/C Area, Hanford Site, Washington. WHC-SD-EN-TI-137, Rev. 0, Westinghouse Hanford Company, Richland, Washington.

18. Bergstrom, K. A., and T. H. Mitchell. 1993. Geophysical Investigation of the Sodium Dichromate Transfer Station, 100 D Area. WHC-SD-EN-TI-178, Rev. 0, Westinghouse Hanford Company, Richland, Washington.

19. Bergstrom, K. A., and T. H. Mitchell. 1993. Geophysical Investigation of the 118-B-1 Burial Ground, 100 B/C Area. WHC-SD-EN-TI-138, Westinghouse Hanford Company, Richland, Washington. 
20. Bergstrom, K. A. 1993. Geophysical Survey of 105-DR Pluto Crib, 116-DR-4, 100-D Area. WHC-SD-EN-TI-195, Westinghouse Hanford Company, Richland, Washington. ABSTRACT: The objective of this Geophysical Survey was to verify the location of the 105-DR Pluto Crib, 116DR-4. A surface monument currently marks its location. The crib is 10 feet by 10 feet and 15 feet deep. Ground-Penetrating Radar was the geophysical method selected to conduct the investigation.

21. Bergstrom, K. A., and T. H. Mitchell. 1994. Geophysical Survey for Proposed Borehole 199-K108A, 100-K Area. WHC-SD-EN-TI-228, Westinghouse Hanford Company, Richland, Washington. ABSTRACT: The objective of the electromagnetic survey was to locate subsurface obstructions that may affect the drilling of proposed borehole, 199-K-108A, $75 \mathrm{ft}$ southeast of the $105 \mathrm{KW}$ Building, 100-K Area. Based upon the results of the survey, possible drill sites within the zone, with the least likelihood of encountering identified obstructions, were identified.

22. Bergstrom, K. A., and T. H. Mitchell. 1994. Data Package for Geophysical Investigation of Burial Ground 118-B-1; 100 B/C Area. WHC-SD-EN-DP-066, Westinghouse Hanford Company, Richland, Washington.

23. Bergstrom, K. A., T. H. Mitchell, and G. J. Szwartz. 1994. Geophysical Investigation of the 118-H-2 Burial Ground, 100 H Area, Hanford Site, Washington. WHC-SD-EN-TI-208, Rev. 0, Westinghouse Hanford Company, Richland, Washington.

24. Bergstrom, K. A., T. H. Mitchell, and Kiesler. 1994. Geophysical Investigation of the 118-H-1 Burial Grounds, 100 H Area. WHC-SD-EN-TI-207, Rev. 0, Westinghouse Hanford Company, Richland, Washington.

25. Bergstrom, K. A., T. H. Mitchell, and G. J. Szwartz. 1994. Geophysical Investigation of the 1607-D3 Septic System, 100 D Area. WHC-SD-EN-TI-227, Rev. 0, Westinghouse Hanford Company, Richland, Washington.

26. Bergstrom, K. A. 1994. Geophysical investigation of the 'Thimble', 100-H Area. WHC-SDEN-TI-210, Westinghouse Hanford Company, Richland, Washington. ABSTRACT: This report summarizes the results of the geophysical investigations conducted as part of the characterization of the buried "Thimble" site. The site is located just south of the 116-H-2 Crib and is in the 100-HR-2 Operable Unit. Available documentation has located it between, and at the convergence of, two railroad spurs that run north-south. A concrete monument is believed to mark the site. The burial ground is suspected of containing a vertical safety rod thimble that is reportedly $40 \mathrm{ft}$ long. Ground-penetrating radar (GPR) and electromagnetic induction (EMI) were the two techniques used in the investigation. The methods were selected because they are non-intrusive, relatively fast, economical, and have been used successfully in other similar investigations on the Hanford Site. The objective of the investigation was to locate the buried thimble.

27. Bergstrom, K. A. 1994. Geophysical Investigation of the 216-B-3-1 Ditch, Operable Unit 200BP-11, 200 East Area. WHC-SD-EN-TI-281, Westinghouse Hanford Company, Richland, Washington. ABSTRACT: Ditch 216-B-3-1 is located within the 200-BP-11 Operable Unit, 
located immediately northeast of the 200 East Area. At one time, it drained into B Pond. The ditch has been filled with soil and the surrounding area reclaimed. There is no remaining physical evidence showing the original location of the ditch. Survey stakes were recently emplaced that show the documented location of the ditch from survey coordinates. The objective of this investigation was to verify the staked location of the ditch with non-intrusive geophysical methods. Ground-penetrating radar (GPR) and electromagnetic induction (EMI) were the methods selected for the investigation. GPR has been used successfully to locate similar ditches in other parts of the Hanford Reservation. EMI was used because it is much quicker to collect and interpret, and if successful, could be used to rapidly map the entire length of the ditch. Results are discussed.

28. Bergstrom, K. A. 1994. Geophysical Investigation of 216-U-8 Clay Vitrified Pipe Transfer Line, 200 West Area. WHC-SD-EN-TI-241, Westinghouse Hanford Company, Richland, Washington. ABSTRACT: Two geophysical surveys were conducted over a vitrified clay pipeline (VCP) that was used to transfer liquid radioactive waste from the 224-U Building to the 216-U-8 and 216-U-12 cribs. The objectives of the surveys were to locate the VCP in the northern site, locate the bends in the VCP in the southern site, and locate possible utilities or pipelines at both sites. Ground-penetrating radar (GPR) was the method chosen for the surveys. Electromagnetic induction (EMI) was also used at the southern site to map the extent of a possible pipeline. It is very difficult to detect most VCPs with GPR, however, excavation boundaries for the pipeline are often discernible. The VCP was not identified in the GPR data at the northern site. Its anticipated depth was 10-12 ft. The VCP at the southern site appears to be much shallower. The data suggests it may be $5 \mathrm{ft}$ or less below the surface in places. The edges of the excavation from N100 to N190 are between E120 and E135 and were quite distinct in the data. However, the excavation boundaries weren't apparent north of N190, suggesting that the VCP bends to the north near N200. Several profiles were extended beyond N200. These profiles detected a linear, with the characteristic signature of a pipeline, $\sim 3 \mathrm{ft}$ below the surface. There was no previous knowledge of the pipeline. The linear was traced across the entire surface contamination area with an EMI geophysical tool.

29. Bergstrom, K. A., and J. W. Fassett. 1994. Geophysical Investigations of French Drain 116-B-9, and Dry Well 116-B-10, 100 B/C Area. WHC-SD-EN-TI-213, Westinghouse Hanford Company, Richland, Washington. ABSTRACT: French Drain 116-B-9 and Dry Well 116-B-10 are both located within the $100 \mathrm{~B} / \mathrm{C}-2$ Operable Unit, $100 \mathrm{~B} / \mathrm{C}$ Area (Figure 1). The 116-B-9 French Drain is $\sim 4 \mathrm{ft}$ in diameter by 3-ft-deep. The exact location or use of the drain is not clear. The 116-B-10 Dry Well is a $3 \mathrm{ft}$-diameter, tile-lined well on a concrete slab, $7 \mathrm{ft}$ below the surface, overlain by a manhole cover (DOE-RL 1991). The exact location of the well is uncertain. The objective of the survey was to locate the Dry Well and the French Drain. The area to be investigated had several buildings in the area which subsequently have been torn down. Ground penetrating radar (GPR) was the geophysical method chosen for the investigation.

30. Bergstrom, K. A., and T. H. Mitchell. 1994. Data Package for Geophysical Studies at the 118-H-1, 118-H-2, 118-H-3, and Thimble Burial Grounds, 100 H Area. WHC-SD-EN-DP-069, Westinghouse Hanford Company, Richland, Washington. 
31. Bergstrom, K. A. 1994. Ground-Penetrating Radar Investigations Conducted in the 100 Areas, Hanford Site: Fiscal Year 1992. WHC-SD-EN-TI-204, Westinghouse Hanford Company, Richland, Washington. ABSTRACT: During Fiscal Year 1992, the Geophysics Group conducted forty-five Ground-Penetrating Radar (GPR) surveys in the 100 Areas (Figure 1). Objectives for the investigations varied, from locating cribs, trenches, and septic systems to helping site boreholes. The results of each investigation were delivered to clients in the form of a map that summarized the interpretation of a given site. No formal reports were prepared. The purpose of this document is to show where and why each of the surveys was conducted. The data and interpretation of each survey are available by contacting the Westinghouse Hanford Company, Geophysics Group. A map showing the location and basic parameters of each survey can be found in the Appendices of this report.

32. Bergstrom, K. A., T. H. Mitchell, and B. J. Bolin. 1995. Geophysical Investigation of the 120-KE-3 and 118-K-2 Sites, 100-KR-2 Operable Unit. BHI-00180-Rev. 0, Bechtel Hanford, Inc., Richland, Washington. ABSTRACT: Geophysical investigations using ground-penetrating radar (GPR) and electromagnetic induction (EMI) were conducted at two waste sites, 120-KE-3 and 118-K-2, in the 100-K Area. The 120-KE-3 waste site (Figure 2), also known as the 183-Filter Water Facility Trench and 100-KE-3, received sulfuric acid sludge from sulfuric acid storage tanks that were contaminated with $700 \mathrm{~kg}$ of mercury. Part or all of the trench was excavated when an outside contractor attempted to recover the mercury. The actual size of the 'disturbed area' from the trench and subsequent excavation is unknown. The objective of the geophysical investigation was to locate the original or reworked trench.

33. Bergstrom, K. A., T. H. Mitchell, and B. J. Bolin. 1995. Geophysical Investigations of the 618-7 Burial Ground, 300-FF-2 Operable Unit. BHI-00313, Rev. 0, Bechtel Hanford, Inc., Richland, Washington.

34. Bergstrom, K. A., T. H. Mitchell, and B. J. Bolin. 1995. Geophysical Investigations of the 316-4 (300 North Crib), the 618-8 Burial Ground, the 618-13 (Mound), the Undocumented Solid Waste Burial Ground, the Solid Waste Burial Ground (Early Burial Ground) and the Burial Trench West of the Process Trenches, 300-FF-2 Operable Unit. BHI-00212, Rev. O, Bechtel Hanford, Inc., Richland, Washington.

35. Bergstrom, K. A., and T. H. Mitchell. 1995. Geophysical Investigations of the 100-F-1 Depression, 100-F-14 Vent Pipe, PNL Parallel Pits, 100-FR-2 Operable Unit. BHI-00343, Rev. 0, Bechtel Hanford, Inc., Richland, Washington.

36. Bergstrom, K. A., and T. H. Mitchell. 1996. Geophysical Investigations of the 116-D-1A and 116-D-1B Liquid Waste Disposal Trenches, 100-D Area, 100-DR-1 Operable Unit. BHI-00396, Rev. 0, Bechtel Hanford, Inc., Richland, Washington. ABSTRACT: This report is a compilation of the results from four separate Ground-Penetrating Radar (GPR) investigations conducted over or near Liquid Waste Disposal Trenches 116-D-1A and 116-D-1B in the 100-D Area. The bulk of the data are from three investigations conducted between September 1991 and February 1992. The objectives, grid spacing, recording windows, and antennas varied with each 
investigation. These data were compiled and additional GPR data were collected to fill in gaps from the earlier surveys. This report integrates these data sets into a single interpretation.

37. Bergstrom, K. A., and T. H. Mitchell. 1996. Geophysical Investigation of the 116-B-11 Retention Basin, 116-B-1 Liquid Waste Disposal Trench, and 116-B-13 Sludge Trench, 100BC-1 Operable Unit. BHI-00717, Bechtel Hanford, Inc., Richland, Washington. ABSTRACT: This report summarizes the results of geophysical investigations and data integration conducted for the 116-B-1 liquid waste disposal trench, the 116-B-11 retention basin, and the 116-B-13 sludge trench, which are all located at the 100-BC-1 Operable Unit (Figure 1). The objectives of this investigation include the following: map the extent of 116-B-1 trench and any anomalous debris/ material that may have been buried in it; locate and accurately map the walls and pipelines associated with the 116-B-11 retention basin; and locate and map the 116-B-13 sludge trench.

38. Bergstrom, K. A., and T. H. Mitchell. 1996. Geophysical Investigation of the 116-C-5 Retention Basin 100-BC-1, Operable Unit. BHI-00716, Bechtel Hanford, Inc., Richland, Washington. ABSTRACT: This report provides a summary of the results of geophysical investigations and data integration conducted for the 116-C-5 Retention Basin. The retention basin and the surrounding terrain have changed significantly since they were in operation. Numerous piles of soil and debris, that appear to be related to the removal of the walls, are found throughout the site. The objectives of this investigation include: locate and accurately map the perimeter of the two retention basins; locate and accurately map any unknown features (e.g., major concentration of debris, pipelines, and utilities) that could be a factor during subsurface remediation work; and determine optimum locations for cone penetrometers and test pits that are to be used to assess the presence of subsurface contamination.

39. Bergstrom, K. A., and T. H. Mitchell. 1996. Geophysical Investigation of the 116-H-1 Liquid Waste Disposal Trench, 100-HR-1 Operable Unit. BHI-00715, Bechtel Hanford, Inc., Richland, Washington. ABSTRACT: A geophysical investigation and data integration were conducted for the 116-H-1 Liquid Waste Disposal Trench, which is located in the 100-HR-1 Operable Unit. The 116-H-1 Liquid Waste Disposal Trench is also known as the 107-H Liquid Waste Disposal Trench, the 107-H Rupture Effluent Trench, and the 107-H Trench (Deford and Einan 1995). The trench was primarily used to hold effluent from the 107-H Retention Basin that had become radioactive from contact with ruptured fuel elements. The effluent may include debris from the ruptured fuel elements (Koop 1964). The 116-H-1 Liquid Waste Disposal Trench was also used to hold water and sludge from the 107-H Retention Basin during the basin's deactivation in 1965.

40. Bergstrom, K. A., and T. H. Mitchell. 1996. Geophysical Investigations in the Group II Sites 100-D Area. BHI-00786, Bechtel Hanford, Inc., Richland, Washington. ABSTRACT: This document contains the results of a geophysical investigation of nine Group II sites: 1) 116-D-7 and 116-DR-9 Retention Basins; 2) 116-DR- 1 and 116-DR-2 Liquid Effluent Trenches; 3) 107-D-1, 107-D-2, 107-D-3, 107-D-4, and 107-D-5 Sludge trenches. All nine sites were directly associated with the operation of the retention basins which are located in the 100-DR-1 Operable Unit. An area south of 116-D-7 was also investigated as an area that may have contained buried debris. The objectives of this investigation include the following: 1) locate, map, and/or verify locations of 
subsurface pipelines and utilities; 2) locate and map five sludge disposal trenches; 3) map the extent of 116-DR-1 and 116-DR-2 liquid disposal trenches; 4) locate and accurately map any unknown features (e.g., major concentration of debris, pipelines, and utilities) that could be a factor during subsurface remediation work.

41. Bergstrom, K. A. 1996. Geophysical Investigation of the 116-DR-6 Liquid Waste Disposal Trench and the 132-DR-1 Waste Water Pumping Station, 100D Area. WHC-SD-EN-TI-222, Rev. 0, Westinghouse Hanford Company, Richland, Washington. ABSTRACT: Groundpenetrating radar investigations were conducted at the covered-over locations of the 116-DR-6 Liquid Waste Disposal Trench and the 132-DR-1 Waste Water Pumping Station to better define the actual subsurface locations of these facilities and to locate any piping that may pass into them. These surveys were only partially successful.

42. Bergstrom, K. A., D. J. Bolin, and T. H. Mitchell. 1997. Geophysical Investigation of the 61810 and 618-11 Burial Grounds, 300-FF-2 Operable Unit. BHI-00291-Rev. 1, Bechtel Hanford, Inc., Richland, Washington. ABSTRACT: This document summarizes the results of geophysical investigations conducted at two radioactive solid waste burial grounds, 618-10 and 618-11. The burial grounds are located $\sim 4.5$ miles and 7 miles north of the 300 Area, respectively. These sites are within the 300-FF-2 Operable Unit, where geophysical techniques are being used to characterize the distribution of solid waste in the subsurface as part of the Limited Field Investigations for this operable unit.

43. Bergstrom, K. A., T. H. Mitchell, J. J. Sharp, and K. M. Singleton. 1998. ERC Geophysical Investigation Summary, Building 221-U, 200 West Area. BHI-01182, Bechtel Hanford Inc., Richland, Washington. ABSTRACT: This report summarizes the geophysical investigation and survey of the 221- U Plant, southern side of building. The effective depth of investigation was 0 to $14 \mathrm{ft}$ throughout most of the survey area. The overall data quality was very good. Numerous pipelines and utilities were detected. Some correlated with available drawings while others could not be related to a drawing. Table 1 summarizes each GPR linear that was detected. Throughout the northern half of the site, scattered subsurface anomalies and anomalous zones (high concentration of anomalies) were detected. Most range in depth from 1- to 6- $\mathrm{ft}$ and have the characteristics of anthropogenic features. Due to the proximity of the site to the 221-U Building, it is anticipated that the majority of the anomalies are construction debris, possibly related to the construction of the plant. However, there is no direct evidence of their true origin. A buried tank is believed to be located in the vicinity of N570/E150. Available drawings show that it is roughly $25 \mathrm{ft}$ below grade. Although the tank was not detected with GPR, the approximate location of the tank was estimated from circumstantial evidence (i.e., surface features, subsurface pipe locations). Final interpretation maps were made for the site. The depths to each linear, anomaly, and anomalous zone are shown on the interpretation maps.

44. Berven, B. A., J. E. Nyquist, M. S. Blair, C. A. Little, and R. B. Gammage. 1989. Automation of Geophysical Surveys Used in Assessment of Hazardous Waste. CONF-891053-2, Oak Ridge National Laboratory, Tennessee. ABSTRACT: Geophysical surveys generally involve noninvasive measurements made from the ground surface to assess subterranean physical formations. 
These surveys can serve to locate subsurface contamination or assist in understanding the potential movement of these materials in groundwater. Oak Ridge National Laboratory has recently merged two technologies to automate geophysical measurements. The terrain conductivity meter (Geonics EM-31) measures subsurface conductivity detecting anomalies such as water-filled trenches or buried water or gas lines. The Ultra Sonic Ranging and Data System was developed to locate a surveyor on a specified area using triangulation with ultrasonic time-of-flight positioning. The surveyor's position is transmitted once a second to a microcomputer in the field. Simultaneously, the measurement taken by the surveyor's portable instrument is also transmitted to the microcomputer using a radio frequency link. Up to 3,600 paired measurements and locations can be stored directly in a microcomputer for analysis each hour. This represents a significant advance over conventional techniques by providing more data with fewer errors (related to transcription of information) in which the data can be analyzed in the field. Output from the analysis includes report-ready tables, two-dimensional contour plots, and three-dimensional mesh plots. With these plots, areas of high subsurface conductivity can be readily visualized while in the field so that any additional data needed can be obtained sooner with less cost.

45. Clark, S. W. 1992. Geophysical Surveys at the Horn Rapids Landfill. WHC-SD-EN-TI-015, Rev. 0, Westinghouse Hanford Company, Richland, Washington.

46. Clayton, E. A. 1993. Integrated Test Plan for Demonstration of Surface Geophysics at the VOC-Arid ID Host Site. WHC-SD-EN-AP-116, Rev. 0, Westinghouse Hanford Company, Richland, Washington.

47. Clayton, E. A., and R. E. Lewis. 1995. Data Fusion Qualitative Sensitivity Analysis. PNL10393, Battelle Pacific Northwest Laboratories, Richland, Washington. ABSTRACT: Data fusion is the mathematical combination (or fusion) of disparate data sets into a single interpretation. The data fusion software developed by CRC was initially demonstrated on a data set collected at the Hanford Site where three types of data were combined. These data were 1) seismic reflection, 2) seismic refraction, and 3) depth to geologic horizons. The fused results included a contour map of the top of a low-permeability horizon. This report discusses the results of a sensitivity analysis of data fusion software to variations in its input parameters.

48. Collins, H. D., R. P. Gribble, T. E. Hall, and W. M. Lechelt. 1995. Ultra Wide Band Radar Holographic Imaging of Buried Waste at DOE Sites. PNL-SA-25908, CONF-950450-12, Battelle Pacific Northwest Laboratories, Richland, Washington. ABSTRACT: Ultra wideband linear array holography is a unique real-time imaging technique for in situ inspection of buried waste at various DOE sites. The array can be mounted on various platforms such as crane booms, pickup trucks, ATVs, and scanned generating '3-D' subsurface images in real time. Inspection speeds are 0.5 to 2 meters/sec, if the image is viewed in real time, greater for off-line processing. The Ground Penetrating Holographic (GPH) system developed for inspection of DOE sites employs two 32-element arrays of tapered-slot antenna operating at 5-GHz and $2.5-\mathrm{GHz}$ center frequencies. The GPH system, which is mounted on a small trailer with a computer image processor, display, and power supply, is capable of imaging a wide swath (1 to 2 meters) with its linear arrays. The lower frequency array will be used at INEL (for greater depth penetration) because of high soil 
attenuation. Recent holographic '3-D' images of buried waste container lids and dielectrics obtained in Hanford sand and INEL soils at various depths graphically illustrate the unique image resolution capabilities of the system. Experimental results using the 5-GHz array will be presented showing the excellent holographic image quality of various subsurface targets in sand and INEL soil.

49. Drnevich, V. P., R. Slgado, A. Ashmawy, W. P. Grant, and P. Vallenas. 1995. Interpretation of Large-Strain Geophysical Crosshole Tests. WHC-SA-2959, CONF-9511128-14, Westinghouse Hanford Company, Richland, Washington. ABSTRACT: A seismic crosshole test has been developed where large dynamic forces are applied in a borehole. This paper provides an improved, systematic interpretation scheme for the data from these large-strain geophysical crosshole tests. Use is made of both the measured velocities at each sensor and the travel times. The measured velocity at each sensor location is shown to be a good measure of the soil particle velocity at that location. Travel times to specific features on the velocity time history, such as first crossover, are used to generate travel time curves for the waves which are nonlinear. At some distance the amplitudes reduce to where the stress-strain behavior is essentially linear and independent of strain amplitude. This fact is used together with the measurements at the three sensor locations in a rational approach for fitting curves of shear wave velocity versus distance from the source hole that allow the determination of the shear wave velocity and the shear strain amplitude at each of the sensor locations as well as the shear wave velocity associated with small-strain (linear) behavior.

50. Elbring, G. J., and S. M. Narbutovskih. 1994. Integrated Test Plan for Crosswell Compressional and Shear Wave Seismic Tomography for Site Characterization at the VOC Arid Site. WHC-SD-EN-TP-039, Westinghouse Hanford Company, Richland, Washington.

ABSTRACT: This integrated test plan describes the demonstration of the crosswell acoustic tomography technique as part of the Volatile Organic Compounds-Arid Integrated Demonstration (VOC-Arid ID). The purpose of this demonstration is to image the subsurface seismic velocity structure and to relate the resulting velocity model to lithology and saturation. In fiscal year (FY) 1994 an initial fielding will test three different downhole sources at two different sites at the Hanford U.S. Department of Energy facility to identify which sources will provide the energy required to propagate between existing steel-cased wells at these two sites. Once this has been established, a second fielding will perform a full compressional and shear wave tomographic survey at the most favorable site. Data reduction, analysis, and interpretation of this full data set will be completed by the end of this fiscal year. Data collection for a second survey will be completed by the end of the fiscal year, and data reduction for this data set will be completed in FY 1995. The specific need is detailed subsurface characterization with minimum intrusion. This technique also has applications for long term vadose zone monitoring for both Resource Conservation and Recovery Act (RCRA) waste storage facilities and for remediation monitoring. Images produced are continuous between boreholes. This is a significant improvement over the single point data derived solely from core information. Saturation changes, either naturally occurring (e.g., perched water tables) or remediation-induced (e.g., water table mounding from injection wells or during inwell air sparging), could be imaged. These crosswell data allow optimal borehole placement for groundwater remediation, associated monitoring wells, and possibly evaluation of the effective influence of a particular remediation technique. 
51. Elbring, G. J. 1995. Comparison of Lower-Frequency (<1000 Hz) Downhole Seismic Sources for Use at Environmental Sites. SAND-95-0344C, CONF-950450-3, Sandia National Laboratory, Albuquerque, New Mexico. ABSTRACT: In conjunction with crosswell seismic surveying being done at the Hanford Site in south-central Washington, four different downhole seismic sources have been tested between the same set of boreholes. The four sources evaluated were the Bolt airgun, the OYO-Conoco orbital vibrator, and two Sandia-developed vertical vibrators, one pneumatically driven and the other based on a magnetostrictive actuator. The sources generate seismic energy in the lower frequency range of less than $1000 \mathrm{~Hz}$ and have different frequency characteristics, radiation patterns, energy levels, and operational considerations. A collection of identical data sets with all four sources allows the direct comparison of these characteristics and an evaluation of the suitability of each source for a given site and target.

52. Fassett, J., and K. A. Bergstrom. 1996. Geophysical Investigation of the 216-U-1/2 Pipeline, 200 West Area. WHC-SD-EN-TI-262, Westinghouse Hanford Company, Richland, Washington. ABSTRACT: Ground-penetrating radar was used at three locations in an attempt to locate and determine the depth of the $216-\mathrm{U}-1 / 2$ pipeline. Many anomalies were found, all very useful to the project, but only some of which were identified with the pipeline.

53. Fecht, K. R., B. N. Bjornstad, D. G. Horton, G. V. Last, S. P. Reidel, and K. A. Lindsey. 1999. Clastic Injection Dikes of the Pasco Basin and Vicinity. BHI-01103, Rev. 0, Bechtel Hanford, Inc., Richland, Washington. ABSTRACT: The atlas provides a summary of the current understanding of clastic injection dikes in the Pasco Basin and vicinity. The atlas is a compilation of photographs, maps, tables, illustrations, and text that provides a summary of the physical description, emplacement history and mechanisms, and hydrologic characteristics of clastic injection dikes. The information that has been assembled is based on geologic, geophysical, and geohydrologic investigations conducted in the Pasco Basin and vicinity. This atlas includes a description of the test results of surface-based, nonintrusive geophysical surveys over buried clastic injection dikes. The test methods used were ground-penetrating radar and electromagnetic induction.

54. Gustafson, F. W. 1992. Geophysical Surveys of Military Landfills Located on Hanford's Wahluke (North) Slope-In Support of the Wahluke Slope Expedited Response Action. WHCSD-EN-ER-001, Westinghouse Hanford Company, Richland, Washington.

55. Hoekstra, P., J. Vandergraft, M. Blohm, and D. Porter. 1993. Geophysical Data Fusion for Subsurface Imaging. Phase 1. DOE/MC/29106-3531, Coleman Research Corp., Columbia, Maryland. ABSTRACT: A geophysical data fusion methodology is under development to combine data from complementary geophysical sensors and incorporate geophysical understanding to obtain three-dimensional images of the subsurface. The research reported here is the first phase of a three-phase project. The project focuses on the characterization of thin clay lenses (aquitards) in a highly stratified sand and clay coastal geology to depths of up to 300 feet. The sensor suite used in this work includes time-domain electromagnetic induction (TDEM) and near-surface seismic techniques. During this first phase of the project, enhancements to the acquisition and processing of TDEM data were studied, by use of simulated data, to assess improvements for the detection of thin clay layers. Secondly, studies were made of the use of compressional wave and 
shear wave seismic reflection data by using state-of-the-art, high-frequency vibrator technology. Finally, a newly developed processing technique, called "data fusion," was implemented to process the geophysical data, and to incorporate a mathematical model of the subsurface strata. Examples are given of the results when applied to real seismic data collected at Hanford, Washington, and for simulated data based on the geology of the Savannah River Site.

56. Johnson, V. M., K. A. Lindsey, J. R. Serne, R. S. Edrington, and T. H. Mitchell. 1995. The N-Springs Barrier Wall Drilling Program Data Package. BHI-00135-Rev. 1, Bechtel Hanford, Inc., Richland, Washington.

57. Kiesler, J. P., and T. H. Mitchell. 1993. Ground Penetrating Radar Investigation Over Leaking Pipe at U-Plant Cut; 200 West Area. WHC-SD-EN-TI-180, Westinghouse Hanford Company, Richland, Washington.

58. Kiesler, J. P., K. A. Bergstrom, and T. H. Mitchell. 1993. Geophysical Investigation of 218-W$4 C$ Trenches \#1 and \#4, 200 West. WHC-SD-EN-TI-174, Westinghouse Hanford Company, Richland, Washington. ABSTRACT: The objective of these surveys was to determine the effectiveness of ground-penetrating radar (GPR) for defining the depth and location of barrels in Burial Ground 218-W-4C, 200 West area of the Hanford Reservation. GPR data were collected in a portion of trench (number sign) 1-module 3 and trench (number sign) 4. The GPR system used for this work utilized a 300-megahertz antenna to transmit the electromagnetic energy into the ground. A percentage of the transmitted energy is reflected back to a receiving antenna where variations in the return signal are recorded. Common reflectors include natural geologic conditions such as bedding, cementation moisture and clay, or man-made objects such as pipes, barrels, foundations, and buried wires. Depth of penetration, which varies with changes in geology, ranged from 10 to 15 feet for this survey. The method is limited in depth by transmit power, receiver sensitivity, and attenuation of the transmitted energy. Depth of investigation is also limited by highly conductive materials, such as metal drums, which reflect all the transmitted energy, preventing investigation below such objects. Display and interpretation of the data are similar to that of seismic reflection data. In some areas interpretations can be straightforward; however, unknown parameters within a highly variable subsurface often yield complex data.

59. Kiesler, J. P. 1994. Geophysical Investigation for Proposed Flow Meter Installation Holes, 100H Area. WHC-SD-EN-TI-263, Westinghouse Hanford Company, Richland, Washington. ABSTRACT: The objectives of the surveys were to locate subsurface obstructions that may affect the drilling of two holes to be fit with flow meters in the 100-H Area of the Hanford Site (Figure 1). Possible drill sites with the least likelihood of encountering identified obstructions were identified based upon the results of the survey. Ground-penetrating radar (GPR) was the method selected for the investigations. The electromagnetic induction method was also used to verify that the general site is relatively void of metallic debris at depth.

60. Kiesler, J. P. 1994. Geophysical Investigation of Trench 4, Burial Ground 218-W-4C, 200 West Area. WHC-SD-EN-TI-285, Westinghouse Hanford Company, Richland, Washington. ABSTRACT: This report contains the results of a geophysical investigation conducted to 
characterize Trench 4, located in Burial Ground 218-W-4C, 200 West Area. Trench 4 is where transuranic (TRU) waste is stored. The primary objective of these geophysical investigations was to determine the outer edges of the trench/modules and select locations for plate-bearing tests. The test locations are to be 5 to $8 \mathrm{ft}$. beyond the edges of the trench. Secondary objectives include differentiating between the different types of waste containers within a given trench, determining the amount of soil cover over the waste containers, and locating the module boundaries. Groundpenetrating radar (GPR) and electromagnetic induction (EMI) were the methods selected for this investigation.

61. Kiesler, J. P. 1996. Geophysical Investigation of Selected Sites in Burial Grounds 218-W-3A, 4B, and -4C. WHC-SD-EN-TI-295, Westinghouse Hanford Company, Richland, Washington.

62. Kunk, J. R., and T. H. Mitchell. 1991. Geophysical Surveys at the 618-9 Burial Ground. WHC-MR-0271, Westinghouse Hanford Company, Richland, Washington.

63. Kunk, J. R., S. M. Narbutovskih, K. A. Bergstrom, and T. H. Mitchell. 1993. Phase I Summary of Surface Geophysical Studies in the 300-FF-5 Operable Unit. WHC-SD-EN-TI069, Westinghouse Hanford Company, Richland, Washington.

64. Kunk, J. R. 1993. Data Package for Geophysical Studies in the 300-FF-5 Operable Unit. WHC-SD-EN-DP-059, Westinghouse Hanford Company, Richland, Washington.

65. Kunk, J. R., and K. A. Bergstrom. 1994. Geophysical Investigation of the 116-DR-8 Crib, 100 D Area. WHC-SD-EN-TI-225, Rev. 0, Westinghouse Hanford Company, Richland, Washington.

66. Lewis, R. E., and S. S. Teel. 1994. A Survey of Existing and Emerging Technologies for External Detection of Liquid Leaks at the Hanford Site. PNL-10176, Pacific Northwest Laboratory, Richland, Washington.

67. Mitchell, T. H., and J. R. Kunk. 1991. Geophysical Surveys at the 1100-EM-1 South Pit. WHC-MR-0243, Westinghouse Hanford Company, Richland, Washington.

68. Mitchell, T. H, and K. A. Bergstrom. 1992. Geophysical Investigation at the Sodium Dichromate Barrel Landfill. WHC-SD-EN-ES-30, Westinghouse Hanford Company, Richland, Washington.

69. Mitchell, T. H., and J. R. Kunk. 1992? 100-DR-1 Geophysical Surveys. WHC-MR-0257, Westinghouse Hanford Company, Richland, Washington.

70. Mitchell, T. H., and J. R. Kunk. 1992? 100-HR-1 Geophysical Surveys. WHC-MR-0263, Westinghouse Hanford Company, Richland, Washington. 
71. Mitchell, T. H., and K. A. Bergstrom. 1993. Ground Penetrating Radar Investigation for Proposed Borehole, 1322-N, 100 N Area. WHC-SD-EN-TI-089, Westinghouse Hanford Company, Richland, Washington.

72. Mitchell, T. H., and K. A. Bergstrom. 1993. Data Package for Geophysical Investigations of Sodium Dichromate Barrel Landfill ERA. WHC-SD-EN-DP-061, Westinghouse Hanford Company, Richland, Washington.

73. Mitchell, T. H., and K. A. Bergstrom. 1993. Ground Penetrating Radar Investigation for Proposed Borehole, 116-KW-4A, $100 \mathrm{~K}$ Area. WHC-SD-EN-TI-093, Westinghouse Hanford Company, Richland, Washington.

74. Mitchell, T. H., and K. A. Bergstrom. 1993. Ground Penetrating Radar Investigation for Proposed Borehole, 116-KW-3A, $100 \mathrm{~K}$ Area. WHC-SD-EN-TI-095, Westinghouse Hanford Company, Richland, Washington.

75. Mitchell, T. H., and K. A. Bergstrom. 1993. Ground Penetrating Radar Investigation for Proposed Borehole, UN-100-N-17, 100 N Area. WHC-SD-EN-TI-091, Westinghouse Hanford Company, Richland, Washington.

76. Mitchell, T. H., J. R. Kunk, and K. A. Bergstrom. 1993. Ground Penetrating Radar Investigation for Proposed Borehole, 116-F-2, 100 F Area. WHC-SD-EN-TI-114, Westinghouse Hanford Company, Richland, Washington.

77. Mitchell, T. H., K. A. Bergstrom, and D. J. Hoff. 1993. Data Package for Geophysical Investigation of the Nonradioactive Dangerous Waste Landfill (NRDWL). WHC-SD-EN-DP064, Westinghouse Hanford Company, Richland, Washington.

78. Mitchell, T. H., and K. A. Bergstrom. 1993. Ground Penetrating Radar Investigation for Proposed Borehole, 116-F-14, 100 F Area. WHC-SD-EN-TI-126, Westinghouse Hanford Company, Richland, Washington.

79. Mitchell, T. H., K. A. Bergstrom, and D. J. Hoff. 1993. Geophysical Investigation of the Nonradioactive Dangerous Waste Landfill (NRDWL). WHC-SD-EN-TI-116, Westinghouse Hanford Company, Richland, Washington.

80. Mitchell, T. H., and K. A. Bergstrom. 1993. Ground Penetrating Radar Investigation for Proposed Wells, 299-W15-223 and 299-W15-25, 200 West Area. WHC-SD-EN-TI-196, Westinghouse Hanford Company, Richland, Washington.

81. Mitchell, T. H., and K. A. Bergstrom. 1993. Ground Penetrating Radar Investigation at the 116-DR-3 Burial Grounds; 100 D Area. WHC-SD-EN-TI-177, Westinghouse Hanford Company, Richland, Washington. 
82. Mitchell, T. H. 1993. Geophysical Survey for Cone Penetrometer Site, CPT-4, 200 West Area. WHC-SD-EN-TI-162, Westinghouse Hanford Company, Richland, Washington.

ABSTRACT: This report describes a geophysical survey performed at the Hanford Reservation. The objective of the survey was to locate subsurface obstructions that may affect cone penetrometer work at site CPT-4, adjacent to and west of borehole 299-W18-252, Figure 1. Based upon the results of the survey, possible "drill sites" within the zone, with the least likelihood of encountering identified obstructions, were identified.

83. Mitchell, T. H., and K. A. Bergstrom. 1994. Geophysical Investigation of 116-DR-7, $100 \mathrm{D}$ Area. WHC-SD-EN-TI-179, Rev. 0, Westinghouse Hanford Company, Richland, Washington.

84. Mitchell, T. H., and K. A. Bergstrom. 1994. Ground Penetrating Radar Investigation for Proposed Borehole, 299-W11-232, 200 West Area. WHC-SD-EN-TI-141, Westinghouse Hanford Company, Richland, Washington.

85. Mitchell, T. H., and K. A. Bergstrom. 1994. Data Package for Geophysical Investigation of Burial Ground 118-C-1; 100 B/C Area. WHC-SD-EN-DP-067, Westinghouse Hanford Company, Richland, Washington.

86. Mitchell, T. H., K. A. Bergstrom, and G. J. Szwartz. 1994. Geophysical Investigation of the 118-H-3 Burial Grounds, 100 H Area. WHC-SD-EN-TI-209, Rev. 0, Westinghouse Hanford Company, Richland, Washington.

87. Mitchell, T. H., and K. A. Bergstrom. 1994. Ground Penetrating Radar Investigation for Cone Penetrometer Survey: CPT-BT-23; 200 West Area. WHC-SD-EN-TI-193, Westinghouse Hanford Company, Richland, Washington.

88. Mitchell, T. H., and K. A. Bergstrom. 1994. Geophysical Investigation of the 118-D-5 Ball 3X Burial Ground, 100-D Area. WHC-SD-EN-TI-226, Westinghouse Hanford Company, Richland, Washington.

89. Mitchell, T. H. 1994. Geophysical Survey for Proposed Borehole 199-K-106A, 100-K Area. WHC-SD-EN-TI-230, Westinghouse Hanford Company, Richland, Washington.

ABSTRACT: The objective of the survey was to locate subsurface obstructions that may affect the drilling of proposed borehole, 199-K-106A, about $50 \mathrm{ft}$ east of the 1,714 KW Building, 100-K Area. Based upon the results of the survey, possible drill sites within the zone-with the least likelihood of encountering identified obstructions-were identified. The ground-penetrating radar (GPR) system used for this work utilized a $300-\mathrm{MHz}$ antenna to transmit the electromagnetic (EM) energy into the ground. The transmitted energy is reflected back to a receiving antenna where variations in the return signal are recorded. Common reflectors include natural geologic conditions such as bedding, cementation, moisture, and clay or man-made objects such as pipes, barrels, foundations, and buried wires. The method is limited in depth by transmit power, receiver sensitivity, frequency, and attenuation of the transmitted energy, which can be strongly affected by geology. Depth of investigation is also influenced by highly conductive material, such as metal 
drums, which reflect all the energy back to the receiver. Therefore, the method cannot "see" below such objects. Maximum depth of penetration for this survey seemed to be about 10 to $12 \mathrm{ft}$.

90. Mitchell, T. H. 1994. Geophysical Investigations in the 100 Areas: Fiscal Year 1991 Through December 1993. WHC-SD-EN-TI-247, Westinghouse Hanford Company, Richland, Washington. ABSTRACT: The geophysical investigations identified in this document were conducted by the Westinghouse Hanford Company (WHC) Surface Geophysics Team, Geophysics Group between October 1991 and December 1993. The investigations supported 100-Area activities for the Resource Conservation and Recovery Act of 1976 (RCRA) and the Comprehensive Environmental Response, Compensations and Liability Act of 1980 (CERCLA). The primary intent of this document is to provide a general map location and the associated document number for investigations that have been conducted as of December 1993. The results of the individual investigations are not included here. The results of all of these investigations have been previously reported individually in WHC supporting documents. The investigations conducted during Fiscal Year (FY) 1992 are summarized in a single WHC document, WHC-SD-EN-TI-204 Rev. 0. A brief summary of some of the successful applications of geophysics in the 100-Areas is included.

91. Mitchell, T. H. 1994. Geophysical Survey for Proposed Borehole 199-K-107A, 100-K Area. WHC-SD-EN-TI-229, Westinghouse Hanford Company, Richland, Washington.

ABSTRACT: The objective of the survey was to locate subsurface obstructions that may affect the drilling of proposed borehole, 199-K-107A, located about $100 \mathrm{ft}$ northwest of the $105 \mathrm{KW}$ Building, 100-K Area. Based upon the results of the survey, possible drill sites within the zone, with the least likelihood of encountering identified obstructions, were identified. The ground-penetrating radar (GPR) system used for this work utilized a 300-megahertz antenna to transmit the electromagnetic (EM) energy into the ground. The transmitted energy is reflected back to a receiving antenna where variations in the return signal are recorded. Common reflectors include natural geologic conditions such as bedding, cementation, moisture, and clay or man-made objects such as pipes, barrels, foundations, and buried wires. Several isolated anomalies, at various depths, are observed in the data. Additionally, two areas that appear disturbed, with perplexing character, are plotted. Because of the uncertain nature of these two areas, they were avoided when recommending a borehole location. Initially, the proposed borehole was staked at N130/E122. The newly proposed borehole location is N139/E176. This location appears free of anomalies and is over $10 \mathrm{ft}$ from interpreted linear anomalies/pipe-like features.

92. Mitchell, T. H. 1995. Geophysical Surveys for Proposed Boreholes 299-W15-25, 26 and 27, 200 West Area. WHC-SD-EN-TI-282, Westinghouse Hanford Company, Richland, Washington. ABSTRACT: The objective of the survey was to locate subsurface obstructions that may affect the drilling of the proposed boreholes 299-W15-25, 299-W15-26, and 299-W15-27, north of the 321-Z building. The possible drill sites within the zone with the least likelihood of encountering identified obstructions were identified based on the results of the survey. Ground-penetrating radar (GPR) was the geophysical method chosen for the shallow characterization of this site. 
93. Mitchell, T. H., and K. A. Bergstrom. 1995. Geophysical Investigations of the 116-B-3 Pluto Crib, 116-B-4 French Drain, 116-B-5 Crib, 100-BC-1 Operable Unit. BHI-00344, Rev. 0, Bechtel Hanford Inc., Richland, Washington.

94. Mitchell, T. H. 1995. Geophysical Survey for Proposed Boreholes, 199-K-109A, 199-K-110A, and 199-K-111A, 100K Area. WHC-SD-EN-TI-253, Westinghouse Hanford Company, Richland, Washington. ABSTRACT: A survey was conducted to locate subsurface obstructions that may affect the drilling of three proposed boreholes in the $100 \mathrm{~K}$ Area. Drill sites with the least likelihood of encountering obstructions were identified by the method of ground-penetrating radar. These results are presented in this document.

95. Mitchell, T. H. 1995. Geophysical Investigation of the SQ Tanks, 221-T Building, 200 West Area. WHC-SD-EN-TI-223, Westinghouse Hanford Company, Richland, Washington. ABSTRACT: The SQ Tanks are located on the northwest side of the 221-T plant, in the 200-West Area of the Hanford Reservation. The objective of the survey was to locate subsurface obstructions that may affect the removal of tanks and supporting structures. Based upon the results of the survey, obstructions and linears were identified. Access was limited in the survey area due to structures, buildings, and construction work.

96. Moore, B. A. 1982. Geophysical Investigations of the Gable Mountain Pond-West Lake Area, Hanford Site, South-Central Washington. A thesis for Master of Science in Hydrology at University of Idaho. RHO-SA-239, Rockwell Hanford Operations, Richland, Washington. ABSTRACT: Low-level radioactive liquid waste is discharged directly to cribs, trenches, and unlined surface ponds at the U.S. Department of Energy's Hanford Site, Washington. One such facility, Gable Mountain Pond, recharges the unconfined aquifer contained within glaciofluvial sediments of the Hanford formation. The underlying Elephant Mountain basalt separates the unconfined aquifer from a confined aquifer in the Rattlesnake Ridge interbed. The hydraulic head of the unconfined aquifer is greater than that of the confined aquifer, creating the potential for downward flow of groundwater to the confined aquifer.

A two-phase research project was designed to investigate radionuclide contamination of the Rattlesnake Ridge interbed. Hydrogeologic studies focused on characterizing the aquifer, developing a conceptual groundwater flow model, and evaluating the extent of aquifer contamination. Surface geophysical methods were employed to delineate the geologic controls for potential groundwater movement from Gable Mountain Pond to the Rattlesnake Ridge interbed. Structures and erosional features were mapped on the basalt surface, the lower boundary of the unconfined aquifer, using gravity, electrical resistivity, and magnetic techniques. The geophysical surveys extended information gained at drilling sites to interwell areas. The results of the geophysical investigation are presented in the report.

Two structures were inferred at the basalt surface by correlating gravity and geoelectric trends to stratigraphic trends. These structures include a syncline and an anticline. The syncline trends northwest, paralleling the Western Gable Mountain anticline, which forms a basalt topographic ridge to the north. Magnetic and geoelectric trends suggest secondary erosion of the syncline. The northwest-trending anticline, located to the south of Gable Mountain Pond, is partially eroded at the crest, as indicated by well data. 
Trends in geoelectric and gravity profiles indicate that negative anomalies are spatially associated with erosional lows and synclines. Positive anomalies correspond to erosional highs and anticlines. In general, magnetic profile trends do not correspond to known near-surface basalt features.

Magnetic trends and well data indicate that the Elephant Mountain basalt is completely eroded at various locations within the study area. The Rattlesnake Ridge interbed, the first interbed below the Elephant Mountain basalt, is assumed to be in direct contact with the unconfined aquifer at these localities. Minor areal erosion of the Elephant Mountain basalt is evident throughout the study area. Thinning of this confining layer, in conjunction with the presence of fractures and cooling joints within the basalt, may create possible avenues for aquifer intercommunication.

97. Narbutovskih, S. M. 1993. Feasibility of the Shallow High Resolution Seismic Reflection Technique for Use at the Hanford Site. WHC-SD-EN-TI-120, Westinghouse Hanford Company, Richland, Washington. ABSTRACT: Data obtained during site characterization should be useful to assess the need for remediation, to evaluate and design effective remedial plans, and to allow long-term monitoring to discern remediation effectiveness. A valuable environmental tool that incorporates this data is a model that describes groundwater, vadose zone flow, and transport characteristics. Data on geology and hydrology combined with information on contaminant sources are incorporated into these conceptual models that delineate the relative significance of the various fluid migration pathways. Downstream these same models also support risk assessment, remediation design, and long-term assessment of remediation effectiveness. Consequently, the building of coherent, accurate vadose zone and groundwater models is fundamental to a successful remediation. Among the important requirements for these models is accurate knowledge of flow domain boundaries and soil characteristics. At the Hanford Site, this knowledge is obtained primarily from borehole data, which provides information only at a point. In the high energy flood and fluvial deposits found at the Hanford Site, it can, at times, be difficult to correlate lithologic horizons between boreholes. Where there is no borehole control, our understanding of the geometry of hydrogeologic boundaries - and thus of fluid migration paths - is limited. Surface geophysical techniques are generally used to provide a measure of geologic control between boreholes. In particular, the seismic reflection method has the potential to provide the greatest resolution of the subsurface hydrogeology between and beyond boreholes.

98. Narbutovskih, S. M., and F. Michelsen. 1994. Preliminary Results of a Seismic Borehole Test Using Downhole Shaped Charges at the DOE Hanford Site. WHC-SA-2304, CONF-940353-4, Westinghouse Hanford Company, Richland, Washington. ABSTRACT: Geophysical site characterization studies can be important steps in the process of designing and monitoring remediation at hazardous waste storage facilities. Use of seismic techniques for subsurface characterization at the DOE Hanford Site has been limited. One reason is the lack of borehole velocity control, and low-velocity sediments are highly attenuative. Consequently, standard techniques to provide velocity control are not adequate. Both Vertical Seismic Profiling and reversed VSP surveys are currently being investigated to provide velocity control and for subsurface imaging capabilities. Recently, a jet perforating gun was used to perforate a double-cased borehole in the 200 West Area. Acoustic emissions were recorded from numerous depths to obtain velocity control for a previous surface survey conducted in the same area. Both P- and S-wave data were recorded simultaneously 
from multiple horizons using the DAS-1 seismograph and 3-component geophones. The data were analyzed for a variety of uses besides velocity control. Signal attenuation was studied as a function of source depth and offset distance to evaluate formation absorption while vertical resolution was determined from the frequency spectrum. Preliminary results indicate that adequate P-wave velocity control can be obtained even though the near-surface sediments are very attenuative. However, we conclude that the perforating gun produces little SH energy. Preliminary velocities indicate that reflection coefficients should be great enough to use surface techniques. Results from the frequency study suggest that a swept source for both surface and borehole surveys may be necessary to obtain required resolutions. Finally, signal attenuation as a function of formation facies suggest that seismic techniques may be useful in mapping perched water zones and for long-term vadose zone monitoring.

99. Narbutovskih, S. M. 1994. Integrated Test Plan for a Shallow High Resolution Compressional Seismic Reflection Demonstration. WHC-SD-EN-TP-048, Westinghouse Hanford Company, Richland, Washington. ABSTRACT: This integrated test plan describes the demonstration of a surface high-resolution seismic reflection acquisition system using swept source technology. Compressional wave data will be collected along a previously occupied seismic line associated with a recent seismic survey north of the 300 Area. The swept source system will be employed testing two very different high-resolution vibrator sources, one with a frequency range from 10 to $500 \mathrm{~Hz}$ and a smaller unit with a range from 20 to $1,500 \mathrm{~Hz}$. This will enable a precursory comparison of two vibrator data sets with standard impulse data. The data will be evaluated for the presence of reflected energy, signal strength, frequency content, and signal-to-noise ratio. If the water table can be distinguished from the Hanford/Ringold formation contact, then the high permeability Hanfordfilled channels can be mapped. Next, if details on the configuration of the Ringold middle mud can be discerned, this will allow detecting fluid pathway through the mud and confirm the depositional nature of this unit. Finally, by mapping the extent of the lower confining mud unit, areas where the polluted unconfined and lower confined aquifers communicate might be located. Another source and acquisition method will also be tested by gathering data along the same seismic line. This system uses a lightweight source that produces a high-velocity shock wave that strikes the earth's surface causing an acoustic wave to propagate downward. The acquisition method is nonconventional and is reported to eliminate obstructing noise such as groundroll and air blast. It is not expected that this system will have the imaging ability of the vibratory systems. However, it could prove to be economical for shallow applications when only compressional energy is needed.

100. Narbutovskih, S. M., F. B. Michelsen, J. C. Clark, and E. W. Christensen. 1995. High Resolution Seismic Reflection Test at the DOE Hanford Site. WHC-SA-2784, CONF-950450-6, Westinghouse Hanford Company, Richland, Washington. ABSTRACT: A recent test was conducted to ascertain the benefits of swept source technology for use at the DOE Hanford Site. Previous high-resolution seismic surveys suffered from coherent noise interference, poor signal transmission, and lack of borehole velocity control. P-wave data were collected with the T-2500 Minivib produced by IVI, Inc. and Oyo Geospace's DAS-1 acquisition system. Results showed a significant increase in signal-to-noise ratio, increased resolving power, and better depth penetration 
of the signal. It is concluded that swept source technology as part of a total systems approach significantly expands the capabilities of the shallow high-resolution seismic reflection method for use at the DOE Hanford Site.

101. Narbutovskih, S. M., T. D. Halter, M. D. Sweeney, W. Daily, and A. L. Ramirez. 1996. Electrical Resistivity Tomography at the DOE Hanford Site. WHC-SA-3035-FP, CONF960477-3, Westinghouse Hanford Company, Richland, Washington. ABSTRACT: Recent work at the DOE Hanford site has established the potential of applying Electrical Resistivity Tomography (ERT) for early leak detection under hazardous waste storage facilities. Several studies have been concluded to test the capabilities and limitations of ERT for two different applications. First, field experiments have been conducted to determine the utility of ERT to detect and map leaks from underground storage tanks during waste removal processes. Second, the use of ERT for long-term vadose zone monitoring has been tested under different field conditions of depth, installation design, acquisition mode/equipment, and infiltration chemistry. This work involves transferring the technology from Lawrence Livermore National Laboratory (LLNL) to the Resource Conservation and Recovery Act (RCRA) program at the DOE Hanford Site. This paper covers field training studies relevant to the second application for long-term vadose zone monitoring.

102. Narbutovskih, S. M. 1996. Electrical Resistivity Tomography for Early Vadose Leak Detection Under Single Shell Storage Tanks. WHC-SD-EN-TP-057, Fluor Daniel Hanford Inc., Richland, Washington. ABSTRACT: This document describes planned testing with Electrical Resistivity Tomography (ERT). It is prepared in support of TTP RL46WT51 Rev. 1, funded by the Tank Focus Area through the Office of Technology Integration. The primary goal of the testing for fiscal year 1996 (FY96) is to develop and demonstrate the ability to place vertical electrode arrays (VEA) with the cone penetrometer technology (CPT) to depths below existing single-shell tanks (SST) at the DOE Hanford Site. It is desirable to have the capability to use CPT for this application for obvious reasons. First, current methods of emplacement, drilled boreholes, are expensive with respect to the rest of the ERT operation. Cone penetrometer VEA emplacements offer the opportunity to significantly reduce installation costs. Second, use of CPT will reduce emplacement time from weeks or months to just several days depending on the number of VEAs and the depth of placement. ERT is preferable to other monitoring methods because operation costs and turn-around time are less than the current baselines of either groundwater sampling networks or borehole logging techniques. ERT cost savings can be substantial and will continue into the future. ERT can also provide complete coverage under a tank or other facility, which is an important supplement to existing monitoring methods. Groundwater sampling provides one data point per well and borehole logging provides data along a line in the ground. Neither provide information from beneath a facility and, thus, are not able to locate release points. These electrode arrays are used to acquire subsurface electrical resistance data in a manner appropriate for tomographic inversion. The resulting tomograms can then be used to detect, monitor, and track contaminated moisture plumes leaking from underground storage tanks during waste retrieval operations. 
103. Phillips, S. J., and J. R. Raymond. 1975. Monitoring and Characterization of Radionuclide Transport in the Hydrogeologic System. BNWLSA5494/CONF-750967-15, Pacific Northwest Laboratory, Richland, Washington. ABSTRACT: The groundwater monitoring program provides information and data on groundwater quality required to evaluate the impact of waste disposal practices on the Hanford Reservation. The program includes: collection and analysis of groundwater samples on a routine basis; data processing, analysis, and reporting; design, construction, and maintenance of well sampling structures; and design and implementation of supporting research studies. Within the overall framework of the Groundwater Monitoring Program, the 300 Area and Wye Burial Ground Characterization Program was initiated to evaluate transport of radionuclides in the partially saturated zone above the water table and to provide site characterization at solid waste burial locations on the Reservation. Methods for collecting and analyzing program data include geophysical exploration by ground-penetrating radar, refraction and reflection acoustics, magnetics, and metal detection; stratigraphic investigations by drilling and sample collection techniques; evaluation of transport phenomena by in situ psychrometric and gamma-neutron techniques; laboratory characterization of fluid and vapor transport-controlling mechanisms; and evaluation of biological radionuclide transport by organisms inhabiting contaminated areas.

104. Phillips, S. J., and J. R. Raymond. 1975. Monitoring and Characterization of Radionuclide Transport in the Hydrogeologic System. BNWLSA5494/CONF-750967-16, Battelle Pacific Northwest Laboratories, Richland, Washington. ABSTRACT: Historical records pertaining to the 300 North and Wye Burial Grounds at the Hanford Reservation were reviewed as a prerequisite to determining programs for land reclamation. All available historical documents, agency communications, and engineering drawings related to the study areas were located, reviewed, and analyzed. An inventory of recorded location, type, and quantity of radionuclides and associated materials in each burial ground was completed and distributed to cooperating investigators. A geophysical survey of the 300 North Burial Ground was conducted as a basis for detecting the composition, size, distribution, and depth of buried objects and characterizing the sediments in which they are buried. Acoustic, radar, magnetic, and metal detection surveys were completed and their applicability evaluated; drilling techniques and equipment for recovering and characterizing sediments and radioactive contaminated material were developed. Drilling will also determine the amount and dimensional extent of radionuclide migration; sediment-fluid interaction and fluid migration through the unsaturated zone at the 300 North Burial Ground were characterized. A study to determine biological transport of radionuclides at the Wye Burial Ground was also initiated. This study involved a preliminary survey of present flora and fauna inhabiting the Wye Burial Ground site. Plant tissue was chemically and radiochemically analyzed and investigated to determine radionuclide migration, possible dose effects, and population dynamics of burrowing animals that could potentially be exposed to buried waste materials.

105. Phillips, S. J., A. E. Reisenauer, W. H. Rickard, and G. A. Sandness. 1977. Initial Site Characterization and Evaluation of Radionuclide Contaminated Solid Waste Burial Grounds. BNWL-2184, Battelle Pacific Northwest Laboratories, Richland, Washington. 
106. Phillips, S. J., L. L. Ames, R. E. Fitzner, G. W. Gee, and G. A. Sandness. 1980. Characterization of the Hanford 300 Area Burial Grounds. Final Report: Decontamination and Decommissioning. PNL-2557, Battelle Pacific Northwest Laboratories, Richland, Washington.

ABSTRACT: Pacific Northwest Laboratory conducted a series of investigations at the Hanford Site to develop technologies for characterizing and monitoring radioactive waste burial facilities that could be used in determining appropriate decommissioning alternatives. Specific objectives were to develop unique functional geophysics, geochemical, soil physics, numerical modeling, and biological methodologies needed to better characterize and monitor buried radioactive waste disposal sites. To meet these objectives the project was divided into four tasks: Task I, Geophysical Evaluation - Geophysical surveys were taken to locate and define the gross composition of waste materials. Task II, Geochemical Analysis - The interaction of disposed radionuclides with geologic media was analyzed through an integrated radiochemical procedure. Task III, Fluid Transport and Modeling - Computer modeling of water migration in partially saturated groundwater systems was verified with actual data collected at a field test facility used to monitor micrometeorological and geohydrological energy and mass transfer factors. Task IV, Biological Transport -

Several biological organisms were evaluated for potential radionuclide uptake and transport. Along with the four tasks, the project included a review of pertinent literature and regulatory issues that might affect the alternatives selected. Surveys were taken of the surrounding area and specific sites and operations. The overall results indicated that the 300 Area Burial Grounds have been adequate in containing radioactive waste. Based on the results of the project, the alternatives identified for decommissioning these sites are exhumation and translocation, entombment, perpetual care, and abandonment. Perpetual care (currently used) appears to be the best decommissioning alternative for these burial grounds at this time. However, another alternative may be selected depending on future waste management policies, plans, or activities.

107. Ramirez, A., W. Daily, A. Binley, and D. LaBrecque. 1996. Tank Leak Detection Using Electrical Resistance Methods. UCRL-JC-122875, CONF-960477-4, Lawrence Livermore National Laboratory, California. ABSTRACT: Large volumes of hazardous liquids and highlevel radioactive wastes are stored worldwide in surface and underground tanks. Frequently, these tanks are found to leak, thereby resulting in not only a loss of stored inventory, but in contamination to soils and groundwater. It is important to develop a reliable method of detecting leaks before large quantities are emitted into the environment surrounding the tanks. Two field experiments were performed to evaluate the performance of electrical resistance tomography (ERT) as a leak detection method under metal underground storage tanks (UST). This paper provides a summary of the field experiments performed under a 15-m diameter steel tank mockup located at the Hanford Reservation.

108. Raymond, J. R. 1955. Applicability of Geophysical Methods of Exploration at Hanford Atomic Products Operation. HW38707XAB, General Electric Company, Richland, Washington, Hanford Atomic Products Operation. ABSTRACT: The applicability of electrical, gravitational, magnetic, seismic, and radiometric logging methods to the determination of the geological conditions at Hanford are evaluated for definition or delimitation of the basalt bedrock surface, the surface of the Ringold formation, and the groundwater table. The induction equipment, gravimeter, 
dip needle, magnetometer, and seismographs can be used to obtain bedrock information. The seismic techniques are the only geophysical methods by which the groundwater table depth and thickness of the aquifer can be obtained at this site.

109. Rohay, V. J., K. J. Swett, V. M. Johnson, G. V. Last, D. C. Lanigan, and L. A. Doremus. 1993. FY 93 Site Characterization Status Report and Data Package for the Carbon Tetrachloride Site. WHC-SD-EN-TI-202, Westinghouse Hanford Company, Richland, Washington. ABSTRACT: This report provides the status and accomplishments from fiscal year site characterization activities conducted as part of the 200 West Area Carbon Tetrachloride Expedited Response Action and the Volatile Organic Compounds - Arid Integrated Demonstration. The report includes, or references, all available raw data collected as part of these tasks. During fiscal year 1993, the 200 West Area Carbon Tetrachloride Expedited Response Action and the Volatile Organic Compounds, Arid Integrated Demonstration programs focused on the carbon tetrachloride plume in the unsaturated zone underlying the 200 West Area at the Hanford Site in southeast Washington.

110. Sandness, G. A. 1991. Report on Geological Surveys in the 300-FF-1 Operable Unit. EMO1032, Battelle Pacific Northwest Laboratories, Richland, Washington. ABSTRACT: This report describes a set of geophysical surveys performed by the Pacific Northwest Laboratory at selected locations within the 300-FF-1 Operable Unit at Hanford. Field work and preliminary data processing activities were initiated in September 1989. These actions were terminated by the Westinghouse Hanford Company before completion in December 1989. Work was reinitiated in October 1990, to complete the processing of the data that had already been collected and to report the results. Because the field work was only partially completed, the task objectives, as presented in the Statement of Work, could not be fully met. This report is, therefore, a progress report covering the work performed through December 11, 1989. This task involved 1) ground-penetrating radar surveys of the 618-4 and 618-5 Burial Grounds and 2) ground-penetrating radar and electromagnetic induction surveys along the assumed routes of the abandoned process sewers and radioactive liquid waste sewers in the 300-FF-1 Operable Unit. The surveys in the burial grounds were intended to identify burial trenches and pits to determine the depth of fill and to locate waste materials, including any that might be outside the perimeter fences. The surveys along the sewer routes were intended, first, to confirm the locations of the sewers as shown on existing maps or to otherwise accurately determine their locations, and second, to attempt to identify locations of possible leaks. 3 refs., 3 figs., 2 tabs.

111. Sandness, G. A. 1991. Geophysical Surveys Performed by the Automatic and Measurement Sciences Department of the Pacific Northwest National Laboratory at Hanford Burial Grounds 618-4 and 618-5. WHC-SD-EN-TI-061, Rev. 0, Westinghouse Hanford Company, Richland, Washington.

112. Sandness, G. A. 1991. Geophysical Surveys. WHC-SD-EN-TI-062, Rev. 0, Westinghouse Hanford Company, Richland, Washington. 
113. Sandness, G. A. 1992. Geophysical Surveys Performed by the Automatic and Measurement Sciences Department of the Pacific Northwest National Laboratory. WHC-SD-EN-TI-060, Rev. 0, Westinghouse Hanford Company, Richland, Washington.

114. Strait, S. R., and B. A. Moore. 1982. Geohydrology of the Rattlesnake Ridge Interbed in the Gable Mountain Pond Area. RHO-ST-38, Rockwell Hanford Operations, Richland, Washington. ABSTRACT: Liquid waste disposal practices at the U.S. Department of Energy's Hanford Site include the discharge of low-level radioactive liquid waste to cribs, trenches, and surface ponds. A study was conducted to develop a conceptual groundwater flow model and characterize the current distribution of radionuclides in the groundwater of the Rattlesnake Ridge interbed at one of these facilities, the Gable Mountain Pond (facility 216-A-25). Specially constructed wells were drilled in the vicinity of Gable Mountain Pond to obtain radionuclide distribution data and hydrologic properties of the aquifer. The acquired data were analyzed to determine concentrations of anions, cations, selected radionuclides and groundwater flow patterns. Geologic mechanisms, which may be responsible for the determined concentration distribution, were evaluated using borehole and subsurface geophysical techniques.

115. Sweeney, M. D. 1999. Environmental Monitoring Alternatives for the Liquid Effluent Retention Facility. PNNL-12024, Pacific Northwest National Laboratory, Richland, Washington.

116. Szwartz, G. J. 1994. Geophysical Investigation of Burn Pit, 128-H-1, 100-H Area. WHC-SDEN-TI-217, Westinghouse Hanford Company, Richland, Washington. ABSTRACT: The 128H-1 burn pit is located in the northeast corner of 100-H Area. The objective of the survey was to delineate subsurface features in the 128-H-1 burn pit that may affect the emplacement of soil-gas probes. Ground-penetrating radar (GPR) and electromagnetic induction (EMI) were the two techniques used in the investigation. The methods were selected because they are non-intrusive, relatively fast, economical, and have been used successfully in other geophysical investigations on the Hanford Site. The GPR system used for this work utilized a 300-MHz antenna to transmit the Em energy into the ground. The transmitted energy is reflected back to a receiving antenna where variations in the return signal are recorded. Common reflectors include natural geologic conditions such as bedding, cementation, moisture, and clay or man-made objects such as pipes, barrels, foundations, and buried wires. The studied depth, which varies from site to site, was 0 to $11 \mathrm{ft}$ for this survey. The method is limited in depth by transmit power, receiver sensitivity, and attenuation of the transmitted energy. Depth of investigation is influenced by highly conductive material, such as metal drums, which reflect all the energy back to the receiver. Therefore, the method cannot "see" below such objects.

117. U.S. Department of Energy (DOE). 1995. 118-B-1 Burial Ground Excavation Treatability Test Report. DOE/RL-95-34, Rev. 0, U.S. Department of Energy, Richland, Washington. ABSTRACT: This treatability investigation focused on the feasibility of excavating, analytical screening, and handling waste materials from the 118-B-1 Burial Ground located in the $100 \mathrm{~B} / \mathrm{C}$ Area of the Hanford Site. The 118-B-1 Burial Ground consists of $\sim 24$ trenches on a 7-acre parcel. Solid low-level radioactive wastes and other debris and trash associated with reactor operations were disposed in 28 burial grounds in the 100 Area between 1944 and 1973. The majority of waste 
generated from routine reactor operations was placed in seven primary burial grounds, including 118-B-1. The 118-B-1 Burial Ground was selected as the location to perform this treatability test based on the availability of historical data for this site, and because it was thought to be representative of other primary-use burial grounds in the 100 Area. Geophysical surveys were conducted over the burial ground to map the concentrations of waste and aid in the selection of test pit excavation locations. The test plan developed for this study integrated the Streamlined Approach for Environmental Restoration (SAFER), a U.S. Department of Energy (DOE) initiative based on both the Data Quality Objective (DQO) process and the observational approach. This treatability test is the first one at the Hanford Site to use the SAFER approach. The purpose of this study was 1) to support development of the Proposed Plan and Record of Decision, which would identify the approach to be used for burial ground remediation and 2) to provide specific engineering information for receiving waste generated from the 100 Area removal actions. The results of the treatability test can be used to determine the feasibility of performing excavation, analytical screening, and handling of burial ground materials from similar burial grounds.

118. Westinghouse Hanford Company (WHC). 1992. Geophysical Survey of White Bluffs Cribs Site. WHC-SD-EN-ER-002, Rev. 0, Prepared by IT Corporation, Irvine California, for Westinghouse Hanford Company, Richland, Washington.

119. Westinghouse Hanford Company (WHC). 1994. Columbia River Effluent Pipeline Survey. WHC-SD-EN-TI-278, Rev. 0, Prepared by Golder Associates Inc., for Westinghouse Hanford Company, Richland, Washington. 


\section{Appendix C}

Bibliography With Abstracts (where available) Regarding Borehole Geophysics at the Hanford Site 
1. Additon, M. K., K. R. Fecht, T. L. Jones, and G. V. Last. 1978. Scintillation Probe Profiles 200 East Area Crib Monitoring Wells. RHO-LD-28, Rockwell Hanford Company, Richland, Washington. ABSTRACT: Scintillation probe profiles from 154 monitoring wells located in 200 East Area have been compiled for input into the Long-Term Management of Low-Level Waste Project's database. A thallium-activated sodium iodide scintillation system and the scintillation equipment and instrumentation used to log these monitoring wells are described. In addition, waste management applications of scintillation probe profiles are discussed including the determination of the distribution of gamma-emitting radiocontaminants and the identification of stratigraphic units. Cross references for well number and associated crib facility designation are given in the appendices.

2. Additon, M. K., K. R. Fecht, T. L. Jones, and G. V. Last. 1978. Scintillation Probe Profiles 200 West Area Crib Monitoring Wells. RHO-LD-29, Rockwell Hanford Company, Richland, Washington. ABSTRACT: Scintillation probe profiles from 187 monitoring wells located in 200 West Area have been compiled for input into the Long-Term Management of Low-Level Waste Project's database. A thallium-activated sodium iodide scintillation system and the scintillation equipment and instrumentation used to log these monitoring wells are described. In addition, waste management applications of scintillation probe profiles are discussed, including the determination of the distribution of gamma-emitting radiocontaminants and the identification of stratigraphic units. Cross references for well number and associated crib facility designation are given in the appendices.

3. Arthur, R. J. 1990. 1990 Yearly Calibration of Pacific Northwest Laboratory's Gross-Gamma Borehole Geophysical Logging System. PNL-7460, Battelle Pacific Northwest Laboratories, Richland, Washington. ABSTRACT: This report describes the 1990 yearly calibration of a gross-gamma geophysical pulse-logging system owned by the U.S. Department of Energy (DOE) and operated by Pacific Northwest Laboratory (PNL). The calibration was conducted to permit the continued use of this system for geological and hydrologic studies associated with remedial investigation at the Hanford Site. Primary calibrations to equivalent uranium units were conducted in borehole model standards that were recently moved to the Hanford Site from the DOE field calibration facility in Spokane, Washington. The calibrations were performed in borehole models $\mathrm{SBL} / \mathrm{SBH}$ and SBA/SBB, which contain low equivalent-uranium concentrations. The integrity of the system throughout the previous year from gamma-ray monitoring was demonstrated using the before- and after-logging field calibration readings with the field source in calibration Positions 1 and 2. Most of the Position 1 readings are within an $8 \%$ limit that is set by the governing PNL technical reference procedure as a critical value above which the instrument is considered suspect. Many of the Position 2 readings exceed the $8 \%$ limit; however, the fluctuation was traced to fieldsource geometry variability that affected Position 1 count rates by up to 6\% and Position 2 count rates by as much as $16 \%$. Correlations were established based on two similar approaches for relating observed count rate in before- and after-logging field calibrations to equivalent uranium concentrations. The temperature drift of the gamma-ray probe was documented and amounts to less than $0.1 \% /{ }^{\circ} \mathrm{C}$ within the temperature range $0^{\circ} \mathrm{C}$ to $42^{\circ} \mathrm{C}$. The low-energy cutoff for the gross gamma-ray probe was determined to be between 46.5 and $59.5 \mathrm{keV}$. 
4. Arthur, R. J. 1991. 1991 Yearly Calibration of Pacific Northwest Laboratory's Gross GammaRay Borehole Geophysical Logging System. PNL-7785, Battelle Pacific Northwest Laboratories, Richland, Washington. ABSTRACT: This report describes the 1991 yearly calibration of a gross gamma-ray geophysical pulse-logging system owned by the U.S. Department of Energy (DOE) and operated by the Pacific Northwest Laboratory. The calibration was conducted to permit the continued use of this system for geologic and hydrologic studies associated with remedial investigations at the Hanford Site. Primary calibrations to equivalent uranium units were conducted in DOE borehole model standards that reside on the Hanford Site. The calibrations were performed in borehole models SBL/SBH and SBA/SBB, which contain low-equivalent uranium concentrations. Correlations were established based on two similar approaches for relating observed count rate in before- and after-logging field calibrations to equivalent uranium concentrations. A new field source (Ra-20S-82) was fabricated to replace the old source (Ra-20S-204), whose activity led to variable field calibration results previously caused by a nonfixed geometry. A cross-calibration study was performed to compare the operation of the new source relative to the old source. A digitally based collection/recording system was recently acquired so that many of the procedures were performed with the old analog system and the new digital system to compare the performance of the digital system.

5. Arthur, R. J. 1992. Yearly Calibration of Pacific Northwest Laboratory's Gross Gamma-Ray Borehole Geophysical Logging System, 1992. PNL-8220, Battelle Pacific Northwest Laboratories, Richland, Washington. ABSTRACT: This report describes the 1992 yearly calibration of a gross gamma-ray geophysical pulse-logging system owned by the U.S. Department of Energy (DOE) and operated by Pacific Northwest Laboratory. The calibration was conducted to permit the continued use of this system for geologic and hydrologic studies associated with remedial investigation at the Hanford Site. The calibration is limited to the probe identified as CG27A-97. Primary calibrations to equivalent-uranium units were conducted in DOE borehole model standards that reside on the Hanford Site. The calibrations were performed in borehole models SBL/SBH and SBA/SBB, which contain low equivalent-uranium concentrations. A previous correlation for relating observed count rate in before- and after-logging field calibrations to equivalent-uranium concentrations was confirmed for field source Ra-20S-82. A computer-based digital collection/ recording system was used simultaneously on many of the procedures with the original analog system so that the performance of the two collection systems could be correlated and compared at some future date.

6. Arthur, R. J. 1993. 1993 Yearly Calibration of Pacific Northwest Laboratory's Gross GammaRay Borehole Geophysical Logging System. PNL-8895, Battelle Pacific Northwest Laboratories, Richland, Washington. ABSTRACT: This report describes the 1993 yearly calibration of a gross gamma-ray geophysical pulse-logging system owned by the U.S. Department of Energy (DOE) and operated by Pacific Northwest Laboratory. The calibration was conducted to permit the continued use of this system for geologic and hydrologic studies associated with remedial investigation at the Hanford Site. The calibration is limited to the probe identified as CG27A-97 and applies to a new probe detector crystal that replaced the former detector in January 1993, after a crack was discovered. Primary calibrations to equivalent-uranium units were conducted in DOE borehole model standards that reside on the Hanford Site. The calibrations were performed in 
borehole models SBL/SBH and SBA/SBB, which both contain sections with relatively low equivalent-uranium concentrations. Model SBH/SBL has now been countersunk at the site so that data were taken by suspending the probe downhole. Model SBA/SBB is still lying horizontally above ground at the site, so data were logged as in previous calibrations at the Hanford Site by pushing the probe into the hole and drawing it out with the logging equipment. A previous correlation for relating observed count rate in before- and after-logging field calibrations to equivalent-uranium concentrations was confirmed for field source Ra-20S-82. A computer-based digital collection/recording system was used simultaneously on many of the procedures with the original analog system so that the performance of the two collection systems could be correlated and compared. The digital system was calibrated this year to confirm its linearity and to establish correlation with the analog data (i.e., chart-recorder output).

7. Battelle Pacific Northwest Laboratories. 1987. Groundwater Monitoring Compliance Projects for Hanford Site Facilities: Volume 3, Appendices C-G, Progress Report for the Period October 1 to December 31, 1986. PNL-6465, Vol. 3, Appendices C through G, Battelle Pacific Northwest Laboratories, Richland, Washington. ABSTRACT: This volume contains Appendices C through G. Appendix C contains the geophysical logs for 12 new wells in the 100-H Area. Appendix D contains the Statement of Work, which details the efforts needed to finish construction of the deep monitoring and observation wells at the Nonradioactive Dangerous Waste Landfill. Appendix E contains drilling logs, geologists' logs, and construction diagrams for the deep monitoring and observation wells at the Nonradioactive Dangerous Waste Landfill. Appendix F contains information on the hydrogeologic testing plan for new wells at the Nonradioactive Dangerous Waste Landfill. Included are the text of a presentation concerning this plan and an account of the discussion that followed. Appendix G contains raw analytical data from a set of samples collected in October 1986 from wells SM-1 through SM-5 at the Nonradioactive Dangerous Waste Landfill.

8. Battelle Pacific Northwest Laboratories. 1987. Groundwater Monitoring Compliance Projects for Hanford Site Facilities. PNL-6467 Vol. 3, Appendix C-I, Battelle Pacific Northwest Laboratories, Richland, Washington. ABSTRACT: Appendix C contains the raw analytical data collected from the groundwater monitoring networks for the 183-H Solar Evaporation Basins for December 1986 through February 1987. Appendix D contains information on particle size analysis, calcium carbonate determinations, soil moisture analysis, and low-permeability unit analysis for samples from monitoring wells in the area of the NRDW Landfill. Appendix E contains lithologic logs and well construction summaries for wells in the area of the NRDW Landfill. Appendix F contains geophysical logs for the wells in the area of the NRDW Landfill. The logs, gamma, neutron, and density are all plotted on graphs, one graph for each well. Appendix G provides aquifer test data, including pumping and observation well completions and locations, pumping rates, drawdown and recovery water-level data, and presentation and discussion of analyses. Appendix H provides water-level data collected at the Nonradioactive Dangerous Waste Landfill intended to provide information on the hydraulic gradients (horizontal and vertical) beneath the site. Appendix I contains measurements and samples taken from the pumping well discharge during aquifer tests and routine quarterly samples taken during the first two quarters of monitoring. 
9. Battelle Pacific Northwest Laboratories. 1988. Groundwater Monitoring Compliance Projects for Hanford Site Facilities: Progress Report for the Period April 1 to June 30, 1988: Volume 2, Appendices. PNL-6675 Vol. 2, Battelle Pacific Northwest Laboratories, Richland, Washington. ABSTRACT: This is Volume 2 of a two-volume set of documents that describes the progress of 10 Hanford Site groundwater monitoring projects for the period April 1 to June 30, 1988. This volume discusses as-built diagrams, drilling logs, and geophysical logs for wells drilled during this period in the 100-N Area (Appendix A) and near the 216-A-36B Crib (Appendix B). Volume 1 discusses the 10 projects.

10. Blair, S. C., L. S. Law, and J. W. Lindberg. 1981. A Catalog of Borehole Geophysics on the Hanford Site, 1958 to 1980. PNL-3504, Pacific Northwest Laboratory, Richland, Washington. ABSTRACT: This report catalogs geophysical borehole data acquired between January 1, 1958, and April 8, 1980, for nearly 800 wells on and around the Hanford Site. These data have been placed in a format that can be easily adapted to a computerized data management system. Maps are provided for quick identification of specific wells. Each well is classified according to the amount of geophysical logging data available. A cross reference between common well name and the Hanford grid name is given to eliminate confusion among various sources of data.

11. Brodeur, J. R. 1988. “Fiscal Year 1987 Inactive Crib Monitoring Report." Westinghouse Hanford Company Internal Memo 80230-88-004 to V. W. Hall. ABSTRACT: The results of gross gamma logging of about 140 wells associated with 39 inactive crib sites are presented in tabular form.

12. Brodeur, J. R., and C. J. Koizumi. 1989. Base Calibration of Pacific Northwest Laboratory's Gross Gamma Borehole Geophysical Logging System. WHC-EP-0246, Westinghouse Hanford Company, Richland, Washington. ABSTRACT: This report describes a base calibration of Pacific Northwest Laboratory's gross gamma geophysical logging system. The calibration was conducted to permit the use of this system for geologic and hydrologic studies associated with remedial investigation at the Hanford Site and to determine the suitability of the instrumentation for monitoring in areas with radiological contamination. A basic description of the system instrumentation is provided as well as a calibration check of this instrumentation, which measured linearity, dead-time, and instrument drift. A primary calibration to equivalent uranium units was conducted in Spokane, Washington, at a U.S. Department of Energy field calibration facility. This primary calibration was only partially successful because the system could not measure the activity in a calibration model zone containing a high uranium concentration. The primary calibration was, therefore, limited to system response in a zone with low uranium concentration. It is, therefore, impossible to determine the linearity of the conversion to equivalent uranium units with this system.

13. Brodeur, J. R. 1993. Assessment of Unsaturated Zone Radionuclide Contamination Around Single-Shell Tanks 241-C-105 and 241-C-106. WHC-SD-EN-TI-185, Rev. 0, Westinghouse Hanford Company, Richland, Washington. ABSTRACT: Spectral gamma-ray logs from 15 drywells around single-shell tanks 241-C-105 and -106 are presented. 
14. Brodeur, J. R., C. J. Koizumi, J. P. Meisner, R. K. Price, and J. W. Fassett. 1993. Application of Spectral Gamma-Ray Borehole Geophysics for Characterization of Radionuclide Contamination at Hanford. WHC-SA-2055, Westinghouse Hanford Company, Richland, Washington. ABSTRACT: An innovative, passive, gamma-ray borehole logging program was developed at Hanford for the purpose of identifying and quantifying the gamma-ray-emitting radioisotopes in the subsurface. Recent nuclear counting equipment was configured into a borehole logging system and calibration methods were devised for the in situ assay of man-made radionuclides. Operation procedures and data analysis methods were developed, creating an efficient production logging system. The logging program has proven to be effective when used in a screening mode for characterizing effluent release sites at Hanford. It located the primary gamma-emitting contaminants and identified which of the sites had contributed to the groundwater contamination. At one of the single-shell tank farms, the system was used to quantify the unsaturated zone contamination and trace the contamination back to the tank leak sources. It also provided a baseline of the radioelement concentrations for future data comparisons and to permit identification of a dynamic radionuclide transport system. In this manner, the logging program can help to identify future tank leaks. When combined with an inexpensive drilling method, the logging program can produce in situ radioelement concentration data at a fraction of the cost of conventional drilling and sampling, without personnel exposure to the contamination or generation of waste, and it can be done in a matter of days instead of months. Future developments will refine the quality assurance program so that the system will produce laboratory-quality in situ assays. Future applications will require a high degree of quality assurance.

15. Brodeur, J. R., and C. D. Wittreich. 1993. Assessment of Unsaturated Zone Radionuclide Contamination in the 200 Areas of the Hanford Site, Washington. WHC-SA-1907, Westinghouse Hanford Company, Richland, Washington. ABSTRACT: The 200 East and 200 West Areas at the U.S. Department of Energy's Hanford Site in southeastern Washington, contain chemical and nuclear fuel processing facilities that disposed of large volumes of chemical and radionuclide effluents to the ground via various structures such as ponds, cribs, and ditches. A geophysical logging investigation was implemented in 1992 to assess the nature and extent of contamination beneath select liquid disposal sites in the 200 Areas. The borehole geophysical logging was accomplished with a recently developed spectral gamma-ray logging system called the Radionuclide Logging System (RLS). This system has a high-resolution, intrinsic germanium detector mounted in a downhole probe and is calibrated and operated specifically for use in a borehole environment. It provides a means to develop in situ, gamma-emitting radioelement concentration profiles. Approximately 50 boreholes were logged in this study. The RLS log data provided information about the migration and deposition patterns of specific radionuclides in the unsaturated zone and their impacts on the groundwater. Approximately 10 radionuclide species were detected and quantified. Results of the field investigation are being used to refine site-specific conceptual models, support Hanford Site remediation decisions, and focus future characterization activities.

16. Brodeur, J. R., R. K. Price, R. D. Wilson, and C. J. Koizumi. 1993. Results of Spectral Gamma-Ray Logging of Select Boreholes for the Aggregate Area Management Study. WHC-SD-EN-TI-021, Rev. 0, Westinghouse Hanford Company, Richland, Washington. 
ABSTRACT: Spectral gamma-ray borehole geophysics was completed in boreholes at select liquid disposal sites within the 200 Areas. This was a limited field investigation conducted as part of the 200 Aggregate Area Management Study for the purpose of identifying the principal sources of groundwater contamination and providing some initial site characterization data. Gamma-rayemitting radionuclide concentration plots were prepared for 47 boreholes at 38 waste sites. These data were assessed to identify general radionuclide migration patterns. The data will contribute to the development of conceptual models for the liquid disposal sites and focus future ER characterization activities.

17. Brodzinski, R. L. 1981a. In Situ Subterranean Determination of Actinides by High-Resolution Gamma-Ray Spectrometry. PNL-SA-8992, Pacific Northwest Laboratory, Richland, Washington. ABSTRACT: A system utilizing high-resolution germanium diode gamma-ray spectroscopy for the simple, safe, and economical in situ determination of actinides is described. Six isotopes, ${ }^{235} \mathrm{U},{ }^{238} \mathrm{U},{ }^{237} \mathrm{~Np},{ }^{239} \mathrm{Pu},{ }^{241} \mathrm{Pu}$, and ${ }^{241} \mathrm{Am}$, can be simultaneously measured at the $10^{-1} \mathrm{nCi}$ g level in less than 7 minutes. Collimators provide for measurement of horizontal strata as thin as $1 \mathrm{~cm}$ or solid angles as small as 0.1 steradians. Information obtainable with the system is discussed and compared to that obtainable with neutron activation/detection systems.

18. Brodzinski, R. L. 1981b. Well-Logging Instrumentation. PNL-SA-10023, Pacific Northwest Laboratory, Richland, Washington. ABSTRACT: Research investigations on techniques for in situ determination of ${ }^{90} \mathrm{Sr}$, tritium, and transuranic isotopes are described. Results of neutron activation analysis experiments on ${ }^{90} \mathrm{Sr}$ and passive neutron detection experiments on transuranics are given.

19. Brodzinski, R. L. 1983a. State-of-the-Art In-Situ Determination of Transuranics. PNL-SA10184S, Pacific Northwest Laboratory, Richland, Washington. ABSTRACT: Two techniques for in situ analysis of transuranics are described. The first provides quantitative information in actinide-only environments by analyzing high energy gamma-rays with a high-resolution germanium diode spectrometer. This technique is simple, safe, fast, specific, economical, and can interrogate representative sample volumes for the simultaneous determination of six actinide isotopes. The disadvantage lies in its inability to operate in high fission product environments. For high fission product concentrations, a method is described using exposure metal plates, usually copper, and subsequent analysis of the transuranic-generated neutron-induced activation products in the plate specifically developed for use in high gamma-ray environments. The technique is not isotopespecific and requires a relatively long turn-around time, but has many desirable attributes not found in other techniques. One application of this technique where the plutonium concentration was measured as a function of depth in a high-level waste tank containing salt cake is presented in a table.

20. Brodzinski, R. L. 1982b. Contamination Levels and for Monitoring the Effectiveness of Decontamination and Decommissioning Activities. PNL-4744, Pacific Northwest Laboratory, Richland, Washington. ABSTRACT: Two completely portable high-resolution germanium diode spectrometer systems are described. These detectors are capable of measuring transuranics, activation products, and fission products, including ${ }^{90} \mathrm{Sr}$, at sensitivities below the uncontrolled 
release criteria. The detectors measure x-rays, gamma-rays, or bremsstrahlung radiation as required and have been calibrated for a variety of decontamination and decommissioning scenarios. A description of a new technology for the in situ determination of ${ }^{90} \mathrm{Sr}$ is given.

21. Brodzinski, R. L., and H. L. Nielson. 1980. A Well Logging Technique for the In Situ Determination of ${ }^{90} \mathrm{Sr}$. In Nuclear Instruments and Methods, Vol. 173, p. 299-301. NorthHolland Publishing Company. ABSTRACT: A technique for determining the ${ }^{90} \mathrm{Sr}$ concentration in soils and sediments by measurement of bremsstrahlung radiation in the energy range $60-230$ $\mathrm{keV}$ with a large intrinsic germanium diode capable of logging wells as small as 3 " in diameter is described. Corrections are made for changing background levels as a function of depth and for interferences from any other radionuclides present. $\mathrm{A}^{90} \mathrm{Sr}$ concentration as low as $9 \mathrm{nCi} \mathrm{cm}^{-3}$ can be measured in only $10 \mathrm{~s}$ in typical fission product gamma-ray activities $100 \mathrm{nCi} \mathrm{cm}^{-3}$. If no interfering radionuclides are present, ${ }^{90} \mathrm{Sr}$ concentrations of $<10 \mathrm{pCi} \mathrm{cm}^{-3}$ can be determined. The relationship between detector size and sensitivity is discussed. Detection limits are given for different counting times as a function of interfering activity levels.

22. Brodzinski, R. L., and N. A. Wogman. 1976. Californium-252 In Situ Activation and Photon Detection Techniques for Uranium Ore Deposit Evaluation. CONF-760316-4, Pacific Northwest Laboratory, Richland, Washington. ABSTRACT: Four different techniques are evaluated for borehole analysis of uranium and thorium ores. Methods involving 1) detection of fission product photons following ${ }^{252} \mathrm{Cf}$ activation, 2) detection of low-energy uranium and thorium gamma-rays, 3) direct measurement of the $1001-\mathrm{keV}$ photon from ${ }^{243} \mathrm{~Pa}$, a progeny of ${ }^{238} \mathrm{U}$, and 4) isotopic excitation $\mathrm{x}$-ray fluorescence spectroscopy are evaluated. The first two techniques are found too unsuitable for most low-grade ores. The third is found to be suitable for the in situ analysis of uranium ores only, and the fourth method is shown to be a superior, cost-effective method for both uranium and thorium ore analysis.

23. Brown, D. J., R. C. Routson, W. H. Price, and K. R. Fecht. 1979. Status of Liquid Waste Leaked from the 241-T-106 Tank. RHO-ST-1, Rockwell Hanford Operations, Richland, Washington. ABSTRACT: On June 8, 1973, the 241-T-106 tank, located on the U.S. Department of Energy's Hanford Site, was confirmed as leaking. Approximately 4.35 x $10^{5}$ liters (115,000 gallons) of liquid containing 40,000 curies of cesium-137, 14,000 curies of strontium-90, 6 curies of plutonium and americium, and 297,000 curies of various fission products (with halflives less than 5 years) were released to the glaciofluvial sediments surrounding the tank. Subsequent to the leak, studies were conducted to monitor and assess the potential for migration of radioactivity to points of potential uptake. These studies included: the drilling of monitoring wells to provide access for in situ instrumentation and to collect sediment samples for geologic and radionuclide analysis; in situ total gamma logging of wells to monitor changes in leak plume; in situ gamma energy analysis measurements to determine species and concentrations of radionuclides in the leak plume; and computer modeling to predict future changes in the plume.

24. Caggiano, J. A. 1992. Borehole Completion Data Package for the CY 1990 Single-Shell Tank Drilling Project. WHC-SD-EN-DP-041, Westinghouse Hanford Company, Richland, Washington. ABSTRACT: This report contains gross gamma-ray logs for ten new boreholes. 
25. Caggiano, J. A. 1993. Borehole Completion Data Package for CY 1991 and CY 1992 RCRA Wells at Single-Shell Tanks. WHC-SD-EN-DP-042, Westinghouse Hanford Company, Richland, Washington. ABSTRACT: This report contains gross gamma-ray logs for the ten new wells.

26. Chamness, M. A., S. S. Teel, D. L. McAlister, A. W. Pearson, K.R.O. Barton, R. W. Fruland, and R. E. Lewis. 1991. U-Plant Aggregate Area Management Study Geologic Data Package. WHC-SD-EN-DP-019, Westinghouse Hanford Company, Richland, Washington.

ABSTRACT: This data package includes all of the geologic and geophysical information requested by the Westinghouse Hanford Company for the U-Plant Aggregate Area. This information includes a listing of all of the boreholes within this aggregate area as defined by the Westinghouse Hanford Company and amended by the Pacific Northwest Laboratory. Geophysical data (Table 1) includes the type of log, date, and interval logged for each of the above wells, as well as the location where these data are stored. Table 2 includes the types of geologic data available for each of these wells (i.e., driller's log, geologist's log) and any other related data (sieve, $\mathrm{CaCO}_{3}$ content, moisture content, and chemical analyses). Table 3 provides a summary of the well construction information for each well including drill depth, completion depth, screened interval, and coordinates.

27. Chamness, M. A., R. E. Lewis, S. S. Teel, and A. W. Pearson. 1991. T Plant Geologic and Geophysics Data Package for the 200 Aggregate Area Management Study. WHC-SD-EN-DP022, Westinghouse Hanford Company, Richland, Washington. ABSTRACT: This data package includes all of the geologic and geophysical information requested by the Westinghouse Hanford Company for the T-Plant Aggregate Area. This information includes a listing of all of the boreholes within this aggregate area as defined by the Westinghouse Hanford Company and amended by the Pacific Northwest Laboratory. Geophysical data (Table 1) includes the type of log, date, and interval logged for each of the above wells, as well as the location where these data are stored. Table 2 includes the types of geologic data available for each of these wells (i.e., driller's $\log$, geologist's log) and any other related data (sieve, $\mathrm{CaCO}_{3}$ content, moisture content, and chemical analyses). Table 3 provides a summary of the well construction information for each well including drill depth, completion depth, screened interval, and coordinates.

28. Chamness, M. A., S. S. Teel, D. L. McAlister, A. W. Pearson, K.R.O. Barton, R. W. Fruland, and R. E. Lewis. 1992. Z-Plant Aggregate Area Management Study Geologic Data Package. WHC-SD-EN-DP-020, Rev. 1, Westinghouse Hanford Company, Richland, Washington. ABSTRACT: This data package includes all of the geologic and geophysical information requested by the Westinghouse Hanford Company for the Z-Plant Aggregate Area. This information includes a listing of all of the boreholes within this aggregate area as defined by the Westinghouse Hanford Company and amended by the Pacific Northwest Laboratory. Geophysical data (Table 1) includes the type of log, date, and interval logged for each of the above wells, as well as the location where these data are stored. Table 2 includes the types of geologic data available for each of these wells (i.e., driller's log, geologist's log) and any other related data (sieve, $\mathrm{CaCO}_{3}$ content, moisture content, and chemical analyses). Table 3 provides a summary of the well construction information for each well including drill depth, completion depth, screened interval, and coordinates. 
29. Chamness, M. A., D. L. McAlister, J. P. McDonald, A. W. Pearson, and R. E. Lewis. 1992. 200 North Aggregate Area Management Study Geologic Data Package. Westinghouse Hanford Company, Richland, Washington. ABSTRACT: This data package includes all of the geologic and geophysical information requested by the Westinghouse Hanford Company for the 200 North aggregate area. This information includes a listing of all of the boreholes within this aggregate area as defined by the Westinghouse Hanford Company and amended by the Pacific Northwest Laboratory. Geophysical data (Table 1) includes the type of log, date, and interval logged for each of the above wells, as well as the location where these data are stored. Table 2 includes the types of geologic data available for each of these wells (i.e., driller's log, geologist's log) and any other related data (sieve, $\mathrm{CaCO}_{3}$ content, moisture content, and chemical analyses). Table 3 provides a summary of the well construction information for each well including drill depth, completion depth, screened interval, and coordinates.

30. Chamness, M. A., S. S. Teel, D. L. McAlister, A. W. Pearson, and R. E. Lewis. 1992. S-Plant Aggregate Area Management Study Geologic Data Package. WHC-SD-EN-DP-021, Westinghouse Hanford Company, Richland, Washington. ABSTRACT: This data package includes all of the geologic and geophysical information requested by the Westinghouse Hanford Company for the S-Plant Aggregate Area. This information includes a listing of all of the boreholes within this aggregate area as defined by the Westinghouse Hanford Company and amended by the Pacific Northwest Laboratory. Geophysical data (Table 1) includes the type of log, date, and interval logged for each of the above wells, as well as the location where these data are stored. Table 2 includes the types of geologic data available for each of these wells (i.e., driller's log, geologist's $\log$ ) and any other related data (sieve, $\mathrm{CaCO}_{3}$ content, moisture content, and chemical analyses). Table 3 provides a summary of the well construction information for each well including drill depth, completion depth, screened interval, and coordinates.

31. Chamness, M. A., R. E. Lewis, S. S. Teel, R. J. Brockman, D. C. Lanigan, and A. W. Pearson. 1992. PUREX Aggregate Area Management Study Geologic Data Package. WHC-SD-EN-DP025, Westinghouse Hanford Company, Richland, Washington. ABSTRACT: This data package includes all of the geologic and geophysical information requested by the Westinghouse Hanford Company for the PUREX Aggregate Area. This information includes a listing of all of the boreholes within this aggregate area as defined by the Westinghouse Hanford Company and amended by the Pacific Northwest Laboratory. Geophysical data (Table 1) includes the type of log, date, and interval logged for each of the above wells, as well as the location where these data are stored. Table 2 includes the types of geologic data available for each of these wells (i.e., driller's $\log$, geologist's $\log$ ) and any other related data (sieve, $\mathrm{CaCO}_{3}$ content, moisture content, and chemical analyses). Table 3 provides a summary of the well construction information for each well including drill depth, completion depth, screened interval, and coordinates.

32. Chamness, M. A., S. M. Goodwin, S. S. Teel, and R. E. Lewis. 1992. Semi-Works (C-Plant) Aggregate Area Management Study Geologic Data Package. WHC-SD-EN-DP-027, Westinghouse Hanford Company, Richland, Washington. ABSTRACT: This data package includes all of the geologic and geophysical information requested by the Westinghouse Hanford Company for the Semi-Works (C-Plant) Aggregate Area. This information includes a listing of all of the 
boreholes within this aggregate area as defined by the Westinghouse Hanford Company and amended by the Pacific Northwest Laboratory. Geophysical data (Table 1) includes the type of log, date, and interval logged for each of the above wells, as well as the location where these data are stored. Table 2 includes the types of geologic data available for each of these wells (i.e., driller's $\log$, geologist's log) and any other related data (sieve, $\mathrm{CaCO}_{3}$ content, moisture content, and chemical analyses). Table 3 provides a summary of the well construction information for each well including drill depth, completion depth, screened interval, and coordinates.

33. Chamness, M. A. 1986. "Fiscal Year 1986 Scintillation Logging Status." Rockwell Hanford Operations Internal Memo 65633-86-107 to V. W. Hall. ABSTRACT: The results of gross gamma logging of $\sim 122$ wells are presented in tabular form.

34. Delaney, C. D. 1992. Borehole Completion Data Package for the CY 1991 216-B-3 Pond Drilling Project. WHC-SD-EN-DP-046, Westinghouse Hanford Company, Richland, Washington. ABSTRACT: This report contains gross gamma-ray logs for seven new wells.

35. Dorian, J. J. 1995. “Transmittal of Sodium Iodide Acquired Data Processed for Potassium, Uranium, and Thorium for the Environmental Restoration Disposal Facility Project." Westinghouse Hanford Company Internal Memo, 9550298 to K. R. Fecht.

36. Dorian, J. J. 1998. "In Situ Gamma-Ray Survey Results of Selected Boreholes Associated with B-BX-BY Tank Farm Reassessment.” Waste Management Federal Services, Inc., Northwest Operations Letter, WMNW-9759114 to R. M. Smith. ABSTRACT: Pacific Northwest National Laboratory requested high-purity germanium spectral gamma-ray logs of the radionuclide distribution and concentration along the entire borehole length for 16 selected groundwater monitoring wells associated with the B-BX-BY tank farm reassessment. The logging results are presented.

37. Drnevich, V. P., R. Salgado, A. Ashmawy, W. P. Grant, and P. Vallenas. 1995. Interpretation of Large-Strain Geophysical Crosshole Tests. WHC-AS-2959, Westinghouse Hanford Company, Richland, Washington. ABSTRACT: A seismic crosshole test has been developed where large dynamic forces are applied in a borehole. This paper provides an improved, systematic interpretation scheme for the data from these large-strain geophysical crosshole tests. Use is made of both the measured velocities at each sensor and the travel times. The measured velocity at each sensor location shows a good measure of the soil particle velocity at that location. Travel times to specific features on the velocity time history, such as first crossover, are used to generate travel time curves for the waves, which are nonlinear. At some distance, the amplitudes reduce to where the stress-strain behavior is essentially linear and independent of strain amplitude. This fact is used together with the measurements at the three sensor locations in a rational approach for fitting curves of shear wave velocity versus distance from the source hole that allow the determination of the shear wave velocity and the shear strain amplitude at each of the sensor locations as well as the shear wave velocity associated with small-strain (linear) behavior. 
38. Elbring, G. J., and S. M. Narbutovskih. 1994. Integrated Test Plan for Crosswell Compressional and Shear Wave Seismic Tomography for Site Characterization at the VOC Arid Site. WHC-SD-EN-TI-039, Westinghouse Hanford Company, Richland, Washington. ABSTRACT: This integrated test plan describes the demonstration of the crosswell acoustic tomography technique as part of the Volatile Organic Compounds-Arid Integrated Demonstration (VOC-Arid ID). The purpose of this demonstration is to image the subsurface seismic velocity structure and to relate the resulting velocity model to lithology and saturation. In fiscal year (FY) 1994 an initial fielding will test three different downhole sources at two different sites at the U.S. Department of Energy Hanford Site to identify which sources will provide the energy required to propagate between existing steel-cased wells at these two sites. Once this has been established, a second fielding will perform a full compressional and shear wave tomographic survey at the most favorable site. Data reduction, analysis, and interpretation of this full data set will be completed by the end of this fiscal year. The specific need is detailed subsurface characterization with minimum intrusion. This technique also has applications for long-term vadose zone monitoring for both Resource Conservation and Recovery Act (RCRA) waste storage facilities and for remediation monitoring. Images produced are continuous between boreholes. This is a significant improvement over the single point data derived solely from core information. Saturation changes, either naturally occurring (e.g., perched water tables) or remediation-induced (e.g., water table mounding from injection wells or during inwell air sparging), could be imaged. These crosswell data allow optimal borehole placement for groundwater remediation, associated monitoring wells, and possibly evaluation of the effective influence of a particular remediation technique.

39. Elbring, G. J. 1995. Comparison of Lower-Frequency $(<1000 \mathrm{~Hz})$ Downhole Seismic Sources for Use at Environmental Sites. SAND-95-0344C, Sandia National Laboratories, Albuquerque, New Mexico. ABSTRACT: In conjunction with crosswell seismic surveying being done at the Hanford Site in south-central Washington, four different downhole seismic sources have been tested between the same set of boreholes. The four sources evaluated were the Bolt airgun, the OYO-Conoco orbital vibrator, and two Sandia-developed vertical vibrators, one pneumatically driven, and the other based on a magnetostrictive actuator. The sources generate seismic energy in the lower frequency range of less than $1000 \mathrm{~Hz}$ and have different frequency characteristics, radiation patterns, energy levels, and operational considerations. Collection of identical data sets with all four sources allows the direct comparison of these characteristics and an evaluation of the suitability of each source for a given site and target.

40. Engelmann, R. E., R. E. Lewis, and D. C. Stromswold. 1995. Calibration Models for Density Borehole Logging - Construction Report. PNL-10800, Battelle Pacific Northwest Laboratories, Richland, Washington. ABSTRACT: Two machined blocks of magnesium and aluminum alloys form the basis for Hanford's density models. The blocks provide known densities of $1.780 \pm$ $0.002 \mathrm{~g} / \mathrm{cu} \mathrm{cm}$ and $2.804 \pm 0.002 \mathrm{~g} / \mathrm{cu} \mathrm{cm}$ for calibrating borehole logging tools that measure density based on gamma-ray scattering from a source in the tool. Each block is $\sim 33 \times 58 \times 91 \mathrm{~cm}$ (13 $\times 23 \times 36$ in.) with cylindrical grooves cut into the sides of the blocks to hold steel casings of inner diameter $15 \mathrm{~cm}$ (6 in.) and $20 \mathrm{~cm}$ (8 in.). Spacers that can be inserted between the blocks and casings can create air gaps of thickness $0.64,1.3,1.9$, and $2.5 \mathrm{~cm}(0.25,0.5,0.75$, and $1.0 \mathrm{in}$.), simulating air gaps that can occur in actual wells from hole enlargements behind the casing. 
41. Engelman, R. E., R. E. Lewis, and D. C. Stromswold. 1995. Calibration Models for Measuring Moisture in Unsaturated Formations by Neutron Logging. PNL-10801, Battelle Pacific Northwest Laboratories, Richland, Washington. ABSTRACT: Calibration models containing known amounts of hydrogen have been constructed to simulate unsaturated earth formations for calibrating neutron well-logging tools. The models are made of dry mixtures of hydrated alumina $(\mathrm{Al}(\mathrm{OH}) 3)$ with either silica sand $(\mathrm{SiO} 2)$ or aluminum oxide (A12O3). Hydrogen in the hydrated alumina replaces the hydrogen in water for neutron scattering, making it possible to simulate partially saturated formations. The equivalent water contents for the models are 5\%,12\%, 20\%, and 40\% by volume in seven tanks that have a diameter of $1.5 \mathrm{~m}$ and a height of $1.8 \mathrm{~m}$. Steel casings of inside diameter $15.4 \mathrm{~cm}$ (for three models) and diameter $20.3 \mathrm{~cm}$ (for four models) allow logging tool access to simulate logging through cased boreholes.

42. Fassett, J. W. 1993. "Spectral Gamma-Ray Log Report of Radionuclide Surveys Acquired for 216-U-17 Crib." Westinghouse Hanford Company Internal Memo, 81234-93-022, to S. P. Reidel. ABSTRACT: Logging with the high-resolution, high-purity germanium passive spectral gamma-ray system was completed for six boreholes along the 216-U-17 crib.

43. Fassett, J. W. 1995. “299-E33-05 Contamination Movement.” Westinghouse Hanford Company Internal Memo, 9550759 to M. A. Buckmaster.

44. Fecht, K. R., G. V. Last, and K. R. Price. 1977. Evaluation of Scintillation Probe Profiles from 200 Area Crib Monitoring Wells, Volumes 1 through 3. ARH-ST-156, Atlantic Richfield Hanford Company, Richland, Washington. ABSTRACT: Approximately 300 monitoring wells adjacent to over 100 cribs in the 200 Areas were logged with scintillation probes in 1976. Scintillation probe profiles were developed from these logs taken between 1954 and 1973. The profiles were compiled to measure qualitatively the distribution, redistribution, and decay of radioactive contaminants disposed to the ground beneath crib facilities.

45. Fecht, K. R. 1992. "Spectral Gamma-Ray Log Report for 300-FF-5 Borehole Surveys." Westinghouse Hanford Company Internal Memo, 81230-92-034 to L. C. Holstrom.

46. Fecht, K. R. 1992. "Spectral Gamma-Ray Log Report for 299-W18-98." Westinghouse Hanford Company Internal Memo, 81230-92-028 to J. M. Jimenez.

47. Fecht, K. R. 1992. "Spectral Gamma-Ray Log Report for HWVP." Westinghouse Hanford Company Internal Memo, 81230-92-024 to M. A. Wasemiller.

48. Fecht, K. R. 1992. "Spectral Gamma-Ray Survey Report for Borehole 699-41-35." Westinghouse Hanford Company Internal Memo, 81230-92-045 to J. D. Davis. ABSTRACT: Spectral gamma-ray log of well 699-41-35 at the 200 Area Treated Effluent Disposal Basin is presented. 
49. Fecht, K. R. 1992. "Spectral Gamma-Ray Log Report for 100 Area Borehole Surveys." Westinghouse Hanford Company Internal Memo, 81230-92-038 to A. D. Krug. ABSTRACT: Spectral gamma-ray logs from 40 boreholes in the 100-BC-1, 100-BC-5, 100-DR-1, 100-FR-3, 100-HR-1, 100-HR-3, and the 100-NR-2 operable units are presented.

50. Fecht, K. R. 1992. "Spectral Gamma-Ray Survey Report for Soil Column Disposal Site." Westinghouse Hanford Company Internal Memo, 81230-92-048 to S. P. Reidel. ABSTRACT: Spectral gamma-ray logs of wells 699-48-77 and 699-48-77A are presented.

51. Fecht, K. R. 1993. "Spectral Gamma-Ray Log Report for Nine Additional 200-BP-1 Borehole Surveys.” Westinghouse Hanford Company Internal Memo, 81230-93-003 to M. A. Buckmaster.

52. Fecht, K. R. 1993. "Spectral Gamma-Ray Log Report of Radionuclide Surveys Acquired for 100-KR-1 Operable Unit.” Westinghouse Hanford Company Internal Memo, 81230-93-009 to N. M. Naiknimbalker. ABSTRACT: Spectral gamma-ray logs from wells 116-K-2, 116-KE-4A, and 116-KW-3A and a gross gamma log from well 116-K-1 are presented.

53. Fecht, K. R. 1993. "Spectral Gamma-Ray Log Report of Radionuclide Surveys Acquired for 100-FR-3 Operable Unit.” Westinghouse Hanford Company Internal Memo, 81230-93-011 to J. W. Roberts. ABSTRACT: Spectral gamma-ray logs from boreholes 199-F1-2, 199-F5-47, 199-F7-3, and 199-F8-3 are presented.

54. Fecht, K. R. 1993. "Spectral Gamma-Ray Log Report of Radionuclide Survey Acquired for 216-Z-20 Crib Restart.” Westinghouse Hanford Company Internal Memo, 81230-93-012 to V. G. Johnson. ABSTRACT: Spectral gamma-ray logs of boreholes 299-W18-17, -18, -19, -20, and -29 are presented.

55. Fruland, R. M., D. J. Bates, and R. E. Lundgren. 1989. Resource Conservation and Recovery Act Groundwater Monitoring Projects for Hanford Facilities: Progress Report for the Period July 1 to September 30, 1988: Volume 1, Text. PNL-6789, Vol. 1, Battelle Pacific Northwest Laboratories, Richland, Washington. ABSTRACT: This report describes the progress of 12 Hanford groundwater monitoring projects for the period July 1 to September 30, 1988. During this quarter, field activities at the 300 Area process trenches, the Nonradioactive Dangerous Waste Landfill, the 183-H Solar Evaporation Basins, the 1324-N/NA Surface Impoundment and Percolation Ponds, the 1301-N and 1325-N Liquid Waste Disposal Facilities, and the 216-A-36B Crib consisted of groundwater sampling and analyses, and water-level monitoring. The 200 Area LowLevel Burial Grounds section includes well development data, sediment analysis, and water-level measurements. Groundwater sampling was begun at this site, and results will be included in next quarter's report. Twelve new wells were installed during the quarter, two at the 216-A-29 Ditch, six at the 216-A-10 Crib, and four at the 216-B-3 Pond. Preliminary characterization data for these new wells are included in this report. Drillers' logs and other drilling and site characterization data will be provided in the next quarterly report. At the 2101-M Pond, construction was completed on 
four wells, and initial groundwater samples were taken. The drilling logs, geophysical logging data, and as-built diagrams are included in this report in Volume 2.

56. Fruland, R. M., D. J. Bates, and R. E. Lundgren. 1989. Resource Conservation and Recovery Act Groundwater Monitoring Projects for Hanford Facilities: Progress Report for the Period July 1 to September 30, 1988: Volume 2. Appendices. PNL-6789, Vol. 2, Battelle Pacific Northwest Laboratories, Richland, Washington. ABSTRACT: This is Volume 2 of a twovolume set of documents that describes the progress of 12 Hanford Site groundwater monitoring projects for the period July 1 to September 30, 1988. This volume provides those drilling logs and well inspection/completion reports inadvertently left out of last quarter's report for the 216-A-36B Crib (Appendix A) and as-built diagrams, drilling logs, and geophysical logs for wells drilled this quarter near the 2101-M Pond. Volume 1 discusses the 12 projects.

57. Fruland, R. M., D. J. Bates, and R. E. Lundgren. 1989. RCRA (Resource Conservation and Recovery Act of 1976) Groundwater Monitoring Projects for Hanford Facilities: Progress Report, October 1-December 31, 1988: Volume 1. Text. PNL-6844 Vol. 1, Battelle Pacific Northwest Laboratories, Richland, Washington. ABSTRACT: This report describes the progress of 13 Hanford groundwater monitoring projects for the period October 1 to December 31, 1988. There are 16 individual hazardous waste facilities covered by the 13 groundwater monitoring projects. The Grout Treatment Facility is included in this series of quarterly reports for the first time. The 13 projects discussed in this report were designed according to applicable interim-status groundwater monitoring requirements specified in the Resource Conservation and Recovery Act of 1976 (RCRA). During this quarter, field activities primarily consisted of sampling and analyses, and water-level monitoring. The 200 Areas Low-Level Burial Grounds section includes sediment analyses in addition to groundwater monitoring results. Twelve new wells were installed during the previous quarter: two at the 216-A-29 Ditch, six at the 216-A-10 Crib, and four at the 216-B-3 Pond. Preliminary characterization data for these new wells include drillers' logs and other drilling and site characterization data, and are provided in Volume 2 or on microfiche in the back of Volume 1.

58. Gardner, M. G. 1998. “In Situ Spectral Gamma-Ray Soil Analysis Results of Auger Drilled Boreholes for WESF Low Level Liquid Waste Underground Piping Trench.” Waste Management Federal Services, Inc., Northwest Operations Letter, WMNW-025-MGG.98 to W. R. Shannon. ABSTRACT: B\&W Hanford Company requested auger drilling and high-purity germanium (HPGe) spectral gamma-ray analysis of the radionuclide distribution and concentration of the sediments between tank vault TK-100 and the Waste Encapsulation Storage Facility Truck Port to a depth of $1.5 \mathrm{~m}(5 \mathrm{ft})$. The results of the survey are presented.

59. George, D. C., and R. D. Wilson. 1994. Demonstration of Prompt Fission Neutron Logging Tool Response to Fissile Elements. Unpublished manuscript, prepared by RUST Geotech, Inc. for U.S. Department of Energy, Grand Junction Projects Office, Albuquerque Operations Office, Albuquerque, New Mexico. ABSTRACT: From about 1975 to 1985 the U.S. Department of Energy Grand Junction Project Office managed the National Uranium Resource Evaluation program. This program included development of borehole logging technologies. One of these, 
known as Prompt Fission Neutron logging, was developed by Sandia National Laboratories. In 1978, during that development, a short test of the technology was done at Hanford to show the technology's capability to measure Pu in situ. At the termination of the National Uranium Resource Evaluation program, the Prompt Fission Neutron technology was transferred to the Grand Junction Project Office contractor where it was maintained in mothballs. The hardware was briefly revived and taken again to Hanford for another short test in 1984. It was again retrieved from mothballs as part of the DOE Technology Development Program in 1993 and taken to Hanford for a third test. Hanford was chosen for this third test because no suitable holes were available at the Idaho National Engineering Laboratory's Radioactive Waste Management Complex, as proposed. This report describes the system, its calibration, and its capabilities and performance. It compares $\mathrm{Pu}-239 \operatorname{logs}$ collected three times over a period of fifteen years in the same two boreholes.

60. Hartman, M. J. 1992. Borehole Completion Data Package for Wells N-71, N-72, N-73, and $\mathrm{N}$-74. WHC-SD-EN-DP-040, Westinghouse Hanford Company, Richland, Washington. ABSTRACT: This report contains gross gamma-ray logs for four new wells.

61. Hartman, M. J. 1992. Borehole Completion Data Package, 100-D Ponds Wells: CY 1992. WHC-SD-EN-DP-043, Westinghouse Hanford Company, Richland, Washington.

ABSTRACT: This report contains gross gamma-ray logs for four new wells.

62. Heistand, B. E., and E. F. Novak. 1984. Parameter Assignments for Spectral Gamma-Ray Borehole Calibration Models. GJBX-2(84). Bendix Field Engineering Corporation, Grand Junction, Colorado.

63. Horton, D. G. 1992. “Review of RLS Surveys in 100 Area Drilling Boreholes." Westinghouse Hanford Company Internal Memo, 81232-92-008 to A. D. Krug.

64. Horton, D. G. 1998. Monitoring Plan for Borehole Logging at 216-Z-1A Tile Field, 216-Z-9 Trench, and 216-Z-12 Crib. PNNL-11878, Pacific Northwest National Laboratory, Richland, Washington. ABSTRACT: This plan describes the fiscal year 1998 vadose monitoring of three inactive, liquid waste disposal facilities associated with the Plutonium Finishing Plan (Z-Plant): the 216-A-1A tile filed, the 216-Z-9 trench, and the 216-Z-12 crib. Monitoring will consist of spectral gamma-ray logging of twenty-one boreholes. This plan described the physical characteristics of the facilities, their operational histories, the subsurface geology, and known contamination distribution at each facility. The plan then describes the specific monitoring to be done including the boreholes to be logged; the methods of data acquisition, data reduction, and data evaluation; and the quality control, data management, and data reporting for this effort.

65. Horton, D. G. 1999. Monitoring Plan for Fiscal Year 1999 Borehole Logging at 200 East Area Specific Retention Facilities. PNNL-12222, Pacific Northwest National Laboratory, Richland, Washington. ABSTRACT: The Hanford Groundwater Monitoring Project's vadose zone monitoring effort for fiscal year 1999 involves monitoring 30 boreholes for moisture content and gamma-ray-emitting radionuclides. This monitoring plan describes the facilities and the vadose zone at the cribs and trenches to be monitored; the field activities to be accomplished; the 
constituents of interest and the monitoring methods, including calibration issues; and the quality assurance and quality control requirements governing the monitoring effort.

66. Horton, D. G., and R. R. Randall. 1998. Results of 1998 Spectral Gamma-Ray Monitoring of Boreholes at the 216-Z-1A Tile Field, 216-Z-9 Trench, and 216-Z-12 Crib. PNNL-11978, Pacific Northwest National Laboratory, Richland, Washington. ABSTRACT: In April 1998, 21 boreholes at the 216-Z-1A tile filed, 216-Z-9 trench, and 216-Z-12 crib were monitored by spectral gamma-ray logging because they were identified as containing some of the most significant sources of radioactive contamination in the Hanford Site vadose zone. The 1998 logging revealed that man-made isotopes (cesium-137, protactinium-233, plutonium-239, and americium-241) were present in the 216-Z-1A tile field and the 216-Z-12 crib. The data collected in 1998 were compared with past log data, suggesting that there have been some changes in radionuclide activity around two boreholes at the tile field and one borehole at the crib.

67. Horton, D. G., and R. R. Randall. 2000. Results of 1999 Spectral Gamma-Ray and Neutron Moisture Monitoring of Boreholes at Specific Retention Facilities in the 200 East Area, Hanford Site. PNNL-13077, Pacific Northwest National Laboratory, Richland, Washington.

ABSTRACT: Twenty-eight wells and boreholes in the 200 East Area, Hanford Site, Washington, were monitored in 1999. The monitored facilities were past-practice liquid waste disposal facilities and consisted of six cribs and nineteen specific retention cribs and trenches. Monitoring consisted of spectral gamma-ray and neutron moisture logging. All data are included in Appendix B.

68. Horton, D. G., S. P. Reidel, and G. V. Last. Monitoring Guidance for Vadose Zone Monitoring of Liquid Waste Disposal Facilities for the Hanford Groundwater Project. PNNL-11958, Rev. 2, Pacific Northwest National Laboratory, Richland, Washington. ABSTRACT: This document is prepared by Pacific Northwest National Laboratory in response to a U.S. Department of Energy request for a description of vadose zone monitoring of liquid waste disposal facilities that are not part of the Tank Waste Remediation System. This document includes the needs and objectives of vadose zone monitoring and provides a rationale and general framework for vadose zone monitoring of past-practice cribs, ditches, trenches, and other disposal facilities. The monitoring described herein will be modified as necessary so as to be incorporated into the Groundwater/ Vadose Zone Integration Project. The vadose zone monitoring addressed in this document is part of the Hanford Groundwater Monitoring Project. As such, the major objective of the monitoring is protection of groundwater. However, the Hanford Groundwater Monitoring Project's vadose zone monitoring complements the 200 Area soil remediation strategy developed by the Hanford Site's Environmental Restoration Contractor. Most 200 Areas past-practice, liquid waste, disposal facilities are slated for remediation, ranging from complete cleanup to in-place management. The monitoring discussed in this document will track vadose zone contamination until remedial actions can occur. Also, if in some instances vadose contamination is managed in place, periodic monitoring to assess the effectiveness of that remedial decision may be necessary. It is expected that the Hanford Groundwater Monitoring Project's vadose zone activities will undergo changes as the priorities and activities of the Hanford Site environmental restoration activities evolve. 
69. Isaacson, R. E., and K. A. Gasper. 1981. A Scientific Basis for Establishing Dry WellMonitoring Frequencies. RHO-ST-34, Rockwell Hanford Operations, Richland, Washington. ABSTRACT: A scientific basis has been developed for establishing the frequency of monitoring dry wells. Dry wells are used to detect radioactivity from a leaking underground high-level radioactive waste-storage tank. The frequency of monitoring a dry well is dependent on the response characteristics of the radiation-detection system periodically used to monitor dry wells for encroaching radioactivity.

70. Johnson, V. G. 1993. Groundwater Impact Assessment Report for the 16-Z-20 Crib, 200 West Area. WHC-EP-0674, Westinghouse Hanford Company, Richland, Washington. ABSTRACT: Neutron, total gamma, and natural potassium, uranium, and thorium logs are presented for boreholes 299-W18-17, -18, -19, -20, and -29. Spectral gamma logs are presented for boreholes 299-W18-159 and -171 .

71. Kasper, R. B. 1981. 216-Z-12 Crib Status Report. RHO-LD-166, Rockwell Hanford Operations, Richland, Washington. ABSTRACT: This report gives the results of six copper foil experiments and one neutron log at the 216-Z-12 crib.

72. Kasper, R. B. 1982. 216-Z-12 Transuranic Crib Characterization: Operational History and Distribution of Plutonium and Americium. RHO-ST-44, Rockwell Hanford Operations, Richland, Washington. ABSTRACT: This report presents one neutron flux log.

73. Kasza, G. L. 1992. Borehole Completion Data Package for 216-A-29 RCRA Facility Monitoring Wells 299-E25-42, 299-E25-43, 299-E26-12, and 299-E26-13. WHC-SD-EN-DP047, Westinghouse Hanford Company, Richland, Washington. ABSTRACT: This report contains gross gamma-ray logs for the four new wells.

74. Koegler, K. J. 1993. Integrated Test Plan for Demonstration of a Prompt Fission Neutron Logging Tool. WHC-SD-EN-TI-032, Westinghouse Hanford Company, Richland, Washington. ABSTRACT: This plan describes the demonstration of the geophysical logging tool as part of the Volatile Organic Compounds Arid Sites Integrated Demonstration (VOC-Arid ID) and the Buried Waste Integrated Demonstration. The purpose is to show that the tool can log cased boreholes at Hanford waste sites for the presence in the soil of fissionable isotopes.

75. Koizumi, C. J. 1990. "Spectral Gamma Ray Log From Borehole 299-W26-11." Westinghouse Hanford Company Internal Memo, 81232-91-032 to B. A. Williams.

76. Koizumi, C. J., J. R. Brodeur, W. H. Ulbricht, and R. K. Price. 1991. Calibration of the RLS HPGe Spectral Gamma Ray Logging System. WHC-EP-0464, Westinghouse Hanford Company, Richland, Washington. ABSTRACT: Gamma-ray spectral data have been recorded with the Radionuclide Logging System (RLS) high-purity germanium (HPGe) system at 1) the American Petroleum Institute (API) spectral gamma-ray calibration center in Houston, Texas; 2) the U.S. Department of Energy (DOE) spectral gamma-ray field calibration facility in Spokane, Washington; and 3) the DOE spectral gamma-ray primary calibration center in Grand Junction, Colorado. 
Analyses of the Grand Junction data yielded: calibration constants for the natural gamma-ray sources (potassium, uranium and thorium), energy-dependent borehole diameter corrections for the air-filled borehole, energy-dependent borehole casing corrections for steel casing over a range of thickness from 0 to $79 \mathrm{~cm}(5 / 16 \mathrm{in}$.), a casing index function that varies with casing thickness and provides a method for verifying that the correct casing correction is applied, and an energydependent inverse function that is the basis for assessment of subsurface concentrations of manmade gamma-ray emitters such as cesium-137 and cobalt-60.

77. Koizumi, C. J. 1991. “Borehole Logging Activity Report for Interim Response Action: Carbon-Tetrachloride Vapor Extraction." Westinghouse Hanford Company Internal Memo, 81232-91-021 to V. J. Rohay. ABSTRACT: Gross gamma and spectral gamma logs from boreholes 299-W18-87, -150, -164, and -171 are presented.

78. Koizumi, C. J., W. H. Ulbricht, and J. R. Brodeur. 1991. Intrinsic Germanium Gamma-Ray Data from the New American Petroleum Institute Spectral Gamma-Ray Calibration Models. WHC-SA-1176, Westinghouse Hanford Company, Richland, Washington. ABSTRACT: High-resolution gamma-ray spectra have been recorded at the new American Petroleum Institute (API) spectral gamma-ray logging calibration center with a U.S. Department of Energy passive spectral gamma-ray logging unit. These measurements were completed before gamma-ray source concentrations were assigned to the calibration model zones. Analyses of spectra gathered from five of the calibration zones yielded linear relationships between: 1) tentative uranium concentrations and intensities of the 352-, 609-, and 1,764- keV gamma-ray peaks and 2) tentative thorium concentrations and intensities of the 583-, 911-, and 2,615-keV gamma-ray peaks. The tentative potassium concentration and associated 1,461-keV gamma-ray peak intensity for the High Mix zone did not conform to the linear function defined by the tentative concentrations and peak intensities for the four other zones. The function implied that the peak intensity for the High Mix zone corresponded to a potassium concentration of about $4.05 \%$. This was substantially smaller than the tentative value of 5.62\%. A slightly different analysis led an API spectral gamma-ray calibration task group to concentrations of $3.98 \pm 0.26 \%$ for the High Mix zone and $0.90 \pm 0.12 \%$ for the Low Mix zone. The task group has recommended that the API assign these concentrations to the zones.

79. Koizumi, C. J., J. R. Brodeur, R. K. Price, J. E. Meisner, and D. C. Stromswold. 1994. HighResolution Gamma-Ray Spectrometry Logging for Contamination Assessment. Nuclear Geophysics 8:149-164.

80. Kos, S. E 1995. Assessment of Vadose Zone Radionuclide Contamination Around Single Shell Tank 241-C-103. WHC-SD-EN-TI-299, Rev. 0, Westinghouse Hanford Company, Richland, Washington.

81. Kos, S. E. 1995. Report of Spectral Gamma-Ray Surveys Acquired for the 100-UP-2 Project. WHC-SD-EN-TI-298, Rev. 0, Westinghouse Hanford Company, Richland, Washington. 
82. Kos, S. E. 1995. Report of Spectral Gamma-Ray Surveys Acquired for the 100-UP-1 Project. WHC-SD-EN-TI-297, Rev. 0, Westinghouse Hanford Company, Richland, Washington.

83. Last, G. V., K. R. Fecht, and M. A. Chamness. 1984. Scintillation Logging Plan for Monitoring Inactive Cribs. RHO-RE-PL-23, Rockwell Hanford Operations, Richland, Washington. ABSTRACT: This document presents a review of crib facilities, a description of the scintillation probe system, and a summary of previous studies; it also formulates a scintillation logging plan for monitoring the inactive crib facilities.

84. Lewis, R. E., and A. W. Pearson. 1992. Catalog of Borehole Geophysics for the 100 Areas and Adjacent 600 Area, Hanford Site, 1962--May 1992. A Research Report for Westinghouse Hanford Company. PNL-8230, Battelle Pacific Northwest Laboratories, Richland, Washington. ABSTRACT: This report catalogs geophysical borehole logs acquired between 1962, when logging began, and May 15, 1992, in the 100 Areas and the surrounding 600 Area of the Hanford Site. Separate tables were prepared for each respective set of wells. Each table lists all wells known to have been drilled, all borehole geophysical logs measured, and the location of these logs. No information is provided for logs acquired by the Westinghouse Hanford Company spectral gamma logging system. Maps are provided for identification of the specific wells in the 200 Areas. A cross reference between the well numbering scheme employed by the Basalt Waste Isolation Project and that based on the Hanford grid name is provided for the appropriate wells in the 600 area.

85. Lewis, R. E., and S. S. Teel. 1994. A Survey of Existing and Emerging Technologies for External Detection of Liquid Leaks at the Hanford Site. PNL-10176, Pacific Northwest Laboratory, Richland, Washington. ABSTRACT: This report presents a catalog of existing and emerging technologies that may be applicable for the detection of liquid leaks in the unsaturated subsurface external to storage and transfer facilities. The catalog was developed with the needs of the Hanford Site in mind, primarily those of tank farms. The needs for external leak detection for other Hanford facilities were also considered (e.g., retention basins, pipelines). A total of 29 technologies is described and rated in a spreadsheet provided with this report. These technologies can be divided into the following groups: 1) borehole geophysics, 2) surface geophysics,

3) borehole-to-borehole geophysics, 4) soil moisture instrumentation, 5) in situ sensors, 6) moisture removal and analysis, and 7) vapor extraction analysis. General descriptions of these groups and the evaluated technologies are provided in the text.

86. Lindberg, J. W., B. A. Williams, and F. A. Spane. 1997. Borehole Data Package for Well 69937-47A, PUREX Plant Cribs, CY 1996. PNNL-11515, Pacific Northwest National Laboratory, Richland, Washington. ABSTRACT: A new groundwater monitoring well (699-37-47A) was installed in 1996 as a downgradient well near the PUREX Plant Cribs Treatment, Storage, and Disposal Facility at Hanford. This document provides data from the well drilling and construction operations, as well as data from subsequent characterization of groundwater and sediment samples collected during the drilling process. The data include: well construction documentation, geologist's borehole logs, results of laboratory analysis of groundwater samples collected during drilling and of physical tests conducted on sediment samples collected during drilling, borehole geophysics, 
and results of aquifer testing including slug tests and flowmeter analysis. This well (699-37-47A) was constructed in support of the Hanford Federal Facility Agreement and Consent Order (TriParty Agreement) milestone M-24-00H and interim milestone M-24-35 (Ecology et al. 1994), and was funded under Project W-152.

87. Lockhead Martin Hanford Corporation. 1998. Analysis of Historical Gross Gamma Logging Data from BX Tank Farm. HNF-3531, Lockheed Martin Hanford Corporation, Richland, Washington.

88. Lockhead Martin Hanford Corporation. 1998. Analysis of Historical Gross Gamma Logging Data from TY Tank Farm. HNF-3831, Lockheed Martin Hanford Corporation, Richland, Washington.

89. MACTEC-ERS. 1997. Hanford Tank Farms Vadose Zone, Calibration Plan for Spectral Gamma-Ray Logging Systems. MAC-VZCP-1.7.3, Rev. 1 for U.S. Department of Energy, Grand Junction Projects Office, Grand Junction, Colorado.

90. MACTEC-ERS. 1997. Hanford Tank Farms Vadose Zone, Data Analysis Manual. MACVZCP 1.7.9, Rev. 1 for U.S. Department of Energy, Grand Junction Projects Office, Grand Junction, Colorado.

91. MACTEC-ERS. 1997. Hanford Tank Farms Vadose Zone, High Resolution Passive Spectral Gamma-Ray Logging Procedures. MAC-VZCP-1.7.10-1, Rev. 2 for U.S. Department of Energy, Grand Junction Projects Office, Grand Junction, Colorado.

92. MACTEC-ERS. 1997. Hanford Tank Farms Vadose Zone, Preventative Maintenance Procedures for the Spectral Gamma Logging System. MAC-VZCP-1.7.10.-2, Rev. 1 for U.S. Department of Energy, Grand Junction Projects Office, Grand Junction, Colorado.

93. MACTEC-ERS. 1997. Hanford Tank Farms Vadose Zone, Project Management Plan. MACVZCP-1.7.2, Rev. 1 for U.S. Department of Energy, Grand Junction Projects Office, Grand Junction, Colorado.

94. Menninga, C., and R. L. Brodzinski. 1981. Design Manual for a Well-Logging Probe Capable of Measuring Tritium. PNL-4069, Pacific Northwest Laboratory, Richland, Washington. ABSTRACT: The purpose of this instrument is to measure the concentration of tritium in situ in a well or bore hole. The instrument is designed to detect tritium at concentrations as low as terrestrial surface background. The instrument can sample air or water, and purify the sample from other radioactive nuclides and from chemical contaminants. The instrument will operate satisfactorily in the presence of a moderate gamma-ray background.

95. Mennings, C., and R. L. Brodzinski. 1983. Well-Logging Probe for Measuring Tritium: Construction and Operating Manual. PNL-4620, Pacific Northwest Laboratory, Richland, Washington. ABSTRACT: This document describes the as-built construction and operating 
procedures for a well-logging instrument capable of measuring tritium in situ in a well or borehole as small as 3-in. schedule 40 pipe. A companion document, A Design Manual for a Well-Logging Probe Capable of Measuring Tritium, PNL-4069, should be referred to for all design information and drawings. This document contains sections describing changes made between the design and construction phases, the general configuration of the instrument, and step-by-step operating procedures. The instrument can sample air or water and can purify the sample from other radionuclides or chemical contaminants. The instrument will operate satisfactorily in the presence of a moderate gamma-ray background and can measure tritium concentrations as low as $50 \mathrm{pCi} / \mathrm{ml}$ of water in normal logging operations.

96. Meisner, J. E. 1995. Vadose Zone Measurement Through Steel Casing Evaluation. WHCSD-EN-TI-304, Westinghouse Hanford Company, Richland, Washington. ABSTRACT: Westinghouse Hanford Company has initiated investigation into the use of a standard moisture gauge for measurement of in-formation moisture content from cased wells. The presence of air in the borehole does not destroy the instrument response; in fact, the response is very sensitive to formation moisture from steel-cased boreholes. Calibration of the prototype instrument configuration and some experimental characterization for various borehole constructions have been performed and reported. Recommendations for future study are also provided.

97. Meisner, J. E., R. K. Price, and R. R. Randall. 1996. Radionuclide Logging System In Situ Vadose Zone Moisture Measurement Calibration. WHC-SD-EN-TI-306, Westinghouse Hanford Company, Richland, Washington. ABSTRACT: Westinghouse Hanford Company has adapted a moisture gauge for use in the vadose zone logging of Hanford Site wells. A version of the instrumentation was previously evaluated and calibrated in March 1995. Since that time, nearly 50 wells have been logged. This report documents the calibration data and the results of various effects that influence or interfere with the response of count rate to formation moisture content.

98. Mercer, R. B. 1993. 1992 Borehole Completion Data Package for the Low-Level Burial Grounds. WHC-SD-EN-DP-049, Westinghouse Hanford Company, Richland, Washington. ABSTRACT: This report contains gross gamma-ray logs for ten new wells.

99. Mercer, R. B. 1993. 1991 Borehole Completion Data Package for the Low-Level Burial Grounds. WHC-SD-EN-DP-044, Westinghouse Hanford Company, Richland, Washington. ABSTRACT: Eighteen additional groundwater monitoring wells were installed around the lowlevel burial grounds in 1991. This data package contains information on the drilling, construction, development, and aquifer testing of these wells. This report contains gross gamma-ray logs for the wells.

100. Mercer, R. B. 1994. 1993 Borehole Completion Data Package for the Low-Level Burial Grounds. WHC-SD-EN-DP-086, Westinghouse Hanford Company, Richland, Washington. ABSTRACT: This report contains gross gamma-ray logs for two new wells. 
101. Mitchell, R. M., K. A. Bergstrom, C. J. Chou, D. L. Edwards, D. G. Horton, V. G. Johnson, B. M. Markes, T. H. Mitchell, R. K. Price, S. P. Reidel, K. D. Reynolds, and W. R. Thackaberry. 1998. TWRS Phase I Privatization Site Preconstruction Characterization Report. HNF-2067, Rev. 0, Fluor Daniel Hanford, Inc., Richland, Washington. ABSTRACT: The results of in situ gamma-ray and vadose zone moisture surveys for 15 existing wells and 5 temporary borings in the area of the former Grout Treatment Facility are presented.

102. Myers, D. A., D. L. Parker, MACTEC-ERS; G. W. Gee, V. G. Johnson, G. V. Last, and R. J. Serne. 1998. Lockheed Martin Hanford Corporation. Findings of the Extension of Borehole 41-09-39, 241-SX Tank Farm. HNF-2855, Pacific Northwest National Laboratory; and D. J. Moak, Waste Management Northwest for Lockheed Martin Hanford Corporation, Richland, Washington.

103. Narbutovskih, S. M., and F. Michelsen. 1994. Preliminary Results of a Seismic Borehole Test Using Downhole Shaped Charges at the DOE Hanford Site. WHC-SA-2304, Westinghouse Hanford Company, Richland, Washington. ABSTRACT: Geophysical site characterization studies can be important steps in the process of designing and monitoring remediation at hazardous waste storage facilities. Use of seismic techniques for subsurface characterization at the DOE Hanford Site has been limited. One reason is the lack of borehole velocity control, and lowvelocity sediments are highly attenuative. Consequently, standard techniques to provide velocity control are not adequate. Both Vertical Seismic Profiling and reversed VSP surveys are currently being investigated to provide velocity control and for subsurface imaging capabilities. Recently, a jet perforating gun was used to perforate a doubled-cased borehole in the 200 West Area. Acoustic emissions were recorded from numerous depths to obtain velocity control for a previous surface survey conducted in the same area. Both P- and S-wave data were recorded simultaneously from multiple horizons using the DAS-1 seismograph and 3-component geophones. The data were analyzed for a variety of uses besides velocity control. Signal attenuation was studied as a function of source depth and offset distance to evaluate formation absorption while vertical resolution was determined from the frequency spectrum. Preliminary results indicate that adequate P-wave velocity control can be obtained although the near-surface sediments are very attenuative. However, we conclude that the perforating gun produces little SH energy. Preliminary velocities indicate that reflection coefficients should be great enough to use surface techniques. Results from the frequency study suggest that a swept source for both surface and borehole surveys may be necessary to obtain required resolutions. Finally, signal attenuation as a function of formation facies suggests that seismic techniques may be useful in mapping perched water zones and for longterm vadose zone monitoring.

104. Narbutovskih, S. M., T. D. Halter, M. D. Sweeney, W. Daily, and A. L. Ramirez. 1996. Electrical Resistivity Tomography at the DOE Hanford Site. WHC-SA-3035-FP, Westinghouse Hanford Company, Richland, Washington. ABSTRACT: Recent work at the U.S. DOE Hanford site has established the potential of applying Electrical Resistivity Tomography (ERT) for early leak detection under hazardous waste storage facilities. Several studies have been concluded 
to test the capabilities and limitations of ERT for two different applications. First, field experiments have been conducted to determine the utility of ERT to detect and map leaks from underground storage tanks during waste removal processes. Second, the use of ERT for long-term vadose zone monitoring has been tested under different field conditions of depth, installation design, and acquisition mode/equipment and infiltration chemistry. This work involves transferring the technology from Lawrence Livermore National Laboratory (LLNL) to the Resource Conservation and Recovery Act (RCRA) program at the DOE Hanford Site. This paper covers field training studies relevant to the second application for long-term vadose zone monitoring.

105. Narbutovskih, S. M., D. F. Iwatate, M. D. Sweeney, A. L. Ramirez, W. Daily, R. M. Morey, and L. Christensen. 1996. Feasibility of CPT-Deployed Vertical Electrode Array in Single Shell Tank Farms. WHC-SD-EN-TA-004, Westinghouse Hanford Company, Richland, Washington.

106. Pacific Northwest National Laboratory (PNNL). 1999. Borehole Data Package for 1998 Wells Installed at Single-Shell Tank Waste Management Area T. PNNL-12125, Pacific Northwest National Laboratory, Richland, Washington. ABSTRACT: Two new Resource Conservation and Recovery Act (RCRA) groundwater monitoring wells were installed at the single-shell tank farm Waste Management Area (WMA) T in August through November of 1998 in fulfillment of Tri-Party Agreement (Ecology 1996) Milestone M-24-37. The wells are 299-W10-23 and 299W10-24. Well 299-W10-23 replaces well 299-W10-15 and well 299-W10-24 replaces well 299W10-27; both new wells are located north of WMA T and are downgradient monitoring wells. The locations of all wells in the extended monitoring network for WMA T are shown in Figure 1. The groundwater monitoring plan for WMA T (Caggiano and Goodwin 1991) describes the hydrogeology of the 200 West Area and WMA T. An Interim Change Notice to the groundwater monitoring plan provides justification for the new wells. The new wells were constructed to the specifications and requirements described in Washington Administrative Code (WAC) 173-160 and WAC 173-303. This document compiles information on the drilling and construction, well development pump installation, and sediment testing applicable to wells 299-W10-23 and 299W10-24. Appendix A contains copies of the geologist's log, the Well Construction Summary Report; and Well Summary Sheet (as-built diagram); Appendix B contains results of laboratory analyses of particle size distribution, $\mathrm{pH}$ conductivity, and moisture content; Appendix $\mathrm{C}$ contains geophysical logs analyses; and Appendix D contains analytical results from groundwater samples obtained during well construction. Aquifer tests (slug tests) were performed on both new wells after well completions. Results of the aquifer tests will be reported elsewhere. Additional documentation concerning well construction is on file with Bechtel Hanford, Inc., Richland, Washington.

107. Pacific Northwest National Laboratory (PNNL). 1999. Borehole Data Package for 1998 Wells Installed at Single-Shell Tank Waste Management Area U. PNNL-12126, Pacific Northwest National Laboratory, Richland, Washington. ABSTRACT: Two new Resource Conservation and Recovery Act (RCRA) groundwater monitoring wells were installed at the single-shell tank farm Waste Management Area (WMA) U in October 1998 in fulfillment of Tri-Party Agreement 
(Ecology 1996) milestone M-24-39. The wells are 299-W19-41 and 299-W19-42. Well 299-W1941 is located east of the southeastern corner of the WMA and replaces downgradient well 299W19-32. Well 299-W19-42 is located east of the WMA near the northeastern comer and is a new downgradient monitoring well. The locations of all wells in the monitoring network are shown on Figure 1. The groundwater monitoring plan for WMA U (Caggiano and Goodwin 1991) describes the hydrogeology of the 200 West Area and WMA U. An Interim Change Notice to the groundwater monitoring plan provides justification for the new wells. The new wells were constructed to the specifications and requirements described in Washington Administrative Code (WAC) 173-160 and WAC 173-303. This document compiles information on the drilling and construction; well development pump installation; and sediment testing applicable to wells 299-W19-41 and 299W19-42. Appendix A contains the geologist's log, the well construction summary report, and well summary sheet; Appendix B contains results of laboratory measurements of particle size distribution $\mathrm{pH}$ conductivity, and calcium carbonate and moisture contents; and Appendix C contains geophysical logs. Aquifer tests (slug tests) were performed on both new wells. Results from the aquifer tests will be reported elsewhere. Additional documentation concerning well construction is on file with Bechtel Hanford, Inc., Richland, Washington.

108. Pacific Northwest National Laboratory (PNNL). 1999. Borehole Data Package for Well 299-E33-44 at Single-Shell Tank Waste Management Area B-BX-BY. PNNL-12128, Pacific Northwest National Laboratory, Richland, Washington. ABSTRACT: One new Resource Conservation and Recovery Act (RCRA) groundwater monitoring well was installed during September 1998 at the single-shell tank farm Waste Management Area (WMA) B-BX-BY. The well is 299-E33-44 and is located east of the BY single-shell tank farm. The well is a new upgradient monitoring well drilled in support of the groundwater assessment program at WMA B-BX-BY. This document is a compilation of information on the drilling and construction well development pump installation and sediment testing and analyses applicable to well 299-E33-44. Appendix A contains copies of the geologist's log, the Well Construction Summary Report, and Well Summary Sheet (as-built diagram); Appendix B contains results of Laboratory analyses completed on samples of sediment from the well; and Appendix $\mathrm{C}$ contains geophysical logs. An aquifer test (slug test) was done in the well after well completion. Results from the aquifer test will be published elsewhere. Additional documentation concerning well construction is on file with Bechtel Hanford Inc., Richland, Washington.

109. Pacific Northwest National Laboratory (PNNL). 1999. Borehole Data Package for 1998 Wells Installed at Single-Shell Tank Waste Management Area TX-TY. PNNL-12124, Pacific Northwest National Laboratory, Richland, Washington. ABSTRACT: Four new Resource Conservation and Recovery Act (RCRA) groundwater monitoring wells were installed at the single-shell tank farm Waste Management Area (WMA) TX-TY during August through November of 1998 in fulfillment of Tri-Party Agreement (Ecology 1996) milestone M-24-38. The wells are 299-W1026, 299- W14-13, 299-W14-14, and 299-W15-40. Well 299-W10-26 is located outside the east fence of the TY tank farm and replaces downgradient well 299-W10-18; well 299-W14-13 is located along the east fence near the northeast corner of the TX tank farm and replaces downgradient well 299-W10-18; well 299-W14-13 is located outside the east fence in the south half of the TX tank farm and is a new downgradient well; and well 299-W15-40 is located on the west side 
of the TX tank farm and is a new upgradient well. The locations of all wells in the monitoring network are shown on Figure 1. The groundwater monitoring plan for WMA TX-TY (Caggiano and Goodwin 1991) describes the hydrogeology of the 200 West Area and WMA TX-TY. An Interim Change Notice to the groundwater monitoring plan provides justification for the new wells. The new wells were constructed to the specifications and requirements described in Washington Administrative Code (WAC) 173-160 and WAC 173-303. This document compiles information on the drilling and construction, well development pump installation; groundwater sampling; and sediment testing applicable to wells 299-W10-26, 299-W14-13, 299- W14-14, and 299-W15-40. Appendix A contains the geologist's log, the Well Construction Summary Report, and Well Summary Sheet (as-built diagram); Appendix B contains results of laboratory analyses of particle size distribution, $\mathrm{pH}$ conductivity, calcium carbonate content; major cation and anion concentrations from 1:1 water, sediment extracts, and moisture content; Appendix $\mathrm{C}$ contains geophysical logs; and Appendix D contains the analytical results from groundwater samples obtained during well construction. Aquifer tests (slug tests) were performed on all the new wells after well completion. Results of the aquifer tests will be reported elsewhere. Additional documentation concerning well construction is on file with Bechtel Hanford, Inc., Richland, Washington.

110. Petrie, G. M., D. Gibson, and S. C. Blair. 1983. User's Manual for Geophysical Well-Logging Software Programs. PNL-4562, Pacific Northwest Laboratory, Richland, Washington. ABSTRACT: Since 1958, the Groundwater Surveillance Program for the Hanford Site has made geophysical logging measurements in most of the 800 wells and deep boreholes that have been drilled on the Hanford Site. In 1980 the Pacific Northwest Laboratory (PNL), which conducts the Groundwater Surveillance Program, began forming a computerized database for storing and retrieving geophysical well log data and developing software for quantitative analysis of the well $\log$ data. This report, designed to serve as a user's guide, documents the database system that handles the well log data. Two programs, DIGLOG1 and LOGIT, are used to manipulate the data. The program DIGLOG1 translates analog paper strip charts into digital format; the program LOGIT is a general utility program that edits, displays, checks, stores, writes, and deletes sets of well log data. These two programs do not provide sophisticated display and analytical capabilities; rather, they provide programs that give the user easy access to powerful standard analytical software.

111. Price, R. K. 1992. "Preliminary Evaluation of RLS Log Surveys for Boreholes 299-W19-11 and 299-W22-75." Westinghouse Hanford Company Internal Memo, 81230-92-006 to M. J. Galgoul. ABSTRACT: Memo transmits logs from the 216-U-1 and 216-U-12 cribs.

112. Price, K. R. 1992. Spectral Gamma-Ray Log Report for 200-BP-1 Borehole Surveys. WHC-SD-EN-ES-029, Rev. 0, Westinghouse Hanford Company, Richland, Washington.

113. Price, R. K. 1993. Spectral Gamma-Ray Log Report for Radionuclide Surveys Acquired for 100-NR-1 Operable Unit. WHC-SD-EN-TI-189, Rev. 0, Westinghouse Hanford Company, Richland, Washington.

114. Price, K. R. 1993. Spectral Gamma-Ray Log Report for Tank 241-T-101 Borehole Surveys. WHC-SD-EN-TI-163, Rev. 0, Westinghouse Hanford Company, Richland, Washington. 
115. Price, R. K. 1993. Spectral Gamma-Ray Log Report for 100 Area Borehole Surveys. WHC-SD-EN-TI-123, Rev. 0, Westinghouse Hanford Company, Richland, Washington.

116. Price, R. K. 1994. Radionuclide Logging of Tank 241-T-106, Borehole 299-W10-196. WHC-SD-EN-TI-271, Rev. 0, Westinghouse Hanford Company, Richland, Washington.

ABSTRACT: Geophysical surveys were conducted in borehole 299-W10-196 during construction to profile the radionuclide contaminants in the vadose zone that leaked from single-shell tank 216T-106. Contaminants from the tank leak were detected to 120 feet below the ground surface. A second complete geophysical survey was conducted about one year after drilling was completed to evaluate contaminant movement that may have been caused by the presence of the cased borehole. Contaminant movement would warrant plugging and abandoning the borehole. Comparison of the two surveys indicate that no contamination movement to greater depths can be identified.

117. Price, R. K. 1995. Vadose Zone Monitoring Plan Using Geophysical Nuclear Logging for Radionuclides Discharged to Hanford Liquid Waste Disposal Facilities. WHC-SD-EN-AP-087, Westinghouse Hanford Company, Richland, Washington. ABSTRACT: During plutonium production at Hanford, large quantities of hazardous and radioactive liquid effluent waste have been discharged to the subsurface (vadose zone). These discharges at over 330 liquid effluent disposal facilities (i.e., cribs, ditches, and ponds) account for over 3,000,000 curies of radioactive waste released into the subsurface. It is estimated that $10 \%$ of the contaminants have reached the groundwater in many places. Continuing migration may further impact groundwater quality in the future. Through the RCRA Operational Monitoring Program, a Radionuclide Logging System (RLS) has been obtained by Hanford Technical Services (HTS) and enhanced to measure the distribution of contaminants and monitor radionuclide movement in existing groundwater and vadose zone boreholes. Approximately 100 wells are logged by HTS each year in this program. In some cases, movement has been observed years after discharges were terminated. A similar program is in place to monitor the vadose zone at the Tank Farms. This monitoring plan describes Hanford Programs for monitoring the movement of radioactive contamination in the vadose zone. Program background, drivers, and strategy are presented. The objective of this program is to ensure that DOE-RL is aware of any migration of contaminants in the vadose zone, such that groundwater can be protected and early actions can be taken as needed.

118. Price, R. K. 1996. Evaluation of Historical Dry Well Surveillance Logs. WHC-SD-ENVTI-001 Rev. 0, Westinghouse Hanford Company, Richland, Washington. ABSTRACT: Several dry well surveillance logs from 1975 through 1995 for the SX tank farm have been examined to identify potential subsurface zones of radioactive contaminant migration. Several dynamic conditions of the gamma-ray-emitting radioactive contaminants have been identified.

119. Price, R. K. 1997. “In Situ Spectral Gamma-Ray Survey Results of Auger Drilled Boreholes on East Side of 221-B Plant in Support of W-059 Soil Sampling." Waste Management Federal Services, Inc., Northwest Operations Letter, WMNW-9751307 to D. K. Smith. ABSTRACT: B\&W Hanford Company requested auger drilling and high-purity germanium 
(HPGe) spectral gamma-ray analysis of the radionuclide distribution and concentration of the sediments on the south side of 221-B to a depth of $1.22 \mathrm{~m}(4 \mathrm{ft})$. The results of the survey are presented.

120. Ramirez, A., W. Daily, A. Binley, and D. LaBrecque. 1996. Tank Leak Detection Using Electrical Resistance Methods. UCRL-JC-122875, Lawrence Livermore National Laboratory, California. ABSTRACT: Large volumes of hazardous liquids and high-level radioactive wastes are stored worldwide in surface and underground tanks. Frequently these tanks are found to leak, thereby resulting in not only a loss of stored inventory, but in contamination to soils and groundwater. It is important to develop a reliable method of detecting leaks before large quantities are emitted into the environment surrounding the tanks. Two field experiments were performed to evaluate the performance of electrical resistance tomography (ERT) as a leak detection method under metal underground storage tanks (UST). This paper provides a summary of the field experiments performed under a 15-m-diameter steel tank mockup located at the Hanford Reservation.

121. Randall, R. R. 1994. Calibration of the Radionuclide Logging System Germanium Detector. WHC-SD-EN-TI-292, Westinghouse Hanford Company, Richland, Washington.

ABSTRACT: Gamma-ray source concentrations are derived from log data by calculations that employ the calibration factors and correction functions described in this report. Calibration data were collected with a Radionuclide Logging System. Analyses of the calibration data established: 1) calibration factors for potassium, uranium, and thorium and 2) a calibration function that permits assessments of cesium-137, cobalt-60, and other artificial nuclides not represented in the calibration models.

122. Randall, R., and D. Stromswold. 1995. Procedures for Calibrating Scintillation Gamma-Ray Well Logging Tools Using Hanford Formation Models. WHC-SD-EN-TI-293, Westinghouse Hanford Company, Richland, Washington.

123. Randall, R. R., and R. K. Price. 1998. Analysis Techniques Applied to the Dry Well Surveillance Gross Gamma Ray Data at the SX Tank Farm. WMNW/TRS-ES-VZMA-001, Waste Management Federal Services, Inc., Northwest Operations, Richland, Washington.

124. Randall, R. R., and R. K. Price. 1998. Monitoring Results for the SX Single Shell Tank Farm Dry Well Gamma Ray Surveillance Log Surveys. WMNW/TRS-ES-VZMA-002, Waste Management Federal Services Inc., Northwest Operations, Richland, Washington.

125. Raymond, J. R. 1962. A Well Water Temperature Logging System. HW-73994, General Electric Company, Richland, Washington.

126. Raymond, J. R., and V. L. McGhan. 1964. Scintillation Probe Results - 200 Area Waste Disposal Site Monitoring Wells. HW-84577, General Electric Company, Richland, Washington. ABSTRACT: This report describes scintillation probe results from the 200 Area waste disposal site monitoring wells over the period of 1953 to 1963 . The 1953 and 1954 data were from 
scattered probings made during equipment development tests. General scintillation probing of essentially all 200 area monitoring wells was done in 1958, 1959, and 1963.

127. Raymond, J. R. 1964. A Gamma Scintillation Well Probe System. HW-81972, General Electric Company, Richland, Washington.

128. Raymond, J. R., and V. L. McGhan. 1992. History of Geophysical Well Logging on the Hanford Site, unpublished letter report, Pacific Northwest Laboratory, Richland, Washington.

129. Reeder, P. L., D. C. Stromswoldl, R. L. Brodzinski, J. H. Reeves, and W. E. Wilson. 1995. Moisture Measurement for High-Level-Waste Tanks Using Copper Activation Probe in Cone Penetrometer. PNL-10837, Pacific Northwest Laboratory, Richland, Washington.

ABSTRACT: Laboratory tests have established the feasibility of using neutron activation of copper as a means for measuring the moisture in Hanford's high-level radioactive waste tanks. The performance of the neutron activation technique to measure moisture is equivalent to the neutron moisture gauges or neutron logs commonly used in commercial well-logging. The principal difference is that the activation of ${ }^{64} \mathrm{Cu}\left(\mathrm{t}_{1 / 2}=12.7 \mathrm{~h}\right)$ replaces the neutron counters used in moisture gauges or neutron logs. For application to highly radioactive waste tanks, the $\mathrm{Cu}$ activation technique has the advantage in that it is insensitive to very strong gamma radiation fields or high temperatures. In addition, this technique can be deployed through tortuous paths or in confined spaces such as within the bore of a cone penetrometer. However, the results are not available in "real time." The copper probe's sensitivity to moisture was measured using simulated tank waste of known moisture content. This report describes the preparation of the simulated waste mixtures and the experiments performed to demonstrate the capabilities of the neutron activation technique. These experiments included determination of the calibration curve of count rate versus moisture content using a single copper probe, measurement of the calibration curve based on "nearfield" to "far-field" counting ratios using a multiple probe technique, and profiling the activity of the copper probe as a function of the vertical height within a simulated waste barrel.

130. Reeves, J. H., R. J. Arthur, R. L. Brodzinske, and C. L. Shepard. 1995. Tube-Excited X-Ray Fluorescence Spectrometer for Use in Small-Diameter Boreholes. PNL-SA-23193, Pacific Northwest Laboratory, Richland, Washington. ABSTRACT: A portable in situ X-ray fluorescence analytical system that uses an x-ray tube excitation source and a cooled $\mathrm{Si}(\mathrm{Li})$ spectrometer for detecting characteristic emission $\mathrm{x}$ rays has been developed for use in small-diameter wells and boreholes. The 15-watt, iron-anode x-ray tube operates up to $30 \mathrm{kV}$. Three wells at the Sandia National Laboratory Chemical Waste Landfill, lined with 76 microns thick polyethylene, were logged specifically for $\mathrm{Cr}$ contamination. Detection limits below $50 \mathrm{ppM}$ were achieved with counting intervals of 600 seconds and with the $\mathrm{Si}(\mathrm{Li})$ detector operating at $450-\mathrm{eV}$ resolution (full width at half maximum (FWHM) for the Mn K-alpha x ray).

131. Reidel, S. P., K. D. Reynolds, and D. G. Horton. 1998. Immobilized Low-Activity Waste Site Borehole 299 E17-21. PNNL-11957, Pacific Northwest National Laboratory, Richland, Washington. 
132. Rohay, V. J., and D. C. Weekes. 1998. Borehole Summary Report for the 216-B-2-2 Ditch. BHI-01177, Bechtel Hanford, Inc., Richland, Washington. ABSTRACT: The purpose of this borehole summary report is to provide the results of the drilling and sampling as a data package. The 216-B-2-2 Ditch borehole data will be evaluated with other existing data during remedial investigation/feasibility study (RI/FS) work plan development for the Gable Mountain Pond/ B-Pond and Ditches Cooling Water Group (200-CW-1). The document contains natural gamma, spectral gamma, neutron moisture, and gross gamma logs.

133. Routson, R. C., W. H. Price, D. J. Brown, and K. R. Fecht. 1979. High-Level Waste Leakage from the 241-T-106 Tank at Hanford. RHO-ST-14, Rockwell Hanford Operations, Richland, Washington.

134. Rust Geotech. 1995. Vadose Zone Characterization Project at the Hanford Tank Farms, Spectral Gamma-Ray Borehole Geophysical Logging Characterization and Baseline Monitoring Plan for the Hanford Single-Shell Tanks. P-GJPO-1786 for U.S. Department of Energy, Grand Junction Projects Office, Grand Junction, Colorado.

135. Rust Geotech. 1995. Vadose Zone Characterization at the Hanford Tank Farms, HighResolution Passive Spectral Gamma-Ray Logging Procedures. P-GJPO-1783, Rev. 1 for U.S. Department of Energy, Grand Junction Projects Office, Grand Junction, Colorado.

136. Rust Geotech. 1995. Vadose Zone Characterization Project at the Hanford Tank Farms, Calibration of Two Spectral Gamma-Ray Logging Systems for Baseline Characterization Measurements in the Hanford Tank Farms. GJPO-HAN-1 for U.S. Department of Energy, Grand Junction Projects Office, Grand Junction, Colorado.

137. Rust Geotech. 1996. Vadose Zone Characterization Project at the Hanford Tank Farms, Biannual Recalibration of Two Spectral Gamma-Ray Logging Systems Used for Baseline Characterization Measurements in the Hanford Tank Farms. GJPO-HAN-3 for U.S. Department of Energy, Grand Junction Projects Office, Grand Junction, Colorado.

138. Rust Geotech. 1996. Second Biannual Recalibration of Two Spectral Gamma-Ray Logging Systems Used for Baseline Characterization Measurements in the Hanford Tank Farms. GJPOHAN-5 for U.S. Department of Energy, Grand Junction Office, Grand Junction, Colorado. ABSTRACT: The U.S. Department of Energy's (DOE) Grand Junction Project Office (GJPO) is establishing an initial, or baseline, characterization of gamma-ray-emitting contaminants in the subsurface of the Tank Farms at the DOE Hanford Site in Washington State. The baseline data are gathered by logging existing monitoring boreholes with two high-resolution passive spectral gamma-ray logging systems (SGLSs), informally known as Gamma 1 and Gamma 2. The project document, "Vadose Zone Monitoring Project at the Hanford Tank Farms, Spectral Gamma-Ray Borehole Geophysical Logging Characterization and Baseline Monitoring Plan for the Hanford Single-Shell Tanks," specifies that both systems must be recalibrated using the calibration standards at the Hanford borehole logging calibration center every 6 months. DOE presents a description of the first recalibrations. 
139. Schilk, A. J., R. W. Perkins, K. H. Abel, and R. L. Brodzinski. 1993. Surface and Subsurface Characterization of Uranium Contamination at the Fernald Environmental Management Site. PNL-8617, Pacific Northwest Laboratory, Richland, Washington. ABSTRACT: The past operations of uranium production and support facilities at several Department of Energy (DOE) sites have occasionally resulted in the local contamination of some surface and subsurface soils, and the three-dimensional distribution of the uranium at these sites must be thoroughly characterized before any effective remedial protocols can be established. To this end, Pacific Northwest Laboratory (PNL) has been tasked by the DOE's Office of Technology Development with adapting, developing, and demonstrating technologies for the measurement of uranium in surface and subsurface soils at the Fernald Uranium in Soils Integrated Demonstration site. These studies are detailed in this report.

140. Schilk, A. J., R. W. Perkins, K. H. Abel, R. L. Brodzinski, and Brown. 1993. Quantitative In Situ Uranium Contaminant Mapping Using High-Resolution Gamma-Ray Spectrometry and Beta Scintillation Counting. PNL-SA-23141, Pacific Northwest Laboratory, Richland, Washington. ABSTRACT: Traditional means of measuring radionuclide concentrations in soils over large areas are often time-consuming, cumbersome, expensive, and non-representative. In developing new methods for the real-time characterization of large-scale uranium contamination, three original and/or adapted technologies were demonstrated at the Fernald Environmental Management Project near Cincinnati, Ohio. The use of collimated downwell gamma-ray spectrometry, in situ surface gamma-ray spectrometry, and a novel high-energy beta scintillation sensor represents significant improvements over, and largely eliminates the need for, the collection of myriad soil samples and subsequent laboratory analyses. The results from a preliminary screening of two sites within the Fernald facility are presented here, and future versions of the beta sensor and surface gamma spectrometer are discussed in some detail.

141. Sheen, E. M., W. L. Bunch, and M. R. Wood. 1964. Neutron Soil Moisture Monitor. HW-82009, General Electric Company, Richland, Washington.

142. Singleton, K. M., and K. A. Lindsey. 1994. Groundwater Impact Assessment Report for the 216-U-14 Ditch. WHC-EP-0698, Westinghouse Hanford Company, Richland, Washington. ABSTRACT: Appendix H contains 8 borehole surveys obtained with the spectral gamma-ray logging truck in the vicinity of the U-14 ditch. The maximum detected activity from man-made radionuclides did not exceed $5 \mathrm{pCi} / \mathrm{g}$ and the activity occurred near the ground surface in all wells. The appendix also contains gross gamma logs from the same 8 boreholes.

143. Smith, R. M., D. J. Bates, and R. E. Lundgren. 1989. Resource Conservation and Recovery Act Groundwater Monitoring Projects for Hanford Facilities: Progress Report, July 1September 30, 1989. Volume 1. Text. Volume 2. Appendices. PNL-7222, Vols. 1 and 2, Pacific Northwest Laboratory, Richland, Washington. ABSTRACT: This is Volume 1 of a twovolume document that describes the progress of 14 Hanford Site groundwater monitoring projects for the period July 1 to September 30, 1989. This volume discusses the projects; Volume 2 provides as-built diagrams, completion/inspection reports, drilling logs, and geophysical logs for wells drilled, completed, or logged during this period. Volume 2 can be found on microfiche in the back 
pocket of Volume 1. The work described in this document is conducted by the Pacific Northwest Laboratory under the management of Westinghouse Hanford Company for the U.S. Department of Energy. Concentrations of groundwater constituents are compared to federal drinking water standards throughout this document for reference purposes. All drinking water supplied from the sampled aquifer meets regulatory standards for drinking water quality.

144. Smith, R. M., D. J. Bates, and R. E. Lundgren. 1990. Resource Conservation and Recovery Act Groundwater Monitoring Projects for Hanford Facilities: Progress Report, October 1December 31, 1989. Volume 2, Appendixes. PNL-7306 Vol. 2, Pacific Northwest Laboratory, Richland, Washington. ABSTRACT: This Volume 2 of a two-volume set of documents that describes the progress of 15 Hanford Site groundwater monitoring projects for the period October 1 to December 31, 1989. This volume provides well completion information to supplement information included in the previous quarterly report and as-built diagrams, well completion/inspection reports, drilling logs, and geophysical logs for wells drilled during this quarter near the single-shell tanks.

145. Spear, W. G., and M. O. Rankin. 1956. A Scintillation Well-Logging System. HW-39273, General Electric Company, Richland, Washington.

146. Strait, S. R., and B. A. Moore. 1982. Geohydrology of the Rattlesnake Ridge Interbed in the Gable Mountain Pond Area. RHO-ST-38, Rockwell Hanford Operations, Richland, Washington. ABSTRACT: Liquid waste disposal practices at the U.S. Department of Energy's Hanford Site include the discharge of low-level radioactive liquid waste to cribs, trenches, and surface ponds. A study was conducted to develop a conceptual groundwater flow model and characterize the current distribution of radionuclides in the groundwater of the Rattlesnake Ridge interbed at one of these facilities, the Gable Mountain Pond (facility 216-A-25). Specially constructed wells were drilled in the vicinity of Gable Mountain Pond to obtain radionuclide distribution data and hydrologic properties of the aquifer. The acquired data were analyzed to determine concentrations of anions, cations, selected radionuclides, and groundwater flow patterns. Geologic mechanisms, which may be responsible for the determined concentration distribution, were evaluated using borehole and subsurface geophysical techniques.

147. Stromswold, D. C. 1994. Calibration Facilities for Borehole and Surface Environmental Radiation Measurements. PNL-SA-23149, Battelle Pacific Northwest Laboratories, Richland, Washington. ABSTRACT: Measuring radiation from contaminated soil and buildings is important in the cleanup of land areas and facilities. It provides the means for quantifying the amount of contamination and assessing the success of efforts to restore areas to acceptable conditions for public use. Instruments that measure in situ radiation from natural or radiochemically contaminated earth formations must be calibrated in appropriate facilities to provide quantitative assessments of concentrations of radionuclides. For instruments that are inserted into boreholes, these calibration facilities are typically special models having holes for probe insertion and having sufficient size to appear radiometrically "infinite" in extent. The U.S. Department of Energy (DOE) has such models at Hanford, Washington, and Grand Junction, Colorado. They are concrete cylinders having a central borehole and containing known, enhanced 
amounts of K, U, and Th for spectral gamma-ray measurements. Additional models contain U for calibrating neutron probes for fissile materials and total-count gamma-ray probes. Models for calibrating neutron probes for moisture measurements in unsaturated formations exist for steelcased boreholes at Hanford and for uncased boreholes at the DOE's Nevada Test Site. Large surface pads are available at Grand Junction for portable, vehicle-mounted, or airplane-mounted spectral gamma-ray detectors.

148. Stromswold, D. C. 1994. Calibration Facilities at Hanford for Gamma-Ray and FissionNeutron Well Logging. PNL-9958, Battelle Pacific Northwest Laboratories, Richland, Washington. ABSTRACT: Well-logging tools that detect gamma rays emitted from earth formations need to be calibrated in appropriate facilities to provide quantitative assessments of concentrations of radionuclides based on detected gamma rays. These facilities are typically special models having a hole to insert tools and having sufficient physical size to simulate actual earth formations containing known amounts of radionuclides. The size, generally 3 to 5 feet in diameter and 4 to 6 feet tall, is such that the source of radiation appears infinite in extent to a tool detecting the radiation inside the model. Such models exist at Hanford as concrete cylinders having a central borehole and containing known, enhanced amounts of K, U, and Th. Data collected in these models allow calibration of the logging system to measure radionuclide concentrations in formations around boreholes in the field. The accuracy of the calculated field concentrations depends on the correctness of the original calibration, the statistical precision of the data, and the similarity of the logging conditions to the calibration conditions. Possible methods for analyzing the data collected in the calibration facilities are presented for both spectral and total-count gammaray systems. Corrections are typically needed for the effects of steel casing in boreholes and the presence of water rather than air in the holes. Data collected in the calibration models with various steel casings and borehole fluids allow such correction factors to be determined.

149. Stromswold, D. C. 1994. Technical Evaluation of Software for Gamma-Ray Logging System. PNL-9807, Pacific Northwest Laboratory, Richland, Washington. ABSTRACT: This report contains results of a technical review of software, identified as LGCALC, that processes data collected by high-resolution gamma-ray borehole logging system. The software presently operates with Westinghouse Hanford Company, Department of Geosciences, to process data collected by the Radionuclide Logging System. The software has been reviewed for its suitability for processing data to be collected by new high-resolution gamma-ray logging trucks scheduled to begin operational tests within Westinghouse Tank Waste Remediation Systems during 1994.

150. Sweeney, M. D. 1993. Borehole Completion Report for the 200 Areas Treated Effluent Disposal Basin - Project W-049H. WHC-SD-EN-DP-068, Westinghouse Hanford Company, Richland, Washington. ABSTRACT: This data package contains gross gamma-ray logs for three new wells.

151. Swanson, L. C. 1992. Borehole Completion Data Package for Grout Treatment Facility Well 299-E25 39. WHC-SD-EN-DP-048, Westinghouse Hanford Company, Richland, Washington. ABSTRACT: This report contains a gross gamma-ray log for the new well. 
152. Swanson, L. C. 1994. Borehole Completion Data Package, Grout Treatment Facility Wells 299-E25-49, 299-E25-50, and 299-E25-1000. WHC-SD-EN-DP-085, Westinghouse Hanford Company, Richland, Washington. ABSTRACT: This report contains spectral gamma-ray and gross gamma-ray logs for the new wells.

153. Szwartz, G. J. 1996. Spectral Gamma-Ray Logging Report for the 216-B-57 Crib Groundwater Impact Assessment. WHC-SD-EN-TI-257, Rev. 0, Westinghouse Hanford Company, Richland, Washington.

154. Szwartz, G. J. 1996. Spectral Gamma-Ray Logging Report for the Six New Characterization Boreholes in the 100-FR-1 Operable Unit. WHC-SD-EN-TI-258, Rev. 0, Westinghouse Hanford Company, Richland, Washington.

155. Teel, S. S., R. E. Lewis, M. A. Chamness, D. C. Lanigan, V. L. McGhan, and R. J. Brockman. 1992. B-Plant Aggregate Area Management Study Geologic Data Package. WHC-SD-EN-TI026, Westinghouse Hanford Company, Richland, Washington. ABSTRACT: This data package includes all of the geologic and geophysical information requested by the Westinghouse Hanford Company for the B-Plant Aggregate Area. This information includes a listing of all of the boreholes within this aggregate area as defined by the Westinghouse Hanford Company and amended by the Pacific Northwest Laboratory. Geophysical data (Table 1) includes the type of log, date, and interval logged for each of the above wells, as well as the location where these data are stored. Table 2 includes the types of geologic data available for each of these wells (i.e., driller's $\log$, geologist's $\log$ ) and any other related data (sieve, $\mathrm{CaCO}_{3}$ content, moisture content, and chemical analyses). Table 3 provides a summary of the well construction information for each well including drill depth, completion depth, screened interval, and coordinates.

156. Tillson, D. D., and V. L. McGhan. 1969. Changes in Scintillation Probe Findings - 1963 to 1968, 200 Area Waste Disposal Site Monitoring Wells. BNWL-CC-2255, Pacific Northwest Laboratory, Richland, Washington. ABSTRACT: This report provides a semiquantitative evaluation of the magnitude and extent of subsurface contamination beneath and adjacent to facilities for ground disposal of liquid radioactive wastes. Also, this study provided additional information on downward migration rates of radiocontaminants in the subsoils at disposal facilities that have been out of service for a long time.

157. U.S. Department of Energy. 1996. Vadose Zone Characterization Project at the Hanford Tank Farms: SX Tank Farm Report. GJPO-HAN-4, U.S. Department of Energy, Grand Junction Office, Grand Junction, Colorado. ABSTRACT: The SX Tank Farm is located in the southwest portion of the 200 West Area of the Hanford Site. This tank farm consists of 15 single-shell tanks (SSTs), each with an individual capacity of 1 million gallons (gal). These tanks currently store high-level nuclear waste that was primarily generated from what was called the oxidation-reduction or "REDOX" process at the S-Plant facility. Ten of the 15 tanks are listed in Hanlon as "assumed leakers" and are known to have leaked various amounts of high-level radioactive liquid to the vadose zone sediment. The current liquid content of each tank varies, but the liquid from known leaking tanks has been removed to the extent possible. In 1994, the U.S. Department of Energy 
Richland Office (DOE-RL) requested the DOE Grand Junction Projects Office (GJPO), Grand Junction, Colorado, to perform a baseline characterization of contamination in the vadose zone at all the SST farms with spectral gamma-ray logging of boreholes surrounding the tanks. The SX Tank Farm geophysical logging was completed and the results of this baseline characterization are presented in this report.

158. U.S. Department of Energy. 1994. Limited Field Investigation Report for the 100-HR-3 Operable Unit. DOE/RL-93-43, Richland Operations Office, Richland, Washington.

ABSTRACT: This limited field investigation (LFI) was conducted to assess the applicability of interim remedial measures (IRM) for reducing human health and environmental risks within the 100-HR-3 Groundwater Operable Unit. The 100-HR-3 Operable Unit comprises three subareas; the $100 \mathrm{D}$ Area, the $100 \mathrm{H}$ Area, and those portions of the 600 Area between the two reactor areas. The operable unit is one of seven operable units associated with the $100 \mathrm{D}$ and $\mathrm{H}$ Areas. Operable units 100-DR-1, 100-DR-2, 100-DR-3, 100-HR-1, 100-HR-2, and 100-IU-4 address contaminant sources while 100-HR-3 addresses contamination present in the underlying groundwater. The primary method of field investigation used during this LFI was the installation and sampling of monitoring wells. Samples were collected from the groundwater and soils and submitted for laboratory analysis. Boreholes were surveyed for radiological contamination using downhole geophysical techniques to further delineate the locations and levels of contaminants. All samples were screened to ascertain the presence of volatile organic compounds and radionuclides. Analytical data were subjected to validation; all of round one, two, and three, and a minimum of $10 \%$ of round four data associated with the LFI were validated. A screening method was used to identify contaminants of potential concern (COPC). This screening method eliminated constituents that were below background levels from further consideration. Constituents that are considered non-toxic to humans were eliminated from the human health evaluation. Data consistency and blank contamination were also evaluated in the screening process. The COPC was then evaluated further in the qualitative risk assessment (QRA). A human health QRA was performed using conservative (maximum equilibrated contaminant levels from the LFI) analyses.

\section{U.S. Department of Energy. 1994. Limited Field Investigation Report for the 100-KR-4 Operable Unit. DOE/RL-93-79, Richland Operations Office, Richland, Washington.}

ABSTRACT: This limited field investigation (LFI) was conducted to optimize the use of interim remedial measures (IRM) for expediting cleanup while maintaining a technically sound and costeffective program. The 100-KR-4 Operable Unit is one of four operable units associated with the $100 \mathrm{~K}$ Area. Operable units KR-1, KR-2, and KR-3 address contaminant sources while 100-KR-4 addresses contamination present in the underlying groundwater. The IRM decision process for groundwater operable units is based on three aspects: 1) Is the concentration greater than Hanford background? 2) Does the concentration present a medium or high human-health risk? and 3) Does the concentration exceed an ecologically based applicable, relevant, and appropriate requirements (ARAR) or present an environmental hazard quotient? The primary methods of investigation used during this LFI were the installation of monitoring wells and sampling of groundwater. The samples collected from the groundwater and soils were submitted for laboratory analysis. Boreholes were surveyed for radiological contamination using downhole geophysical techniques to further delineate the location and degree of contamination. All soil samples were screened to ascertain the 
presence of volatile organic compounds and radionuclides. Analytical data were subjected to validation; all first round and a minimum of $10 \%$ of subsequent round data were validated.

160. U.S. Department of Energy. 1994. Limited Field Investigation Report for the 100-BC-5 Operable Unit. DOE/RL-93-37, Richland Operations Office, Richland, Washington.

ABSTRACT: This limited field investigation (LFI) was conducted to assess the applicability of interim remedial measures (IRM) for reducing human health and environmental risks within the 100-BC-5 Groundwater Operable Unit. The 100-BC-5 Operable Unit is one of three operable units associated with the $100 \mathrm{~B} / \mathrm{C}$ Area. Operable units 1 and 2 address contaminant sources while 100-BC-5 addresses contamination present in the underlying groundwater. The primary method of investigation used during this LFI was the installation of monitoring wells. Samples were collected from the groundwater and soils submitted for laboratory analysis. Boreholes were surveyed for radiological contamination using downhole geophysical techniques to further delineate the locations and levels of contaminants. All samples were screened to ascertain the presence of volatile organic compounds and radionuclides. Analytical data were subjected to validation; all first round and $10 \%$ of the subsequent rounds of data associated with the LFI were validated. The screening method was used to identify contaminants of potential concern (COPC). This screening method eliminated from further consideration constituents that were below background. Constituents considered nontoxic to humans were eliminated from the human health evaluation. Inconsistency and blank contamination were also evaluated in the screening process. These COPCs were evaluated further in the qualitative risk assessment (QRA). Tritium and strontium-90 were identified as contaminants of concern at 100-BC-5 because the concentrations exceeded potential applicable or relevant and appropriate requirements. The QRA determined that the risk is low for all of the site contaminants.

161. U.S. Department of Energy. 1996. Biannual Recalibration of Two Spectral Gamma-Ray Logging Systems Used for Baseline Characterization Measurements in the Hanford Tank Farms. Vadose Zone Characterization Project at the Hanford Tank Farms. GJPO-HAN-3, U.S. Department of Energy, Grand Junction Office, Grand Junction, Colorado. ABSTRACT: The U.S. Department of Energy's (DOE's) Grand Junction Project Office (GJPO) is engaged in establishing an initial, or baseline, characterization of the gamma-ray-emitting contaminants in the subsurface of the Tank Farms at the DOE Hanford Site in Washington State. These baseline data are gathered by logging existing monitoring boreholes with two high-resolution passive gamma-ray logging systems informally known as Gamma 1 and Gamma 2. Calibration of the logging systems is crucial to the assurance of data quality. The project document Spectral Gamma-Ray borehole Geophysical Logging Characterization and Baseline Monitoring Plan for the Hanford Single-Shell Tanks (DOE 1995a) specifies that the initial, or base, calibration of both systems must be performed before commencement of field measurements at Hanford and that both systems must be recalibrated every 6 months, thereafter, using the calibration standards at the Hanford borehole logging calibration center. Data collection for the base calibrations was completed in April 1995; the results were published in Calibration of Two Spectral Gamma-Ray Logging Systems for Baseline Characterization Measurements in the Hanford Tank Farms (DOE 1995b). This report documents the first recalibration of the two systems that was performed in October 1995 at the Hanford Site. Analyses of data collected during the recalibrations are presented. 
162. U.S. Department of Energy. 1996. Vadose Zone Characterization Project at the Hanford Tank Farms, SX Tank Farm Report. DOE/ID/12584-268, GJPO-HAN-4, U.S. Department of Energy, Albuquerque Operations Office and Grand Junction Office for Richland Operations Office, Richland, Washington.

163. U.S. Department of Energy. 1997. Vadose Zone Characterization Project at the Hanford Tank Farms: BY Tank Farm Report. GJO-96-2-TAR, GJO-HAN-6, U.S. Department of Energy, Grand Junction Office, Grand Junction, Colorado. ABSTRACT: The U.S. Department of Energy Grand Junction Office (GJO) was tasked by the DOE Richland Operations Office (DOE$\mathrm{RL})$ to perform a baseline characterization of the contamination distributed in the vadose zone sediment beneath and around the single-shell tanks (SSTs) at the Hanford Site. The intent of this characterization is to determine the nature and extent of the contamination, identify contamination sources, and develop a baseline of the contamination distribution that will permit future data comparisons. This characterization work also allows an initial assessment of the impacts of the vadose zone contamination as required by the Resource Conservation and Recovery Act (RCRA). This characterization project involves acquiring information about the vadose zone contamination with borehole geophysical logging methods and documenting that information in a series of reports. Data from boreholes surrounding each tank are compiled into individual Tank Summary Data Reports. The data from each tank farm is then compiled and summarized in a Tank Farm Report. This document is the Tank Farm Report for the BY Tank Farm.

164. U.S. Department of Energy. 1997. Vadose Zone Characterization Project at the Hanford Tank Farms: U Tank Farm Report. GJO-97-1-TAR, GJO-HAN-8, U.S. Department of Energy, Grand Junction Office, Colorado. ABSTRACT: The U.S. Department of Energy Grand Junction Office (DOE-GJO) was tasked by the DOE Richland Operations Office (DOE-RL) to perform a baseline characterization of the gamma-ray-emitting radionuclides that are distributed in the vadose zone sediments beneath and around the single-shell tanks (SSTs) at the Hanford Site. The intent of this characterization is to determine the nature and extent of the contamination, identify contamination sources when possible, and develop a baseline of the contamination distribution that will permit future data comparisons. This characterization work also allows an initial assessment of the impacts of the vadose zone contamination as required by the Resource Conservation and Recovery Act (RCRA). This characterization project involves acquiring information regarding vadose zone contamination with borehole geophysical logging methods and documenting that information in a series of reports. This information is presently limited to detection of gamma-emitting radionuclides from both natural and man-made sources. Data from boreholes surrounding each tank are compiled into individual Tank Summary Data Reports. The data from each tank in a tank farm is then compiled and summarized in a Tank Farm Report. This document is the Tank Farm Report for the U Tank Farm. Logging operations used high-purity germanium detection systems to acquire laboratory-quality assays of the gamma-emitting radionuclides in the sediments around and below the tanks. These assays were acquired in 59 boreholes that surround the U Tank Farm tanks. 
165. U.S. Department of Energy. 1998. Hanford Tank Farms Vadose Zone, TX Tank Farm Report. GJO-97-13-TAR, GJO-HAN-11, U.S. Department of Energy, Albuquerque Operations Office and Grand Junction Office for Richland Operations Office, Richland, Washington.

ABSTRACT: Contains baseline spectral gamma log data for the 241-TX tank farm.

166. U.S. Department of Energy. 1998. Hanford Tank Farms Vadose Zone, AX Tank Farm Report. GJO-97-14-TAR, GJO-HAN-12, U.S. Department of Energy, Albuquerque Operations Office and Grand Junction Office for Richland Operations Office, Richland, Washington.

ABSTRACT: Contains baseline spectral gamma log data for the 241-AX tank farm.

167. U.S. Department of Energy. 1998. Hanford Tank Farms Vadose Zone, TY Tank Farm Report. GJO-97-30-TAR, GJO-HAN-16, U.S. Department of Energy, Albuquerque Operations Office and Grand Junction Office for Richland Operations Office, Richland, Washington.

ABSTRACT: Contains baseline spectral gamma log data for the 241-TY tank farm.

168. U.S. Department of Energy. 1998. Hanford Tank Farms Vadose Zone, S Tank Farm Report. GJO-97-31-TAR, GJO-HAN-17, U.S. Department of Energy, Albuquerque Operations Office and Grand Junction Office for Richland Operations Office, Richland, Washington.

ABSTRACT: Contains baseline spectral gamma log data for the 241-S tank farm.

169. U.S. Department of Energy. 1998. Hanford Tank Farms Vadose Zone, C Tank Farm Report. GJO-98-39-TAR, GJO-HAN-18, U.S. Department of Energy, Albuquerque Operations Office and Grand Junction Office for Richland Operations Office, Richland, Washington.

ABSTRACT: Contains baseline spectral gamma log data for the 241-C tank farm.

170. U.S. Department of Energy. 1998. Hanford Tank Farms Vadose Zone, BX Tank Farm Report. GJO-98-40-TAR, GJO-HAN-19, U.S. Department of Energy, Albuquerque Operations Office and Grand Junction Office for Richland Operations Office, Richland, Washington.

ABSTRACT: Contains baseline spectral gamma log data for the 241-BX tank farm.

171. U.S. Department of Energy. 1999. Vadose Zone Characterization Project at the Hanford Tank Farms, A Tank Farm Report. GJO-98-64-TAR, GJO-HAN-23, U.S. Department of Energy, Albuquerque Operations Office and Grand Junction Office for Richland Operations Office, Richland, Washington. ABSTRACT: Contains baseline spectral gamma log data for the 241-A tank farm.

172. U.S. Department of Energy. 1999. Vadose Zone Characterization Project at the Hanford Tank Farms, T Tank Farm Report. GJO-99-101-TAR, GJO-HAN-27, U.S. Department of Energy, Albuquerque Operations Office and Grand Junction Office for Richland Operations Office, Richland, Washington. ABSTRACT: Contains baseline spectral gamma log data for the 241-T tank farm. 
173. Van Luik, A. E., and R. M. Smith. 1982. 216-S-1 and S-2 Mixed Fission Product Crib Characterization Study. RHO-ST-39, Rockwell Hanford Operations, Richland, Washington. ABSTRACT: Borehole scintillation and gamma spectroscopy data are given in this report.

174. Waste Management Federal Services. 1998. Operational Environmental Monitoring. WMNW-CM-004, Waste Management Federal Services, Northwest Operations, Inc., Richland, Washington.

175. Webster, G. D., and J. W. Crosby. 1982. Stratigraphic Investigation of the Skagit/Hanford Nuclear Project, Sagit/Hanford Nuclear Project-Preliminary Safety Analysis Report, Vol. 6 and 6, Appendix 2R, Puget Sound Power and Light, Seattle, Washington.

176. Weekes, D. C., K. A. Lindsey, B. H. Ford, and G. K. Jaeger. 1996. Preoperational Baseline and Site Characterization Report for the Environmental Restoration Disposal Facility. Volume 2, Revision 2, BHI-00272, Bechtel Hanford, Inc., Richland, Washington. ABSTRACT: This document is Volume 2 in a two-volume series that comprises the site characterization report, the Preoperational Baseline and Site Characterization Report for the Environmental Restoration Disposal Facility. Volume 1 contains data interpretation and information supporting the conclusions in the main text. This document presents original data in support of Volume 1 of the report. The following types of data are presented: well construction reports; borehole logs; borehole geophysical data; well development and pump installation; survey reports; preoperational baseline chemical data, and aquifer test data. Five groundwater monitoring wells, six deep characterization boreholes, and two shallow characterization boreholes were drilled at the Environmental Restoration Disposal Facility (ERDF) site to directly investigate site-specific hydrogeologic conditions.

177. Westinghouse Hanford Company (WHC). 1996. Environmental Investigations and Site Characterization, Section 11.1, Geophysical Logging. WHC-CM-7-7, Westinghouse Hanford Company, Richland, Washington.

178. Westinghouse Hanford Company (WHC). Spectral Gamma-Ray Logging for the 100-N Area, Hanford, Washington. WHC-SD-EN-TI-261, Westinghouse Hanford Company, Richland, Washington. ABSTRACT: The objective of this effort was to delineate the vertical distribution and concentration of anthropogenic radionuclides in the subsurface surrounding nine boreholes in the 100-N Area available for geophysical logging with the Radionuclide Logging System (RLS). Cesium was defined in eight boreholes and the ninth hole was found to not contain any such radionuclides.

179. Williams, B. A. 1992. Borehole Completion DataPackage for DOE 216-S-10 Facility, CY 1991. WHC-SD-EN-DP-045, Westinghouse Hanford Company, Richland, Washington. ABSTRACT: This report contains gross gamma-ray logs for three new wells.

180. Williams, B. A., and. D. B. Barnett. 1993. Borehole Completion Data Package for the 216-S-10 Facility, CY-1992. WHC-SD-EN-DP-052, Westinghouse Hanford Company, Richland, Washington. 
181. Williams, B. A. 1994. Borehole Data Package for the 100-K Area Groundwater Wells, CY 1994. WHC-SD-EN-DP-090, Westinghouse Hanford Company, Richland, Washington.

ABSTRACT: Borehole, hydrogeologic and geophysical logs, drilling, as-built diagrams, sampling, and well construction information and data for RCRA-compliant groundwater monitoring wells installed in CY 1994 at the 100-K Basins.

182. IIPNLATLASIGeoDatalLg-Archv. ABSTRACT: This is the address of an unpublished library of all available spectral gamma-ray and neutron moisture logs acquired by the Radionuclide Logging system since the early 1990s. The library is maintained by Waste Management Federal Services, Northwest Operations on a Pacific Northwest National Laboratory file server.

183. IISACS \CASS. ABSTRACT: This is the address of an unpublished library of available gross gamma-ray logs acquired from single-shell tank farms.

184. http://www.doegjpo.com. ABSTRACT: This is the home page for the Department of Energy's Grand Junction Projects Office. All spectral gamma-ray logs acquired for single-shell tank farm characterization can be accessed through this internet site. 


\section{Distribution}

No. of

\section{Copies}

\section{OFFSITE}

Sunnie A. Aburime

Clark Atlanta University

Department of Engineering

223 James P. Brawley Dr.

P.O. Box 808

Atlanta, GA 30314

James N. Albright

Los Alamos National Laboratory

GeoEngineering Group

MSIN D443

Los Alamos, NM 87544

Patricia Ann Berge

Lawrence Livermore National Laboratory

7000 East Avenue, L-201

Livermore, CA 94550

David L. Bish

Los Alamos National Laboratory

MS D469

Los Alamos, NM 87545

James R. Brainard

Sandia National Laboratories

MS 0735

P.O. Box 5800

Albuquerque, NM 87185

Wesley L. Bratton

Applied Research Associates, Inc.

3250 Port of Benton Blvd.

Richland, WA 99352
No. of

\section{Copies}

Charles R. Carrigan

Lawrence Livermore National Laboratory

L-204

Flow \& Transport Group

P.O. Box 808

Livermore, CA 94550

Peter M. Castle

Idaho National Engineering and

Environmental Laboratory (BBWXTI)

P.O. Box 1625, MSIN 2208

Idaho Falls, ID 83415

William P. Clement

Boise State University / CGISS

MG206

1910 University Drive

Boise, ID 83725-1536

Michael P. Connelly

HydroGeologic, Inc.

3250 Port of Benton Blvd.

Richland, WA 99352

William D. Daily

Lawrence Livermore National Laboratory

L-130

Livermore, CA 94550

Donald J. DePaolo

Lawrence Berkeley National Laboratory

Department of Earth and Planetary Sciences

MS 4767

1 Cyclotron Road

Berkeley, CA 94720-4767 
No. of

Copies

Dirk Dunning

Oregon Office of Energy

625 Mariona St. N.E.

Salem, OR 97301-3742

Eric Dysland

WPI

3250 Port of Benton Blvd.

Richland, WA 99352

Boris Faybishenko

Lawrence Berkeley National Laboratory

Earth Sciences Division

One Cyclotron Road

MS 90-1116

Berkeley, CA 94720

James B. Fink

HydroGEOPHYSICS, Inc.

5865 South Old Spanish Trail

Tucson, AZ 85747

Judit German-Heins

Nez Perce Tribe, ERWM

P.O. Box 365

Lapwai, ID 83450

Masoud Ghodrati

University of California-Berkeley

Ecosystem Sciences Division

151 Hilgard Hall

Berkeley, CA 94720-3110

Molly M. Gribb

University of South Carolina

Department of Civil and Environmental

Engineering

300 Main Street

Columbia SC 29208
No. of

Copies

Michael Hoversten

Lawrence Berkeley National Laboratory

One Cyclotron Road

MSIN 90-1116

Berkeley, CA 94720

James D. Irving

University of British Columbia

129 - 2219 Main Mall

Vancouver, BC

Canada V6T1Z4

Rosemary J. Knight

University of British Columbia

2219 Main Mall

Vancouver, BC

Canada V6T 1 Z4

B. Sue Lantz

U.S. Department of Energy-ID

850 Energy Drive

EMSP - MS 1225

Idaho Falls, ID 83401

Richard E. Lewis

Schlumberger HydroGeological

Technologies

6090 Greenwood Plaza Blvd.

Englewood, CO 80111

Peter C. Lichter

Los Alamos National Laboratory

Environmental Sciences Division (EES-5)

MS F-649

Los Alamos, NM 87545

Distr.2 
No. of

Copies

Stephen H. Lieberman

Space and Naval Warfare Systems Center,

San Diego

Environmental Sciences Division, D361

53475 Strothe Road

San Diego, CA 92152

Kevin A. Lindsey

Daniel B. Stephens \& Associates, Inc.

Geoscientists, Hydrologists, and Engineers

719 Jadwin Ave., \#28

Richland, WA 99352

Ernest L. Majer

Lawrence Berkeley National Laboratory

1 Cyclotron Road (MS 90-116

Berkeley, CA 94720

Earl D. Mattson

Idaho National Engineering and

Environmental Laboratory

P.O. Box 1625

2251 N. Boulevard

Idaho Falls, ID 83415-2107

Stephen M. J. Moysey

University of British Columbia

6339 Stores Road

Vancouver, British Columbia

Canada V6T 1Z4

Dolly A. McElroy

WPI

3250 Port of Benton Blvd.

Richland, WA 99352

Bruce McNamara

U.S. Department of Energy-HQ

Richland Operations

19901 Germantown Road

Germantown, MD 20874-1290
No. of

Copies

Brent D. Newman

Los Alamos National Laboratory

Environmental Science Group

MS J495, LANL

Los Alamos, NM 87545

Gregory A. Newman

Sandia National Laboratories

P.O. Box 5800

MS-0750

Albuquerque, NM 87185-0750

Karsten Pruess

Lawrence Berkeley National Laboratory

Sr. Scientist, Earth Sciences Division,

MS 90-1116

One Cyclotron Road

Berkeley, CA 94720

Wade Riggsbee

Yakima Nation

1933 Jadwin

Richland, WA 99352

Michael Ritzwoller

University of Colorado

Campus Box 390

2000 Colorado Ave.

Duane Physics Building, Rm. F725

Boulder, CO 80309

Garland C. Roberston

Alabama A\&M University

P.O. Box 1208

Nomal, AL 35762

John Seaman

Savannah River Ecology Lab

Drawer E

Aiken, SC 29802 
No. of

Copies

James B. Sisson

Idaho National Engineering and

Environmental Laboratory, BBWI

P.O. Box 1625, MS-2107

Idaho Falls, ID 83415-2107

Everett P. Springer

Los Alamos National Laboratory

Environmental Science Group

MSJ495

Los Alamos, NM 87545

Carl I. Steefel

Lawrence Livermore National Laboratory

L-204

P.O. Box 808

Livermore, CA 94551

John Ullo

Schlumberger-Doll Research

Old Quarry Road

Ridgefield, CT 06877

Peter Weichman

Blackhawk Geometrics

301 Commercial Road, Suite B

Golden, CO 80401

John L. Wilson

New Mexico Institute of Mining and

Technology

Department of Earth \& Environmental

Science

New Mexico Institute of Mining

Socorro, NM 87801

Ivana Witt

Yakima Nation

1933 Jadwin

Richland, WA 99352
No. of

Copies

T.C. Jim Yeh

University of Arizona

Department of Hydrology and Water

Resources

The University of Arizona, Bldg. 11

Tucson, AZ 85721

Dongxiao Zhang

Los Alamos National Laboratory

Environmental Science Group

MS C306

Los Alamos, NM 87545

\section{ONSITE}

\section{DOE Office of River Protection}

CA Babel

H6-60

RM Yasek

H6-60

6 DOE Richland Operations Office

BL Foley

P7-62

JP Hanson

K8-50

RD Hildebrand

A5-13

DE Olson

A5-13

D Tano

A7-75

KM Thompson

A5-13

8 Bechtel Hanford Inc.

RL Biggerstaff

$\mathrm{H} 0-02$

AG Dada

$\mathrm{H} 0-02$

KR Fecht

$\mathrm{H} 0-02$

BH Ford

H0-19

MJ Graham

H0-09

GB Mitchem

H0-21

SW Petersen

$\mathrm{H} 0-02$

TM Wintzcak

H0-21 
No. of

Copies

10 CH2M Hill Hanford Group

KA Bergstrom (2)

AJ Knepp

WJ McMahon

TH Mitchell (2)

VJ Rohay (2)

LC Swanson

CD Wittreich

3 Flour Federal Services.,

EJ Freeman

B4-43

R Khaleel

B4-43

FM Mann

IT Corporation

DA Myers

H0-22

2 MACTER-ERS

JF Bertsch

B1-42

RG McCain

H9-02

$\mathrm{H} 0-22$

H9-03

H9-02

H0-19

H9-02

H9-03

H-022

B1-42

3 Waste Management Technical Services

MG Gardner

H1-11

JE Meisner

H1-11

RK Price

H1-11

\section{Pacific Northwest National Laboratory}

WF Bonner

A Chilakapati
K9-14

K9-36
No. of

Copies

MJ Fayer

MD Freshley

K9-33

H0-21

K6-81

K9-33

K6-81

K6-81

K6-96

K6-81

K2-31

K9-33

K9-33

K3-54

H0-21

BPO

K8-03

K6-81

K6-96

K9-33

K6-81

K6-81

K5-25

K6-81

K6-81

K6-81

K2-10

K9-33

K9-36

K6-81

K9-36

K9-36

H0-21

K8-12

K8-96

K1-06 